\title{
Irritable bowel syndrome : pathophysiology, symptoms and biomarkers
}

Citation for published version (APA):

Mujagic, Z. (2015). Irritable bowel syndrome : pathophysiology, symptoms and biomarkers. [Doctoral Thesis, Maastricht University]. Uitgeverij BOXPress || Proefschriftmaken.nl.

https://doi.org/10.26481/dis.20151221zm

Document status and date:

Published: 01/01/2015

DOI:

10.26481/dis.20151221zm

Document Version:

Publisher's PDF, also known as Version of record

\section{Please check the document version of this publication:}

- A submitted manuscript is the version of the article upon submission and before peer-review. There can be important differences between the submitted version and the official published version of record.

People interested in the research are advised to contact the author for the final version of the publication, or visit the DOI to the publisher's website.

- The final author version and the galley proof are versions of the publication after peer review.

- The final published version features the final layout of the paper including the volume, issue and page numbers.

Link to publication

\footnotetext{
General rights rights.

- You may freely distribute the URL identifying the publication in the public portal. please follow below link for the End User Agreement:

www.umlib.nl/taverne-license

Take down policy

If you believe that this document breaches copyright please contact us at:

repository@maastrichtuniversity.nl

providing details and we will investigate your claim.
}

Copyright and moral rights for the publications made accessible in the public portal are retained by the authors and/or other copyright owners and it is a condition of accessing publications that users recognise and abide by the legal requirements associated with these

- Users may download and print one copy of any publication from the public portal for the purpose of private study or research.

- You may not further distribute the material or use it for any profit-making activity or commercial gain

If the publication is distributed under the terms of Article $25 \mathrm{fa}$ of the Dutch Copyright Act, indicated by the "Taverne" license above, 


\section{IRRITABLE BOWEL SYNDROME}

Pathophysiology, Symptoms and Biomarkers 
${ }^{0}$ Copyright Zlatan Mujagić, Maastricht 2015

Layout: Tiny Wouters

Cover: Stefani Wiatowski

Printed by: Uitgeverij BOXPress || Proefschriftmaken.nl

ISBN: 978-94-6295-402-1

The studies presented in this thesis were performed within the framework of NUTRIM School of Nutrition and Translational Research in Metabolism (Maastricht University), which participates in the Graduate School VLAG (Food Technology, Agrobiotechnology, Nutrition and Health Sciences), accredited by the Royal Netherlands Academy of Arts and Sciences. Furthermore, a part of the research presented in this thesis has been funded by the Top Institute for Food and Nutrition (TIFN), Wageningen.

Printing of this thesis was financially supported by: Grünenthal GmbH, Aachen, Germany - Medische Laboratoria Dr. Stein \& Collegae, Maastricht, The Netherlands - Euro Diagnostica, Malmö, Sweden Nederlandse Vereniging voor Gastroenterologie (NVGE) - Maastricht University Medical Center+. 


\title{
IRRITABLE BOWEL SYNDROME
}

\section{Pathophysiology, Symptoms and Biomarkers}

\begin{abstract}
ACADEMISCH PROEFSCHRIFT
ter verkrijging van de graad van doctor aan de Universiteit Maastricht, op gezag van de Rector Magnificus, Prof. dr. L.L.G. Soete volgens het besluit van het College van Decanen, in het openbaar te verdedigen op maandag 21 december 2015, om 12:00 uur.
\end{abstract}

door

Zlatan Mujagić 
Promotor

Prof. dr. A.A.M. Masclee

Prof. dr. F.J. van Schooten

\section{Copromotor}

Dr. D.M.A.E. Jonkers

\section{Beoordelingscommissie}

Prof. dr. C.H.C. Dejong; voorzitter

Prof. dr. A.M. Schols

Prof. dr. R.C. Spiller, Queen's Medical Centre, Nottingham, UK

Prof. dr. H.W.M. Steinbusch

Dr. P.J.J. van der Veek, Medisch Centrum Haaglanden, Den Haag 


\section{Table of contents}

$\begin{array}{lll}\text { Chapter } 1 & \text { Introduction } & 7\end{array}$

$\begin{array}{lll}\text { Part I } & \text { Pathophysiology } & 23\end{array}$

Chapter 2 Intestinal permeability in IBS 25

Chapter $3 \quad$ Effects of L. plantarum on intestinal mucosa

Chapter $4 \quad$ Effects of L. plantarum on immunity 71

Chapter 5 Markers for visceral hypersensitivity in IBS 99

Chapter $6 \quad$ Biological markers for visceral hypersensitivity in IBS 115

$\begin{array}{lll}\text { Chapter } 7 & \text { Serotonin metabolism in IBS } & 131\end{array}$

$\begin{array}{lll}\text { Part II } & \text { Symptoms } & 153\end{array}$

Chapter 8 Systematic review: Measurement of abdominal pain in IBS 155

Chapter $9 \quad$ ESM - a new digital tool for symptom assessment in IBS 189

$\begin{array}{lll}\text { Part III } & \text { Biomarkers } & 207\end{array}$

Chapter 10 A novel faecal and plasma biomarker panel for IBS 209

Chapter 11 Novel breath biomarkers for IBS; a metabolomic approach 229

$\begin{array}{lll}\text { Chapter } 12 \text { General discussion } & 247\end{array}$

$\begin{array}{ll}\text { Summary } & 261\end{array}$

$\begin{array}{ll}\text { Samenvatting } & 269\end{array}$

$\begin{array}{ll}\text { Valorisation } & 277\end{array}$

$\begin{array}{ll}\text { List of publications } & 285\end{array}$

$\begin{array}{ll}\text { Dankwoord } & 291\end{array}$

$\begin{array}{ll}\text { Curriculum vitae } & 301\end{array}$ 



\section{Chapter 1}

General introduction 
Chapter 1 


\section{General introduction}

\section{Functional Gastrointestinal Disorders}

Functional gastrointestinal disorder (FGID) is an umbrella term for a broad spectrum of disorders of the gastrointestinal (GI) tract, for which no clearcut organic cause can be identified. ${ }^{1,2}$ FGIDs are among the most prevalent disorders worldwide. Since only a minority of people who experience functional GI symptoms seek medical attention, ${ }^{3}$ the exact prevalence of FGIDs among the general population is not well established. In a large general population based prospective cohort study in the USA, Halder et al. found that $42 \%$ of the included subjects had one or more FGIDs, as observed over a 12 year time period. ${ }^{4}$

The first FGID to be described in 1818, was a disorder characterized by abdominal pain, now referred to as irritable bowel syndrome (IBS). ${ }^{5}$ In the past decades, several terms have been used for IBS, mostly based on altered motility and sensitivity of the colon as the presumed main pathophysiological mechanism, such as spastic colon, nervous colon or irritable colon syndrome. ${ }^{6}$ The term IBS was first introduced in 1967 by DeLor. ${ }^{7}$ In 1978, Manning and colleagues described the first criteria based on symptoms to aid the diagnostic process for IBS (i.e. the Manning criteria). ${ }^{8}$ In 1989, the Rome Committee published new criteria for $\mathrm{IBS},{ }^{9}$ which were revised several times, resulting in the currently generally accepted 'gold standard' Rome III criteria. Meanwhile, discussion is ongoing, which should lead to the development of the Rome IV criteria in the nearby future. ${ }^{10}$

\section{Irritable Bowel Syndrome}

The diagnosis of IBS, as described by the Rome III criteria, is based on recurrent abdominal pain or discomfort, which patients experience at least 3 days per month, and is associated with changes in stool frequency, consistency, and/or improvement with defecation. Furthermore, symptom onset must be at least 6 months before making the diagnosis. Furthermore, 'red flags' that could indicate the presence of an organic disorder, should be absent. Based on a meta-analysis, a pooled estimate of $11 \%$ has been reported for the global prevalence of IBS among the general population, with a female predominance $\left(1: 2\right.$ ratio). ${ }^{11}$

Next to abdominal pain or discomfort, several other Gl symptoms, such as bloating, flatulence, constipation and/or diarrhoea, nausea and belching, have been associated with IBS, but their presence and severity vary between patients. IBS can be divided into four subtypes according to predominant bowel habits, i.e. diarrhoea (IBS-D) and constipation (IBS-C) predominant and mixed (IBS-M) subtypes, or those with unspecified stool pattern (IBS-U). ${ }^{1}$ Furthermore, the IBS patient population is characterised by a large diversity in lifestyle, dietary patterns and use of medication. Altogether, this reflects the distinct heterogeneity of the IBS patient population. 
From a clinical perspective, IBS is not considered a severe medical condition, nor is it a risk factor or predisposing to other more severe disorders. Nevertheless, the disease burden in affected subjects is high, leading to considerable impairment of quality of life and work absenteeism, ${ }^{12}$ and high health care costs. ${ }^{13}$ High costs are reflected by extensive diagnostic investigations, as well as the lack of standardised treatment protocols. ${ }^{13}$ Although the Rome III criteria emphasize that IBS is not a diagnosis of exclusion and should be based on symptom criteria, ${ }^{1}$ in daily clinical practice most physicians (>70\%) frequently employ endoscopy, CT or ultrasound imaging, blood, faecal or other analyses to exclude organic disorders. ${ }^{14}$ Objective non-invasive biomarkers that could aid the diagnostic process of IBS in daily clinical practice are currently not available. ${ }^{15}$ Furthermore, standardized treatments for IBS are lacking. The efficacy of currently available therapeutic strategies is moderate. ${ }^{16}$ This is partly due to a lack of insight into pathophysiological mechanisms that underlie generation of $\mathrm{GI}$ symptoms in IBS.

\section{Pathophysiology of IBS}

Although IBS is a symptom-based diagnosis without a clear organic cause, several pathophysiological mechanisms have been associated with the disorder. This has led to the generally accepted concept of multifactorial aetiology in IBS. Alterations may occur in many intestinal processes, in bidirectional signalling between the central nervous system and the $\mathrm{Gl}$ tract, in processing of signals by the brain, in prevalent psychological comorbidity and in genetic susceptibility. Through complex interaction all these factors contribute to the development of IBS, as is shown in Figure 1.1. ${ }^{16,17}$

\section{Genetics}

A role for host genetics in IBS originates from findings in family studies. IBS patients were reported to be twice more likely to have relatives with IBS compared to non-IBS subjects. ${ }^{18}$ However, this association may also result from shared environmental and social factors instead of genetic aspects. Remarkably, a significantly greater concordance for IBS has been reported in monozygotic compared to dizygotic twins. ${ }^{19}$

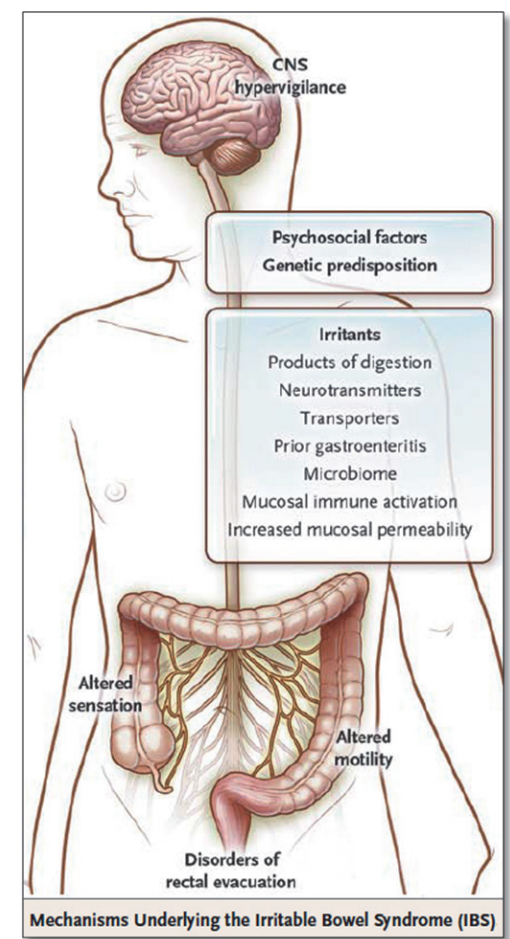

Figure 1.1 Reproduced with permission from (Camilleri M., N Engl J Med, 2012) Copyright Massachusetts Medical Society. 
Several genetic variations have been associated with the presence of IBS, such as polymorphisms of the gene that encodes for the serotonin transporter (SERT) or for the cytokine TNF superfamily member 15 (TNFSF15). ${ }^{20,21}$ Up to now, there is little evidence for genetics as a major factor in the pathophysiology of IBS. ${ }^{22}$ It is more likely that symptoms arise as a consequence of gene-environment interactions, which indicates that triggers during life are needed in subjects with a specific genetic background, in order to develop IBS.

\section{The immune system, intestinal barrier function and microbiota}

An acute GI infection is a well-known trigger for the development of IBS in a subgroup of patients, defined as post infectious (PI) IBS. This subgroup, PI-IBS, has been described for the first time in the 1950s. ${ }^{23}$ In 2000 in Walkerton Ontario, a large waterborne outbreak of bacterial dysentery (based on Escherichia coli, Campylobacter jejuni and other pathogens) provided a unique opportunity to study the long-term effects of acute GI infections. In the Walkerton cohort including 2069 subjects, 36\% did develop IBS, versus $10 \%$ of controls without a previous gastroenteritis. ${ }^{24}$ These findings support involvement of immune activation and the intestinal microbiota in the development of IBS.

At present there is accumulating evidence for a low-grade inflammatory state in IBS. The differences between IBS patients and healthy subjects observed in most studies are only subtle, e.g. with regard to increased faecal calprotectin concentrations, ${ }^{25}$ increased mucosal infiltration of immune cells or with regard to an imbalance in plasma cytokine profiles in IBS patients. ${ }^{26-29}$ Other studies have focused more on the relation between mucosal mast cell counts, their secretory activity (such as tryptase) and IBS symptoms. For example, Barbara et al. found an increased area of colon mucosal biopsies to be occupied by mast cells in $77 \%$ of the IBS patients compared with only $9 \%$ of controls. Furthermore, mucosal histamine and tryptase levels were significantly increased, and mast cells were more often located in close proximity to nerve endings in IBS patients compared to healthy subjects. The latter finding correlated significantly with the severity of abdominal pain/discomfort. ${ }^{30}$ More recently, Vanuytstel et al. demonstrated that acute psychological stress increased small intestinal permeability in healthy subjects. Administration of corticotropin-releasing hormone (CRH) was found to induce similar effects that could be inhibited by the mast cell stabiliser disodium cromoglycate (DSCG). ${ }^{31}$ These findings provide exciting new insights into interactions between brain, mast cell activity and intestinal barrier function.

A single epithelial layer of the intestinal mucosa facilitates permeation and transport of nutrients and other substances through selective transcellular and paracellular routes and forms an important part of the intestinal barrier. The paracellular passage is regulated by complexes of tight junctions, adherens junctions, gap junctions and desmosomes. Dysfunction of these complexes leads to impairment of the intestinal 
barrier function and increased intestinal permeability. ${ }^{32}$ Several studies have reported on increased intestinal permeability in IBS patients compared to healthy controls. This seemed most pronounced for $\mathrm{PI}-I B S^{33-35}$ or IBS-D patients. ${ }^{36-38}$ it should be acknowledged that these data were based on only small study populations. Other investigators have not been able to confirm the findings of increased permeability in IBS. $^{39,40}$ The presumed barrier dysfunction in (subgroups of) IBS patients may result from immune activation, from altered intestinal microbiota composition, and physiological stress, but also from environmental factors, such as alcohol and use of non-steroid anti-inflammatory drugs (NSAIDs). Furthermore, increased permeation of bacteria and their products (e.g. lipopolysaccharides) may also lead to local and systemic inflammatory responses, ${ }^{41}$ resulting in a vicious circle. Further research is needed to investigate if and how all these factors interact and contribute to IBS and its subtypes.

Alterations in microbiota composition and metabolic activity could be an important link in the interplay between $\mathrm{Gl}$ infections as a trigger in a subgroup of patients, low-grade inflammation and barrier dysfunction in IBS. Marked alterations in the composition of faecal microbiota and a reduction in diversity of the faecal microbiota have been reported in IBS compared to controls. ${ }^{42-44}$ However, overall findings with respect to specific bacterial groups or species are not consistent and data on the association of microbiota composition and IBS subtypes or other patient characteristics are limited. The intestinal microbiome interacts with exogenous (e.g. metabolisation of dietary compounds) and endogenous factors (e.g. modulation of epithelial integrity and immune responses). ${ }^{45}$ Thereby not only the composition but also the functional capacity of the microbiome may affect pathophysiological mechanisms and symptom development in IBS.

A recent meta-analysis showed that administration of potentially beneficial living bacteria (i.e. probiotics) has a (moderate) beneficial effect on overall GI symptoms in patients with IBS. ${ }^{46}$ This has been attributed to their potential effects on the microbiota composition and activity, as well as for example gut barrier function and immune activation. However, these findings regarding the interplay between microbiota and the host were mainly based on in vitro studies and on a limited number of in vivo data in healthy subjects. ${ }^{47-52}$ Further research is needed to explore the exact mechanisms by which orally consumed living bacteria interact with the intestinal mucosa in humans, and how they may contribute to intestinal health under stressed conditions (for instance: after intake of NSAIDs) or in subjects with GI complaints or diseases.

Visceral hypersensitivity, gut-brain interaction, central nervous system modulation and psychological factors

Abdominal pain is a prominent symptom in patients with IBS and is considered to be primarily of visceral origin. Accordingly, increased visceral perception is found to be an 
important hallmark of IBS. ${ }^{53}$ In 1973 , Ritchie was the first to report that $55 \%$ of IBS patients versus $6 \%$ of healthy subjects experienced abdominal pain during inflation of a balloon introduced in the rectosigmoid. ${ }^{54}$ The electronic rectal barostat with a balloon of non-compliant material has become a widely accepted method to assess visceral hypersensitivity, but is also used to test the efficacy of therapeutic interventions. ${ }^{55,56}$ Later studies have confirmed Ritchie's original observation, demonstrating that around $50 \%$ of the IBS population can be classified with visceral hypersensitivity. ${ }^{53,57}$

Several studies investigated underlying mechanisms of altered visceral perception, but the exact pathophysiology is not yet clear. In 2009, Zhou et al. found an association between increased intestinal permeability and visceral hypersensitivity in IBS-D patients, $^{37}$ but this observation has not been replicated by others. The transient receptor potential vanilloid type-1 (TRPV1) has been shown to play a role in inflammatory hyperalgesia. ${ }^{58}$ It is expressed by sensory neurons and activated by capsaicin, heat, acid and inflammatory mediators. ${ }^{58,59}$ Akbar et al. demonstrated a threefold increase in the number of TRPV1 sensory nerve fibres in colonic biopsies of IBS patients compared to healthy controls; while no significant differences were seen between IBS-subtypes. Furthermore, CD3 immune cell and mast cell counts were higher in IBS patients compared to controls, and both the numbers of TRPV1 fibres and mast cells were found to be predictors of abdominal pain. ${ }^{60}$ Although, associations between indicators of intestinal barrier dysfunction, low-grade inflammation and altered visceral perception in IBS patients have been reported, the exact role of these biological processes in the aetiology of IBS, the development of visceral hypersensitivity and the mode of interaction is still not clear or understood. ${ }^{61}$

In addition to intestinal factors, mechanisms in central pain processing may contribute to abdominal pain in IBS patients. Central stimuli such as stress, affective co-morbidity, distraction or relaxation, and expectations have been found to alter visceral perception. $^{62-64}$ Functional magnetic resonance imaging (fMRI) studies have documented changes in the thalamus, prefrontal cortex, and the ventro-lateral and posterior parietal cortex in IBS patients, pointing to alterations in brain circuits involved in attention, emotion and pain modulation. ${ }^{64,65}$ Psychiatric co-morbidity is found to be highly prevalent in IBS patients. Several studies have demonstrated that up to $50 \%$ of IBS patients have a co-morbid anxiety disorder or depression. ${ }^{66-68}$ Hyper-reactivity to psychological stress is assumed to play an important role in symptom generation of IBS. The behavioural, neuroendocrine and autonomic responses to stress are co-regulated by the hypothalamic-pituitary-adrenal (HPA) axis and the sympathetic nervous system. Alterations in activity of brain and gut response to $\mathrm{CRH}$, adrenocorticotropin hormone (ACTH), cortisol, and catecholamines have been reported in IBS patients. ${ }^{29,31,69,70}$ Furthermore, altered serotonin metabolism is associated with IBS as well as with psychiatric disorders such as anxiety or depression. Serotonin is a neurotransmitter, with important functions in the central nervous system, such as regulation of mood, sleep and satiety. It is derived from tryptophan, and is primarily produced by 
enterochromaffin cells within the GI tract, where serotonin affects motoric, secretory and sensory functions of the intestines. ${ }^{71}$ Furthermore, $\mathrm{CRH}$ and serotonin have also been reported to affect gut barrier function.

In these studies, mechanisms associated with increased visceral perception have been explored, but since many pathophysiological mechanisms are associated with IBS in general, it remains unclear why some IBS patients can be classified as viscerally normosensitive, comparable to healthy subjects, while other IBS patients have increased pain perception.

Although, multiple pathophysiological mechanisms have been associated with the presence of IBS, the complex interplay between these processes and their association with symptom generation and subtypes of IBS is still poorly understood. Referring to the heterogeneity and the unclear aetiology of symptoms in IBS patients, William Cumming stated in 1849 that "The bowels are at one time constipated, another lax, in the same person. How the disease has two such different symptoms I do not profess to explain." ${ }^{72}$ Today, more than one and a half century later, further studies are still needed to elucidate this intriguing phenomenon.

\section{Symptom assessment in IBS}

IBS is based on a diagnosis related to presence of specific symptoms. Reduction of symptom severity is an important therapeutic goal in IBS patients. Therefore, symptom scores are often used as primary outcome of IBS intervention trials. However, investigating potential beneficial effects of various therapeutic strategies is challenging due to the subjective nature of symptom scoring and high placebo effect in IBS patients. $^{73}$ Due to a wide variety of methods that are employed for symptom assessment, the results of various studies are difficult to compare. To tackle this problem, in 2012, the US Food and Drugs Administrating (FDA) published guidelines for intervention trials in IBS, recommending a 7-day mean of an 'end-of-day 11-point numeric rating scale (NRS)' as a primary endpoint for clinical trials. ${ }^{74}$ Although several scales for the assessment of abdominal pain and other GI symptoms have been tested psychometrically in patients with $\mathrm{IBS}^{75-77}$ validation studies on the 11-point NRS recommended by the FDA are not available.

In general, assessment of symptoms poses several hurdles. The experience of $\mathrm{Gl}$ symptoms, in particular pain, can be altered by factors such as anxiety, depression, negative cognitions and positive or negative expectations. ${ }^{62-64}$ Taking into account the high prevalence of psychiatric co-morbidity in IBS, the presence of psychological factors is likely to affect symptom reporting in a heterogeneous group of subjects, as is the case in IBS. Not only perception of symptoms, but also memory can be affected by several psychological and circumstantial factors and may induce recall bias in methods that employ retrospective symptom assessment. Finally, it has been demonstrated that there is a high rate of fake compliance when filling in end-of-day diaries. Stone et al. 
found that only $10 \%$ of paper end-of-day diaries was completed during the indicated time frame in a population of subjects suffering from different forms of pain. ${ }^{78}$ Therefore, taking into account the cardinal importance of symptom assessment in IBS patients, new and more reliable symptom assessment methods need to be developed and validated for use in the target population.

\section{Biomarkers in IBS}

Although IBS is a functional GI disorder, accumulating evidence exists for subtle organic changes in a range of biological processes, possibly involved in the aetiology of IBS. There is ongoing interest in developing biomarkers based on these potential pathophysiological mechanisms. Such markers could aid the diagnostic process and may be used for the assessment of treatment efficacy and disease follow-up in IBS. ${ }^{15}$ As functional GI symptoms are very prevalent in the general population, an IBS biomarker may also be useful for the implementation in studies focusing on gut health in the general population.

An ideal biomarker should be able to discriminate between health and disease, and between the disease and other pathological conditions, should be sensitive for change in (patho)physiological state of the target population, and furthermore, should be reliable. $^{79}$ Multiple markers have been investigated as potential biomarker for IBS. These include intestinal permeability, visceral hypersensitivity, assessment of gut motility and transit time, genetic markers, faecal bile acids, and markers of inflammation or intestinal neuroendocrine activity in mucosal, faecal or blood samples. ${ }^{15}$ Most studies investigated single biomarkers, while two recent studies have tested a combination (panel) of 10 and 34 serum and genetic markers. ${ }^{80,81}$ However, up to now the use of biomarkers in IBS has led to only moderate results and none of the tested biomarkers or panels of markers have been accepted for the use in scientific trials or daily clinical practice. Taking into account that IBS is based on a multifactorial pathophysiology, a potential biomarker for IBS should be able to measure changes in several processes involved. Therefore, new approaches in the search for biomarkers for IBS should focus on marker panels; i.e. by combining 'known' biomarkers representing separate pathophysiological processes associated with IBS or by applying a new holistic '-omics' approach. For the latter, a wide range of compounds (such as proteins or volatile organic metabolites) can be measured in biomaterials and used to identify a profile of compounds with the best positive prediction for IBS. 


\section{Outline and aims}

IBS is a common functional GI disorder with major impact on quality of life and health care costs. Further characterisation of the patients and insight in underlying pathophysiological mechanisms, paying attention to the heterogeneity of IBS with regard to predominant symptoms and patient characteristics, may aid to elucidate the aetiology of IBS and to provide leads for new therapeutic options. Furthermore, accurate diagnosis and symptom assessment in IBS will facilitate future research and patient care in daily clinical practice. Therefore, in the current thesis, the pathophysiology, symptom assessment and biomarkers of IBS were addressed.

In Chapter 2 we focus on potential changes in intestinal permeability in IBS. There was need for a large scale study, investigating the association between altered intestinal permeability as an indicator of intestinal barrier dysfunction, with specific attention for (i) IBS-subtype differences, (ii) the GI site of potential altered permeability and (iii) the effects of potential confounding factors, such as the use of specific medication.

In Chapter 3 we investigate whether intestinal barrier (dys)function can be modulated by probiotic bacteria. Therefore, on separate occasions, the effect of three orally administrated Lactobacillus plantarum strains on small intestinal barrier function was investigated in healthy subjects after intake of the NSAID indomethacin, a drug known to induce intestinal barrier dysfunction. In addition to measurement of intestinal permeability, we collect duodenal biopsies for analysis of tight junction associated proteins and genome wide transcription analysis. In Chapter 4 the same study population was used to assess if and how these living bacteria affect the human intestinal and systemic immune system.

In Chapter 5 we focus on another pathophysiological process and hallmark of IBS, i.e. altered visceral perception. Most studies assessing this phenomenon report that about $50 \%$ of IBS patients can be classified as visceral hypersensitive. It is not clear to what extent hypersensitive and normosensitive IBS patients differ in clinical presentation, symptoms and demographics. Therefore, we assessed visceral perception in a large group of well-characterized IBS patients using a standardized rectal barostat procedure. Normo- and hypersensitive IBS patients were then compared with regard to demographic characteristics, lifestyle factors, psychological symptom scores and use of medication. In Chapter 6 we use the same approach to assess whether differences exist in biomarker data between hypersensitive and normosensitive IBS patients. The markers that have been selected relate to several pathophysiological mechanisms of IBS that may play a role in the development of visceral hypersensitivity, such as intestinal immune activation, altered activity of the neuroendocrine system and serotonin metabolism, and intraluminal metabolic processing by measuring various short chain fatty acids as products of saccharolytic fermentation by the intestinal microbiota. 
Finally, in Chapter 7 we focus further on the serotonin metabolism in IBS patients. Previous studies have reported altered plasma serotonin levels in IBS patients compared to healthy subjects. However, these findings were based on small study populations or only specific IBS subtypes. We assessed plasma concentrations of serotonin and its main metabolite 5-hydroxyindoleacetic acid (5-HIAA) in a large group of IBS patients and healthy controls, with specific attention for possible differences between IBS subtypes and increased scores of anxiety and depression.

As chronic abdominal pain is a prominent symptom in IBS patients and is often used as primary outcome in intervention trials, we focus on methods for symptom assessment, in particular abdominal pain, in the second part of this thesis. First, we systematically review the literature on currently available instruments to measure chronic abdominal pain in patients with IBS (Chapter 8). In psychological and behavioural research momentary symptom assessment by an electronic device is used to overcome limitations of retrospective symptom assessment using paper questionnaires or diaries, such as recall bias or low compliance rates. In Chapter 9 we explore possible advantages of Experience Sampling Method (ESM) over prevalently used retrospective paper symptom scores in a small group of IBS patients.

Reliable biomarkers that can be used next to symptom scores to aid the diagnosis and follow-up of IBS are currently not available. The third part of this thesis deals with the search for (sets of) biomarkers in IBS. We applied two different approaches using a study population of two large cohorts, namely a clinical IBS cohort with healthy controls without GI complaints, and a general population cohort. In Chapter 10 we test a set of 15 faecal and plasma markers, which were selected based on literature search as indicators of several domains of gut health, in both cohorts. The aim is to identify and validate a panel of markers which best discriminates IBS patients from healthy controls. Furthermore, we test the correlation between the biomarker panel and GI symptoms in IBS patients and subjects of the general population. Finally, we use the same cohorts to measure all detectable volatile organic compounds (VOCs) in exhaled breath, in search for a novel biomarker panel for IBS based on exhaled air (Chapter 11). Also in this case, the relation of the VOC biomarkers and GI symptoms in both cohorts has been investigated.

Finally, in Chapter 12 we summarize the main findings of the studies included in this thesis, and provide an integrated review of new insights obtained by these studies. 


\section{References}

1. Drossman DA, Corazziari E, Delvaux M, Spiller RC, Talley NJ, Thompson WG, Whitehead WE. Rome III: The Functional Gastrointestinal Disorders, Third Edition: McLean, VA: Degnon Associates, Inc.; 2006.

2. Longstreth GF, Thompson WG, Chey WD, Houghton LA, Mearin F, Spiller RC. Functional bowel disorders. Gastroenterology. 2006;130:1480-91.

3. Jones R, Lydeard S. Irritable bowel syndrome in the general population. BMJ. 1992;304:87-90.

4. Halder SL, Locke GR, 3rd, Schleck CD, Zinsmeister AR, Melton LJ, 3rd, Talley NJ. Natural history of functional gastrointestinal disorders: a 12-year longitudinal population-based study. Gastroenterology. 2007;133:799-807.

5. Powell R. On certain painful afflictions of the intestinal canal. Med Trans Royal Coll Phys. 1818;6: 106-17.

6. Chaudhary NA, Truelove SC. The irritable colon syndrome. A study of the clinical features, predisposing causes, and prognosis in 130 cases. Q J Med. 1962;31:307-22.

7. DeLor CJ. The irritable bowel syndrome. Am J Gastroenterol. 1967;47:427-34.

8. Manning AP, Thompson WG, Heaton KW, Morris AF. Towards positive diagnosis of the irritable bowel. BMJ. 1978;2:653-4.

9. Thompson WG, Dotevall, G., Drossman, D.A., Heaton, K.W., Kruis, W. Irritable bowel syndrome: guidelines for the diagnosis. Gastroenterol Int. 1989;2 92-5.

10. Drossman DA. Updates for Rome IV. Rome Foundation Newsletter. 2013;6(2-3).

11. Lovell RM, Ford AC. Global prevalence of and risk factors for irritable bowel syndrome: a meta-analysis. Clin Gastroenterol Hepatol 2012;10:712-21 e4.

12. Spiegel BM. The burden of IBS: looking at metrics. Curr Gastroenterol Rep. 2009;11:265-9.

13. Canavan C, West J, Card T. Review article: the economic impact of the irritable bowel syndrome. Aliment Pharmacol Ther. 2014;40:1023-34.

14. Spiegel BM, Farid M, Esrailian E, Talley J, Chang L. Is irritable bowel syndrome a diagnosis of exclusion?: a survey of primary care providers, gastroenterologists, and IBS experts. Am J Gastroenterol. 2010;105:848-58.

15. Corsetti M, Van Oudenhove L, Tack J. The quest for biomarkers in IBS-where should it lead us? Neurogastroenterol Motil. 2014;26:1669-76.

16. El-Salhy M, Hatlebakk JG, Gilja OH, Hausken T. Irritable bowel syndrome: recent developments in diagnosis, pathophysiology, and treatment. Expert Rev Gastroenterol Hepatol. 2014;8:435-43.

17. Camilleri M. Peripheral mechanisms in irritable bowel syndrome. N Engl J Med. 2012;367:1626-35.

18. Saito YA, Petersen GM, Larson JJ, Atkinson EJ, Fridley BL, de Andrade M, Locke GR 3rd, Zimmerman JM, Almazar-Elder AE, Talley NJ. Familial aggregation of irritable bowel syndrome: a family case-control study. Am J Gastroenterol. 2010;105:833-41.

19. Bengtson $M B$, Ronning $\mathrm{T}$, Vatn $\mathrm{MH}$, Harris JR. Irritable bowel syndrome in twins: genes and environment. Gut. 2006;55:1754-9.

20. van der Veek PP, van den Berg M, de Kroon YE, Verspaget HW, Masclee AA. Role of tumor necrosis factor-alpha and interleukin-10 gene polymorphisms in irritable bowel syndrome. Am J Gastroenterol. 2005;100:2510-6.

21. Van Kerkhoven LA, Laheij RJ, Jansen JB. Meta-analysis: a functional polymorphism in the gene encoding for activity of the serotonin transporter protein is not associated with the irritable bowel syndrome. Aliment Pharmacol Ther. 2007;26:979-86.

22. Camilleri M, Katzka DA. Irritable bowel syndrome: methods, mechanisms, and pathophysiology. Genetic epidemiology and pharmacogenetics in irritable bowel syndrome. Am J Physiol Gastrointest Liver Physiol. 2012;302:G1075-84.

23. Stewart GT. Post-dysenteric colitis. BMJ. 1950;1:405-9.

24. Marshall JK, Thabane M, Garg AX, Clark WF, Salvadori M, Collins SM; Walkerton Health Study Investigators. Incidence and epidemiology of irritable bowel syndrome after a large waterborne outbreak of bacterial dysentery. Gastroenterology. 2006;131:445-50.

25. Dolwani S, Metzner M, Wassell JJ, Yong A, Hawthorne AB. Diagnostic accuracy of faecal calprotectin estimation in prediction of abnormal small bowel radiology. Aliment Pharmacol Ther. 2004;20:615-21. 
26. Ohman L, Stridsberg M, Isaksson S, Jerlstad P, Simren M. Altered levels of fecal chromogranins and secretogranins in IBS: relevance for pathophysiology and symptoms? Am J Gastroenterol. 2012;107:440-7.

27. Bashashati M, Rezaei N, Shafieyoun A, McKernan DP, Chang L, Öhman L, Quigley EM, Schmulson M, Sharkey KA, Simrén M. Cytokine imbalance in irritable bowel syndrome: a systematic review and metaanalysis. Neurogastroenterol Motil. 2014;26:1036-48.

28. Dinan TG, Clarke G, Quigley EM, Scott LV, Shanahan F, Cryan J, Cooney J, Keeling PW. Enhanced cholinergic-mediated increase in the pro-inflammatory cytokine IL-6 in irritable bowel syndrome: role of muscarinic receptors. Am J Gastroenterol. 2008;103:2570-6.

29. Dinan TG, Quigley EM, Ahmed SM, Scully P, O'Brien S, O'Mahony L, O'Mahony S, Shanahan F, Keeling PW. Hypothalamic-pituitary-gut axis dysregulation in irritable bowel syndrome: plasma cytokines as a potential biomarker? Gastroenterology. 2006;130:304-11.

30. Barbara G, Stanghellini V, De Giorgio R, Cremon C, Cottrell GS, Santini D, Pasquinelli G, Morselli-Labate AM, Grady EF, Bunnett NW, Collins SM, Corinaldesi R. Activated mast cells in proximity to colonic nerves correlate with abdominal pain in irritable bowel syndrome. Gastroenterology. 2004;126:693702.

31. Vanuytsel T, van Wanrooy S, Vanheel H, Vanormelingen C, Verschueren S, Houben E, Salim Rasoel S, Tóth J, Holvoet L, Farré R, Van Oudenhove L, Boeckxstaens G, Verbeke K, Tack J. Psychological stress and corticotropin-releasing hormone increase intestinal permeability in humans by a mast celldependent mechanism. Gut. 2014;63:1293-9.

32. Turner JR. Intestinal mucosal barrier function in health and disease. Nat Rev Immunol. 2009;9:799-809.

33. Marshall JK, Thabane M, Garg AX, Clark W, Meddings J, Collins SM; WEL Investigators. Intestinal permeability in patients with irritable bowel syndrome after a waterborne outbreak of acute gastroenteritis in Walkerton, Ontario. Aliment Pharmacol Ther. 2004;20:1317-22.

34. Spiller RC, Jenkins D, Thornley JP, Hebden JM, Wright T, Skinner M, Neal KR. Increased rectal mucosal enteroendocrine cells, T lymphocytes, and increased gut permeability following acute Campylobacter enteritis and in post-dysenteric irritable bowel syndrome. Gut. 2000;47:804-11.

35. Dunlop SP, Hebden J, Campbell E, Naesdal J, Olbe L, Perkins AC, Spiller RC. Abnormal intestinal permeability in subgroups of diarrhea-predominant irritable bowel syndromes. Am J Gastroenterol. 2006;101:1288-94.

36. Rao AS, Camilleri M, Eckert DJ, Busciglio I, Burton DD, Ryks M, Wong BS, Lamsam J, Singh R, Zinsmeister AR. Urine sugars for in vivo gut permeability: validation and comparisons in irritable bowel syndromediarrhea and controls. Am J Physiol Gastrointest Liver Physiol. 2011;301:G919-28.

37. Zhou $Q$, Zhang $B$, Verne GN. Intestinal membrane permeability and hypersensitivity in the irritable bowel syndrome. Pain. 2009;146:41-6.

38. Zhou Q, Souba WW, Croce CM, Verne GN. MicroRNA-29a regulates intestinal membrane permeability in patients with irritable bowel syndrome. Gut. 2010;59:775-84.

39. Kerckhoffs AP, Akkermans LM, de Smet MB, Besselink MG, Hietbrink F, Bartelink IH, Busschers WB, Samsom M, Renooij W. Intestinal permeability in irritable bowel syndrome patients: effects of NSAIDs. Dig Dis Sci. 2010;55:716-23.

40. Del Valle-Pinero AY, Van Deventer HE, Fourie NH, Martino AC, Patel NS, Remaley AT, Henderson WA. Gastrointestinal permeability in patients with irritable bowel syndrome assessed using a four probe permeability solution. Clin Chim Acta. 2013;418:97-101.

41. Sanchez de Medina F, Romero-Calvo I, Mascaraque C, Martinez-Augustin O. Intestinal inflammation and mucosal barrier function. Inflamm Bowel Dis. 2014;20:2394-404.

42. Rajilić-Stojanović M, Biagi E, Heilig HG, Kajander K, Kekkonen RA, Tims S, de Vos WM. Global and deep molecular analysis of microbiota signatures in fecal samples from patients with irritable bowel syndrome. Gastroenterology. 2011;141:1792-801.

43. Jalanka-Tuovinen J, Salojärvi J, Salonen A, Immonen O, Garsed K, Kelly FM, Zaitoun A, Palva A, Spiller RC, de Vos WM. Faecal microbiota composition and host-microbe cross-talk following gastroenteritis and in postinfectious irritable bowel syndrome. Gut. 2014;63:1737-45.

44. Jeffery IB, O'Toole PW, Öhman L, Claesson MJ, Deane J, Quigley EM, Simrén M. An irritable bowel syndrome subtype defined by species-specific alterations in faecal microbiota. Gut. 2012;61:997-1006. 
45. Rajilić-Stojanović M, Jonkers DM, Salonen A, Hanevik K, Raes J, Jalanka J, de Vos WM, Manichanh C, Golic N, Enck P, Philippou E, Iraqi FA, Clarke G, Spiller RC, Penders J. Intestinal Microbiota And Diet in IBS: Causes, Consequences, or Epiphenomena? Am J Gastroenterol. 2015;110:278-87.

46. Ford AC, Quigley EM, Lacy BE, Lembo AJ, Saito YA, Schiller LR, Soffer EE, Spiegel BM, Moayyedi P. Efficacy of prebiotics, probiotics, and synbiotics in irritable bowel syndrome and chronic idiopathic constipation: systematic review and meta-analysis. Am J Gastroenterol. 2014;109:1547-61.

47. Karczewski J, Troost FJ, Konings I, Dekker J, Kleerebezem M, Brummer RJ, Wells JM. Regulation of human epithelial tight junction proteins by Lactobacillus plantarum in vivo and protective effects on the epithelial barrier. Am J Physiol Gastrointest Liver Physiol. 2010;298:G851-9.

48. Mao Y, Nobaek S, Kasravi B, Adawi D, Stenram U, Molin G, Jeppsson B. The effects of Lactobacillus strains and oat fiber on methotrexate-induced enterocolitis in rats. Gastroenterology. 1996;111:334-44.

49. White JS, Hoper M, Parks RW, Clements WD, Diamond T, Bengmark S. The probiotic bacterium Lactobacillus plantarum species 299 reduces intestinal permeability in experimental biliary obstruction. Lett Appl Microbiol. 2006;42:19-23.

50. Mennigen R, Nolte K, Rijcken E, Utech M, Loeffler B, Senninger N, Bruewer M. Probiotic mixture VSL\#3 protects the epithelial barrier by maintaining tight junction protein expression and preventing apoptosis in a murine model of colitis. Am J Physiol Gastrointest Liver Physiol. 2009;296:G1140-9.

51. Mangell $P$, Nejdfors $P$, Wang M, Ahrné S, Weström B, Thorlacius H, Jeppsson B. Lactobacillus plantarum 299v inhibits Escherichia coli-induced intestinal permeability. Dig Dis Sci. 2002;47:511-6.

52. Zeng J, Li YQ, Zuo XL, Zhen YB, Yang J, Liu CH. Clinical trial: effect of active lactic acid bacteria on mucosal barrier function in patients with diarrhoea-predominant irritable bowel syndrome. Alimentary pharmacology \& therapeutics. 2008 Oct 15;28(8):994-1002.

53. Ludidi S, Conchillo JM, Keszthelyi D, Van Avesaat M, Kruimel JW, Jonkers DM, Masclee AA. Rectal hypersensitivity as hallmark for irritable bowel syndrome: defining the optimal cutoff. Neurogastroenterol Motil. 2012;24:729-33, e345-6.

54. Ritchie J. Pain from distension of the pelvic colon by inflating a balloon in the irritable colon syndrome. Gut. 1973;14:125-32.

55. Keszthelyi D, Troost FJ, Masclee AA. Irritable bowel syndrome: methods, mechanisms, and pathophysiology. Methods to assess visceral hypersensitivity in irritable bowel syndrome. Am J Physiol Gastrointest Liver Physiol. 2012;303:G141-54.

56. Ludidi S, Jonkers DM, Koning CJ, Kruimel JW, Mulder L, van der Vaart IB, Conchillo JM, Masclee AA. Randomized clinical trial on the effect of a multispecies probiotic on visceroperception in hypersensitive IBS patients. Neurogastroenterol Motil. 2014;26:705-14.

57. Mertz H, Naliboff B, Munakata J, Niazi N, Mayer EA. Altered rectal perception is a biological marker of patients with irritable bowel syndrome. Gastroenterology. 1995;109:40-52.

58. Walker KM, Urban L, Medhurst SJ, Patel S, Panesar M, Fox AJ, Mclntyre P. The VR1 antagonist capsazepine reverses mechanical hyperalgesia in models of inflammatory and neuropathic pain. J Pharmacol Exp Ther. 2003;304:56-62.

59. Caterina MJ, Schumacher MA, Tominaga M, Rosen TA, Levine JD, Julius D. The capsaicin receptor: a heat-activated ion channel in the pain pathway. Nature. 1997;389:816-24.

60. Akbar A, Yiangou Y, Facer P, Walters JR, Anand P, Ghosh S. Increased capsaicin receptor TRPV1expressing sensory fibres in irritable bowel syndrome and their correlation with abdominal pain. Gut. 2008;57:923-9.

61. Camilleri M, Lasch K, Zhou W. Irritable bowel syndrome: methods, mechanisms, and pathophysiology. The confluence of increased permeability, inflammation, and pain in irritable bowel syndrome. Am J Physiol Gastrointest Liver Physiol. 2012;303:G775-85.

62. Dickhaus B, Mayer EA, Firooz N, Stains J, Conde F, Olivas TI, Fass R, Chang L, Mayer M, Naliboff BD. Irritable bowel syndrome patients show enhanced modulation of visceral perception by auditory stress. Am J Gastroenterol. 2003;98:135-43.

63. Accarino AM, Azpiroz F, Malagelada JR. Attention and distraction: effects on gut perception. Gastroenterology. 1997;113:415-22.

64. Schmid J, Langhorst J, Gaß F, Theysohn N, Benson S, Engler H, Gizewski ER, Forsting M, Elsenbruch S. Placebo analgesia in patients with functional and organic abdominal pain: a fMRI study in IBS, UC and healthy volunteers. Gut. 2015;64:418-27. 
65. Wilder-Smith $\mathrm{CH}$, Schindler D, Lovblad K, Redmond SM, Nirkko A. Brain functional magnetic resonance imaging of rectal pain and activation of endogenous inhibitory mechanisms in irritable bowel syndrome patient subgroups and healthy controls. Gut. 2004;53:1595-601.

66. Fadgyas-Stanculete M, Buga AM, Popa-Wagner A, Dumitrascu DL. The relationship between irritable bowel syndrome and psychiatric disorders: from molecular changes to clinical manifestations. J Mol Psychiatry. 2014;2:4.

67. Mayer EA, Craske M, Naliboff BD. Depression, anxiety, and the gastrointestinal system. J Clin Psychiatry. 2001;62 Suppl 8:28-36; discussion 7.

68. Kaplan DS, Masand PS, Gupta S. The relationship of irritable bowel syndrome (IBS) and panic disorder. Ann Clin Psychiatry. 1996;8:81-8.

69. Fukudo S, Nomura T, Hongo M. Impact of corticotropin-releasing hormone on gastrointestinal motility and adrenocorticotropic hormone in normal controls and patients with irritable bowel syndrome. Gut. 1998;42:845-9.

70. Heitkemper M, Burr RL, Jarrett M, Hertig V, Lustyk MK, Bond EF. Evidence for autonomic nervous system imbalance in women with irritable bowel syndrome. Dig Dis Sci. 1998;43:2093-8.

71. Keszthelyi D, Troost FJ, Masclee AA. Understanding the role of tryptophan and serotonin metabolism in gastrointestinal function. Neurogastroenterol Motil. 2009;21:1239-49.

72. Cumming W. Electro-galvanism in a peculiar affliction of the mucous membrane of the bowels. London Med Gazette. 1849;NS9:969-73.

73. Ford AC, Moayyedi P. Meta-analysis: factors affecting placebo response rate in the irritable bowel syndrome. Aliment Pharmacol Ther. 2010;32:144-58.

74. U.S. Department of Health and Human Services FaDA, Center for Drug Evaluation and Research (CDER). Guidance for Industry Irritable Bowel Syndrome - Clinical Evaluation of Drugs for Treatment, 2012. http://wwwfdagov/downloads/Drugs/Guidances/UCM205269pdf.

75. Spiegel B, Bolus R, Harris LA, Lucak S, Naliboff B, Esrailian E, Chey WD, Lembo A, Karsan H, Tillisch K, Talley J, Mayer E, Chang L. Measuring irritable bowel syndrome patient-reported outcomes with an abdominal pain numeric rating scale. Aliment Pharmacol Ther. 2009;30:1159-70.

76. Svedlund J, Sjodin I, Dotevall G. GSRS--a clinical rating scale for gastrointestinal symptoms in patients with irritable bowel syndrome and peptic ulcer disease. Dig Dis Sci. 1988;33:129-34.

77. Francis CY, Morris J, Whorwell PJ. The irritable bowel severity scoring system: a simple method of monitoring irritable bowel syndrome and its progress. Aliment Pharmacol Ther. 1997;11:395-402.

78. Stone AA, Shiffman S, Schwartz JE, Broderick JE, Hufford MR. Patient compliance with paper and electronic diaries. Control Clin Trials. 2003;24:182-99.

79. Biomarkers Definitions Working G. Biomarkers and surrogate endpoints: preferred definitions and conceptual framework. Clin Pharmacol Ther. 2001;69:89-95.

80. Lembo AJ, Neri B, Tolley J, Barken D, Carroll S, Pan H. Use of serum biomarkers in a diagnostic test for irritable bowel syndrome. Aliment Pharmacol Ther. 2009;29:834-42.

81. Jones MP, Chey WD, Singh S, Gong H, Shringarpure R, Hoe N, Chuang E, Talley NJ. A biomarker panel and psychological morbidity differentiates the irritable bowel syndrome from health and provides novel pathophysiological leads. Aliment Pharmacol Ther. 2014;39:426-37. 


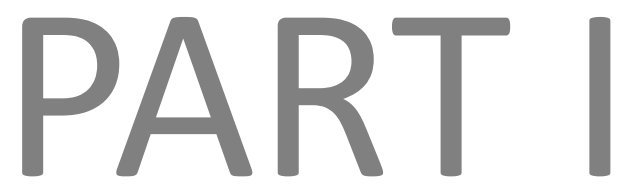

Pathophysiology 


\section{Chapter 2

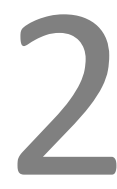

Intestinal permeability in IBS

Small intestinal permeability is increased in diarrhoea predominant IBS, while alterations in gastroduodenal permeability in all IBS subtypes are largely attributable to confounders

Zlatan Mujagic ${ }^{*}$, Samefko Ludidi ${ }^{*}$, Daniel Keszthelyi, Martine AM Hesselink, Joanna W Kruimel, Kaatje Lenaerts, Nordin MJ Hanssen, José M Conchillo, Daisy MAE Jonkers, Ad AM Masclee

${ }^{*}$ Both authors contributed equally to the manuscript

Alimentary Pharmacology \& Therapeutics 2014;40:288-97 


\section{Abstract}

\section{Background}

Intestinal permeability has been studied in small groups of IBS patients with contrasting findings.

\section{Aim}

The aims of the present study were to assess intestinal permeability at different sites of the GI tract in different subtypes of well-characterised IBS patients and healthy controls (HC) and to assess potential confounding factors.

\section{Methods}

IBS patients and HC underwent a multi-sugar test to assess site-specific intestinal permeability. Sucrose excretion and lactulose/rhamnose ratio in 0-5h urine indicated gastroduodenal and small intestinal permeability, respectively. Sucralose/erythritol ratio in $0-24 \mathrm{~h}$ and $5-24 \mathrm{~h}$ urine indicated whole gut and colonic permeability, respectively. Linear regression analysis was used to assess the association between IBS groups and intestinal permeability and to adjust for age, sex, BMI, anxiety or depression, smoking, alcohol intake, and use of medication.

\section{Results}

91 IBS patients, i.e. $37 \%$ IBS-D, 23\% IBS-C, 33\% IBS-M and 7\% IBS-U, and 94 HC were enrolled. Urinary sucrose excretion was significantly increased in the total IBS group ( $\mu$ mol, median [Q1;Q3]: 5.26 [1.82;11.03] vs. 2.44 [0.91;5.85], $\mathrm{p}<0.05$ ), as well as in IBS$C$ and IBS-D versus HC. However, differences attenuated when adjusting for confounders. The lactulose/rhamnose ratio was increased in IBS-D versus HC $(0.023$ [0.013;0.038] vs. 0.014 [0.008;0.025], p<0.05), which remained significant after adjustment for confounders. No difference was found in 0-24 and 5-24 hour sucralose/erythritol ratio between groups.

\section{Conclusion}

Small intestinal permeability is increased in patients with IBS-D compared to HC, irrespective of confounding factors. Adjustment for confounders is necessary when studying intestinal permeability, especially in a heterogeneous disorder such as IBS. 


\section{Introduction}

Irritable bowel syndrome (IBS) is a frequently occurring functional disorder of the gastrointestinal (GI) tract, characterised by abdominal pain and alterations in bowel habits. It is associated with impaired quality of life, increased use of health care services and high health care costs. ${ }^{1,2}$ The IBS patient population is phenotypically very heterogeneous, with a diversity of predominant symptoms and clinical characteristics, ${ }^{3}$ reflecting the multitude of underlying pathophysiological mechanisms. ${ }^{4}$

Changes in gut epithelial barrier function have been observed in IBS and have been postulated to play a role in the pathogenesis of the disorder. Several studies have pointed to an increased intestinal permeability in IBS patients when compared to healthy controls. However, these findings were based on data obtained in small study populations, $^{5-7}$ or only in specific IBS subpopulations, such as post-infectious IBS cohorts $^{7-9}$ or diarrhoea predominant IBS (IBS-D). ${ }^{5,10,11}$ Alterations in intestinal permeability appear to be subtype-related and site-specific, as several studies found an increased small intestinal permeability in patients with IBS-D. ${ }^{5,7,10,11}$ Data on colonic and whole gut permeability are inconsistent ${ }^{5-7,12,13}$ and only a limited number of studies investigated a non-selected heterogeneous IBS population ${ }^{6,12,14}$ or focussed also on patients with constipation predominant IBS (IBS-C). ${ }^{7,13}$ Comparison of data from published studies is further hampered by differences in methodology of permeability tests. Furthermore, factors such as demographical characteristics, medication and lifestyle are known to influence intestinal permeability, but currently available information on possible confounding effects by these factors on intestinal permeability in IBS patients is very limited. ${ }^{8,12}$

Therefore, data are needed from larger well-characterised IBS cohorts using validated site-specific intestinal permeability measurements for the different anatomical GI locations, comparing different subtypes and taking possible confounders into account.

We hypothesized that 1) IBS patients have increased intestinal permeability compared to healthy controls ( $\mathrm{HC})$, which is site-specific and subtype-dependent and 2 ) intestinal permeability in IBS patients is affected by factors such as demographical or lifestyle characteristics, psychological symptoms and use of medication.

Aims of the present study were 1) to assess intestinal permeability of different sites of the GI tract, in a large group of well-characterised IBS patients and healthy controls and to investigate whether differences exist between IBS subtypes and 2) to assess potential confounding effects of demographical characteristics, psychological and lifestyle factors and use of medication on intestinal permeability. 


\section{Materials and methods}

\section{Study participants}

The present study is part of a larger cohort study on the phenotypical and genotypical characterisation of patients with IBS, with age and gender matched healthy controls, wherein multiple parameters were assessed according to standardised procedures. The subgroup of patients and controls that underwent an intestinal permeability test was included in the current study.

Between September 2009 and June 2013, subjects between 18 and 75 years of age, with IBS diagnosed by the Rome III criteria were recruited via the outpatient department of the division Gastroenterology-Hepatology of the Maastricht University Medical Center+, a secondary and tertiary referral centre. Medical history was taken by a gastroenterologist. GI endoscopy with biopsies, abdominal imaging by ultrasonography or CT scan and/or blood, breath and faecal analysis were performed to exclude organic disease, if indicated. Patients with a history of abdominal surgery, except appendectomy, laparoscopic cholecystectomy and hysterectomy, and patients who were not able to abide the instructions for the permeability test were excluded. IBS patients were assigned to subtypes, i.e. diarrhoea (IBS-D), constipation (IBS-C), mixed stool pattern (IBS-M) and unspecified stool pattern (IBS-U), based on predominant bowel habits according to the ROME III criteria and daily Bristol Stool Chart in a 14-day symptom dairy. ${ }^{15}$

Healthy subjects in the same age category were included, as controls (HC). They were enrolled via public advertising on websites and in local newspapers. Before participation, a brief medical history was taken to exclude the presence of GI disorders and current or previous GI complaints. All measurements performed in IBS patients were also carried out in $\mathrm{HC}$.

All subjects have given written informed consent prior to their inclusion. The study protocol has been approved by the Maastricht University Medical Center+ Committee of Ethics and was executed according to the revised Declaration of Helsinki $\left(59^{\text {th }}\right.$ general assembly of the WMA, Seoul, South Korea, Oct. 2008). The study has been registered in the US National Library of Medicine (http://www.clinicaltrials.gov, NCT00775060).

\section{Permeability measurement}

Intestinal permeability was assessed on four sites of the GI tract. Sucrose excretion and the lactulose and rhamnose (L/R) ratio in 0-5 hour urine were used as indicators of gastroduodenal and small intestinal permeability, respectively. Whole-gut permeability was indicated by the sucralose and erythritol (S/E) ratio in 0-24 hour urine, whereas the 5-24 hour S/E ratio was used as indicator for colonic permeability (Figure 2.1). ${ }^{16-18}$ 


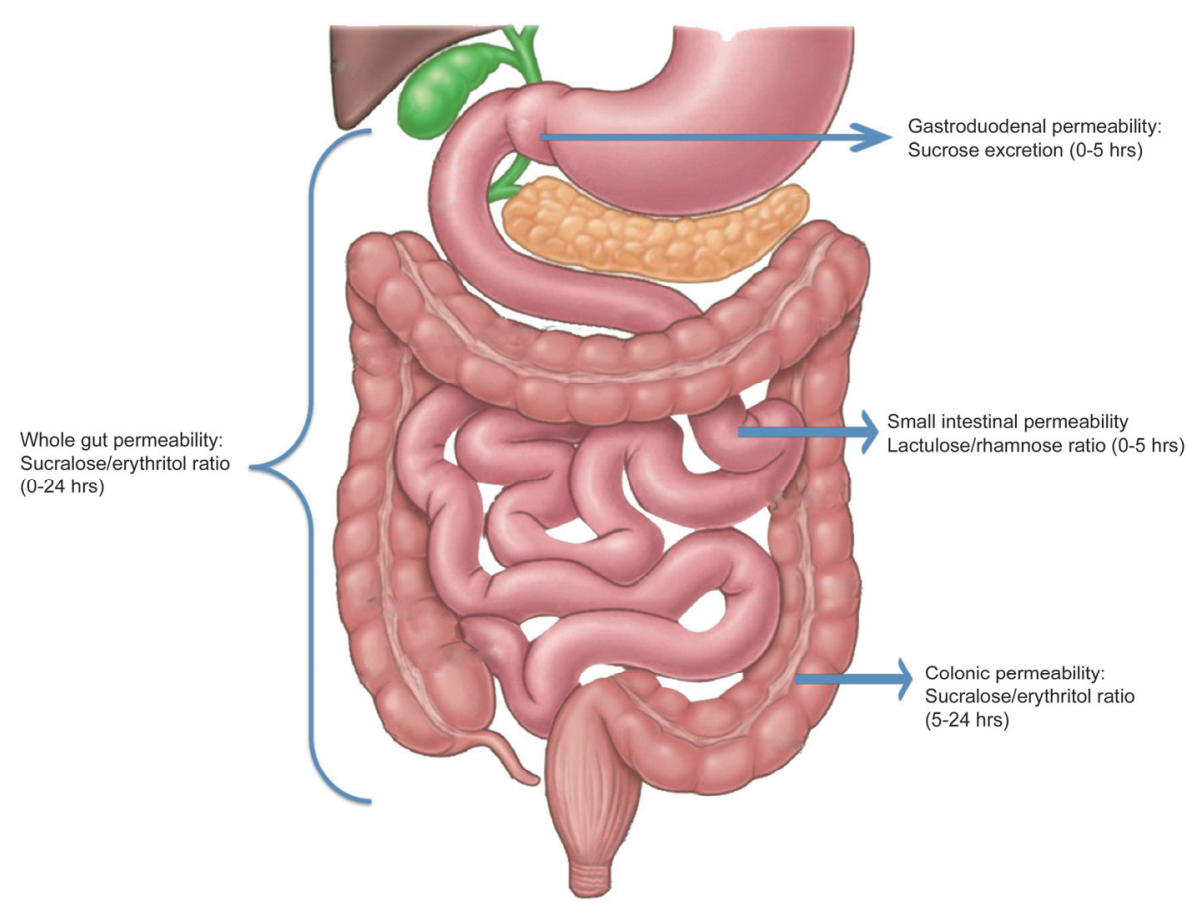

Figure 2.1 Multi-sugar test, indicating permeability of four sites of the GI tract.

All patients and $\mathrm{HC}$ included in this study underwent a non-invasive validated multisugar test, measuring urinary excretion of ingested sugar probes indicating gastroduodenal, small intestinal, colonic and whole gut permeability, as previously validated by van Wijck et al. ${ }^{17,18}$ During and two days prior to the test day excessive physical exercise, consumption of alcohol and intake of non-steroidal anti-inflammatory drugs (NSAIDs) were prohibited, to prevent short term effects of these factors on intestinal permeability.

After an overnight fast, participants emptied their urinary bladder and consumed a multi-sugar drink (150 ml of tap water) containing $1 \mathrm{~g}$ sucrose (van Gilse, Dinteloord, the Netherlands), $1 \mathrm{~g}$ lactulose (Centrafarm, Etten-Leur, the Netherlands), $0.5 \mathrm{~g}$ L-rhamnose (Danisco, Copenhagen, Denmark), 1 g sucralose (Brenntag, Sittard, the Netherlands) and $1 \mathrm{~g}$ erythritol (Now Foods, Bloomindale, USA). Patients were requested to collect 24 hour urine in two separate fractions, i.e. 0-5 and 5-24 hour fraction. During the first 5 hours of urine collection, neither foods nor drinks were allowed with the exception of water. Total urine volume was determined and urine samples were aliquoted and frozen at $-80^{\circ} \mathrm{C}$ until analyses of the sugar probes by HPLCMS as previously described. ${ }^{17,18}$ 


\section{Questionnaires}

Within a period of two weeks prior to the permeability measurement, questionnaires on demographical characteristics, lifestyle factors and the use of medication were completed by the participants. The presence of GI symptoms was assessed using the validated gastrointestinal symptom rating scale (GSRS), consisting of 16 items clustered into five major Gl syndromes: abdominal pain, reflux, diarrhea, indigestion and constipation syndrome. ${ }^{19}$ Moreover, symptoms of discomfort, abdominal pain, bloating, flatulence, nausea and intensity of total complaints were scored daily during 14 days, on a 5-point Likert-scale, in a symptom diary. Subsequently, the mean symptom composite score (MSS) was calculated, based on sum of individual symptoms per day. $^{20}$

Symptoms of anxiety and depression were assessed using the hospital anxiety and depression scale (HADS). This questionnaire consists of two 7-item scales, that is one for anxiety and one for depression, each with a Likert scoring range between $0-3$. A higher score indicates a higher degree of distress and a total score $\geq 8$ on either the anxiety or depression scale is indicative for the presence of anxiety or depression symptoms, respectively. ${ }^{21,22}$

\section{Data and statistical analysis}

Statistical analysis was performed using IBM SPSS Statistics version 21.0 (IBM Statistics for Macintosh, Chicago IL).

The total IBS group is referred to as IBS TOTAL. Data of IBS TOTAL and IBS subtypes versus HC were compared using linear regression analysis. Due to skewed distribution, urinary sugar excretions and ratios were natural logarithmic (LN) transformed. Linear regression analysis was performed to assess the association between presence of IBS and intestinal permeability (Model 1). Furthermore, we investigated possible confounding effects of disease-related factors on intestinal permeability. Therefore, we first adjusted the crude analysis for demographical characteristics (age, sex, BMI), (Model 2). Next, we additionally adjusted for psychological (anxiety or depressive) symptoms, (Model 3). We then additionally adjusted for lifestyle factors (smoking history, defined as current or previous smoker, and high use of alcohol, defined as more than 15 units per week), (Model 4), and thereafter additionally for use of medication in the two weeks prior to inclusion (NSAID, proton pump inhibitors (PPI), selective serotonin reuptake inhibitors (SSRI) and medication that affects motility, i.e. laxatives, prokinetics, opioids, spasmolytics and anti-diarrhoeal drugs), (Model 5). All analyses

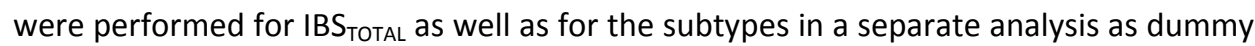
variables. To investigate which covariate had the strongest confounding effect on the association between presence of IBS and sugar excretion or ratios, we additionally adjusted the crude analysis (Model 1) for all factors separately. The factors with the strongest significant effects where thereafter omitted separately from the fully 
adjusted model (Model 1-5) to determine if the factors are indeed confounders and affect the association between IBS and sugar excretion or ratios or if the factors are independently of the presence of IBS associated with sugar excretion or ratios.

Missing values were excluded listwise. A two-sided p-value of 0.05 was considered statistically significant. The $ß$ of the linear regression analyses signifies one LN change in sugar excretion and ratios versus HC.

Finally, Spearman's rank was used to assess correlation between urinary sugar excretions or ratios and GI symptoms.

\section{Results}

\section{Demographical characteristics}

Intestinal permeability data were available from 91 IBS patients and $94 \mathrm{HC}$. Predominant bowel habits for diarrhoea (IBS-D), constipation (IBS-C), mixed stool pattern (IBS-M) and unspecified stool pattern (IBS-U) were seen in $37 \%(n=34), 23 \%$ $(n=21), 33 \%(n=30)$ and $7 \%(n=6)$ of the patients, respectively. Due to the small number of patients with IBS-U, this group has not been analysed as a separate subtype. All patients were included in the analysis of the group IBS TOTAL. $_{\text {. }}$

Demographical characteristics, GI and psychological symptoms, lifestyle parameters and use of medication in $\mathrm{HC}, \mathrm{IBS}_{\text {TOTAL }}$ and IBS subtypes are presented in Table 2.1. No statistically significant differences were observed for age, sex and BMI between groups. Symptoms of anxiety and depression, as well as use medication were significantly higher in IBS TOTAL $_{\text {versus }} \mathrm{HC}$. Overall alcohol consumption was significantly lower in $\mathrm{IBS}_{\text {TOTAL }}$ when compared to $\mathrm{HC}$, and this was also true for high alcohol intake, i.e. above 15 units per week. As to be expected, severity of all Gl symptoms was significantly higher in IBS patients versus HC and bowel habits were altered according to IBS subtype.

\section{Urinary sugar excretion}

Data of absolute recovery of urinary excreted sugars per urine fraction is provided in Supplementary Table S2.1. 
Table 2.1 Baseline characteristics in healthy controls $(\mathrm{HC})$, IBS patients (IBS TOTAL $_{\text {) }}$ and IBS subtypes (i.e. IBSD, IBS-C and IBS-M).

\begin{tabular}{|c|c|c|c|c|c|}
\hline Parameter & $\begin{array}{c}\mathrm{HC} \\
(n=94)\end{array}$ & $\begin{array}{c}\mathrm{IBS}_{\text {TOTAL }} \\
(\mathrm{n}=91)\end{array}$ & $\begin{array}{l}\text { IBS-D } \\
(n=34)\end{array}$ & $\begin{array}{c}\text { IBS-C } \\
(n=21)\end{array}$ & $\begin{array}{l}\text { IBS-M } \\
(n=30)\end{array}$ \\
\hline \multicolumn{6}{|l|}{ Demographical characteristics } \\
\hline Age (mean years \pm SEM) & $45.0 \pm 2.0$ & $44.4 \pm 1.6$ & $45.0 \pm 2.4$ & $47.5 \pm 3.6$ & $42.2 \pm 3.1$ \\
\hline Female sex (\%) & 58.5 & 63.7 & 57.1 & 61.9 & 70 \\
\hline $\mathrm{BMI}$ (mean $\mathrm{kg} / \mathrm{m}^{2} \pm \mathrm{SEM}$ ) & $23.8 \pm 0.5$ & $25.2 \pm 0.6$ & $25.6 \pm 1.2$ & $25.0 \pm 0.9$ & $25.4 \pm 0.7$ \\
\hline \multicolumn{6}{|l|}{ Anxiety and depressive symptoms (HADS $\geq 8)$} \\
\hline Depressive symptoms (\%) & 7.7 & $20.5^{\mathrm{a}}$ & $28.6^{\mathrm{a}}$ & 20.0 & 17.1 \\
\hline Anxiety symptoms (\%) & 12.1 & $39.8^{c}$ & $45.7^{\mathrm{c}}$ & $35.0^{\mathrm{a}}$ & 35. $7^{\mathrm{a}}$ \\
\hline Depr. and/or anxiety symptoms (\%) & 14.9 & $44.9^{c}$ & $54.3^{c}$ & $35.0^{\mathrm{a}}$ & $39.3^{\mathrm{a}}$ \\
\hline \multicolumn{6}{|l|}{ Lifestyle parameters } \\
\hline Positive smoking history (\%) & 42.6 & 51.6 & $62.9^{a}$ & 57.1 & 36.7 \\
\hline Alcohol intake: 0 units per week (\%) & 16.3 & $40.9^{c}$ & $40.0^{b}$ & 30.0 & $46.4^{b}$ \\
\hline Alcohol intake: $>15$ units per week (\%) & 11.7 & $1.1^{b}$ & 2.9 & 0.0 & $0.0^{\mathrm{a}}$ \\
\hline \multicolumn{6}{|c|}{ Use of medication 2 weeks prior to participation " } \\
\hline PPI (\%) & 2.1 & $28.6^{c}$ & $31.4^{\mathrm{c}}$ & $23.8^{b}$ & $33.3^{c}$ \\
\hline NSAID (\%) & 5.3 & $14.3^{*}$ & 8.6 & 19.0 & 16.7 \\
\hline SSRI (\%) & 1.1 & $15.4^{\mathrm{c}}$ & $17.1^{\mathrm{b}}$ & 0.0 & $23.3^{c}$ \\
\hline Motility + drugs (\%) & 0.0 & $19.8^{c}$ & $14.3^{\mathrm{a}}$ & $23.8^{c}$ & $26.7^{c}$ \\
\hline Motility - drugs (\%) & 0.0 & $12.1^{\mathrm{c}}$ & $17.1^{\mathrm{c}}$ & 9.5 & $10.0^{\mathrm{a}}$ \\
\hline Statins (\%) & 5.3 & 8.8 & 5.7 & 14.3 & 6.7 \\
\hline Antihypertensive med. (\%) & 11.7 & 16.5 & 14.3 & 23.8 & 16.7 \\
\hline \multicolumn{6}{|l|}{ GSRS symptom scores (Mean \pm SEM) } \\
\hline Abdominal pain & $1.6 \pm 0.1$ & $3.2 \pm 0.1^{\mathrm{c}}$ & $3.3 \pm 0.2^{c}$ & $3.3 \pm 0.3^{c}$ & $3.2 \pm 0.2^{c}$ \\
\hline Reflux syndrome & $1.2 \pm 0.1$ & $2.2 \pm 0.2^{c}$ & $2.3 \pm 0.3^{c}$ & $2.2 \pm 0.3^{c}$ & $2.3 \pm 0.3^{c}$ \\
\hline Diarrhoea syndrome & $1.4 \pm 0.1$ & $3.6 \pm 0.2^{c}$ & $4.0 \pm 0.2^{c}$ & $2.8 \pm 0.3^{c}$ & $3.7 \pm 0.3^{c}$ \\
\hline Indigestion syndrome & $1.9 \pm 0.1$ & $4.4 \pm 0.1^{\mathrm{c}}$ & $4.2 \pm 0.2^{c}$ & $3.9 \pm 0.3^{c}$ & $4.2 \pm 0.2^{c}$ \\
\hline Constipation syndrome & $1.6 \pm 0.1$ & $3.4 \pm 0.2^{c}$ & $2.7 \pm 0.2^{c}$ & $3.9 \pm 0.4^{c}$ & $3.8 \pm 0.2^{c}$ \\
\hline \multicolumn{6}{|c|}{ Diary symptom scores - 14 day average (Mean \pm SEM) } \\
\hline Discomfort & $1.1 \pm 0.1$ & $2.5 \pm 0.1^{c}$ & $2.4 \pm 0.1^{c}$ & $2.5 \pm 0.2^{c}$ & $2.5 \pm 0.2^{c}$ \\
\hline Abdominal pain & $1.1 \pm 0.1$ & $2.3 \pm 0.1^{c}$ & $2.2 \pm 0.1^{c}$ & $2.5 \pm 0.2^{c}$ & $2.2 \pm 0.2^{c}$ \\
\hline Bloating & $1.1 \pm 0.1$ & $2.2 \pm 0.1^{\mathrm{c}}$ & $2.2 \pm 0.2^{c}$ & $2.2 \pm 0.2^{c}$ & $2.4 \pm 0.2^{b}$ \\
\hline Flatulence & $1.3 \pm 0.1$ & $2.4 \pm 0.1^{\mathrm{c}}$ & $2.6 \pm 0.2^{c}$ & $2.1 \pm 0.1^{c}$ & $2.5 \pm 0.2^{c}$ \\
\hline Intensity of symptoms & $1.1 \pm 0.1$ & $2.5 \pm 0.1^{\mathrm{c}}$ & $2.4 \pm 0.1^{c}$ & $2.5 \pm 0.2^{c}$ & $2.5 \pm 0.2^{c}$ \\
\hline Mean symptom composite score & $6.7 \pm 0.2$ & $13.3 \pm 0.5^{c}$ & $13.2 \pm 0.8^{\mathrm{c}}$ & $13.4 \pm 0.9^{c}$ & $13.6 \pm 1.0^{c}$ \\
\hline
\end{tabular}

Motility + drugs: medication that positively affect motility, i.e. laxatives and prokinetic drugs; motility - drugs: medication that negatively affect motility, i.e. opioids, spasmolytics and anti-diarrhoeal drugs. GSRS scores presented for 5 subscales. End-of-day symptom diary scores presented as day average over 14 days, and the mean symptom composite score was calculated, based on sum of individual symptoms in the diary per day.

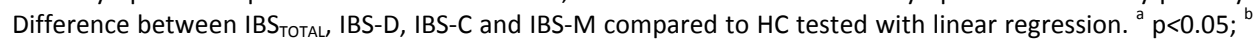
$p<0.01 ;{ }^{c} p<0.001$ vs. HC

\section{Gastroduodenal permeability}

In Table 2.2, median and ranges of urinary sucrose excretion (in 0-5h urine fraction) are given for HC, IBS TOTAL, IBS-D, IBS-C and IBS-M. Linear regression analysis of sucrose excretion with adjustments for possible confounders is provided as Supplementary Table S2.2A. 
Table 2.2 Permeability test: sugar excretion and ratios of excreted sugars as measured in urine (in 0-5, 524 and 0-24 hours fraction), for HC, IBS TOTAL, IBS-D, IBS-C and IBS-M.

\begin{tabular}{lccccc}
\hline Sugar excretion & $\begin{array}{c}\mathrm{HC} \\
(\mathrm{n}=94)\end{array}$ & $\begin{array}{c}\text { IBS }_{\text {TOTAL }} \\
(\mathrm{n}=91)\end{array}$ & $\begin{array}{c}\text { IBS-D } \\
(\mathrm{n}=34)\end{array}$ & $\begin{array}{c}\text { IBS-C } \\
(\mathrm{n}=21)\end{array}$ & $\begin{array}{c}\text { IBS-M } \\
(\mathrm{n}=30)\end{array}$ \\
\hline 0-5h Sucrose $(\mu \mathrm{mol})$ & 2.44 & 5.26 & 4.22 & 7.40 & 6.59 \\
& {$[0.91 ; 5.85]$} & {$[1.82 ; 11.03]^{\mathrm{b}}$} & {$[2.12 ; 8.03]^{\mathrm{a}}$} & {$[2.37 ; 18.29]^{\mathrm{b}}$} & {$[1.08 ; 12.13]$} \\
& 0.014 & 0.018 & 0.023 & 0.018 & 0.016 \\
0-5h L/R ratio & {$[0.008 ; 0.025]$} & {$[0.011 ; 0.029]$} & {$[0.013 ; 0.038]^{\mathrm{a}}$} & {$[0.009 ; 0.032]$} & {$[0.011 ; 0.024]$} \\
& 0.010 & 0.008 & 0.008 & 0.009 & 0.008 \\
\hline 5-24h S/E ratio & {$[0.006 ; 0.025]$} & {$[0.004 ; 0.017]$} & {$[0.004 ; 0.020]$} & {$[0.003 ; 0.015]$} & {$[0.006 ; 0.024]$} \\
& 0.009 & 0.009 & 0.009 & 0.008 & 0.010 \\
\hline 0-24h S/E ratio & {$[0.006 ; 0.013]$} & {$[0.006 ; 0.014]$} & {$[0.004 ; 0.014]$} & {$[0.004 ; 0.014]$} & {$[0.006 ; 0.016]$} \\
\hline
\end{tabular}

Results, not adjusted for confounders, presented as: median [interquartile range (IQR), i.e. Q1; Q3]. Differences, relative to $\mathrm{HC}$, tested with linear regression. Skewed variables were $\mathrm{LN}$ transformed prior to analysis. ${ }^{\mathrm{a}} \mathrm{p}<0.05,{ }^{\mathrm{b}} \mathrm{p}<0.01$ vs. $\mathrm{HC}$

Crude analysis showed a significantly higher sucrose excretion in IBS TOTAL $_{\text {(median [Q1; }}$ Q3] in $\mu$ mol: 5.26 [1.82; 11.03] $p=0.007)$, IBS-D (4.22 [2.12; 8.03] $p=0.049)$ and IBS-C (7.40 [2.37; 18.29] $\mathrm{p}=0.004)$ compared to $\mathrm{HC}(2.44$ [0.91; 5.85]). After adjustment for sex, age and $\mathrm{BMI}$, sucrose excretion remained significantly higher in $\mathrm{IBS}_{\text {TOTAL }}$ relative to $\mathrm{HC}$, ( $\beta$ : $0.49,95 \% \mathrm{Cl}: 0.05 ; 0.94, p=0.031)$. This association was no longer significant when we additionally adjusted for psychological symptoms ( $\beta$ : $0.44,95 \% \mathrm{Cl}:-0.04 ; 0.91$, $p=0.073)$, for lifestyle factors $(\beta$ : $0.37,95 \% \mathrm{Cl}:-0.12 ; 0.86, p=0.133$ ) and almost completely attenuated after additional adjustment for use of medication ( $\beta$ : $-0.04,95 \%$ $\mathrm{Cl}$ : $-0.59 ; 0.51, \mathrm{p}=0.880$ ). Overall, we found similar results for the IBS subtypes relative to $\mathrm{HC}$. Although associations appeared to be stronger within the IBS-C group, these were no longer significant after adjustment for medication use $(\beta: 0.41,95 \% \mathrm{Cl}$ : -0.39 ; 1.20, $\mathrm{p}=0.312$ ).

When separately adjusting the crude analysis for the possible confounders, several factors were associated with higher sucrose excretion. A higher $\mathrm{BMI}(\beta: 0.07,95 \% \mathrm{Cl}$ : $0.02 ; 0.11, p=0.004)$, as well as a positive smoking history $(\beta: 0.46,95 \% \mathrm{Cl}: 0.02 ; 0.89$, $p=0.040)$, were related to higher sucrose excretion. Male sex $(\beta: 0.43,95 \% \mathrm{Cl}:-0.01$; $0.87, p=0.056)$ and use of PPI's ( $\beta: 0.59,95 \% \mathrm{Cl}:-0.08 ; 1.25, p=0.084)$ were associated with higher sucrose excretion as well, but did not reach statistical significance. Drugs that positively affect motility, i.e. laxatives and prokinetics, showed the strongest association with sucrose excretion ( $\beta: 0.77,95 \% \mathrm{Cl}: 0.02 ; 0.152, \mathrm{p}=0.045)$.

Thereafter we omitted separately BMI, smoking, sex, use of PPI, laxatives and prokinetics from the fully adjusted model. When omitting BMI and the use of laxatives and prokinetics the association between sucrose excretion with IBS TOTAL $_{\text {Ta }}$ well as with the IBS subtypes became stronger again, demonstrating the confounding effect of these factors in our population, while the other three factors did not affect the analysis 
(data not shown) and are therefore not confounders in this analysis but are independently associated with increased sucrose excretion.

\section{Small intestinal permeability}

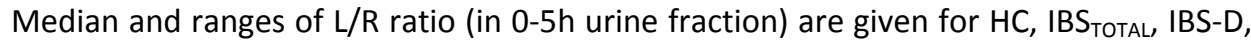
IBS-C and IBS-M in Table 2.2. Linear regression analysis of $L / R$ ratio with adjustments for possible confounders is provided as Supplementary Table S2.2B.

Crude analysis showed a significantly higher L/R ratio in IBS-D (median [Q1; Q3]: 0.023 [0.013; 0.038], $\mathrm{p}=0.029)$ compared to HC $(0.014$ [0.008; 0.025]), but no significant

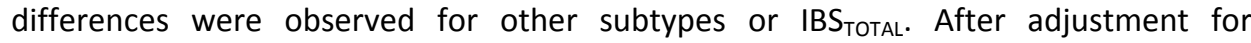
demographical factors, psychological symptoms and lifestyle, the L/R ratio remained significantly higher for IBS-D relative to $\mathrm{HC}(\beta: 0.53,95 \% \mathrm{Cl}: 0.03 ; 1.03, \mathrm{p}=0.037)$. This association was even slightly stronger when we additionally adjusted for use of medication ( $\beta$ : 0.63, 95\% Cl: 0.09; 1.16, $p=0.022$ ).

Separately adjusting the crude analysis for each factor revealed that only male sex was associated with increased $L / R$ ratio $(\beta: 0.31,95 \% \mathrm{Cl}:-0.01 ; 0.63, p=0.054)$, although not reaching statistical significance. When thereafter adjusting the crude analysis for all factors combined and excluding sex, the associations between IBS groups and L/R ratio was not affected (data not shown), demonstrating that the effect of the investigated confounders on the association between IBS and L/R ratio is small.

\section{Colon and whole gut permeability}

Linear regression analysis of S/E-ratio in the 5-24h of urine fraction, indicating colonic permeability, and in 0-24h urine, indicating whole-gut permeability, without (see also Table 2.2) or with adjustments for possible confounding factors (data not shown), did

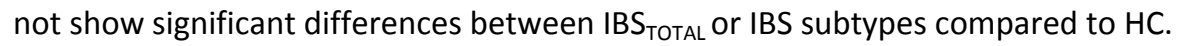

\section{Correlation between intestinal permeability and symptoms}

Symptoms of abdominal pain, diarrhea and indigestion syndrome as measured by the GSRS, showed a statistically significant but weak positive correlation with sucrose excretion (all Spearman's rho<0.27; $\mathrm{p}=0.001$ ). Diarrhea and indigestion syndrome also showed a significant but weak positive correlation with L/R ratio (all Spearman's rho<0.17; $\mathrm{p}<0.05)$. No significant correlation was found for other symptoms, nor between GI symptoms and colonic or whole gut permeability. Correlations between symptoms measured by the 14-days symptom diary and intestinal permeability were comparable (data not shown). 


\section{Discussion}

In this paper, we report on gut permeability testing, employing a validated multi-sugar test that simultaneously measures permeability at various sites in the GI tract, in a large and well-characterized IBS population and a healthy control group. In addition, we evaluated the effect of possible confounding factors. Sucrose excretion was significantly

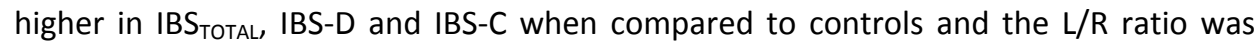
significantly higher in IBS-D patients. However, after adjustment for possible confounders, only the $L / R$ ratio, indicating small intestinal permeability, in IBS-D remained significantly increased when compared to controls.

We have used a validated multi-sugar test to analyse site-specific permeability of the intestine. The advantage of this test is that low dosages of 5 different sugars were given and analysed in two urine fractions, i.e. $0-5 h$ and 5-24h, as indicators for gastroduodenal, small intestinal, colonic and whole gut permeability (see also Figure 2.1). The site-specificity of the different sugars and ratios used is tested previously in animal and human studies. ${ }^{16-18,23-25}$ Sucrose (a disaccharide) is hydrolysed by the enzyme sucrase in the duodenum, ${ }^{23}$ and has been demonstrated to indicate gastroduodenal permeability when measured in 0-5h urine. Lactulose (a disaccharide, not broken down by human enzymes) can permeate by the paracellar route and rhamnose (a mono-saccharide) by the transcellular route. As both are degraded by bacteria that are most abundant in the colon, ${ }^{16}$ they are typically used as markers for small intestinal permeability. ${ }^{16-18,24,26,27}$ With respect to urine sampling after sugar intake, it is not yet clear whether $0-1 \mathrm{~h}, 0-2 \mathrm{~h}$ or $0-5 \mathrm{~h}$ urine fractions best reflect small intestinal permeability. Data from van Wijck et al. indicate however, that $0-5 \mathrm{~h}$ fractions in the applied multi-sugar test, do reflect small intestinal permeability. ${ }^{17,18}$ Sucralose (a disaccharide) and erythritol (a sugar alcohol) are both not broken down by human enzymes nor by human colonic bacteria. ${ }^{28,29}$ Therefore ratios of these sugars in 5-24h and $0-24 \mathrm{~h}$ urine are used as indicators for colonic and whole gut permeability, respectively. ${ }^{16-18,24}$ Permeability testing by sugar probes could possibly be affected by differences in gastric emptying and intestinal transit time, which were not assessed in our study. However, by using a total ingested sugar amount of only $4.5 \mathrm{~g}$ and the ratios of transcellularly and paracellularly absorbed sugar probes, these effects were minimized.

We found a significant difference in sucrose excretion, pointing to increased gastroduodenal permeability in IBS TOTAL $_{\text {as }}$ well as in IBS-C and IBS-D patients compared to HC. However, after adjusting for patient characteristics and psychological symptoms,

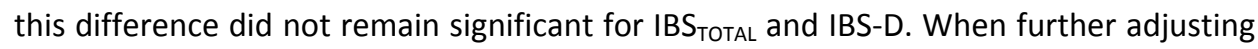
for lifestyle factors and use of medication the difference did not remain significant for 
IBS-C either, indicating that the observed difference was mainly based on confounding factors in our population.

Although IBS is a heterogeneous disease associated with a wide variety of patient characteristics and environmental factors (e.g. sex differences, psychosocial events, medication), most studies assessing intestinal permeability in IBS patients have not taken into account the possible confounding effects of these factors. Therefore it is not known whether their observations on permeability were associated with the disease per se or with the presence of possible confounders. Only two studies reported on a limited number of possible confounders. In the Walkerton cohort of post infectious IBS patients, male sex was found to be associated with increased lactulose-mannitol ratio in overnight urine fractions, ${ }^{8}$ which was previously demonstrated in healthy controls, ${ }^{30}$ pointing to a physiological sex difference. Furthermore NSAIDs were shown to compromise whole gut permeability in both IBS patients and healthy controls. ${ }^{12}$

We have analysed a large number of potential confounding factors. A higher BMI was shown to have an important confounding effect on the association between IBS and sucrose excretion in our study, which is in line with previously reported increased intestinal permeability in obese healthy subjects compared to lean. ${ }^{31}$ The most pronounced effect on sucrose excretion was found when we separately adjusted for the use of drugs that positively affect motility, i.e. prokinetics and laxatives. A possible mechanism by which prokinetics increase intestinal permeability could be the dopaminergic receptor stimulation by these drugs which in animal models was found to be associated with intestinal barrier alterations. ${ }^{32}$ One study postulated that laxatives increase intestinal permeability by direct effects on tight junction proteins. ${ }^{33}$

Male sex, smoking and use of PPI's were associated with higher sucrose excretion in our study, but did not have a confounding effect in our study population. In previous studies an increased sucrose excretion was found in patients with gastro-oesophageal reflux disease as well as in healthy controls that use PPI's. It was hypothesized that this may be caused by PPI-related effects on the intracellular potassium and calcium homeostasis, which could affect the cytoskeleton and thereby tight junctions between epithelial cells. ${ }^{34}$ We cannot fully rule out that PPIs or other medications did not have any causal effect on permeability, but rather that the underlying condition for which they were used were the cause of the observed association. However, we did not see differences in permeability on all GI sites between patients with and without reflux/dyspepsia symptoms, being conditions clearly associated with PPI use. With respect to smoking, previous reports showed no difference in intestinal permeability between smoking and non-smoking patients with inflammatory bowel disease and healthy subjects. ${ }^{35,36}$ With respect to use of NSAIDs, it has been observed that these drugs can increase intestinal permeability in IBS patients and healthy subjects shortly after intake, i.e. acute effects. ${ }^{12,27}$ We instructed study subjects to refrain from use of NSAIDs or alcohol in the two days prior and during the permeability test, in order to eliminate acute effect of these substances on intestinal permeability. Therefore, our 
data reflect chronic rather than acute effects and these factors did not reveal to be confounders in our population. In a post-hoc analysis we also tested the effect of alcohol intake versus no alcohol intake in general for our population, and no effect of alcohol intake on intestinal permeability was observed in this case as well (data not shown).

The urinary $L / R$ ratio was affected and points to an increased small intestinal permeability in patients with IBS-D compared to HC, but not in other IBS-subtypes. Our finding is in line with previous observations in small sized studies (12 to 19 IBS-D patients). ${ }^{5,7,11}$ The significant difference in L/R ratio between IBS-D and HC that we observed persisted after adjustment for confounders.

The increased permeability, reflecting intestinal barrier dysfunction of the small bowel, may represent an important pathophysiological mechanism in IBS-D. This finding is supported by recent mechanistic studies revealing not only an increased number and activation of mast cells in jejunal biopsies of IBS-D patients compared to HC, but also significant differences in global gene expression, particularly in tight-junction signaling pathways, $^{37}$ and structural alterations of the apical junction complex correlating positively with mast cell activation as well as with clinical symptoms. ${ }^{38}$ These data support the concept of IBS-D as a functional gut disorder or entity having specific organic substrates and underlying pathophysiological mechanisms.

Whether increased intestinal permeability is an early event or consequence of IBS remains to be determined. Previous findings indicate that barrier dysfunction may contribute to symptom generation in $\mathrm{IBS}^{10,38,39}$ and point to the direction of barrier dysfunction as an early event, facilitating permeation of $e . g$. bacteria and their products (i.e. lipopolysaccharides), resulting in immune activation ${ }^{40,41}$ which may further increase paracellular epithelial permeability and subsequent low-grade mucosal inflammation. ${ }^{42-44}$ Increased intestinal permeability has also been associated with visceral hypersensitivity in IBS patients. ${ }^{10}$ However, it has to be noted that increased intestinal permeability may be a consequence, rather than a causative factor of IBS. Recent studies have shown that acute stress and factors along the gut brain axis, e.g. serotonin $^{45}$ and corticotropin-releasing factor (CRF), ${ }^{46}$ modulate intestinal permeability by mast cell-dependent mechanisms in murine models as well as in healthy human subjects, postulating that the enteric and central nervous system may have a regulatory effect on the intestinal barrier function. ${ }^{46-49}$ Since this evidence exists for an association between acute stress and increased intestinal permeability in healthy individuals, we have analysed the influence of chronic symptoms of anxiety and depression on intestinal permeability (Model 2) in IBS patients. Symptoms of anxiety and depression appear to account for part of the increased sucrose excretion and L/R ratio in IBS patients; however, in the additional analysis of the separate co-factors the effect of psychological symptoms on intestinal permeability was only weak and not statistically significant. 
The identification of increased small intestinal permeability in patients with IBS-D may become relevant from a therapeutic perspective. However, first more mechanistic research is needed to provide a definition of a clinically relevant difference in intestinal permeability, e.g. associated to increased bacterial translocation or endotoxin levels and subsequent mucosal inflammation contributing to symptom generation in the target population.

Previous data on in vivo colonic permeability in IBS or IBS-D are contrasting, i.e. lower, ${ }^{6}$ higher $^{5,11,13}$ or unaltered colonic permeability ${ }^{7}$ when compared to $\mathrm{HC}$. There are however some indications of impaired barrier function in studies focussing on tight junctions in colonic biopsies from IBS patients, with reduced expression of zonula occludens-1 and occludin proteins. ${ }^{50}$ Previous reports on whole gut permeability do point to the direction of increased permeability in post-infectious IBS and IBS-D, 8,10 while no differences were found for IBS patients in general when compared to $\mathrm{HC}^{6,12}$ However, all studies used different methods to assess the colonic and whole gut permeability and included relatively small numbers of subjects or assessed permeability only in post-infectious IBS patients. Our aim was to clarify this point by assessing colonic as well as whole gut permeability in a large group of IBS patients, using the validated multi-sugar test. Our results however, do not show statistically significant differences in S/E ratio in 5-24h and $0-24 \mathrm{~h}$ urine fraction, indicating no alternations in colonic or whole gut permeability, respectively, in IBS TOTAL or IBS subtypes when compared to HC.

IBS is a very heterogeneous disorder that is associated with a multitude of patient characteristics and chronic or intermittent use of medication. Based on literature data, we anticipated that some of these factors would affect intestinal permeability. Our results demonstrate that especially sucrose excretion was affected by confounders, thereby altering the conclusion with respect to gastroduodenal permeability in IBS patients. Based on the present findings, especially BMI, smoking history, medication and sex should be taken into account as possible confounders in future permeability studies. Moreover, after adjustment of our analysis for possible confounding factors only small intestinal permeability (L/R ratio) in IBS-D patients remained significantly increased compared to $\mathrm{HC}$.

Our findings clearly show the relevance of adjustment for possible confounding factors, as well as site-specific permeability assessment and IBS subtype differentiation in future studies analysing intestinal permeability in IBS patients.

Although we assessed intestinal permeability in a large heterogeneous IBS population, and the range of our confidence intervals of our fully adjusted models were similar to the crude model, and while analyses in our IBS subpopulations were largely consistent

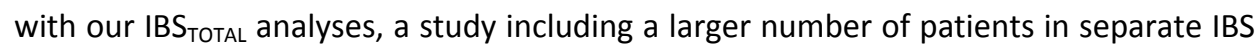


subtype groups would be warranted to confirm our findings in the subgroup analyses. Furthermore, it has to be noted that our results are based on cross-sectional and observational data and that causality was not studied.

\section{Conclusion}

By assessing intestinal permeability in a large group of IBS patients and healthy controls, we have demonstrated that $L / R$ ratio, representing small intestinal permeability is increased, irrespective of possible confounding factors, in patients with diarrhea predominant IBS when compared to healthy controls. This finding point to a different pathophysiological mechanism in this IBS subtype. Furthermore, we have shown that adjustment for possible confounders is essential when studying intestinal permeability, especially in a heterogeneous disorder as IBS. 


\section{References}

1. Simrén M, Brazier J, Coremans G, Dapoigny M, Müller-Lissner SA, Pace F, Smout AJ, Stockbrügger RW, Vatn $\mathrm{MH}$, Whorwell PJ. Quality of life and illness costs in irritable bowel syndrome. Digestion. 2004;69:254-61.

2. Spiegel BM. The burden of IBS: looking at metrics. Current gastroenterology reports. 2009;11:265-9.

3. Longstreth GF, Thompson WG, Chey WD, Houghton LA, Mearin F, Spiller RC. Functional bowel disorders. Gastroenterology. 2006;130:1480-91.

4. Camilleri M. Peripheral mechanisms in irritable bowel syndrome. N Engl J Med. 2012;367:1626-35.

5. Rao AS, Camilleri M, Eckert DJ, Busciglio I, Burton DD, Ryks M, Wong BS, Lamsam J, Singh R, Zinsmeister AR. Urine sugars for in vivo gut permeability: validation and comparisons in irritable bowel syndromediarrhea and controls. Am J Physiol Gastrointest Liver Physiol. 2011;301:G919-28.

6. Del Valle-Pinero AY, Van Deventer HE, Fourie NH, Martino AC, Patel NS, Remaley AT, Henderson WA. Gastrointestinal permeability in patients with irritable bowel syndrome assessed using a four probe permeability solution. Clin Chim Acta. 2013;418:97-101.

7. Dunlop SP, Hebden J, Campbell E, Naesdal J, Olbe L, Perkins AC, Spiller RC. Abnormal intestinal permeability in subgroups of diarrhea-predominant irritable bowel syndromes. Am J Gastroenterol. 2006;101:1288-94.

8. Marshall JK, Thabane M, Garg AX, Clark W, Meddings J, Collins SM; WEL Investigators. Intestinal permeability in patients with irritable bowel syndrome after a waterborne outbreak of acute gastroenteritis in Walkerton, Ontario. Aliment Pharmacol Ther. 2004;20:1317-22.

9. Spiller RC, Jenkins D, Thornley JP, Hebden JM, Wright T, Skinner M, Neal KR. Increased rectal mucosal enteroendocrine cells, $\mathrm{T}$ lymphocytes, and increased gut permeability following acute Campylobacter enteritis and in post-dysenteric irritable bowel syndrome. Gut. 2000;47:804-11.

10. Zhou Q, Zhang B, Verne GN. Intestinal membrane permeability and hypersensitivity in the irritable bowel syndrome. Pain. 2009;146:41-6.

11. Zhou Q, Souba WW, Croce CM, Verne GN. MicroRNA-29a regulates intestinal membrane permeability in patients with irritable bowel syndrome. Gut. 2010;59:775-84.

12. Kerckhoffs AP, Akkermans LM, de Smet MB, Besselink MG, Hietbrink F, Bartelink IH, Busschers WB, Samsom M, Renooij W. Intestinal permeability in irritable bowel syndrome patients: effects of NSAIDs. Dig Dis Sci. 2010;55:716-23.

13. Gecse K1, Róka R, Séra T, Rosztóczy A, Annaházi A, Izbéki F, Nagy F, Molnár T, Szepes Z, Pávics L, Bueno $\mathrm{L}$, Wittmann T. Leaky gut in patients with diarrhea-predominant irritable bowel syndrome and inactive ulcerative colitis. Digestion. 2012;85:40-6.

14. Park JH, Park DI, Kim HJ, Cho YK, Sohn Cl, Jeon WK, Kim BI, Won KH, Park SM. The Relationship between Small-Intestinal Bacterial Overgrowth and Intestinal Permeability in Patients with Irritable Bowel Syndrome. Gut Liver. 2009;3:174-9.

15. Ersryd A, Posserud I, Abrahamsson H, Simren M. Subtyping the irritable bowel syndrome by predominant bowel habit: Rome II versus Rome III. Aliment Pharmacol Ther. 2007;26:953-61.

16. Meddings JB, Gibbons I. Discrimination of site-specific alterations in gastrointestinal permeability in the rat. Gastroenterology. 1998;114:83-92.

17. van Wijck K, van Eijk HM, Buurman WA, Dejong $\mathrm{CH}$, Lenaerts K. Novel analytical approach to a multisugar whole gut permeability assay. Journal of chromatography B, Analytical technologies in the biomedical and life sciences. 2011;879:2794-801.

18. van Wijck K, Verlinden TJ, van Eijk HM, Dekker J, Buurman WA, Dejong CH, Lenaerts K. Novel multisugar assay for site-specific gastrointestinal permeability analysis: a randomized controlled crossover trial. Clin Nutr. 2013;32:245-51.

19. Svedlund J, Sjodin I, Dotevall G. GSRS--a clinical rating scale for gastrointestinal symptoms in patients with irritable bowel syndrome and peptic ulcer disease. Dig Dis Sci. 1988;33:129-34.

20. Thijssen AY, Jonkers DM, Leue C, van der Veek PP, Vidakovic-Vukic M, van Rood YR, Clemens $\mathrm{CH}$, Masclee AA. Dysfunctional cognitions, anxiety and depression in irritable bowel syndrome. J Clin Gastroenterol. 2010;44:e236-41. 
21. Zigmond AS, Snaith RP. The hospital anxiety and depression scale. Acta Psychiatr Scand. 1983;67: 361-70.

22. Bjelland I, Dahl AA, Haug TT, Neckelmann D. The validity of the Hospital Anxiety and Depression Scale. An updated literature review. J Psychosom Res. 2002;52:69-77.

23. Sutherland LR, Verhoef M, Wallace JL, Van Rosendaal G, Crutcher R, Meddings JB. A simple, noninvasive marker of gastric damage: sucrose permeability. Lancet. 1994;343:998-1000.

24. Pijls KE, Koek GH, Elamin EE, de Vries H, Masclee AA, Jonkers DM. Large intestine permeability is increased in patients with compensated liver cirrhosis. Am J Physiol Gastrointest Liver Physiol. 2014;306:G147-53.

25. Rutten EP, Lenaerts K, Buurman WA, Wouters EF. Disturbed intestinal integrity in patients with COPD; effects of activities of daily living. Chest. 2013 Aug 8.

26. Lambert GP, Schmidt A, Schwarzkopf K, Lanspa S. Effect of aspirin dose on gastrointestinal permeability. Int J Sports Med. 2012;33:421-5.

27. Troost FJ, Saris WH, Brummer RJ. Recombinant human lactoferrin ingestion attenuates indomethacininduced enteropathy in vivo in healthy volunteers. Eur J Clin Nutr. 2003;57:1579-85.

28. Roberts A, Renwick AG, Sims J, Snodin DJ. Sucralose metabolism and pharmacokinetics in man. Food Chem Toxicol. 2000;38 Suppl 2:S31-41.

29. Arrigoni E, Brouns F, Amado R. Human gut microbiota does not ferment erythritol. Br J Nutr. 2005;94:643-6.

30. Suenaert P, Bulteel V, Den Hond E, Hiele M, Peeters M, Monsuur F, Ghoos Y, Rutgeerts P. The effects of smoking and indomethacin on small intestinal permeability. Aliment Pharmacol Ther. 2000;14:819-22.

31. Teixeira TF, Souza NC, Chiarello PG, Franceschini SC, Bressan J, Ferreira CL, Peluzio Mdo C. Intestinal permeability parameters in obese patients are correlated with metabolic syndrome risk factors. Clin Nutr. 2012;31:735-40.

32. Zhang $X$, Jiang $X$. Effects of enteral nutrition on the barrier function of the intestinal mucosa and dopamine receptor expression in rats with traumatic brain injury. JPEN J Parenter Enteral Nutr. 2015;39:114-23.

33. Rummel W. Biologic membrane functions in health and disease. Effects of bile acids and laxatives on the mucosal transfer. Bull Schweiz Akad Med Wiss. 1976;32:233-50.

34. Mullin JM, Valenzano MC, Whitby M, Lurie D, Schmidt JD, Jain V, et al. Esomeprazole induces upper gastrointestinal tract transmucosal permeability increase. Aliment Pharmacol Ther. 2008;28:1317-25.

35. Gotteland M, Cruchet S, Frau V, Wegner ME, Lopez R, Herrera T, Sanchez A, Urrutia C, Brunser O. Effect of acute cigarette smoking, alone or with alcohol, on gastric barrier function in healthy volunteers. Dig Liver Dis. 2002;34:702-6.

36. Benoni $\mathrm{C}$, Prytz $\mathrm{H}$. Effects of smoking on the urine excretion of oral 51Cr EDTA in ulcerative colitis. Gut. 1998;42:656-8.

37. Martinez C, Gonzalez-Castro A, Vicario M, Santos J. Cellular and molecular basis of intestinal barrier dysfunction in the irritable bowel syndrome. Gut Liver. 2012;6:305-15.

38. Martínez C, Lobo B, Pigrau M, Ramos L, González-Castro AM, Alonso C, Guilarte M, Guilá M, de Torres I, Azpiroz F, Santos J, Vicario M. Diarrhoea-predominant irritable bowel syndrome: an organic disorder with structural abnormalities in the jejunal epithelial barrier. Gut. 2013;62:1160-8.

39. Barbara G, Stanghellini V, De Giorgio R, Cremon C, Cottrell GS, Santini D, Pasquinelli G, Morselli-Labate AM, Grady EF, Bunnett NW, Collins SM, Corinaldesi R. Activated mast cells in proximity to colonic nerves correlate with abdominal pain in irritable bowel syndrome. Gastroenterology. 2004;126: 693-702.

40. Turner JR. Intestinal mucosal barrier function in health and disease. Nat Rev Immunol. 2009;9:799-809.

41. Belmonte L, Beutheu Youmba S, Bertiaux-Vandaële N, Antonietti M, Lecleire S, Zalar A, Gourcerol G, Leroi $A M$, Déchelotte $P$, Coëffier $M$, Ducrotté $P$. Role of toll like receptors in irritable bowel syndrome: differential mucosal immune activation according to the disease subtype. PloS One. 2012;7:e42777.

42. Camilleri M, Madsen K, Spiller R, Greenwood-Van Meerveld B, Verne GN. Intestinal barrier function in health and gastrointestinal disease. Neurogastroenterol Motil. 2012;24:503-12.

43. Gwee KA, Collins SM, Read NW, Rajnakova A, Deng Y, Graham JC, McKendrick MW, Moochhala SM. Increased rectal mucosal expression of interleukin 1beta in recently acquired post-infectious irritable bowel syndrome. Gut. 2003;52:523-6. 
44. Chadwick VS, Chen W, Shu D, Paulus B, Bethwaite P, Tie A, Wilson I. Activation of the mucosal immune system in irritable bowel syndrome. Gastroenterology. 2002;122:1778-83.

45. Keszthelyi D. The serotonin precursor 5-hydroxytryptophan reinforces intestinal barrier function [abstract]. . Gastroenterology. 2011 2011;140:S370.

46. Vanuytsel $T$, van Wanrooy S, Vanheel H, Vanormelingen $C$, Verschueren S, Houben E, Salim Rasoel S, Tóth J, Holvoet L, Farré R, Van Oudenhove L, Boeckxstaens G, Verbeke K, Tack J. Psychological stress and corticotropin-releasing hormone increase intestinal permeability in humans by a mast celldependent mechanism. Gut. 2014;63:1293-9

47. Barreau F, Cartier C, Leveque M, Ferrier L, Moriez R, Laroute V, Rosztoczy A, Fioramonti J, Bueno L. Pathways involved in gut mucosal barrier dysfunction induced in adult rats by maternal deprivation: corticotrophin-releasing factor and nerve growth factor interplay. J Physiol. 2007;580:347-56.

48. Demaude J, Salvador-Cartier C, Fioramonti J, Ferrier L, Bueno L. Phenotypic changes in colonocytes following acute stress or activation of mast cells in mice: implications for delayed epithelial barrier dysfunction. Gut. 2006;55:655-61.

49. Snoek SA, Verstege MI, Boeckxstaens GE, van den Wijngaard RM, de Jonge WJ. The enteric nervous system as a regulator of intestinal epithelial barrier function in health and disease. Exp Rev Gastroenterol Hepatol. 2010;4:637-51.

50. Bertiaux-Vandaële N, Youmba SB, Belmonte L, Lecleire S, Antonietti M, Gourcerol G, Leroi AM, Déchelotte $P$, Ménard JF, Ducrotté P, Coëffier $M$. The expression and the cellular distribution of the tight junction proteins are altered in irritable bowel syndrome patients with differences according to the disease subtype. Am J Gastroenterol. 2011;106:2165-73. 


\section{Supplemental data}

Table S2.1 Permeability test: sugar excretion and ratios of excreted sugars as measured in urine (in 0-5, 5-24 and 0-24 hours fraction), for HC, IBS

\begin{tabular}{|c|c|c|c|c|c|}
\hline Sugar excretion & $\begin{array}{c}\mathrm{HC} \\
(n=94)\end{array}$ & $\begin{array}{l}\mathrm{IBS}_{\text {TOTAL }} \\
(\mathrm{n}=91)\end{array}$ & $\begin{array}{l}\text { IBS-D } \\
(n=34)\end{array}$ & $\begin{array}{c}\text { IBS-C } \\
(n=21)\end{array}$ & $\begin{array}{l}\text { IBS-M } \\
(n=30)\end{array}$ \\
\hline $\begin{array}{l}0-5 \mathrm{~h} \text { Sucrose } \\
(\mu \mathrm{mol})\end{array}$ & $\begin{array}{c}2.44 \\
{[0.91 ; 5.85]}\end{array}$ & $\begin{array}{c}5.26 \\
{[1.82 ; 11.03]^{\mathrm{b}}}\end{array}$ & $\begin{array}{c}4.22 \\
{[2.12 ; 8.03]^{\mathrm{a}}}\end{array}$ & $\begin{array}{c}7.40 \\
{[2.37 ; 18.29]^{b}}\end{array}$ & $\begin{array}{c}6.59 \\
{[1.08 ; 12.13]}\end{array}$ \\
\hline $0-5 \mathrm{~h} \mathrm{~L} / \mathrm{R}$ ratio & $\begin{array}{c}0.014 \\
{[0.008 ; 0.025]}\end{array}$ & $\begin{array}{c}0.018 \\
{[0.011 ; 0.029]}\end{array}$ & $\begin{array}{c}0.023 \\
{[0.013 ; 0.038]^{\mathrm{a}}}\end{array}$ & $\begin{array}{c}0.018 \\
{[0.009 ; 0.032]}\end{array}$ & $\begin{array}{c}0.016 \\
{[0.011 ; 0.024]}\end{array}$ \\
\hline 5-24h S/E ratio & $\begin{array}{c}0.010 \\
{[0.006 ; 0.025]}\end{array}$ & $\begin{array}{c}0.008 \\
{[0.004 ; 0.017]}\end{array}$ & $\begin{array}{c}0.008 \\
{[0.004 ; 0.020]}\end{array}$ & $\begin{array}{c}0.009 \\
{[0.003 ; 0.015]}\end{array}$ & $\begin{array}{c}0.008 \\
{[0.006 ; 0.024]}\end{array}$ \\
\hline $0-24 \mathrm{~h}$ S/E ratio & $\begin{array}{c}0.009 \\
{[0.006 ; 0.013]}\end{array}$ & $\begin{array}{c}0.009 \\
{[0.006 ; 0.014]}\end{array}$ & $\begin{array}{c}0.009 \\
{[0.004 ; 0.014]}\end{array}$ & $\begin{array}{c}0.008 \\
{[0.004 ; 0.014]}\end{array}$ & $\begin{array}{c}0.010 \\
{[0.006 ; 0.016]}\end{array}$ \\
\hline $\begin{array}{l}\text { 0-5h Lactulose } \\
(\mu \mathrm{mol})\end{array}$ & $\begin{array}{c}5.16 \\
{[2.17 ; 9.92]}\end{array}$ & $\begin{array}{c}5.57 \\
{[3.18 ; 9.51]}\end{array}$ & $\begin{array}{c}7.19 \\
{[4.04 ; 10.41]}\end{array}$ & $\begin{array}{c}5.81 \\
{[1.26 ; 10.42]}\end{array}$ & $\begin{array}{c}5.44 \\
{[3.24 ; 8.39]}\end{array}$ \\
\hline $\begin{array}{l}\text { 0-5h Rhamnose } \\
(\mu \mathrm{mol})\end{array}$ & $\begin{array}{c}318.28 \\
{[224.51 ; 520.75]}\end{array}$ & $\begin{array}{c}314.24 \\
{[222.45 ; 409.20]}\end{array}$ & $\begin{array}{c}306.81 \\
{[219.83 ; 393.74]}\end{array}$ & $\begin{array}{c}251.88 \\
{[174.07 ; 429.60]}\end{array}$ & $\begin{array}{c}314.88 \\
{[241.39 ; 424.57]}\end{array}$ \\
\hline $\begin{array}{l}5-24 \mathrm{~h} \text { Sucralose } \\
(\mu \mathrm{mol})\end{array}$ & $\begin{array}{c}26.23 \\
{[12.10 ; 44.30]}\end{array}$ & $\begin{array}{c}20.63 \\
{[6.68 ; 37.38]}\end{array}$ & $\begin{array}{c}21.20 \\
{[6.4 ; 39.02]}\end{array}$ & $\begin{array}{c}15.65 \\
{[6.40 ; 35.74]}\end{array}$ & $\begin{array}{c}17.75 \\
{[7.12 ; 41.79]}\end{array}$ \\
\hline $\begin{array}{l}5-24 \mathrm{~h} \text { Erythritol } \\
(\mu \mathrm{mol})\end{array}$ & $\begin{array}{c}2588.31 \\
{[1486.48 ; 3438.62]}\end{array}$ & $\begin{array}{c}2411.21 \\
{[1625.79 ; 3259.83]}\end{array}$ & $\begin{array}{c}2509.52 \\
{[1674.39 ; 3303.76]}\end{array}$ & $\begin{array}{c}2553.81 \\
{[1727.37 ; 3355.94]}\end{array}$ & $\begin{array}{c}2153.04 \\
{[1359.13 ; 3088.66]}\end{array}$ \\
\hline $\begin{array}{l}0-24 \mathrm{~h} \text { Sucralose } \\
(\mu \mathrm{mol})\end{array}$ & $\begin{array}{c}44.14 \\
{[27.46 ; 66.81]}\end{array}$ & $\begin{array}{c}40.78 \\
{[24.32 ; 67.32]}\end{array}$ & $\begin{array}{c}39.82 \\
{[22.06 ; 68.97]}\end{array}$ & $\begin{array}{c}36.73 \\
{[18.85 ; 62.21]}\end{array}$ & $\begin{array}{c}44.52 \\
{[35.81 ; 73.81]}\end{array}$ \\
\hline $\begin{array}{l}\text { 0-24h Erythritol } \\
(\mu \mathrm{mol})\end{array}$ & $\begin{array}{c}4426.39 \\
{[3257.37 ; 5732.76]}\end{array}$ & $\begin{array}{c}4279.75 \\
{[3556.06 ; 4931.84]}\end{array}$ & $\begin{array}{c}4281.13 \\
{[3584.19 ; 4916.00]}\end{array}$ & $\begin{array}{c}4379.13 \\
{[3529.42 ; 5152.75]}\end{array}$ & $\begin{array}{c}4190.25 \\
{[3557.68 ; 5220.79]}\end{array}$ \\
\hline
\end{tabular}

Results, not adjusted for confounders, presented as: median [interquartile range (IQR), i.e. Q1; Q3]. Differences, relative to $\mathrm{HC}$, tested with linear regression. Skewed variables were $\mathrm{LN}$ transformed prior to analysis. ${ }^{a} \mathrm{p}<0.05,{ }^{b} \mathrm{p}<0.01$ vs. $\mathrm{HC}$ 


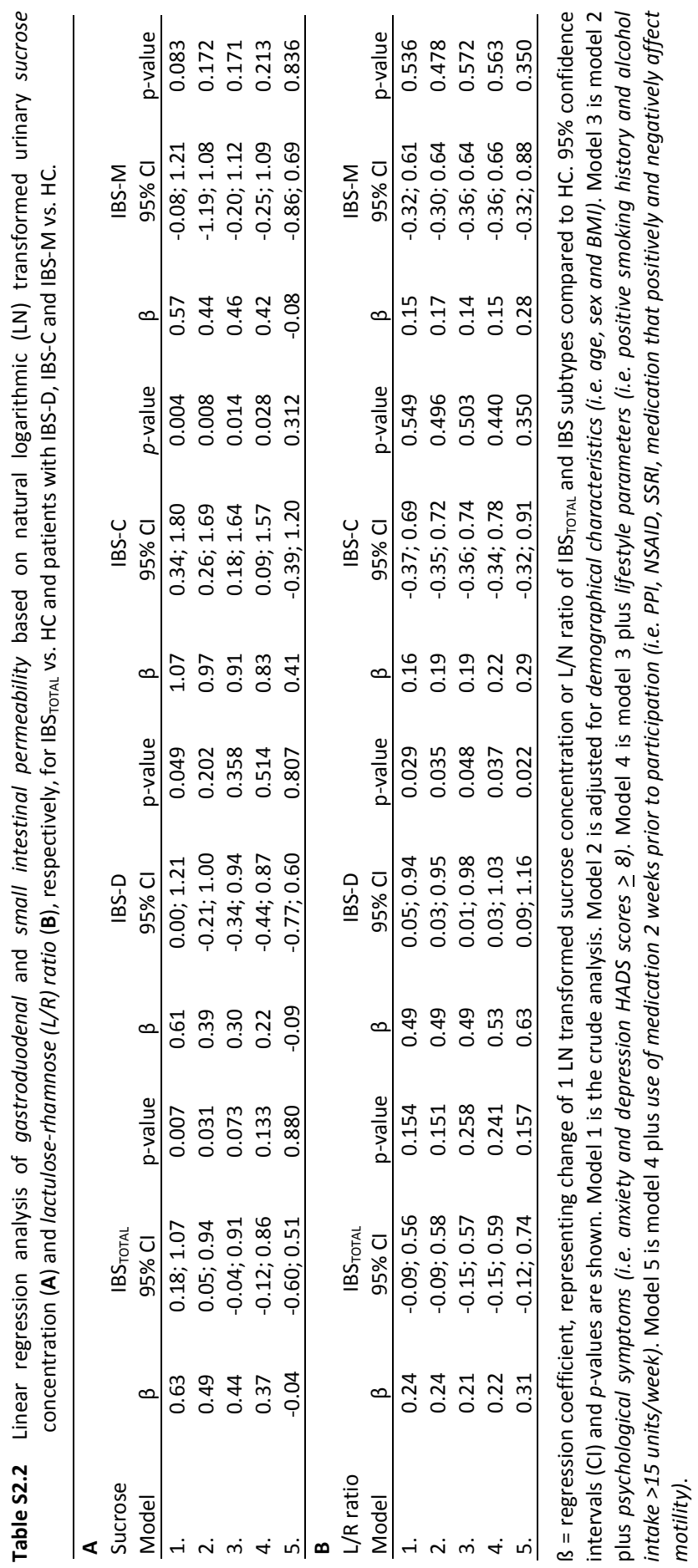


IBS and intestinal permeability 


\section{Chapter}

Effect of L. plantarum strains on intestinal mucosa

The effects of oral administration of three Lactobacillus plantarum strains on intestinal barrier function and gene transcription in human small intestinal mucosa; a randomized double-blind placebo controlled trial

Zlatan Mujagic, Paul de Vos, Mark V Boekschoten, Coen Govers, Harm-Jan HM Pieters, Nicole JW de Wit, Peter A Bron, Ad AM Masclee, Freddy J Troost

\section{Submitted}




\section{Abstract}

\section{Background and aims}

The aim of this study was to investigate the effects of three Lactobacillus plantarum strains on in vivo small intestinal barrier function and gene transcription in healthy subjects. The strains were selected for their differential effects on TLR signalling and the organization of the epithelial tight junctions, which may lead to beneficial effects in a stressed gut mucosa.

\section{Methods}

Ten healthy volunteers participated in four different intervention periods: 7-day oral intake of either L. plantarum WCFS1, CIP104448, TIFN101 or placebo, proceeded by a 4 weeks wash-out period. Indomethacin, a NSIAD, was used to stress small intestinal barrier function. The ratio in 5-hr urinary recovery of orally ingested lactulose and rhamnose indicated small intestinal permeability. Duodenal mucosal biopsies were obtained by gastroduodenoscopy for tight-junction, and host genome-wide microarray (Affymetrix Human Gene 1.1ST) analyses.

\section{Results}

Lactulose-rhamnose ratio increased after intake of indomethacin (mean ratio $0.06 \pm 0.04$ to $0.10 \pm 0.06, p=0.001$ ), but was not significantly affected by the interventions. However, gene transcription pathway analysis demonstrated that particularly L. plantarum TIFN101 modulated cell-cell adhesion with high turnover of genes involved in tight- and adhesion junction protein synthesis and degradation (e.g. actinin alpha-4, metalloproteinase-2). These effects were less pronounced for L. plantarum WCFS1 and CIP104448.

\section{Conclusion}

Indomethacin induced an increase in small intestinal permeability, not affected by any of the bacterial strain interventions. However, intake of the bacterial strains did induce strain specific effects on gene transcription in mucosal cells. L. plantarum TIFN101 induced the most pronounced probiotic properties with specific effects on repair processes in the compromised intestine of healthy subjects. 


\section{Introduction}

Impairment of intestinal barrier function has been implicated as an early event in the pathogenesis of various intestinal and systemic disorders. It may lead to increased permeation of substances present in the gut lumen, such as bacteria and their products (e.g., lipopolysaccharides), into the mucosal layer and may result in local and systemic inflammatory responses. ${ }^{1-3}$ This process is assumed to play a role in the pathophysiology of organic gastro-intestinal (GI) disorders such as inflammatory bowel diseases, ${ }^{4,5}$ and celiac disease, ${ }^{6}$ but also with functional Gl disorders, such as irritable bowel syndrome. ${ }^{7,8}$ Furthermore, it has been associated with systemic disorders, such as diabetes mellitus, ${ }^{9}$ atopic eczema, ${ }^{10}$ liver disease, ${ }^{11}$ as well as the use of medication, such as non-steroidal anti-inflammatory drugs (NSAIDs). ${ }^{12,13}$ NSAIDs are frequently consumed worldwide but have also been used in research as a model to stress the intestinal mucosal layer and barrier function in healthy subjects. ${ }^{14}$ Up to now, no therapeutic agents have been developed that are able to successfully restore the intestinal mucosal barrier and thereby influence disease outcome. ${ }^{15}$ Positive effects on intestinal barrier function have been attributed to probiotic bacteria. ${ }^{16-21}$ However, further research is needed to prove these beneficial effects in humans and to gain further insight into the mechanisms through which live bacterial organisms improve the human gut barrier function. ${ }^{22}$

In a healthy intestinal homeostasis commensal non-pathogenic bacteria interact with host mucosa and thereby presumably co-regulate mucosal barrier function. Lactobacillus plantarum, a commensal bacterium present in humans, has been reported to reinforce the intestinal barrier and to reduce intestinal permeability in animal studies. ${ }^{17-19}$ In a previous study by our consortium, the administration of $L$. plantarum WCFS1 directly into the duodenum of healthy human subjects beneficially contributed to the organization of the epithelial tight junctions. ${ }^{16}$ Next to L. plantarum WCFS1, we have selected two more L. plantarum strains, i.e. CIP104448 (CIP48), and TIFN101 (in previous studies referred to as CIP104450), to be tested in the current study. They were selected based on research wherein these bacterial strains were shown to have differential effects in toll-like receptor (TLR) signalling in vitro and to affect both the innate and specific immune system both in vitro and in vivo. ${ }^{23-25}$ Although it has recently been demonstrated in an in vitro model that TLR signalling is involved in regulation of the intestinal barrier function, ${ }^{26}$ it is unknown whether these three $L$. plantarum strains have positive effects on intestinal mucosal barrier in human subjects after ingestion, or through which mechanisms these live bacterial organisms may improve the human gut barrier function.

We hypothesized that daily oral administration of L. plantarum WCFS1, CIP48, and TIFN101 over a 7-day period (i) will have positive effects on compromised (due to 
intake of NSAIDs) intestinal barrier function in healthy human subjects, with regard to intestinal permeability, tight junction protein- and gene expression, and furthermore (ii) will induce alterations in the transcription of pathways involved in mucosal structure and cell function, as measured in duodenal biopsy material.

Aim of the present randomized, double-blind, placebo-controlled trial was to assess the effects of oral intake of the three $L$. plantarum strains over a 7-day period on small intestinal permeability, duodenal epithelial tight junction protein expression and mucosal gene transcription, in healthy human subjects.

\section{Materials and methods}

The study protocol has been approved by the Maastricht University Medical Center+ Committee of Ethics, was in compliance with the Declaration of Helsinki (64th WMA General Assembly, Fortaleza, Brazil, 2013), and has been registered in the US National Library of Medicine (http://www.clinicaltrials.gov, NCT01456767).

\section{Study participants}

Healthy subjects were enrolled via public advertising, and were screened, involving a standardized general physical examination. All study participants gave written informed consent prior to inclusion. Exclusion criteria were current Gl symptoms, a history of severe disease or major abdominal surgery, use of any medication within 14 days or investigational drugs within 180 days prior to inclusion, blood donation within 3 months before the study period, pregnancy, lactation, obesity, smoking, use of drugs or excessive alcohol consumption, and known allergy/hypersensitivity towards intake of NSAIDs, sweeteners or pre- or probiotic supplements of any kind.

\section{Bacterial growth conditions and definition of placebo}

The bacterial strains L. plantarum WCFS1, ${ }^{27,28}$ CIP48, and TIFN101 ${ }^{23,24}$ were cultured overnight at $37^{\circ} \mathrm{C}$ in Man, Rogosa and Sharpe (MRS) medium (Merck, New Jersey, USA), to obtain stationary-phase cultures. Maltodextrin and glucose were added to a final concentration of $20 \%$ and $2 \%$ (wt/vol), respectively, to obtain bacterial preparations. Detailed protocols for culturing, harvesting, freeze-drying, storing, and viable count determining of Lactobacillus species have been published previously. ${ }^{29}$ Placebo controls only contained maltodextrin and glucose at similar final concentrations as bacterial supplements, ensuring a similar appearance and taste. Encoded and non-transparent vials containing the supplements were provided by NIZO Food Research (Ede, NL) to ensure the double-blind design. 


\section{Study design}

This human study was designed and conducted as a randomized double-blind placebocontrolled cross-over trial. The effects of three potentially beneficial bacterial strains were investigated in each subject during four randomized test periods (Supplementary Table S3.1). During each test period (Figure 3.1), one of the following supplements has been orally ingested twice a day, during breakfast and dinner, for 7 consecutive days: L. plantarum WCFS1 $\left(2.6 \times 10^{9}\right.$ colony forming units (cfu) per time point), L. plantarum CIP48 $\left(2.4 \times 10^{9} \mathrm{cfu} /\right.$ time point), L. plantarum TIFN101 $\left(5.9 \times 10^{9} \mathrm{cfu} /\right.$ time point) or placebo. Each test period was followed by a washout period of four weeks. There was no standardised diet for the study participants; however, subjects were instructed to consume on the last day of each test period the same diet as they did on the last day of the first period.

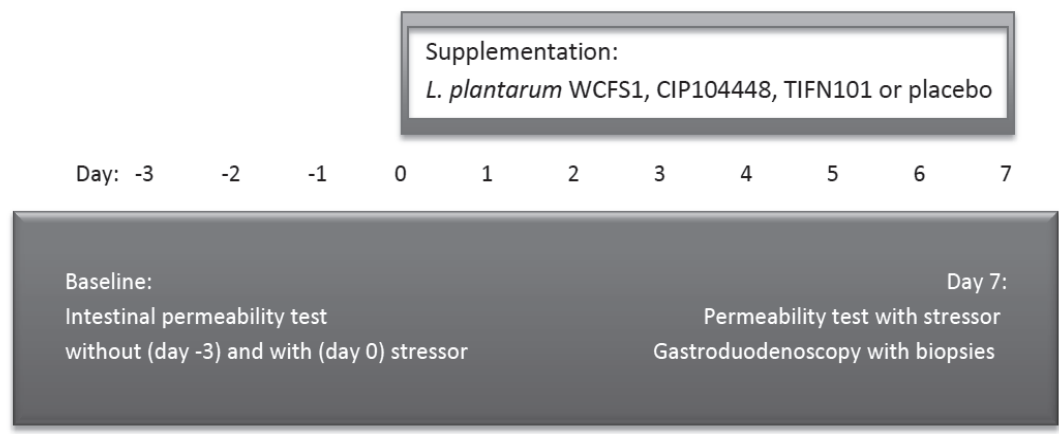

Figure 3.1 Schematic representation of a test period. Three and one-day prior to start of supplementation period, small intestinal permeability (L/R ratio) was assessed; at day -3 without, and at day 0 with indomethacin induced mucosal stress. Subsequently, a 7-day supplementation period followed, with oral intake of placebo or one of the three L. plantarum strains twice a day. At day 7 the permeability test with intake of indomethacin was repeated and biopsies were obtained using flexible gastroduodenoscopy.

Baseline permeability measurements were performed three days and one day prior to the intake of the supplements, in the absence and presence of the intestinal mucosal barrier stressor indomethacin, respectively. Subsequently, subjects consumed one of the three L. plantarum strains or placebo for seven consecutive days. Thereafter, the permeability test with addition of indomethacin (but not the test without indomethacin) was repeated, whereupon the same day standard flexible gastroduodenoscopy was performed to obtain six tissue samples from the duodenum (D2 section), at approximately $15 \mathrm{~cm}$ distal to the pylorus. The mucosal biopsies were immediately fresh frozen and used for RNA isolation followed by genome-wide microarray, and tight junction protein analysis. In line with previous research ${ }^{16,25,30}$ in 
which the effects of $L$. plantarum strains on intestinal mucosa were investigated, we focussed on the proximal small bowel, with minimal interference of other microbial interaction. This region of the small intestine is relatively accessible by means of endoscopy.

Possible side effects of the ingestion of the bacterial strains, adverse reactions to intake of indomethacin, and overall feelings of well-being were assessed using the validated gastrointestinal symptom rating scale $(\mathrm{GRSR})^{31}$ pre and post test period and during the intervention by a daily symptom diary. ${ }^{8}$

\section{In vivo small intestinal permeability test}

After an overnight fast, participants emptied their urinary bladder and consumed a drink (150 $\mathrm{ml}$ of tap water) containing $1 \mathrm{~g}$ lactulose (Centrafarm, Etten-Leur, the Netherlands) and $0.5 \mathrm{~g}$ L-rhamnose (Danisco, Copenhagen, Denmark). Participants were requested to remain fasted, with the exception of water intake, and to collect urine for 5 hours. Lactulose (disaccharide) can permeate the intestinal epithelial layer through the paracellular route and rhamnose (mono-saccharide) through the transcellular route. The ratio in $0-5 \mathrm{~h}$ urine (L/ $\mathrm{R}$ ratio) represents small intestinal permeability, and is used to minimize the effect of differences in gut motility and kidney function on the results. ${ }^{32}$ During and two days prior to the test period, excessive physical exercise and consumption of alcohol were prohibited for the study participants. Three days after this baseline permeability test and after the 7-day intervention period (Figure 3.1), the test was repeated with ingestion of indomethacin, a NSAID, administered in two different dosages; i.e. $75 \mathrm{mg}$ exactly 9 hours prior and $50 \mathrm{mg} 1$ hour prior to the intake of the sugars. ${ }^{13}$ Levels of the urinary excreted sugar probes were measured under blinded conditions by isocratic ion-exchange high-pressure liquid chromatography with mass spectrometry (HPLC-MS) as described previously. ${ }^{32}$

\section{RNA isolation, microarray processing and pathway analysis}

Total RNA was isolated from snap frozen (in liquid nitrogen) duodenal biopsies, using $1 \mathrm{ml}$ Trizol reagent (Invitrogen, Breda, NL). Subsequently it was purified using Qiagen RNeasy Micro kit (Qiagen, Venlo, NL). NanoDrop ND-1000 spectrophotometer (Isogen Life Science, De Meer, NL) was used for RNA quantification, and quality was checked by the Agilent 2100 bioanalyzer (Agilent Technologies, Amsterdam, NL). Samples were used for further analyses in case of intact bands corresponding to $18 \mathrm{~S}$ and $28 \mathrm{~S}$ ribosomal subunits and in the absence of chromosomal peaks or RNA degradation products. Whole transcript cDNA synthesis of total RNA (100 ng) material was conducted using Ambion WT expression kit (Life Technologies, Bleiswijk, NL), and was labeled using the Affymetrix GeneChip WT Terminal Labelling Kit (Affymetrix, Santa Clara, CA). Next, samples were hybridized to human whole genome Affymetrix GeneChip Human Gene 1.1 ST arrays, washed, stained, and scanned on an Affymetrix 
GeneTitan instrument, according to the User Guide for Expression Array Plates (P/N 702933 Rev.2).

Changes in gene expression were calculated as fold changes between placebo and the different $L$. plantarum strains by applying MADMAX(33) for statistical comparison analyses for multiple omics experiments. Functional interpretation of gene signatures (pairwise IBMT $p$-value <0.05) was performed using canonical pathway analysis of Ingenuity Pathway Analysis (IPA) 3.0 (Qiagen Ingenuity Systems, Redwood City, CA). Canonical pathways demonstrating significant differential regulation (threshold $p<0.05$ ) were considered for analysis.

Furthermore, gene transcription of tight junction proteins, i.e. claudin 3, claudin 4, myosin light chain kinase (MLCK), zonula occludens 1 (ZO-1) and occludin, in duodenal mucosal biopsies was also evaluated by RT-qPCR, as described previously(34), to confirm the microarray results for the transcription of these genes.

\section{Expression of tight junction proteins in duodenal mucosa samples}

Immunofluorescent staining for ZO-1 and occludin was performed on tissue-tek embedded frozen sections of the duodenal mucosa biopsy specimens, as described elsewhere. ${ }^{35}$ Leica TCS SPE confocal laser-scanning microscope equipped with a 50-mW Argon laser and a 1-mW HeNe laser (Leica Microsystems, Wetzlar, Germany), was used to examine the slides, and Image J software to process and analyze the confocal images, as described previously. ${ }^{36}$

\section{Data and statistical analysis}

Statistical analysis was performed using IBM SPSS Statistics version 22.0 (IBM Statistics for Windows, Armonk, NY). To compare all baseline L/R ratio measurements without and with indomethacin stressor (assessing the effect of the stressor irrespective of the test period) Linear Mixed Models were used to take into account multiple measurements (i.e. four) per subject. To compare the effect of the 7-day supplementation of the three Lactobacilli versus placebo on urinary L/R ratio, a delta score was calculated, i.e. the L/R ratio after 7-day supplementation and with stress test minus L/R ratio of baseline with stress test. Related samples Wilcoxon Signed Rank test was used to assess differences between the interventions versus placebo and to test differences in $L / R$ ratio between the time points per test period. A two-sided $p$-value of $<0.05$ was considered statistically significant.

\section{Results}

Ten healthy volunteers, 7 female and 3 male, with the age between 19 and 48 years (mean 26.3 \pm 10.1 ) and a BMI between 16 and $24 \mathrm{~kg} / \mathrm{m}^{2}$ (mean 21.8 \pm 2.40 ) were included 
in the trial. None of the participants reported discomfort or possible side effects during the test and follow-up period, nor have we found any significant differences in the outcomes of the self-report questionnaires, i.e. GSRS and daily symptom diary, before and after supplementation or between the placebo and the three $L$. plantarum test periods (data not shown). One subject did not undergo one of the four gastroduodenoscopies (during the L. plantarum TIFN101 test period) due to anticipation of discomfort. These data were considered as missing during analyses.

\section{Intestinal permeability}

$L / R$ ratio of the baseline test with indomethacin stressor (mean $0.098 \pm 0.056$ ) was significantly higher compared to baseline L/R ratio without stressor $(0.058 \pm 0.037$, $\mathrm{p}<0.001$ ) (Figure 3.2), particularly due to a strong increase in urinary lactulose excretion. Comparing L/R ratio between these two time points per intervention period, the $L / R$ ratio increased significantly during three of the four periods (Table 3.1 and Supplementary Figure S3.I). For the L. plantarum WCFS1 test period this difference was not statistically significant. Furthermore, there was no statistically significant difference between $L / R$ ratios at baseline after the indomethacin induced stress compared to this ratio assessed after 7-day supplementation with either placebo or one of the three $L$. plantarum strains, measured after the indomethacin induced stress. This was also true for delta L/R ratio differences between test periods (Figure 3.3).

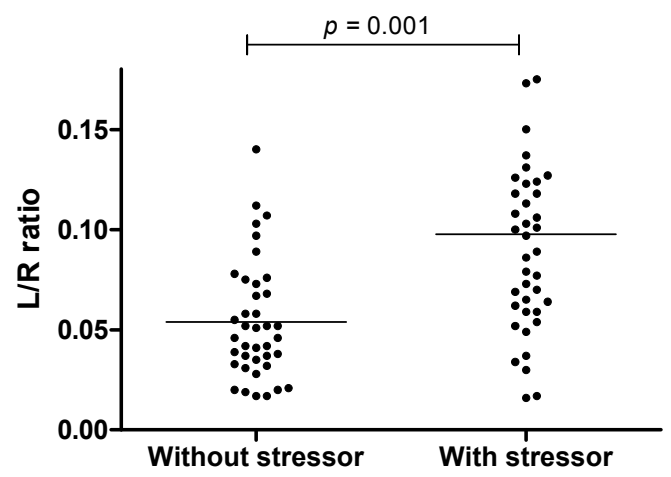

Figure 3.2 Indomethacin significantly increases intestinal permeability. Study participants were subjected to a sugar-based permeability test. Ratio of lactulose and rhamnose (L/R ratio) was determined in $5 \mathrm{hr}$ urine samples of subjects without indomethacin stressor (day -3) or with stressor (day 0 ) before intake of the permeability test. Mean \pm SD of $L / R$ ratio without stressor $=0.058 \pm 0.037$ and with stressor $=0.098 \pm 0.056$. Differences tested with Linear Mixed Models, adjusting for repeated measurements for each subject. 
Table $3.1 \quad$ Permeability test per intervention.

\begin{tabular}{lccc}
\hline Intervention & $\begin{array}{c}\text { Baseline, without } \\
\text { indomethacin stressor }\end{array}$ & $\begin{array}{c}\text { Baseline, with } \\
\text { indomethacin stressor }\end{array}$ & $\begin{array}{c}\text { After 7-day supplementation, } \\
\text { with indomethacin stressor }\end{array}$ \\
\hline Placebo & $0.026[0.016 ; 0.037]$ & $0.060[0.030 ; 0.0 .76]^{\mathrm{a}}$ & $0.079[0.058 ; 0.123]^{\mathrm{a}}$ \\
L. plantarum WCFS1 $_{\text { }}$ & $0.023[0.015 ; 0.055]$ & $0.047[0.026 ; 0.066]$ & $0.076[0.035 ; 0.164]^{\mathrm{b}}$ \\
L. plantarum CIP104448 $^{\mathrm{b}}$. plantarum TIFN101 & $0.032[0.017 ; 0.051]$ & $0.069[0.026 ; 0.105]^{\mathrm{a}}$ & $0.075[0.053 ; 0.114]^{\mathrm{b}}$ \\
L. $^{\mathrm{b}}$ & $0.022[0.018 ; 0.039]$ & $0.057[0.030 ; 0.078]^{\mathrm{a}}$ & $0.065[0.045 ; 0.133]^{\mathrm{b}}$ \\
\hline
\end{tabular}

$\mathrm{L} / \mathrm{R}$ ratio presented as median [IQR]. Differences tested with related samples Wilcoxon signed rank test. ${ }^{a} p<0.05,{ }^{b} p<0.01$ vs. 'Baseline, without indomethacin stressor'. No statistically significant differences were observed between 'Baseline, with indomethacin stressor' vs. 'After 7-day supplementation, with indomethacin stressor'.

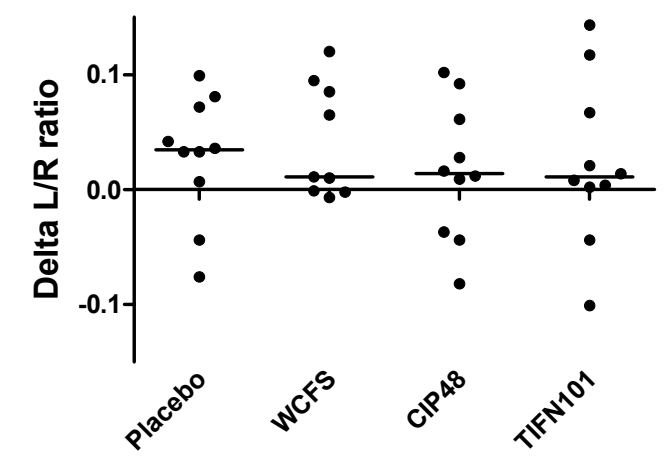

Figure 3.3 The effect of three L. plantarum strains and placebo on L/R ratio. Delta L/R ratio: L/R ratio after supplementation and stressor minus $L / R$ ratio before supplementation and stressor. Scatterplot with median line presented. No statistically significant difference found between test periods, tested with related samples Wilcoxon signed rank test.

\section{Immunofluorescence labelling: ZO-1 and occludin expression}

Immunofluorescent staining of the duodenal mucosa tissue samples showed expression of ZO-1 and occludin proteins at the apical part of epithelial cells, colocalized along the villous epithelium. Although the expression of both proteins seemed to increase after the interventions when compared to placebo (Figure 3.4), the differences did not reach statistically significance.

\section{Transcriptional responses of tight junction associated genes}

Microarray analysis of single gene transcriptome with focus on the tight junctions and supporting proteins (Supplementary Table S3.2) showed a significant down-regulation of the claudin 5 gene by L. plantarum WCFS1 (mean fold change (FC): $-1.168, p=0.019$ ) and the claudin 19 gene by CIP48 $(-1.146, p=0.036)$, and a significant up-regulation of the actinin alpha 4 gene by TIFN101 (1.105, $p=0.032)$. RT-qPCR was conducted for five 
genes, which confirmed the microarray analysis, showing no statistically significant differences for claudin 3, claudin 4, MLCK, ZO-1 and occludin gene transcription between bacterial strains and placebo (data not shown).
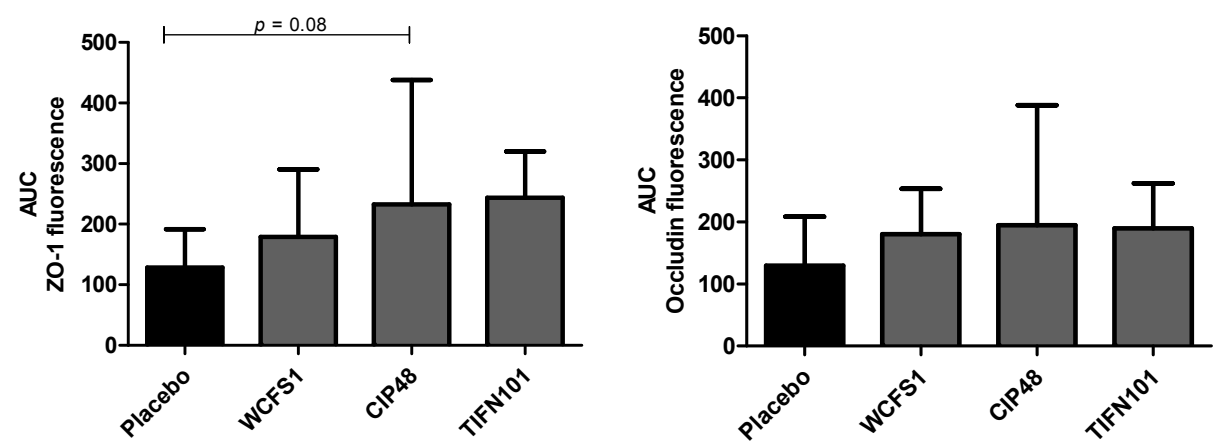

Figure 3.4 Zonula occludens-1 (ZO-1) and occludin fluorescence intensity in duodenum mucosal tissue after immunofluorescent staining, expressed as area under the curve (AUC), after treatment with placebo or one of the three L. plantarum strains.

\section{Transcriptional responses: Ingenuity Pathway Analysis}

To further explore possible effects of the L. plantarum strains on the mucosal barrier, we focused on canonical pathways related to mucosal structure, enterocyte energy supply, DNA-repair, stress response and cell activity. All three interventions induced changes in transcriptional responses in the indomethacin stressed duodenal mucosa when compared to placebo. Some of the described pathways below, are only available as online supplementary material, due to the size of the figures.

TIFN101 demonstrated regulation of pathways involved in remodelling of the mucosal structure. Epithelial-mesenchymal transition pathway (online supplementary material), involved in disruption and remodelling of desmosomal adhesion, and pathways that might be involved in restructuring of cell-cell adhesions (i.e. macropinocytosis signalling pathway, Supplementary Figure S3.2) and cytoskeleton rearrangement (i.e. integrin signalling pathway, Supplementary Figure S3.3) were upregulated. Moreover, we observed a combined up-regulation of matrix metalloproteinase 2 (MMP2) gene (1.297, $\mathrm{p}=0.006)$, involved in degradation of extracellular structures, and its inhibitors, so-called tissue inhibitors of metalloproteinase (TIMP) 1 (1.362, p=0.006), and TIMP3 (1.243, $p=0.028)$. TIFN101 also upregulated the muc2 gene $(1.286, p=0.031)$, encoding for the mucin 2 protein. Furthermore, the glutamate-ammonia ligase (GLUL) gene (1.155, $\mathrm{p}=0.022$ ), involved in glutamine biosynthesis pathways, was upregulated, which may enhance energy release based on glutamine biosynthesis of mucosal cells. On the other hand this strain downregulated expression of genes involved in the tricarboxylic acid 
(TCA) cycle II pathway (Supplementary Figure S3.4), which may lead to reduced adenosine triphosphate (ATP) production.

WCFS1 and CIP48 downregulated nitric oxide synthase 3 gene (NOS3) $(-1.113, p=0.039$ and $-1.162, p=0.018$, respectively), involved in cell-cell adhesion, and MMP9 $(-1.178$, $p=0.045$ and $-1.218, p=0.025$, respectively), involved in breakdown of the extracellular matrix. CIP48 upregulated genes related to inositol phosphates biosynthesis, components of the cytosolic side of the cell membrane, such as myotubularin related protein 9 (MTMR9) (1.132, $p=0.014), \operatorname{MTMR8}$ (1.182, $p=0.043)$, MTMR2 (1.131, $\mathrm{p}=0.006)$, phospholipase-C $\beta 4$ (PLCB4) (1.131, $\mathrm{p}=0.014)$, and protein tyrosine phosphatase, non-receptor type 2 (PTPN2) $(1.187, \mathrm{p}=0.018)$.

WCFS1 demonstrated regulation of genes and pathways related to cell division processes and DNA repair (online supplementary material), with involvement of BRCA1 (1.225, $p=0.022)$ and BRCA2 genes $(1.199, p=0.018)$, and upregulation of protection of telomere1 (POT1) gene $(1.161, \mathrm{p}=0.004)$. CIP48 showed similar effects, with upregulation of BRCA1 (1.182, $p=0.043)$ and $2(1.199, p=0.018)$ genes and a pathway involved in cell division and DNA-repair (Supp. Figure S3.3-e). Finally, CIP48 also inhibited expression of the GADD45B gene $(-1.223, p=0.034)$ in the glutathionemediated detoxification pathway, involved in cell stress response, and the neuropeptide $Y$ (NPY) gene $(-1.159, p=0.022)$, a protein that influences the fat metabolism.

\section{Discussion}

In the current study we investigated the effects of oral intake of three L. plantarum strains over a 7-day period on small intestinal barrier function under a stressed condition, induced by intake of NSAIDs, in healthy volunteers. Small intestinal permeability was increased after intake of indomethacin, but was not significantly affected by any of the treatments. However, gene transcription pathway analysis clearly demonstrated that the different interventions modulated mucosal structure, enterocyte energy supply, DNA-repair, stress response and cell activity pathways, when compared to placebo, revealing new insights into the mechanisms by which live potentially probiotic organisms may modulate human small intestinal mucosa.

Intake of indomethacin at baseline and after the intervention period resulted in a significant increase in small intestinal permeability, as reflected by the increased urinary excretion of lactulose versus rhamnose. This confirmed that the NSAID is a potent stressor of small intestinal barrier function, and that this model is well suited to investigate potential beneficial effects of bioactive substances in healthy individuals. The 7-day supplementation period of the different bacterial strains did not significantly affect small intestinal permeability. Previously, Karczewski et al. demonstrated that a 
continuous intraduodenal administration of L. plantarum WCFS1 over a 6-hr period in healthy subjects (without intake of NSAIDs) increased tight junction-linked localisation of occludin and ZO-1 proteins. ${ }^{16}$ Our results do not confirm these findings, but our design is different because we used indomethacin as stressor. The influence of indomethacin on epithelial barrier may have been too pronounced to detect smaller potentially beneficial effects induced by the bacterial interventions. However, also differences in route of administration (i.e. oral versus intraduodenal) and exposure time (i.e. 7 days versus 6 hours) may account for the observed difference between these studies.

Although the interventions did not affect indomethacin induced changes in small intestinal permeability, the three tested $L$. plantarum strains may influenced intrinsic repair processes of the small intestinal mucosa. Differential effects on genes and pathways involved in mucosal structure maintenance have been observed. The strongest modulatory effects in this respect were demonstrated by $L$. plantarum TIFN101 when compared to placebo. Intake of this bacterial strain influenced the transcriptional response related to the turnover of adhesion molecules and homeostasis of the mucus layer. This is exemplified by alterations of pathways involved in cytoskeleton rearrangement, and upregulation of genes such as actinin alpha 4, several MMP genes but also their inhibitors (TIMP genes). Moreover, the up-regulation of the macropinocytosis signalling pathway, which is one of the mechanisms involved in disruption of intercellular contacts of adherens and tight junctions, ${ }^{37,38}$ may lead to increased recycling of adhesive molecules. Finally, the mucin 2 gene is upregulated by TIFN101. Mucin 2 is produced by the goblet cells and together with related mucin proteins is polymerized into insoluble mucus, which is an important part of the intestinal barrier. As the number of goblet cells, and thereby mucin production, is increasing along the proximal to distal axis of the intestine, the effect of TIFN101 on intestinal barrier might also be stronger when it reaches more distal parts of the intestine.

L. plantarum WCFS1 showed some negative effects on the transcription of genes and pathways involved in mucosal barrier homeostasis, by downregulation of genes such as claudin $5^{39}$ and NOS3. The latter gene is linked to maintenance of cytoskeletal proteins, e.g. $\alpha$-tubulin, actinin and vimentin, and tight junction integral membrane proteins, e.g. occludin. ${ }^{40}$ Contradictory, L. plantarum WCFS1 downregulated MMP9 gene, which leads to inhibitory effects on breakdown of extracellular matrix, ${ }^{41}$ suggesting beneficial effects of $L$. plantarum WCFS1 supplementation on mucosal integrity. L. plantarum CIP48 demonstrated similar modest effects on gene transcription. NOS3, MMP9, and claudin 19 genes were downregulated after the intervention. Furthermore, genes involved in inositol phosphates biosynthesis, important for cell membrane structure and membrane potential, ${ }^{42}$ was upregulated by L. plantarum CIP48. Thereby, both strains, L. plantarum WCFS1 and CIP48, seem to be involved in maintaining a balance 
between enhancement and disruption of cell-cell adhesion and mucosal structure, with no direct functional consequences for intestinal permeability and tight junction protein expression.

We identified more mechanisms by which the three tested L. plantarum strains affect the human host on transcriptome level. L. plantarum WCFS1 increased the transcription of genes such as BRCA1 and 2 and POT1, which are involved in cell division processes, DNA repair and telomere length regulation, suggesting that this bacterial strain may be involved in the enhancement of epithelial healing after indomethacin induced mucosal stress. L. plantarum TIFN101 modulated genes that may be involved in inducing a shift of energy supply of the mucosal cells from oxidative phosphorylation (by TCA II pathway downregulation) towards glutamine production (by increased transcription of glutamine biosynthesis pathways). Glutamine is an important energy source for intestinal epithelial cells and it promotes cell differentiation and proliferation. ${ }^{43}$ Moreover, glutamine supplementation has been reported to reinforce the intestinal epithelial mucosal barrier. ${ }^{44}$ Finally, the transcription of the NPY gene, which encodes for a neuro-hormone in the leptin signaling pathway was inhibited by $L$. plantarum CIP48. Antagonising NPY-ergic activity has been reported to reduce food intake. ${ }^{45}$ However, no other effects on satiety related mechanisms were observed in the transcriptome analysis, and therefore the effects on satiety are probably minimal.

In summary, L. plantarum TIFN101 demonstrated strong regulation of genes and pathways involved in cell-cell adhesion and turnover of adhesion molecules. Furthermore, this strain seems to be able to induce alterations in energy supply of the mucosal cells by affecting both oxidative phosphorylation- and glutamine production pathways. L. plantarum WCFS1 modulated the transcription of genes related to DNA repair, possibly based on mucosal tissue healing upon NSAID induced stress, and showed minor negative effects on gene transcription related to mucosal structure. $L$. plantarum CIP48 demonstrated similar effects with respect to DNA repair mechanisms and minor positive effects on mucosal structure.

Overall our study demonstrates that the effect of bacterial supplementation on gene transcription is highly strain dependent and differs considerably within the same species. In the current study the transcriptome analysis was performed on duodenal biopsies. The effects might even be more pronounced or at least different in more distal parts of the intestine, as the mucosal function along the longitudinal axis of the intestine does vary. Further research is needed to investigate this hypothesis.

In previous studies from our consortium L. plantarum WCFS1 was found to modulate multiple pathways in duodenal mucosa, related to lipid biosynthesis, cellular proliferation, cell-death and immune responses. ${ }^{25,30}$ In the present study, we were able to confirm that $L$. plantarum WCFS1 does affect some similar pathways related to cell 
function; however, most transcriptional responses were dissimilar. The main difference between the current and previous research was the intake of indomethacin in the present study. While in previous research the effects of $L$. plantarum WCFS1 on healthy intestinal function were investigated, we assessed effect of the three bacterial strains on small intestinal mucosa under a stressed condition. Furthermore, Troost et al. administrated a load of L. plantarum WCFS1 using oro- and nasogastric catheters during 1 and 6 hours, $^{30}$ and Van Baarlen et al. administered supplements of the same bacterium by oral intake, and assessed effects after 6 hours. ${ }^{25}$ Therefore, besides the difference in administration route (compared to Troost et al.), ${ }^{30}$ we measured long term effects (i.e. after a 7-day administration period) of the bacterial strains intake, compared to the short term effects (i.e. after 1 and 6 hours) assessed in the previous studies. Troost et al. demonstrated that different supplementation periods lead to different transcriptional responses even between one and six hour administration of the bacteria. ${ }^{30}$ The current data, in combination with previous findings, demonstrate that not only the selection of the bacterial species or even strains, but also the presence or absence of intestinal mucosal distress of the consumer, as well as route and time period of administration of viable microbes may induce different effects on human health. The current study contributes to further understanding of complex hostmicrobe interactions and human intestinal homeostasis, providing leads for future research in this field.

\section{Conclusion}

Application of the NSAID indomethacin induced a profound increase in small intestinal permeability, which was not significantly affected by the intake of the three $L$. plantarum strains. However, the tested bacterial strains did modulate intrinsic repair processes on mucosal gene transcription level. L. plantarum TIFN101 demonstrated the most pronounced beneficial effects, by among others modulation of genetranscriptions that are related to mucosal structure. L. plantarum WCFS1 and CIP48 induced more moderate transcriptional effects with respect to mucosal integrity. This illustrates the strain dependency with respect to inducing specific effects on gene transcription in human intestinal mucosa. 


\section{References}

1. Turner JR. Intestinal mucosal barrier function in health and disease. Nat Rev Immunol. 2009;9:799-809

2. Ahrne S, Hagslatt ML. Effect of lactobacilli on paracellular permeability in the gut. Nutrients. 2011;3:104-17.

3. Camilleri M, Madsen K, Spiller R, Greenwood-Van Meerveld B, Verne GN. Intestinal barrier function in health and gastrointestinal disease. Neurogastroenterol Motil. 2012;24:503-12.

4. Salim SY, Soderholm JD. Importance of disrupted intestinal barrier in inflammatory bowel diseases. Inflamm Bowel Dis. 2011;17:362-81.

5. Vivinus-Nébot M, Frin-Mathy G, Bzioueche H, Dainese R, Bernard G, Anty R, Filippi J, Saint-Paul MC, Tulic MK, Verhasselt V, Hébuterne X, Piche T. Functional bowel symptoms in quiescent inflammatory bowel diseases: role of epithelial barrier disruption and low-grade inflammation. Gut. 2014;63:744-52.

6. Heyman M, Abed J, Lebreton C, Cerf-Bensussan N. Intestinal permeability in coeliac disease: insight into mechanisms and relevance to pathogenesis. Gut. 2012;61:1355-64.

7. Martínez C, Lobo B, Pigrau M, Ramos L, González-Castro AM, Alonso C, Guilarte M, Guilá M, de Torres I, Azpiroz F, Santos J, Vicario M. Diarrhoea-predominant irritable bowel syndrome: an organic disorder with structural abnormalities in the jejunal epithelial barrier. Gut. 2013;62:1160-8.

8. Mujagic Z, Ludidi S, Keszthelyi D, Hesselink MA, Kruimel JW, Lenaerts K, et al. Small intestinal permeability is increased in diarrhoea predominant IBS, while alterations in gastroduodenal permeability in all IBS subtypes are largely attributable to confounders. Aliment Pharmacol Ther. 2014; 40:288-97.

9. Bosi E, Molteni L, Radaelli MG, Folini L, Fermo I, Bazzigaluppi E, Piemonti L, Pastore MR, Paroni R. Increased intestinal permeability precedes clinical onset of type 1 diabetes. Diabetologia. 2006;49:2824-7.

10. Barba A, Schena D, Andreaus MC, Faccini G, Pasini F, Brocco G, Cavallini G, Scuro LA, Chieregato GC. Intestinal permeability in patients with atopic eczema. Br J Dermatol. 1989;120:71-5.

11. Cariello R, Federico A, Sapone A, Tuccillo C, Scialdone VR, Tiso A, Miranda A, Portincasa P, Carbonara V, Palasciano G, Martorelli L, Esposito P, Cartenì M, Del Vecchio Blanco C, Loguercio C. Intestinal permeability in patients with chronic liver diseases: Its relationship with the aetiology and the entity of liver damage. Dig Liver Dis. 2010;42:200-4.

12. Kerckhoffs AP, Akkermans LM, de Smet MB, Besselink MG, Hietbrink F, Bartelink IH, Busschers WB, Samsom M, Renooij W. Intestinal permeability in irritable bowel syndrome patients: effects of NSAIDs. Dig Dis Sci. 2010;55:716-23.

13. Troost FJ, Saris WH, Brummer RJ. Recombinant human lactoferrin ingestion attenuates indomethacininduced enteropathy in vivo in healthy volunteers. Eur J Clin Nutr. 2003;57:1579-85.

14. Bjarnason I, Williams P, Smethurst P, Peters TJ, Levi AJ. Effect of non-steroidal anti-inflammatory drugs and prostaglandins on the permeability of the human small intestine. Gut. 1986;27:1292-7.

15. Odenwald MA, Turner JR. Intestinal permeability defects: is it time to treat? Clin Gastroenterol Hepatol. 2013;11:1075-83.

16. Karczewski J, Troost FJ, Konings I, Dekker J, Kleerebezem M, Brummer RJ, Wells JM. Regulation of human epithelial tight junction proteins by Lactobacillus plantarum in vivo and protective effects on the epithelial barrier. Am J Physiol Gastrointest Liver Physiol. 2010;298:G851-9.

17. Mao Y, Nobaek S, Kasravi B, Adawi D, Stenram U, Molin G, Jeppsson B. The effects of Lactobacillus strains and oat fiber on methotrexate-induced enterocolitis in rats. Gastroenterology. 1996;111:334-44.

18. White JS, Hoper M, Parks RW, Clements WD, Diamond T, Bengmark S. The probiotic bacterium Lactobacillus plantarum species 299 reduces intestinal permeability in experimental biliary obstruction. Lett Appl Microbiol. 2006;42:19-23.

19. Mennigen R, Nolte K, Rijcken E, Utech M, Loeffler B, Senninger N, et al. Probiotic mixture VSL\#3 protects the epithelial barrier by maintaining tight junction protein expression and preventing apoptosis in a murine model of colitis. Am J Physiol Gastrointest Liver Physiol. 2009;296:G1140-9.

20. Mangell P, Nejdfors P, Wang M, Ahrne S, Westrom B, Thorlacius $H$, et al. Lactobacillus plantarum 299v inhibits Escherichia coli-induced intestinal permeability. Dig Dis Sci. 2002;47:511-6. 
21. Zeng J, Li YQ, Zuo XL, Zhen YB, Yang J, Liu CH. Clinical trial: effect of active lactic acid bacteria on mucosal barrier function in patients with diarrhoea-predominant irritable bowel syndrome. Aliment Pharmacol Ther. 2008;28:994-1002.

22. Shanahan F, Quigley EM. Manipulation of the microbiota for treatment of IBS and IBD-challenges and controversies. Gastroenterology. 2014;146:1554-63.

23. Meijerink $M$, van Hemert S, Taverne $N$, Wels $M$, de Vos $P$, Bron PA, Savelkoul HF, van Bilsen J, Kleerebezem M, Wells JM. Identification of genetic loci in Lactobacillus plantarum that modulate the immune response of dendritic cells using comparative genome hybridization. PloS One. 2010;5:e10632.

24. van Hemert S, Meijerink M, Molenaar D, Bron PA, de Vos P, Kleerebezem M, Wells JM, Marco ML. Identification of Lactobacillus plantarum genes modulating the cytokine response of human peripheral blood mononuclear cells. BMC Microbiol. 2010;10:293.

25. van Baarlen P, Troost FJ, van Hemert S, van der Meer C, de Vos WM, de Groot PJ, Hooiveld GJ, Brummer RJ, Kleerebezem M. Differential NF-kappaB pathways induction by Lactobacillus plantarum in the duodenum of healthy humans correlating with immune tolerance. Proc Natl Acad Sci U S A. 2009;106:2371-6.

26. Vogt LM, Meyer D, Pullens G, Faas MM, Venema K, Ramasamy U, et al. Toll-like receptor 2 activation by beta2-->1-fructans protects barrier function of T84 human intestinal epithelial cells in a chain lengthdependent manner. J Nutr. 2014;144:1002-8.

27. Kleerebezem M, Boekhorst J, van Kranenburg R, Molenaar D, Kuipers OP, Leer R, Tarchini R, Peters SA, Sandbrink HM, Fiers MW, Stiekema W, Lankhorst RM, Bron PA, Hoffer SM, Groot MN, Kerkhoven R, de Vries M, Ursing B, de Vos WM, Siezen RJ. Complete genome sequence of Lactobacillus plantarum WCFS1. Proc Natl Acad Sci U S A. 2003 Feb 18;100:1990-5.

28. Vesa T, Pochart P, Marteau P. Pharmacokinetics of Lactobacillus plantarum NCIMB 8826, Lactobacillus fermentum KLD, and Lactococcus lactis MG 1363 in the human gastrointestinal tract. Aliment Pharmacol Ther. 2000 Jun;14(6):823-8.

29. Smelt MJ, de Haan BJ, Bron PA, van Swam I, Meijerink M, Wells JM, Faas MM, de Vos P. L. plantarum, L. salivarius, and $L$. lactis attenuate Th2 responses and increase Treg frequencies in healthy mice in a strain dependent manner. PLoS One. 2012;7:e47244.

30. Troost FJ, van Baarlen P, Lindsey $P$, Kodde A, de Vos WM, Kleerebezem M, Brummer RJ. Identification of the transcriptional response of human intestinal mucosa to Lactobacillus plantarum WCFS1 in vivo. BMC Genomics. 2008;9:374.

31. Svedlund J, Sjodin I, Dotevall G. GSRS--a clinical rating scale for gastrointestinal symptoms in patients with irritable bowel syndrome and peptic ulcer disease. Dig Dis Sci. 1988;33:129-34.

32. van Wijck K, van Eijk HM, Buurman WA, Dejong $\mathrm{CH}$, Lenaerts K. Novel analytical approach to a multisugar whole gut permeability assay. J Chromatogr B Analyt Technol Biomed Life Sci. 2011;879: 2794-801.

33. Lin K, Kools H, de Groot PJ, Gavai AK, Basnet RK, Cheng F, Wu J, Wang X, Lommen A, Hooiveld GJ, Bonnema G, Visser RG, Muller MR, Leunissen JA. MADMAX - Management and analysis database for multiple omics experiments. J Integr Bioinform. 2011;8:160.

34. Keszthelyi D, Troost FJ, Jonkers DM, van Eijk HM, Lindsey PJ, Dekker J, et al. Serotonergic reinforcement of intestinal barrier function is impaired in irritable bowel syndrome. Aliment Pharmacol Ther. 2014;40:392-402.

35. Elamin E, Masclee A, Dekker J, Jonkers D. Ethanol disrupts intestinal epithelial tight junction integrity through intracellular calcium-mediated Rho/ROCK activation. Am J Physiol Gastrointest Liver Physiol. 2014;306:G677-85.

36. Abramoff MD. Image processing with ImageJ. Biophotonics Int. 2004;11:36-42.

37. Ivanov Al, Nusrat A, Parkos CA. Endocytosis of epithelial apical junctional proteins by a clathrinmediated pathway into a unique storage compartment. Mol Biol Cell. 2004;15:176-88.

38. Feick P, Haas SR, Singer MV, Bocker U. Low-dose exposure of intestinal epithelial cells to formaldehyde results in MAP kinase activation and molecular alteration of the focal adhesion protein paxillin. Toxicology. 2006;219:60-72.

39. Kim $\mathrm{CY}$, Kim KH. Curcumin prevents leptin-induced tight junction dysfunction in intestinal Caco-2 BBe cells. J Nutr Biochem. 2014;25:26-35. 
40. Lee NP, Cheng CY. Regulation of Sertoli cell tight junction dynamics in the rat testis via the nitric oxide synthase/soluble guanylate cyclase/3',5'-cyclic guanosine monophosphate/protein kinase $\mathrm{G}$ signaling pathway: an in vitro study. Endocrinology. 2003;144:3114-29.

41. Bhattacharyya S, Tobacman JK. Arylsulfatase B regulates colonic epithelial cell migration by effects on MMP9 expression and RhoA activation. Clin Exp Metastasis. 2009;26:535-45.

42. Dakin K, Li WH. Cell membrane permeable esters of D-myo-inositol 1,4,5-trisphosphate. Cell Calcium. 2007;42:291-301.

43. Rhoads M. Glutamine signaling in intestinal cells. JPEN J Parenter Enteral Nutr. 1999;23(5 Suppl): S38-40.

44. Vermeulen MA, de Jong J, Vaessen MJ, van Leeuwen PA, Houdijk AP. Glutamate reduces experimental intestinal hyperpermeability and facilitates glutamine support of gut integrity. World J Gastroenterol. 2011;17:1569-73.

45. Hanson ES, Dallman MF. Neuropeptide Y (NPY) may integrate responses of hypothalamic feeding systems and the hypothalamo-pituitary-adrenal axis. J Neuroendocrinol. 1995;7:273-9. 


\section{Supplemental data}

Table S3.1 Randomization schedule.

\begin{tabular}{lcccc}
\hline Subject number & Test period 1 & Test period 2 & Test period 3 & Test period 4 \\
\hline 001 & c & a & b & d \\
002 & a & d & c & b \\
003 & b & c & d & a \\
004 & d & a & c & b \\
005 & c & d & a & b \\
006 & a & b & d & c \\
007 & b & d & c & a \\
008 & d & b & a & c \\
009 & c & b & a & d \\
010 & a & c & b & d \\
\hline
\end{tabular}

Subject numbers (and test period schedules) were assigned to participants in chronological order of inclusion. The supplements were distributed under double-blind conditions. The codes represented the following supplements:, $\mathrm{a}=$ placebo, $\mathrm{b}=$ L. plantarum WCFS1, $\mathrm{c}=$ L. plantarum CIP4448, $\mathrm{d}=$ L. plantarum TIFN101.

Table S3.2 List of tight junction, adherens junction and associated genes.

\begin{tabular}{|c|c|c|c|c|c|c|c|}
\hline Gene ID & Official Full Name & $\begin{array}{l}\text { WCFS1 } \\
\text { Mean FC }\end{array}$ & $\mathrm{p}$-value & $\begin{array}{c}\text { CIP48 } \\
\text { Mean FC }\end{array}$ & p-value & $\begin{array}{c}\text { TIFN101 } \\
\text { Mean } \\
\text { FC }\end{array}$ & p-value \\
\hline 7082 & $\begin{array}{l}\text { tight junction protein } 1 \\
\text { (zonula occludens 1) }\end{array}$ & 1.09 & 0.13 & 1.06 & 0.21 & 1.04 & 0.52 \\
\hline 9414 & $\begin{array}{l}\text { tight junction protein } 2 \\
\text { (zonula occludens } 2 \text { ) }\end{array}$ & 1.07 & 0.17 & 1.06 & 0.23 & -1.02 & 0.55 \\
\hline 27134 & $\begin{array}{l}\text { tight junction protein } 3 \\
\text { (zonula occludens } 3 \text { ) }\end{array}$ & -1.03 & 0.69 & -1.05 & 0.50 & -1.02 & 0.79 \\
\hline 9076 & claudin 1 & -1.07 & 0.34 & -1.01 & 0.80 & -1.06 & 0.50 \\
\hline 9075 & claudin 2 & -1.18 & 0.56 & -1.04 & 0.67 & -1.17 & 0.24 \\
\hline 1365 & claudin 3 & 1.02 & 0.89 & -1.09 & 0.47 & 1.11 & 0.40 \\
\hline 1364 & claudin 4 & 1.08 & 0.50 & 1.04 & 0.87 & 1.08 & 0.36 \\
\hline 7122 & claudin 5 & $-1.17^{*}$ & 0.02 & -1.03 & 0.60 & -1.04 & 0.48 \\
\hline 9074 & claudin 6 & 1.02 & 0.77 & -1.02 & 0.89 & -1.00 & 0.85 \\
\hline 1366 & claudin 7 & 1.03 & 0.62 & -1.02 & 0.81 & 1.07 & 0.24 \\
\hline 9073 & claudin 8 & -1.07 & 0.18 & -1.08 & 0.13 & -1.05 & 0.23 \\
\hline 9080 & claudin 9 & 1.04 & 0.51 & -1.09 & 0.17 & -1.03 & 0.73 \\
\hline 9071 & claudin 10 & -1.22 & 0.13 & 1.01 & 0.93 & -1.10 & 0.57 \\
\hline 5010 & claudin 11 & -1.03 & 0.73 & -1.05 & 0.46 & -1.02 & 0.79 \\
\hline 9069 & claudin 12 & 1.00 & 0.98 & 1.03 & 0.61 & -1.00 & 0.93 \\
\hline 23562 & claudin 14 & -1.08 & 0.21 & -1.03 & 0.71 & -1.04 & 0.52 \\
\hline 24146 & claudin 15 & -1.01 & 0.93 & -1.06 & 0.48 & 1.09 & 0.31 \\
\hline 10686 & claudin 16 & 1.02 & 0.66 & -1.02 & 0.81 & 1.00 & 0.79 \\
\hline 26285 & claudin 17 & -1.07 & 0.26 & -1.11 & 0.12 & -1.10 & 0.07 \\
\hline 149461 & claudin 19 & -1.11 & 0.11 & $-1.15^{*}$ & 0.04 & -1.02 & 0.72 \\
\hline 49861 & claudin 20 & -1.00 & 0.96 & 1.03 & 0.49 & 1.06 & 0.31 \\
\hline 100132463 & claudin 24 & -1.02 & 0.56 & 1.03 & 0.36 & -1.01 & 0.74 \\
\hline 56650 & $\begin{array}{l}\text { claudin domain } \\
\text { containing } 1\end{array}$ & 1.03 & 0.54 & 1.00 & 0.97 & -1.05 & 0.30 \\
\hline
\end{tabular}


Table S3.2 (continued)

\begin{tabular}{|c|c|c|c|c|c|c|c|}
\hline Gene ID & Official Full Name & $\begin{array}{l}\text { WCFS1 } \\
\text { Mean FC }\end{array}$ & $\mathrm{p}$-value & $\begin{array}{c}\text { CIP48 } \\
\text { Mean FC }\end{array}$ & p-value & $\begin{array}{c}\text { TIFN101 } \\
\text { Mean } \\
\text { FC }\end{array}$ & $\mathrm{p}$-value \\
\hline 100506658 & occludin & 1.06 & 0.36 & -1.03 & 0.68 & -1.03 & 0.51 \\
\hline 4638 & myosin light chain kinase & 1.01 & 0.78 & 1.10 & 0.09 & 1.03 & 0.51 \\
\hline 85366 & $\begin{array}{l}\text { myosin light chain kinase } \\
\text { family. member } 2\end{array}$ & -1.08 & 0.11 & -1.07 & 0.16 & -1.07 & 0.16 \\
\hline 91807 & $\begin{array}{l}\text { myosin light chain kinase } \\
\text { family. member } 3\end{array}$ & -1.06 & 0.24 & -1.03 & 0.60 & 1.01 & 0.99 \\
\hline 340156 & $\begin{array}{l}\text { myosin light chain kinase } \\
\text { family. member } 4\end{array}$ & 1.02 & 0.69 & 1.05 & 0.33 & -1.00 & 0.94 \\
\hline 103910 & $\begin{array}{l}\text { myosin. light chain } 12 \mathrm{~B} \text {. } \\
\text { regulatory }\end{array}$ & -1.05 & 0.44 & -1.05 & 0.42 & -1.06 & 0.30 \\
\hline 87 & actinin. alpha 1 & 1.03 & 0.69 & 1.01 & 0.99 & 1.15 & 0.05 \\
\hline 88 & actinin. alpha 2 & 1.01 & 0.82 & -1.03 & 0.52 & -1.02 & 0.63 \\
\hline 89 & actinin. alpha 3 & -1.03 & 0.59 & -1.01 & 0.70 & 1.03 & 0.49 \\
\hline 81 & actinin. alpha 4 & 1.05 & 0.31 & -1.05 & 0.27 & $1.11 *$ & 0.03 \\
\hline 5934 & $\begin{array}{l}\text { retinoblastoma-like } 2 \\
(\mathrm{P} 130)\end{array}$ & 1.07 & 0.23 & 1.02 & 0.66 & 1.02 & 0.74 \\
\hline 6709 & $\begin{array}{l}\text { spectrin. alpha. non- } \\
\text { erythrocytic } 1\end{array}$ & -1.03 & 0.60 & 1.02 & 0.65 & 1.02 & 0.75 \\
\hline 57530 & cingulin & 1.04 & 0.57 & -1.03 & 0.85 & 1.01 & 0.97 \\
\hline 8189 & symplekin & -1.01 & 0.97 & -1.07 & 0.23 & 1.02 & 0.67 \\
\hline 999 & E-cadherin & -1.01 & 0.85 & -1.02 & 0.75 & 1.00 & 0.82 \\
\hline
\end{tabular}

Mean fold-change in expression of the three $L$. plantarum strains when compared to placebo and raw $p$-value calculated using the IBMT (Intensity-Based Moderated T-statistic) regularised paired t-test versus placebo. Statistically significant up- or downregulation presented in bold and with a *. 


\section{Placebo}

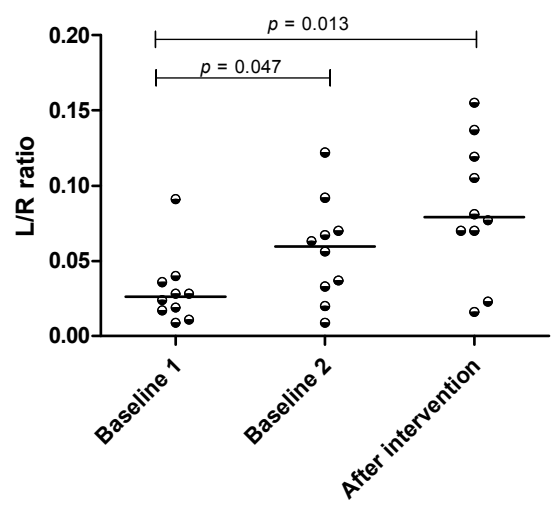

\section{L. plantarum CIP104448}

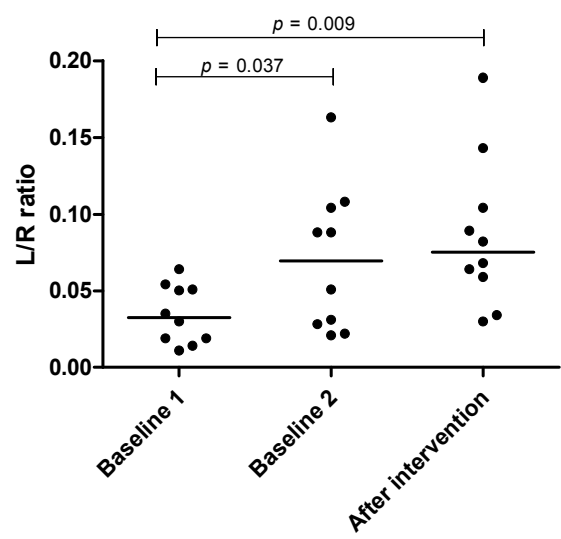

L. plantarum WCFS

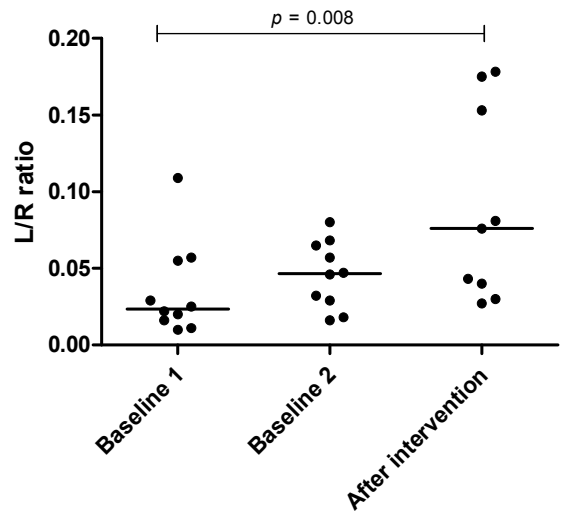

L. plantarum TIFN101

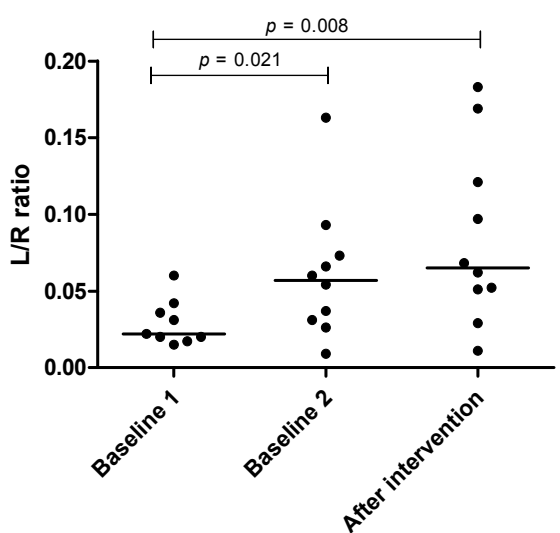

Figure S3.1 L/R ratio in 0-5h urine; small intestinal permeability. Intestinal permeability test before, i.e. day -3 (baseline 1), day 0 (baseline 2), and 7 days after start of the intervention. During the latter two time points subjects were challenged with indomethacin. Scatterplot with median L/R ratio presented. Differences tested with related samples Wilcoxon signed rank test. 


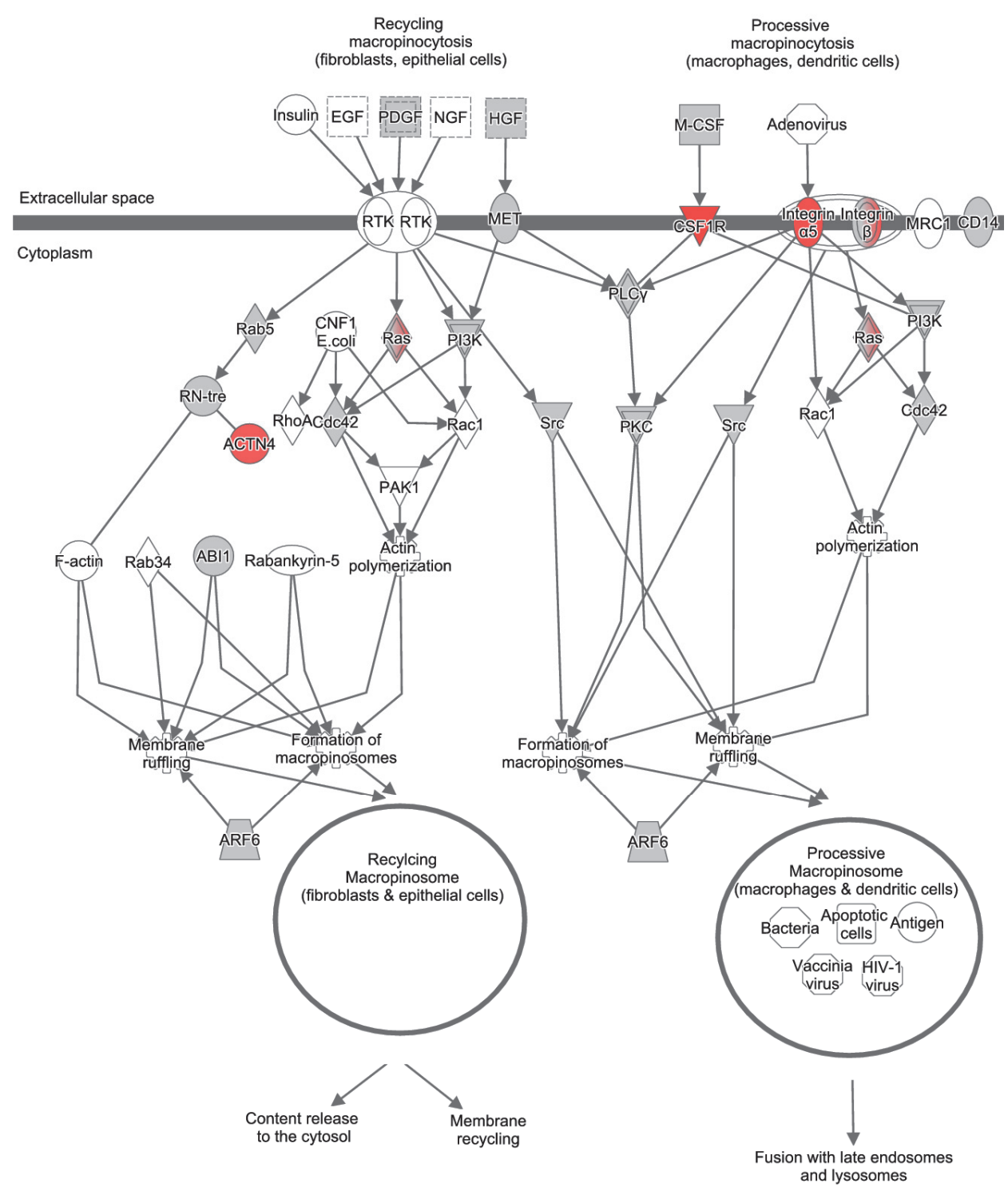

Figure S3.2 Macropinocytosis signalling pathway, upregulated by L. plantarum TIFN 101, compared to placebo. Green and red coloured genes indicate down or upregulation, respectively. Grey indicates the regulation did not reach significance. 

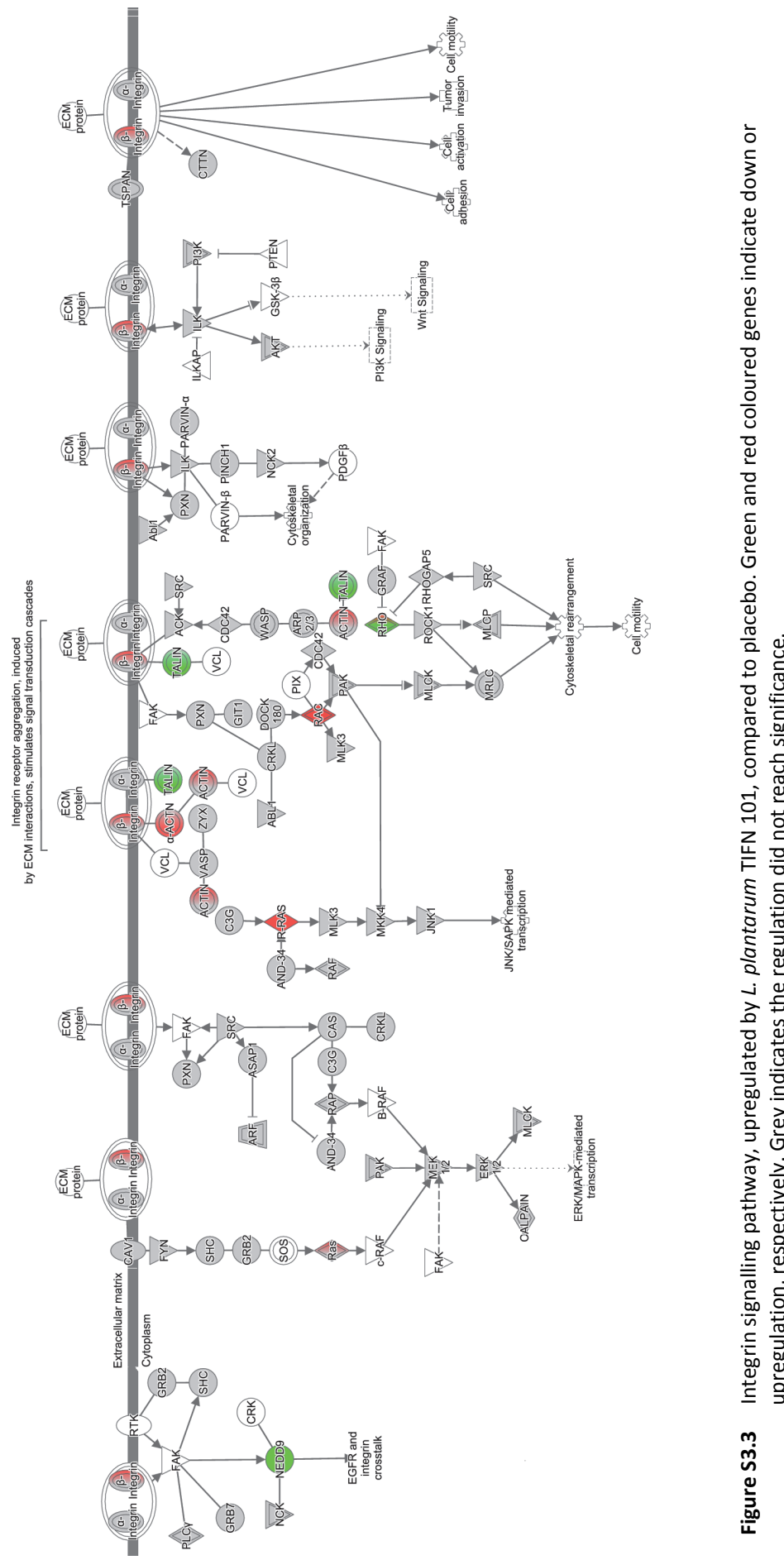


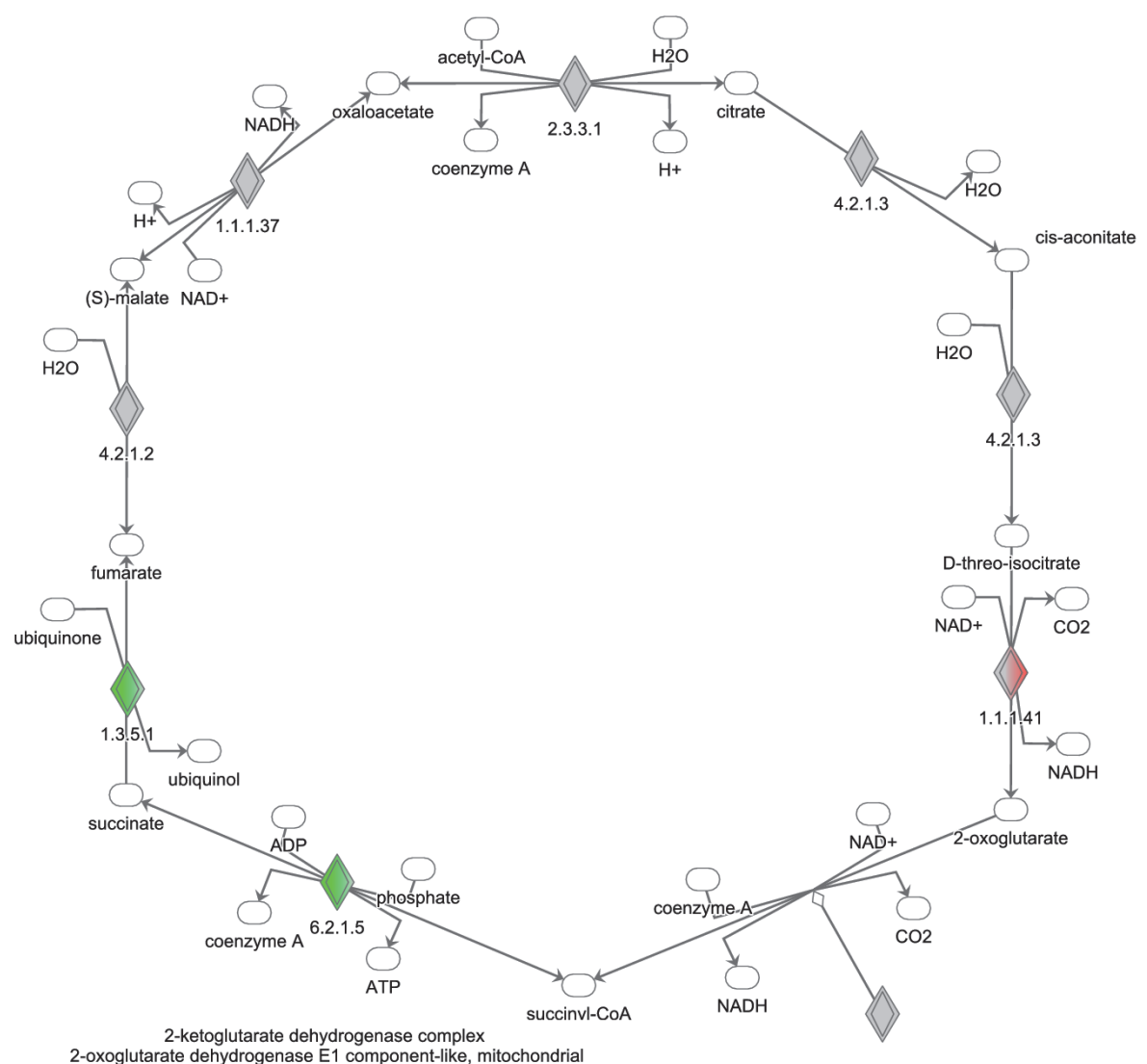

Probable 2-oxoglutarate dehydrogenase E1 component DHKTD1, mitochondrial

Figure S3.4 Tricarboxylic acid (TCA) cycle II pathway, upregulated by L. plantarum TIFN 101, compared to placebo. Green and red coloured genes indicate down or upregulation, respectively. Grey indicates the regulation did not reach significance. 


\section{Chapter 4}

\section{Effects of $L$. plantarum on immunity}

Differential human mucosal transcriptomic and peripheral immune responses

induced by three Lactobacillus plantarum strains; insight into how bacteria contribute to immunity in healthy individuals consuming the NSAID indomethacin

Paul de Vos, Zlatan Mujagic, Bart J de Haan, Roland J Siezen, Peter A Bron, Marjolein Meijerink, Jerry M Wells, Ad AM Masclee, Mark Boekschoten, Marijke M Faas, Freddy J Troost

\section{Submitted}




\section{Abstract}

\section{Objective}

Probiotic consumption is known to impact host immunity, however, the mechanisms involved and how bacteria are perceived in healthy adults under commonly encountered mucosal enteropathies, such as consumption of NSAID-painkillers, remain unclear.

\section{Methods}

Adults consuming indomethacin (a NSAID) received three different strains of Lactobacillus plantarum bacteria (TIFN101, CIP48, or WCFS1) or placebo. To gain insight into mechanisms, CD45RO+ memory cells were challenged with either superantigen (total responsiveness), tetanus-toxoid (specific pathogen responses), or bacterial cell wall components (responses against the bacterium).

\section{Results}

The NSAID induced a reduction in serum $\mathrm{CD}^{+} /$Foxp3 regulatory cells, which was prevented by TIFN101. Cell wall components provoked a response after WCFS1 and TIFN101 consumption. In addition TIFN101 also enhanced specific responses against tetanus toxoid-antigen. In the mucosa of the human proximal small intestine, TIFN101 upregulated expression ofgenes associated with maintenance of $\mathrm{T}$ and $\mathrm{B}$-cell function and antigen-presentation. CIP48 and WCFS1 downregulated immunological pathways involved in antigen-presentation and shared downregulation of snoRNAs suggesting cellular destabilization. Full sequencing of the strains revealed that TIFN101 lacks the genes requiredfor plantaricin biosynthesis as well as for exopolysaccharide biosynthesis, many sugar utilization cassettes, and two large LPXTG-anchored mucusbinding proteins.

\section{Conclusion}

The impact of $L$. plantarum intake on immunity is strain dependent and involves responses against cell wall components as well as induction of specific responses against pathogens by enhancing antigen presentation and leucocyte maintenance in the mucosa. Effects of $L$. plantarum intake in healthy humans consuming NSAIDs were positive for TIFN101 consumption; while evidence was found that CIP48 and WCFS1 destabilized intestinal repair. 


\section{Introduction}

Commensal Lactobacilli species may play an active role in the maintenance of homeostasis of intestinal immune responses by affecting the populations and distribution of immune cells. ${ }^{1-5}$ Lactobacilli likely regulate immune cells via the interaction of bacterial cell-wall components or secreted bacterial products with immune or epithelial cells in the human mucosa. ${ }^{4,6,7}$ These interactions, however, seem to contribute to more than just tolerance to the beneficial microbes. Lactobacilli have been shown to have a positive impact on immune responses, as demonstrated in several vaccination studies. ${ }^{8-14}$ Also, Lactobacilli have been shown to activate more tolerogenic cellular pathways in human intestinal mucosal cells. ${ }^{15,16}$

Lactobacillus plantarum is a well-characterized member of the dietary group of lactic acid bacteria, having a "generally recognized as safe" (GRAS) status. ${ }^{17}$ In a previous study, we showed that $L$. plantarum strains had different effects on human dendritic cells and human peripheral blood mononuclear cells. ${ }^{6,7}$ Using comparative genomic hybridization, we identified a number of bacteriocins and cell-wall components involved in the glycosylation of cell wall teichoic acids associated with these differential effects. ${ }^{4,6}$ We assumed that the differential expression of $L$. plantarum genes contributes to the observed differences in activation of Toll-like receptor (TLR) 2-4 and CD14 antigens in the host.[6] As a consequence of differences in TLR-binding, upon incubation with dendritic cells $L$. plantarum-strains induce alterations in production of the pro-inflammatory cytokine IL-12 and the regulatory cytokine IL-10. ${ }^{6}$ On the basis of these findings three strains, L. plantarum WCFS1 (WCFS), L. plantarum CIP104448 (CIP48), and L. plantarum TIFN101 (TIFN101) were selected, which induced IL-10/IL-12 ratios in vitro classified as proinflammatory, neutral, or anti-inflammatory, respectively. In this study, we investigate human in vivo immune responses after consumption of the three L. plantarum-strains. Healthy volunteers received the non-steroidal antiinflammatory drug (NSAID) indomethacin, which induces mild and reversible damage to the gastrointestinal lining. ${ }^{18-21}$ In western societies millions of individuals take NSAIDs for the treatment of among others musculoskeletal pain. The unwanted mucosal effects of indomethacin are thought to be caused by inhibition of cyclooxygenase 1 (COX-1) resulting in decreased production of prostaglandin (PG) E2 and temporary disruption of epithelial integrity. ${ }^{21-25}$ Polarized T-cell responses in the peripheral circulation, as well as the responses after re-stimulation with cell wall extract of $L$. plantarum-strains, superantigen, or tetanus were also studied to determine whether the $L$. plantarum strains could enhance immunity against one of these stimuli. Mucosal transcriptional responses were studied in vivo in the duodenum after seven days of daily consumption of one of the three L. plantarum strains using a randomized doubleblind placebo-controlled cross-over design. Furthermore, the probiotic strains were sequenced and annotated to identify putative gene clusters associated with the differential responses induced by the probiotics. 


\section{Materials and methods}

\section{Bacterial strains and growth conditions}

L. plantarum WCFS1, ${ }^{26}$ L. plantarum CIP104448, and L. plantarum TIFN101 (NIZO straincollection) were cultured at $37^{\circ} \mathrm{C}$ in Man, Rogosa, and Sharpe (MRS) medium (Merck). Detailed protocols for culturing, harvesting, freeze drying, storing, and viability determination of Lactobacillus species has been published. ${ }^{2}$ Maltodextrin and glucose were added to a final concentration of $20 \%$ and $2 \%$ (wt/vol), respectively, to obtain bacterial preparations (WCFS, $2.6 \times 10^{9} \mathrm{CFU}$; CIP48, $2.4 \times 10^{9} \mathrm{CFU}$; and TIFN101, $5.6 \mathrm{x}$ $10^{9} \mathrm{CFU}$ ); placebo controls contained the two sugars.

\section{Volunteers and interventions}

This study was approved by the University Hospital Maastricht Ethical Committee and has been registered in the US National Library of Medicine (http://www.clinicaltrials.gov, NCT01456767). All subjects gave their written informed consent. Ten healthy volunteers, 7 female and 3 male $(26.3 \pm 10.1$ years, BMI of $21.8 \pm 2.40 \mathrm{~kg} / \mathrm{m}^{2}$ ), without a history of gastrointestinal symptoms and free of medication, were tested on four separate occasions (September 2013 till March 2014) in a randomized placebo-controlled cross-over study. The volunteers consumed their habitual diet and kept a gastrointestinal symptoms diary. Three days before the intervention, a blood sample was taken to obtain baseline values. The night before the starting day the volunteers ingested $75 \mathrm{mg}$ of indomethacin. On the start day the volunteers ingested another dosage of $50 \mathrm{mg}$ indomethacin to conform to a previously established protocol to establish mild gastrointestinal stress. ${ }^{27,28}$ Subsequently, the volunteers consumed the bacterium or placebo supplements for a period of seven days during lunch and during dinner. The vials containing bacteria or placebo were nontransparent. On the seventh day, tissue samples were obtained from the horizontal part of the duodenum by standard flexible gastroduodenoscopy at approximately $15 \mathrm{~cm}$ distal to the pylorus. The duodenal mucosa was chosen as the duodenum is readily accessible for sampling. The interventions were performed with an interval of four weeks to allow a wash-out period and also healing of the biopsy-sampling region.

\section{Cell staining}

Blood was collected in EDTA-containing tubes and processed for FACS analysis (Table 4.1). Isotype controls were used at the same dilution as the antibody. 
Table 4.1 Antibodies used for flow cytometry

\begin{tabular}{llll}
\hline Specificity & Clone Name & Fluorochrome & Supplier \\
\hline CD3 & UCHT1 & Pacific Blue & BD Pharmingen \\
CD8 & SK1 & PerCP & BioLegend \\
CD69 & FN50 & PE-Cy7 & BD Pharmingen \\
FoxP3 & 206D & APC & eBioscience \\
CD45RO & UCHL1 & Biotin + streptavidin PerCP & BioLegend \\
IL4 & MP4-25D2 & PE-Cy7 & BioLegend \\
IL-17A & eBio64DEC17 & Alexa488 & eBioscience \\
IL-21 & eBio3A3-N2 & PE & eBioscience \\
IFNy & B27 & Alexa700 & BD Pharmingen \\
CD56 & MEM-188 & APC & eBioscience \\
CD16 & CB16 & EFluor450 & eBioscience \\
CD335 & 9E2 & PE & BioLegend \\
CD161 & HP-3G10 & PE-Cy7 & eBioscience \\
\hline
\end{tabular}

To study T-cell polarization, $200 \mu$ of blood was diluted with $200 \mu$ l of RPMI1640 supplemented with heat-inactivated $10 \%$ foetal calf serum (FCS) and incubated with either phorbol-myristate-acetate (PMA; 80 nM Sigma-Aldrich, Steinheim, Germany) and 2nM calcium ionophore (Ca-lo; Sigma-Aldrich)(4 hr), Staphylococcus aureus-enterotoxin B (SEB) (5 $\mu \mathrm{g} / \mathrm{mL}$ Sigma, Deisenhofen, Germany) (24 hr), tetanus-toxoid (TT; $1.5 \mathrm{Lf} / \mathrm{mL}$, RIVM, Bilthoven, The Netherlands) (24 hr), or bacterial lysates $(30 \mu \mathrm{g} / \mathrm{mL})(24 \mathrm{hr})$. Stimulation with bacterial lysates was performed one week after treatment with one of the three strains. After stimulation, red blood cells were lysed with ammonium chloride, washed (PBS with $2 \%$ FCS), and incubated with different antibody-cocktails.

To stain for T-cells and T-cell subsets, cells were incubated with an antibody cocktail consisting of anti-CD3, anti-CD8, and anti-CD45RO for 30 minutes in the dark on ice. After washing with buffer, cells were incubated with streptavidin-Pacific Orange (1:100 Invitrogen) for 15 minutes on ice. After washing and centrifugation, pelleted cells were resuspended in Fix/Perm solution (eBioscience) for 45 minutes on ice. After washing in Perm-solution cells were incubated in mouse-serum for 15 minutes, followed by incubation with the cytokine antibody mix (anti-IL-4, anti-IFNy, anti-IL-17, and anti-IL21) or an isotype control mix for 30 minutes on ice. Cells were then washed with Perm-solution ( 3 times), resuspended in wash-buffer and measured by flowcytometry.

For staining NK-cells, cells were incubated with an antibody-cocktail consisting of antiCD3, anti-CD16, anti-CD56, anti-CD335, and anti-CD161 (NK cell staining), or with an isotype control cocktail for NK-cells consisting of anti-CD3, anti-CD16, anti-CD56, and isotype controls for anti-CD335 and CD161 for 30 minutes in the dark on ice. After washing with washing buffer, cells were fixed in FACS-lysis solution (BD Biosciences) for 30 minutes on ice, washed and analyzed by flow-cytometry. 


\section{RNA isolation and microarray}

Total RNA was isolated from duodenal biopsy samples using Trizol reagent $(1 \mathrm{ml})$ (Invitrogen, Breda, NL). RNA was purified using the Qiagen RNeasy Micro kit (Qiagen, $\mathrm{NL}$ ) and quantified using a NanoDrop ND-1000 spectrophotometer (Isogen Life Science, NL). RNA quality was confirmed using an Agilent 2100 bioanalyzer (Agilent Technologies, NL). RNA was judged suitable for array-hybridization only if samples exhibited intact bands corresponding to $18 \mathrm{~S}$ and $28 \mathrm{~S}$ ribosomal subunits and displayed no chromosomal peaks or RNA-degradation products.

Total RNA (100 ng) was used for whole transcript cDNA synthesis using the Ambion WT expression kit (Life Technologies, NL) and was subsequently labelled using the Affymetrix GeneChip WT Terminal Labeling Kit (Affymetrix, Santa Clara, CA). Samples were hybridized to human whole genome Affymetrix GeneChip Human Gene 1.1 ST arrays, washed, stained, and scanned on an Affymetrix GeneTitan instrument. Details on array handling can be found in the Affymetrix GeneTitan Instrument User Guide for Expression Array Plates (P/N 702933 Rev.2).

\section{Microarray data analysis}

Microarray analysis was performed utilizing MADMAX for statistical analysis. ${ }^{29}$ All arrays met the quality criteria. The probes on the Human Gene $1.1 \mathrm{ST}$-arrays were redefined according to Dai et al. ${ }^{30}$ based on the NCBI Entrez database (CDF version 15.1). In this way the Human Gene 1.1 ST array targets 19,682 unique genes. Normalized expression values were obtained from the raw intensity values by using the robust multi-array analysis preprocessing algorithm available in the AffyPLM library using default settings. ${ }^{31}$ Microarray-data were filtered and probe sets with at least five probes and expression values higher than 20 on at least five arrays with an interquartile range $>0.2$ (log2 scale) across all samples were selected for further statistical analysis. In addition, an InterQuartile Range (IQR) cut-off of 0.2 was used to filter out genes that showed no variation between the conditions. Differentially expressed genes were identified using linear models, applying moderated t-statistics that implemented empirical Bayes regularization of standard errors in the library limma. ${ }^{32}$ The moderated t-statistic was extended by a Bayesian hierarchical model to define an intensity-based moderated t-statistic to adjust for independence of variances relative to the degree of identity and relation between variance and signal intensity. ${ }^{33}$ Genes were defined as significantly changed when the $p$-value was $<0.05$ for pairwise comparisons.

\section{Pathway analysis}

Gene set enrichment analysis (GSEA; broad.mit.edu/gsea/) was performed using MADMAX and gene sets with a false discovery rate (FDR) $<0.2$ were considered significantly enriched. GSEA takes into account the broader context in which gene products function, namely in physically interacting networks such as biochemical, 
metabolic, or signal transduction routes, and has the advantage that it is unbiased because no gene selection step is used. Possible transcription factors were identified using Upstream Regulator Analysis in Ingenuity Pathway Analysis (IPA; Ingenuity Systems, Redwood City, CA).

\section{Genome sequencing and annotation}

For DNA preparation, bacteria were pelleted, washed, and resuspended in TES buffer (N-[tris(hydroxymethyl)methyl]-2-aminoet hanesulfonic acid). Cells were lysed with lysozyme $(360 \mathrm{mg} / \mathrm{ml})$ and mutanolysin $(140 \mathrm{U} / \mathrm{ml})$ by incubation for $2 \mathrm{~h}$ at $37^{\circ} \mathrm{C}$. Subsequently $300 \mu \mathrm{l}$ water and $80 \mu \mathrm{l}$ of a $20 \%$ SDS solution were added. The DNA extraction was done using phenol/chloroform (3x). DNA was precipitated with isopropanol and washed with $70 \%$ ethanol. Samples were treated with $100 \mu \mathrm{g} / \mathrm{ml}$ RNAse (Sigma) for one hour at $37^{\circ} \mathrm{C}$. DNA paired-end libraries with barcoding were made and sequenced using Illumina technology (Baseclear Leiden). The genome sequences of L. plantarum strains CIP104448 and TIFN 101 have been deposited in NCBI/GenBank under accession numbers JSUW00000000 and JSUX00000000, respectively. The contig sequences were submitted to the RAST automatic annotation server (http://rast.nmpdr.org/), which provided ORF calling and automatic annotation. The annotated contigs of CIP48 and TIFN101 were ordered by comparing them to the circular template genome of $L$. plantarum WCFS1, ${ }^{34}$ and comparing them to each other. Contigs/genes which did not match to the WCFS1 genome were annotated in more detail using BLASTP (http://blast.ncbi.nlm.nih.gov/) and InterProscan (http://www.ebi.ac.uk/interpro/). Ortholog groups (OGs) in the three genomes were determined using OrthoMCL (www.orthomcl.org/).

\section{Statistics}

Flow-cytometry results are expressed as the mean \pm standard error of the mean (SEM). Normal distribution was confirmed by the Kolmogorov-Smirnov test. The two-sided students t-test was used for changes in immune-cell populations after $L$. plantarum treatment. Gene expression data are depicted as the median (range). The two-sided Mann Whitney U-test was used to determine changes in expression-profiles after L. plantarum treatment in vivo. P-values $<0.05$ were considered statistically significant.

\section{Results}

\section{Human trial}

None of the volunteers experienced any discomfort during or after the seven day consumption period. Before the start of the trial (day 0) and again at day 7, blood 
samples were taken to study the effect of L. plantarum consumption on T-cell polarization. Also at day 7, duodenal biopsies were taken. Total RNA was isolated and hybridized to whole-genome expression microarrays.

\section{Cell frequencies and T-cell polarization after 6 days of treatment with L. plantarum}

We did not observe differences in the frequencies of total $\mathrm{CD}^{+}$cells, $\mathrm{CD}^{+} \mathrm{CD}^{+}$cells (naïve or memory), or the $\mathrm{CD}^{+} \mathrm{CD}^{+}$activated memory cells after treatment with any of the $L$. plantarum strains. However, the percentage of $\mathrm{CD} 4^{+}$Foxp $3^{+}$cells was significantly decreased following placebo and CIP48 treatment, but not after TIFN101 treatment (Figure 4.1A). Moreover, although we did not observe any effect with indomethacin treatment on $\mathrm{CD}^{+} \mathrm{CD}^{+}$(naïve and memory) cells, activated memory-cells exhibited a statistically significantly decrease after CIP48 treatment $(p<0.05)$ (Figure 4.1B).

Treatment did not influence percentages of NK-cells or NKT-cells. There was also no change in the percentages of the NK-cell subtypes (i.e. CD56 ${ }^{\text {high }}$ and $C D 56^{\text {dim }}$ ); while the expression of CD161 (KLRB1), mediating cytotoxicity, ${ }^{35,36}$ was also not affected by the $L$. plantarum treatments (results not shown).

T-cell polarization was studied after three types of T-cell stimulation: (i) non-specific polyclonal stimulation with $\mathrm{PMA} / \mathrm{Ca}^{2+}$ or superantigen (SEB) to study whether the total responsiveness was influenced by $L$. plantarum treatment, (ii) stimulation with a previously administered vaccine-antigen (tetanus toxoid (TT)) to study stimulation of specific memory responses, and (iii) stimulation with cell extracts of the specific $L$. plantarum strains in order to investigate whether specific immune responses against the $L$. plantarum were stimulated.

After non-specific stimulation with PMA/Ca-ionophore or SEB, we studied the percentage of IFNY, IL-4, II-17, or IL-21 positive Th cells and memory Th cells. Treatment with placebo or the administered $L$. plantarum strains did not influence cytokine production of the total population of Th cells or of Th memory cells after non-specific stimulation with PMA/Ca-ionophore. Although no differences were found after SEBstimulation in cytokine production by the total Th-cell population after the three $L$. plantarum treatments (results not shown), we did observe differences in cytokine production of the Th-memory cells after $L$. plantarum treatment. After stimulation with SEB, we observed a decreased percentage of IL-17-producing activated memory Th cells following treatment with CIP48 and an increased percentage of IL-17 producing activated memory Th cells after treatment with TIFN101 (Figure 4.2). Moreover, the percentage of IFN $\mathrm{p}$-producing activated memory Th cells was also increased after TIFN101 treatment (Figure 4.2).

Treatment with $L$. plantarum strains also modulated cytokine production following a more specific stimulation by TT (Figure 4.3). We studied cytokine production of memory Th cells in order to study the effect of $L$. plantarum treatment on memory cells. After TIFN101 treatment, the percentage IL-17 and the percentage IFN- $\gamma$ - 
producing memory Th cells were significantly increased, while no effect on cytokine production by memory Th cells after TT-stimulation was observed with the other $L$. plantarum strains.

Finally, we stimulated human blood samples with cell extracts of the L. plantarum strain that they had consumed in the study (Figure 4.4). We observed that subjects, who were treated with WCFS1, showed a trend towards an increased IL-17 response after stimulation with WCFS1 cell-extracts. Other cytokines were not affected after this treatment. There were no differences in cytokine production in subjects treated with CIP48, when their blood samples were stimulated with CIP48 cell-extract. When subjects were treated with TIFN101, their activated memory cells exhibited increased IL-17 and IFN- $\gamma$-production following stimulation with TIFN101 cell-extract.

A
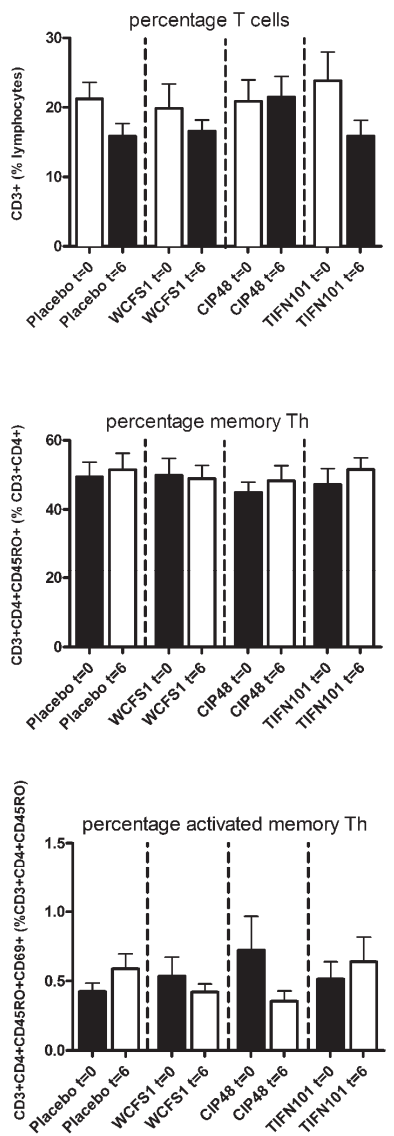
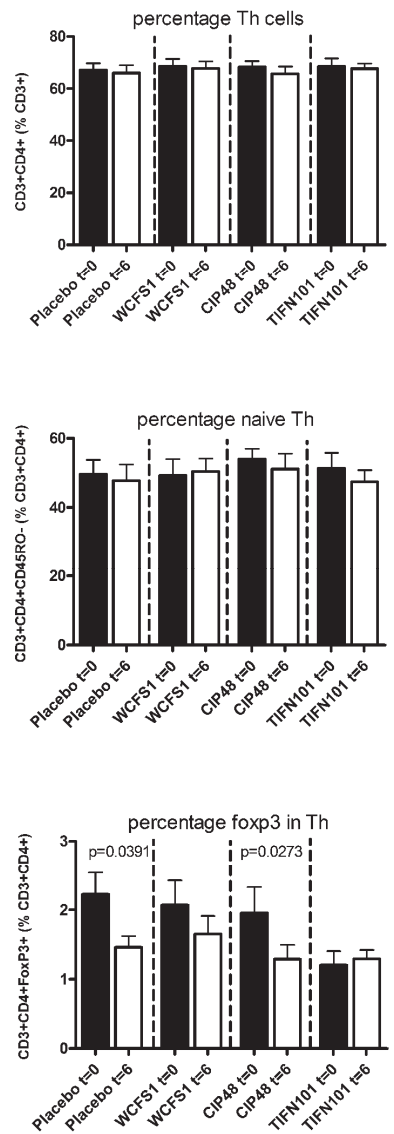
B
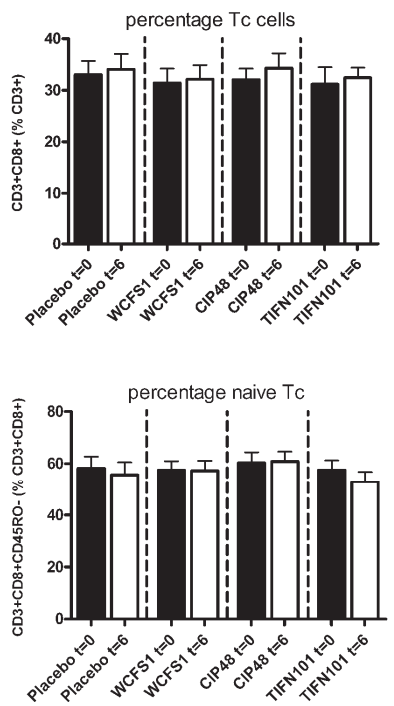
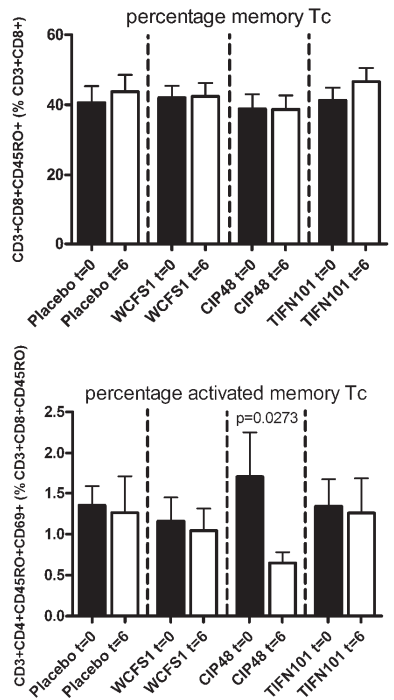

Figure 4.1 Effects of three L. plantarum strains on the frequency of $\mathrm{CD}^{+}(\mathrm{A})$ and $\mathrm{CD}^{+}(\mathrm{B}) \mathrm{T}$-cell populations in systemic circulation $(n=9)$. Statistical significance was calculated using the students t- test.
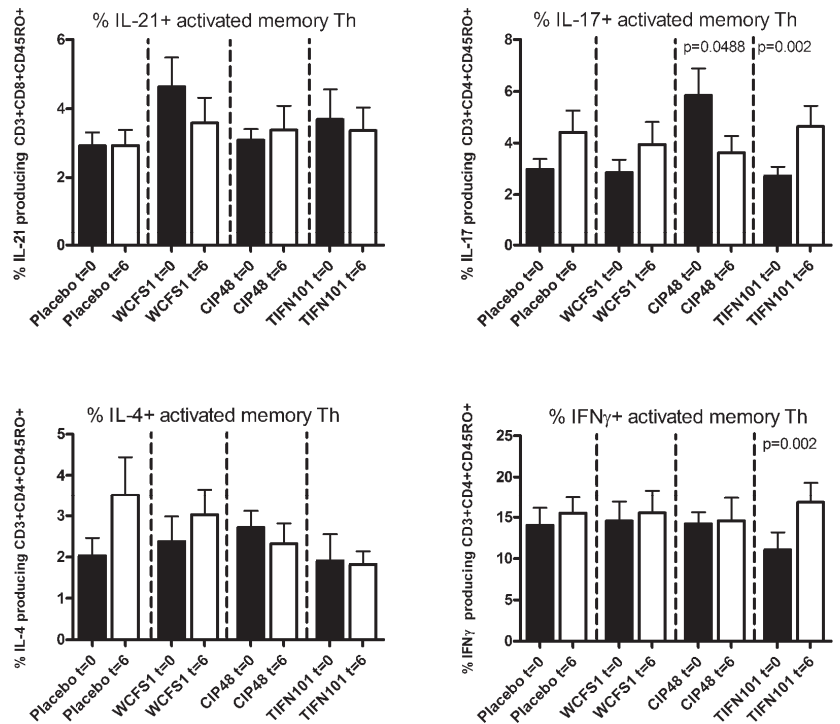

Figure 4.2 Effects of three L. plantarum strains on the frequency of IL-21, IL-17, IL-4, and IFN- - -producing Staphylococcus aureus enterotoxin B superantigen (SEB)-stimulated memory-CD45RO+ Th cells $(n=9)$. Statistical significance was calculated using the students $t$ - test. 

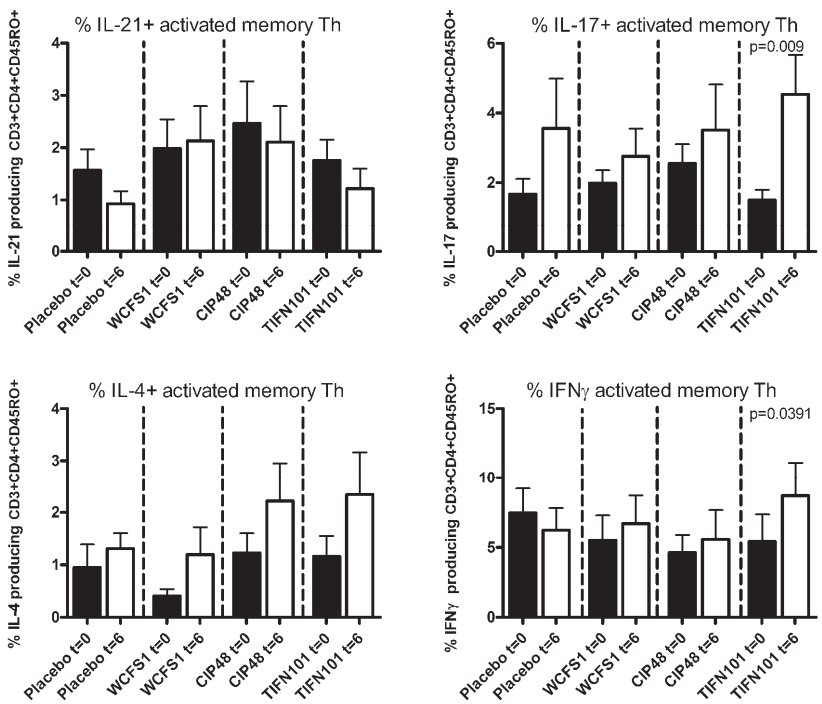

Figure 4.3 Effects of three L. plantarum strains on the frequency of IL-21, IL-17, IL-4, and IFN- $\nu$-producing tetanus-toxoid (TT)-stimulated memory-CD45RO+ Th cells $(n=9)$. Statistical significance was calculated using the students t- test.
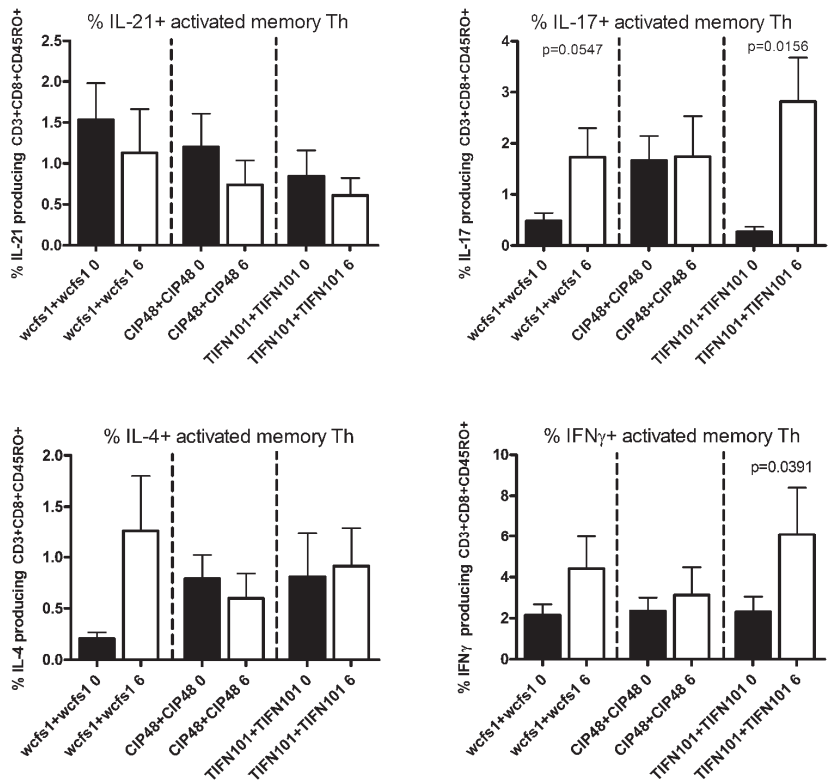

Figure 4.4 Effects of three $L$. plantarum strains on the frequency of IL-21, IL-17, IL-4, and IFN- $\gamma$-producing memory CD45RO+ Th cells stimulated with bacterial cell wall extracts, matched to the strain consumed $(n=9)$. Statistical significance was calculated using the students t- test. 


\section{Differential transcriptional response in duodenal mucosa upon exposure to the three L. plantarum strains}

Although we compared strains and not different bacterial species, we found unique gene expression profiles in the stressed intestines of the subjects consuming each bacterial strain. After treatment with the three strains, 315 genes were differentially regulated with L. plantarum WCFS1, 390 with CIP48, and 779 with TIFN101 (Figure 4.5A) as compared to the placebo intervention. Of these genes, WCFS1 shared only 35 upregulated genes with CIP48 and 9 with TIFN101 (Figure 4.5B). An additional 19 genes were downregulated when CIP48 was compared with WCFS1 and another 5 with TIFN101 (Figure 4.5C). Shared genes were mainly involved in general cellular functions and metabolism. As expected, in indomethacin-stressed intestine and also in placebo-treated controls, many processes associated with cellular repair were elevated. To gain more insight into the transcriptional changes induced by $L$. plantarum treatment, genes were subsequently ranked according to mean fold-change in expression. Table 4.2-4 lists the 10 genes most highly induced and 10 genes most downregulated. WCFS1 and CIP48 shared the downregulation of six small nucleolar RNAs (snoRNAs), i.e. snoRNA, (H)C/D(ACA) box 6, 14b, 53, 57, 60, 388. TIFN101 had a complete different profile of up and downregulation (Table 4.4). Of the most highly induced genes after TIFN101 consumption (the most immunological active $L$. plantarum), $80 \%$ are related to immunity:immunoglobulin lambda variable 6-57, putative V-set and immunoglobulin domain-containing protein 6-like, immunoglobulin lambda variable 7-46, interferon regulatory factor 4, GDNF family, CD27, CD79a, and plasminogen activator.

19,682 genes on the array

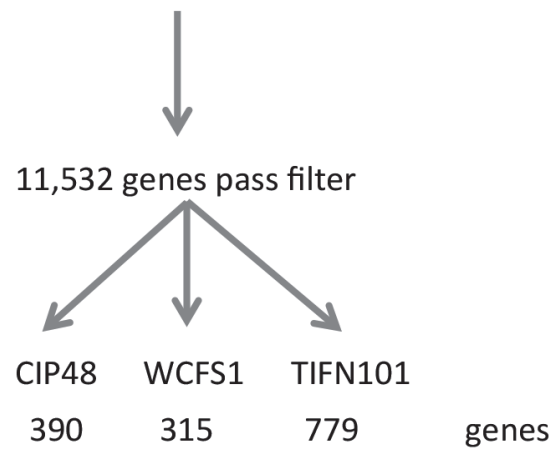

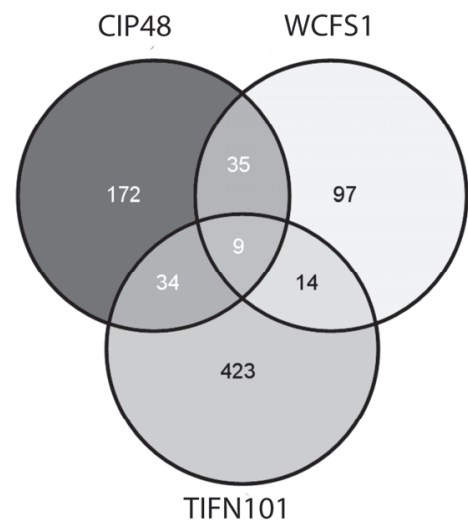

Figure 4.5 Flowchart of the microarray analysis $(A)$ and the number of unique genes regulated in human intestinal biopsies after consumption of three different L. plantarum strains (L. plantarum WCFS1 (WCFS1), L. plantarum CIP104448 (CIP48), L. plantarum TIFN101 (TIFN101)). Intensity > 20 on at least 5 arrays, interquartile range $>0.2$, at least 7 probes per gene. (B) Venn diagrams of the number of upregulated and $(C)$ downregulated genes in the intestinal biopsies after 
consumption of L. plantarum and indomethacin.

Table 4.2 The ten most up- and downregulated genes in NSAID-stressed human intestine after consumption of L. plantarum WCFS1 (WCSF1).

\begin{tabular}{lccc}
\hline Top 10: Upregulated genes placebo versus WCFS1 & Gene name & $\begin{array}{c}\text { IBMT } \\
\text { p-value }\end{array}$ & $\begin{array}{c}\text { Mean fold } \\
\text { versus control }\end{array}$ \\
\hline kinesin family member 20B & KIF20B & 0.01 & 1.34 \\
microRNA 186 & MIR186 & 0.03 & 1.31 \\
guanylate cyclase activator 2A (guanylin) & GUCA2A & 0.04 & 1.31 \\
integrin, alpha 4 (antigen CD49D, alpha 4 subunit of VLA-4 receptor) & ITGA4 & 0.01 & 1.31 \\
centromere protein E, 312kDa & CENPE & 0.05 & 1.30 \\
putative homeodomain transcription factor 1 & PHTF1 & 0.00 & 1.30 \\
spindle and kinetochore associated complex subunit 2 & SKA2 & 0.01 & 1.29 \\
killer cell lectin-like receptor subfamily D, member 1 & KLRD1 & 0.00 & 1.29 \\
gamma-aminobutyric acid (GABA) A receptor, alpha 2 & GABRA2 & 0.02 & 1.27 \\
retinitis pigmentosa GTPase regulator & RPGR & 0.01 & 1.27 \\
\hline Top 10: Downregulated genes placebo versus WCFS1 & & & \\
small nucleolar RNA, H/ACA box 16A & SNORA16A & 0.02 & -1.35 \\
small nucleolar RNA, C/D box 53 & SNORD53 & 0.04 & -1.36 \\
contactin 3 (plasmacytoma associated) & CNTN3 & 0.00 & -1.38 \\
small nucleolar RNA, C/D box 6 & SNORD6 & 0.04 & -1.39 \\
small nucleolar RNA, H/ACA box 57 & SNORA57 & 0.00 & -1.44 \\
small nucleolar RNA, H/ACA box 60 & SNORA60 & 0.00 & -1.44 \\
small nucleolar RNA, H/ACA box 14A & SNORA14A & 0.03 & -1.49 \\
small nucleolar RNA, H/ACA box 38B (retrotransposed) & SNORA38B & 0.01 & -1.58 \\
small nucleolar RNA, H/ACA box 14B & SNORA14B & 0.01 & -1.58 \\
small Cajal body-specific RNA 4 & SNORA16A & 0.01 & -1.72 \\
\hline
\end{tabular}

Table 4.3 The ten most up- and downregulated genes in NSAID-stressed human intestine after consumption of L. plantarum CIP104448 (CIP48).

\begin{tabular}{lccc}
\hline Top 10: Upregulated genes placebo versus CIP48 & Gene name & $\begin{array}{c}\text { IBMT } \\
\text { p-value }\end{array}$ & $\begin{array}{c}\text { Mean fold } \\
\text { versus control }\end{array}$ \\
\hline coiled-coil domain containing 59 & CCDC59 & 0.01 & 1.33 \\
aldehyde dehydrogenase 1 family, member L2 & ALDH1L2 & 0.03 & 1.32 \\
KIAA0125 & KIAA0125 & 0.00 & 1.31 \\
phospholipase C, beta 4 & PLCB4 & 0.01 & 1.31 \\
coiled-coil domain containing 102B & CCDC102B & 0.04 & 1.29 \\
RAS guanyl releasing protein 3 (calcium and DAG-regulated) & RASGRP3 & 0.03 & 1.28 \\
peptidase domain containing associated with muscle regeneration 1 & PAMR1 & 0.00 & 1.28 \\
DEP domain containing 1 & DEPDC1 & 0.01 & 1.28 \\
phospholipase A2, group IIA (platelets, synovial fluid) & PLA2G2A & 0.02 & 1.28 \\
heparan sulfate (glucosamine) 3-O-sulfotransferase 3B1 & HS3ST3B1 & 0.04 & 1.28 \\
\hline Top 10: Downregulated genes placebo versus CIP48 & & & \\
potassium channel, subfamily K, member 15 & KCNK15 & 0.05 & -1.33 \\
transient receptor potential cation channel, subfamily V, member 6 & TRPV6 & 0.01 & -1.33 \\
long intergenic non-protein coding RNA 282 & LINC00282 & 0.03 & -1.35 \\
ephrin-A1 & EFNA1 & 0.02 & -1.38 \\
matrix metallopeptidase 10 (stromelysin 2) & MMP10 & 0.05 & -1.41 \\
angiopoietin-like 4 & ANGPTL4 & 0.03 & -1.47 \\
heme oxygenase (decycling) 1 & HMOX1 & 0.00 & -1.50 \\
nuclear factor, interleukin 3 regulated & NFIL3 & 0.01 & -1.52 \\
major facilitator superfamily domain containing 2A & MFSD2A & 0.02 & -1.59 \\
glucose-6-phosphatase, catalytic subunit & G6PC & 0.02 & -1.65 \\
\hline
\end{tabular}


Table 4.4 The ten most up- and downregulated genes in NSAID-stressed human intestine after consumption of L. plantarum TIFN101 (TIFN101).

\begin{tabular}{lccc}
\hline Top 10: Upregulated genes placebo versus TIFN101 & Gene name & $\begin{array}{c}\text { IBMT } \\
\text { p-value }\end{array}$ & $\begin{array}{c}\text { Mean fold } \\
\text { versus control }\end{array}$ \\
\hline immunoglobulin lambda variable 6-57 & IGLV6-57 & 0.01 & 1.63 \\
putative V-set and immunoglobulin domain-containing protein 6-like & LOC642131 & 0.00 & 1.55 \\
immunoglobulin lambda variable 7-46 (gene/pseudogene) & IGLV7-46 & 0.04 & 1.48 \\
heparan sulfate (glucosamine) 3-O-sulfotransferase 3B1 & HS3ST3B1 & 0.00 & 1.41 \\
interferon regulatory factor 4 & IRF4 & 0.00 & 1.40 \\
GDNF family receptor alpha 2 & GFRA2 & 0.00 & 1.40 \\
CD27 molecule & CD27 & 0.01 & 1.40 \\
CD79a molecule, immunoglobulin-associated alpha & CD79A & 0.03 & 1.38 \\
plasminogen activator, tissue & PLAT & 0.00 & 1.37 \\
Der1-like domain family, member 3 & DERL3 & 0.00 & 1.37 \\
\hline Top 10: Downregulated genes placebo versus TIFN101 & & & -1.40 \\
small nucleolar RNA, H/ACA box 38B (retrotransposed) & SNORA38B & 0.04 & -1.40 \\
small nucleolar RNA, H/ACA box 21 & SNORA21 & 0.03 & -1.41 \\
small nucleolar RNA, H/ACA box 60 & SNORA60 & 0.01 & -1.43 \\
small nucleolar RNA, C/D box 53 & SNORD53 & 0.01 & -1.43 \\
ephrin-A1 & EFNA1 & 0.00 & -1.45 \\
small nucleolar RNA, C/D box 6 & SNORD6 & 0.02 & -1.46 \\
small nucleolar RNA, H/ACA box 57 & SNORA57 & 0.00 \\
small nucleolar RNA, H/ACA box 14B & SNORA14B & -1.47 \\
family with sequence similarity 5, member C & FAM5C & -1.73 \\
small Cajal body-Specific RNA 4 & SCARNA4 & 0.03 & -1.97
\end{tabular}

Based on the immune data, we expected to find differential changes in specific transcription factors. To identify these transcription factors and to identify pathways regulated by the different strains, Ingenuity Pathway Analysis (IPA) (Figure 4.6) was performed. As shown in Figure 4.6, TIFN101 induced more changes than CIP48 and WCSF1 in the NSAID-stressed intestine. The most significant set of target-genes in the TIFN101 group were immune response-related genes. TIFN101 upregulated MHC-lia, while downregulation of MHC-II $\beta$ was observed with CIP48 and WCFS1 (Figure 4.7). This might explain the enhanced responses to antigens such as TT in the TIFN101 treated group. Another pathway that might contribute to the enhanced responses in TIFN101 is the upregulation of genes involved in leucocyte extravasation (Figure 4.8). Interestingly, TIFN101 enhanced RAPL expression, which is a GTPase involved in regulating integrin affinity. Concomitantly, an upregulation of essential adhesion molecules such as ICAM-1 and Cadherin 5 (CDH-5) was seen, illustrating the upregulation of immune cell migration pathways by TIFN101. Also, some regulation of leukocyte extravasation was also observed with CIP48 and WCFS1; however, it was much less pronounced than in the TIFN101 group. 
Effects of L. plantarum on immunity
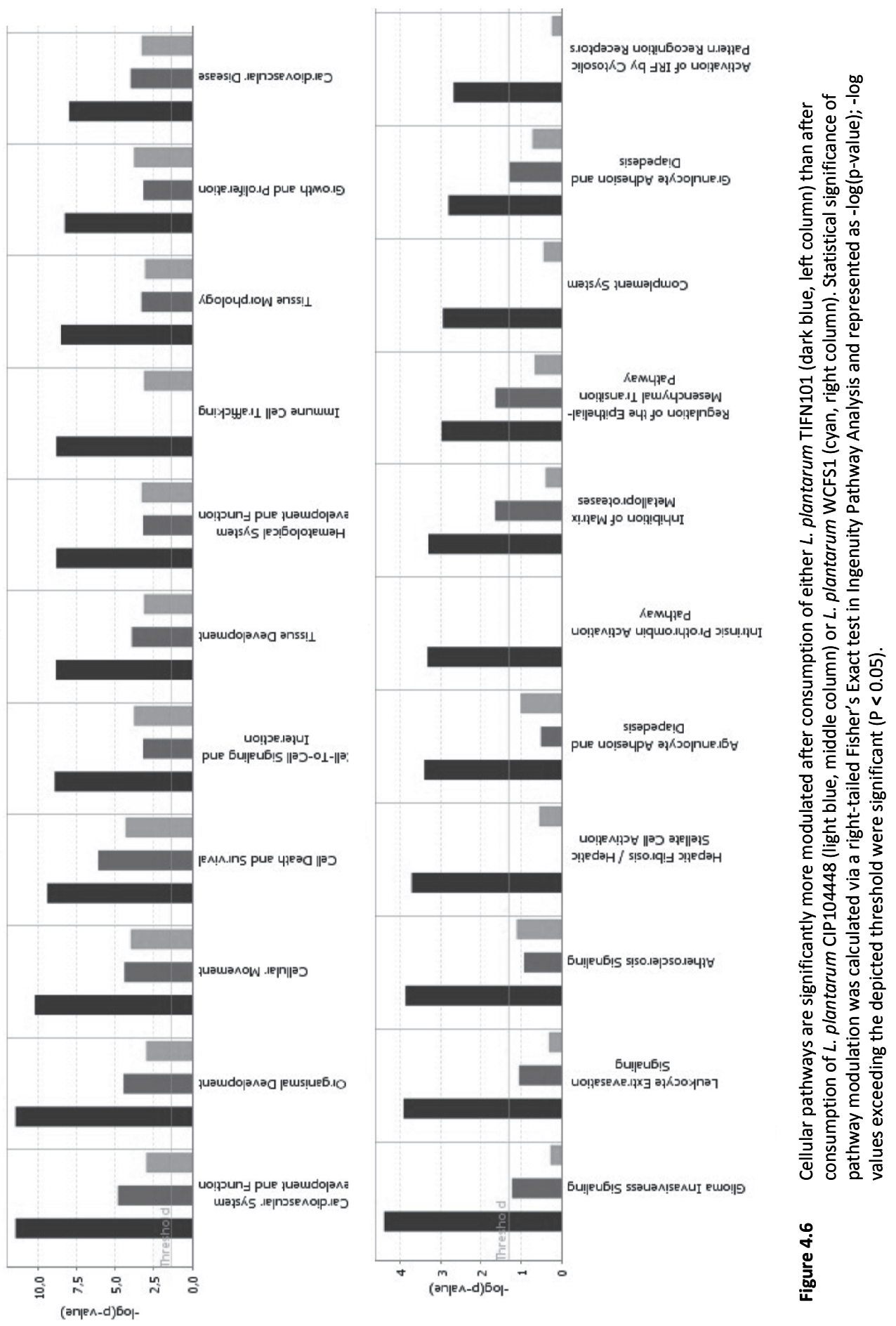


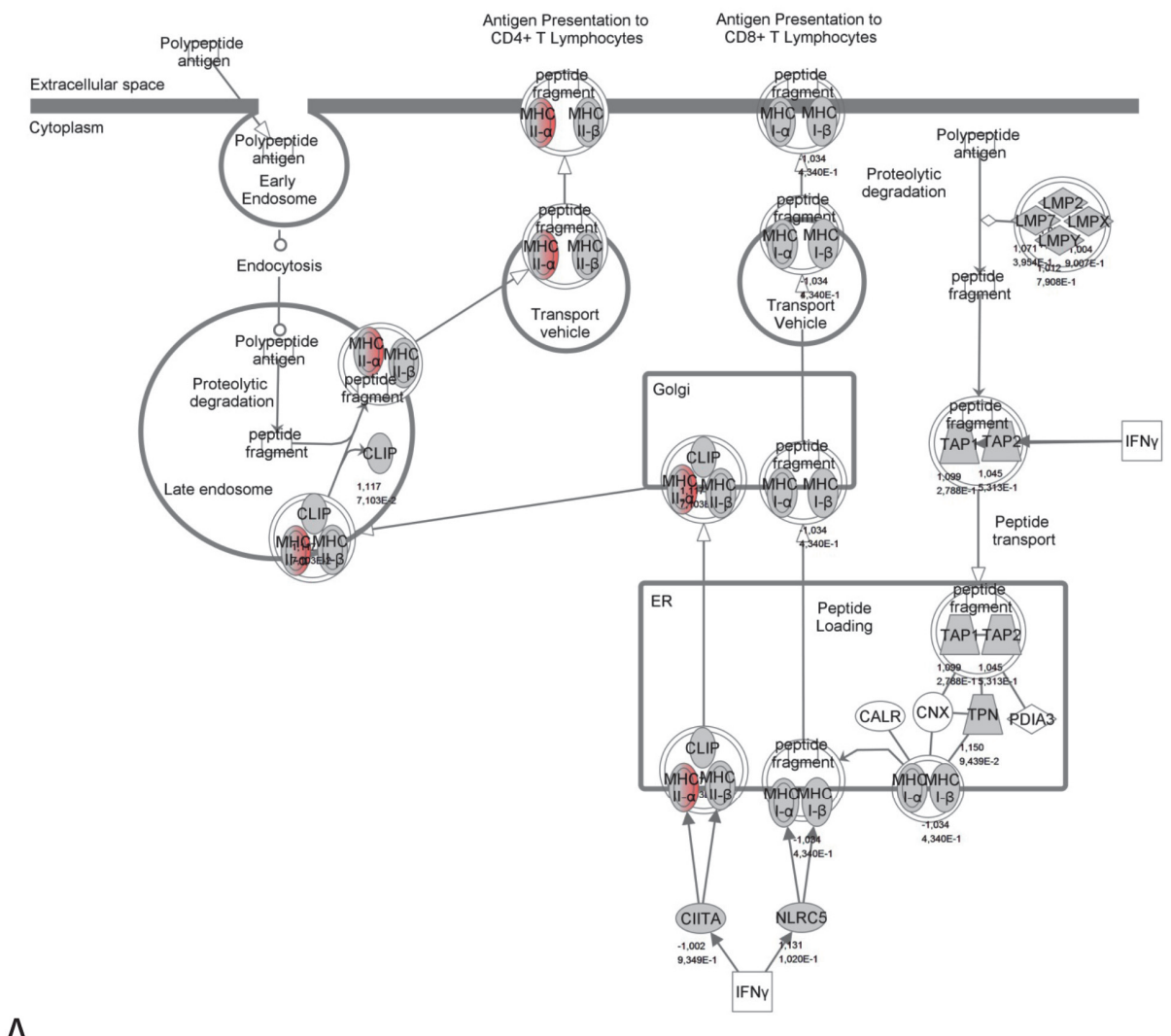

Figure 4.7A Regulation of genes involved in antigen presentation in NSAID-stressed intestine after consumption of L. plantarum TIFN101.

Red indicates upregulation while green depicts downregulation of the specific gene. 


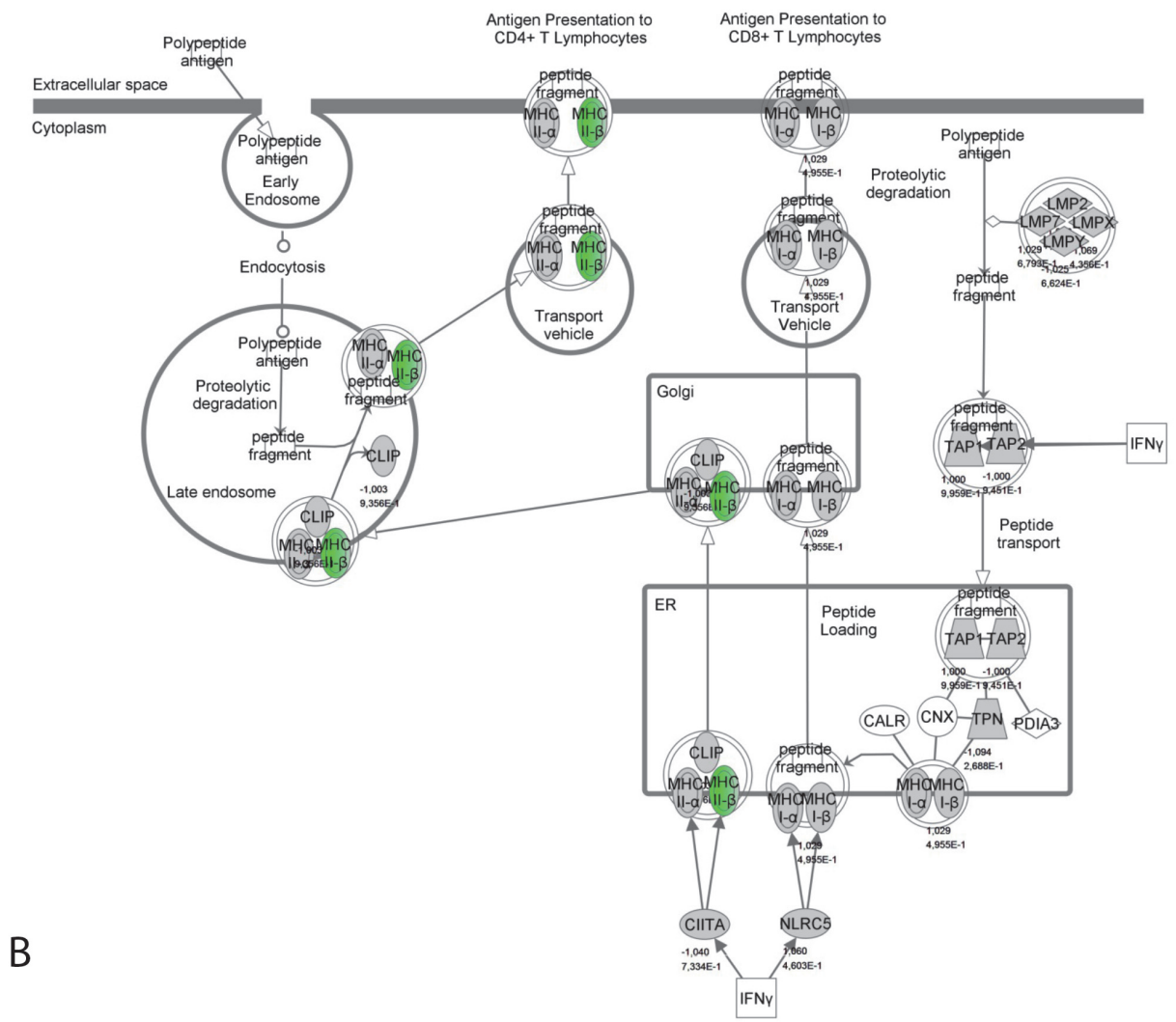

Figure 4.7B Regulation of genes involved in antigen presentation in NSAID-stressed intestine after consumption of L. plantarum CIP104448.

Red indicates upregulation while green depicts downregulation of the specific gene. 


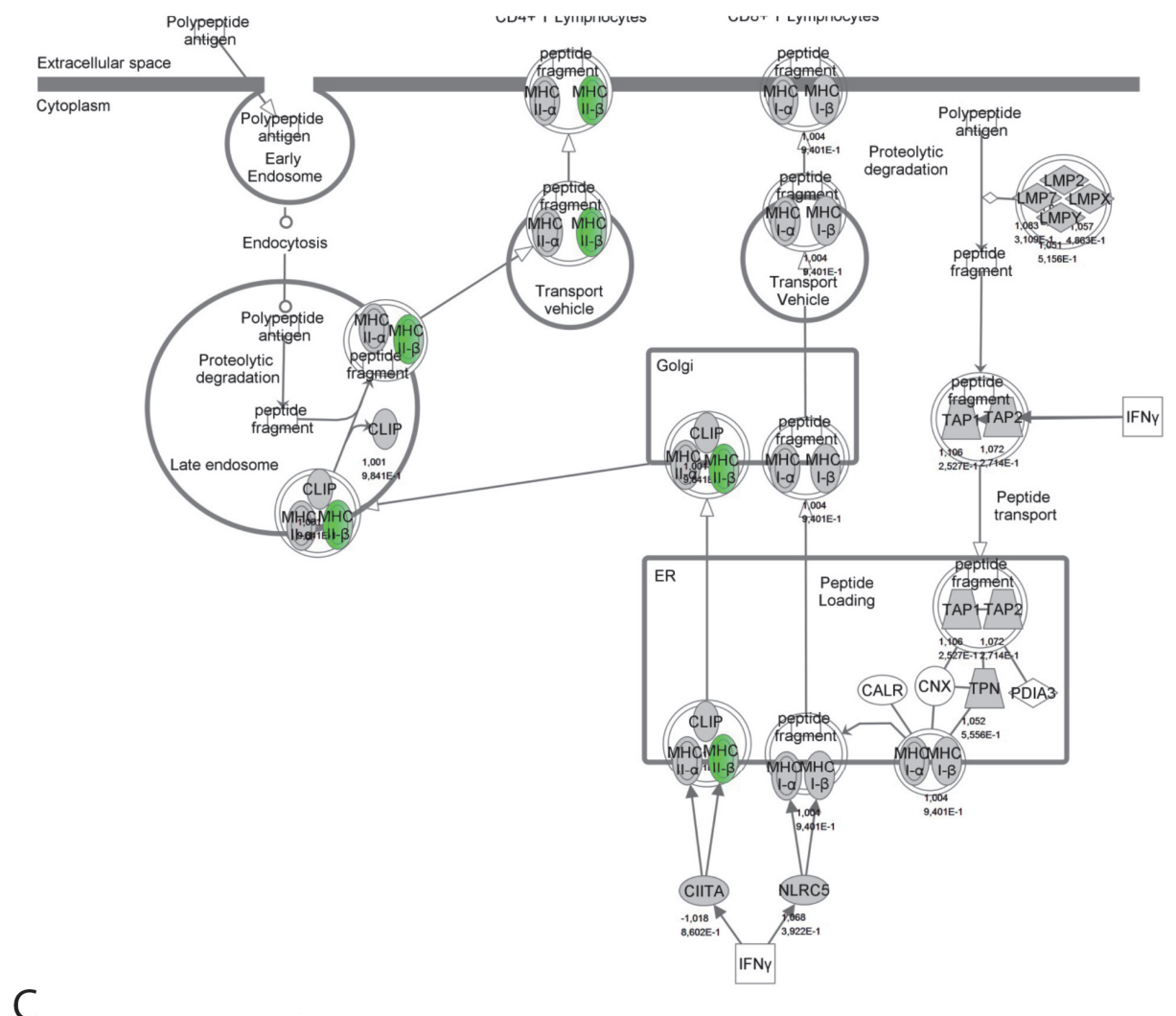

Figure 4.7C Regulation of genes involved in antigen presentation in NSAID-stressed intestine after consumption of $L$. plantarum WCFS1.

Red indicates upregulation while green depicts downregulation of the specific gene. 


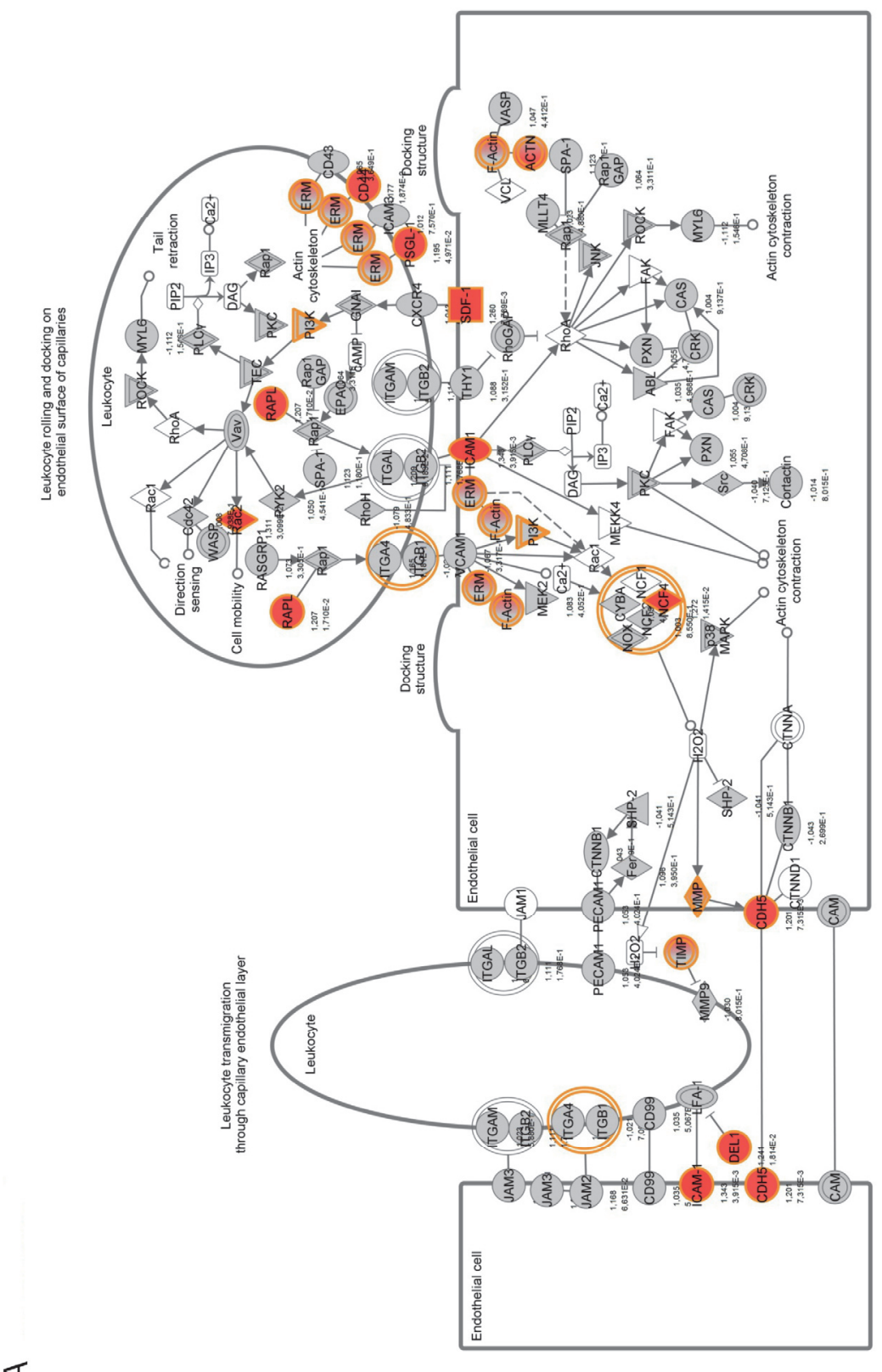

Figure 4.8A Regulation of genes involved in leukocyte extravasation pathways in NSAID-stressed intestine after consumption of $L$. plantarum TIFN101.

Red indicates upregulation while green depicts downregulation of the specific gene. 


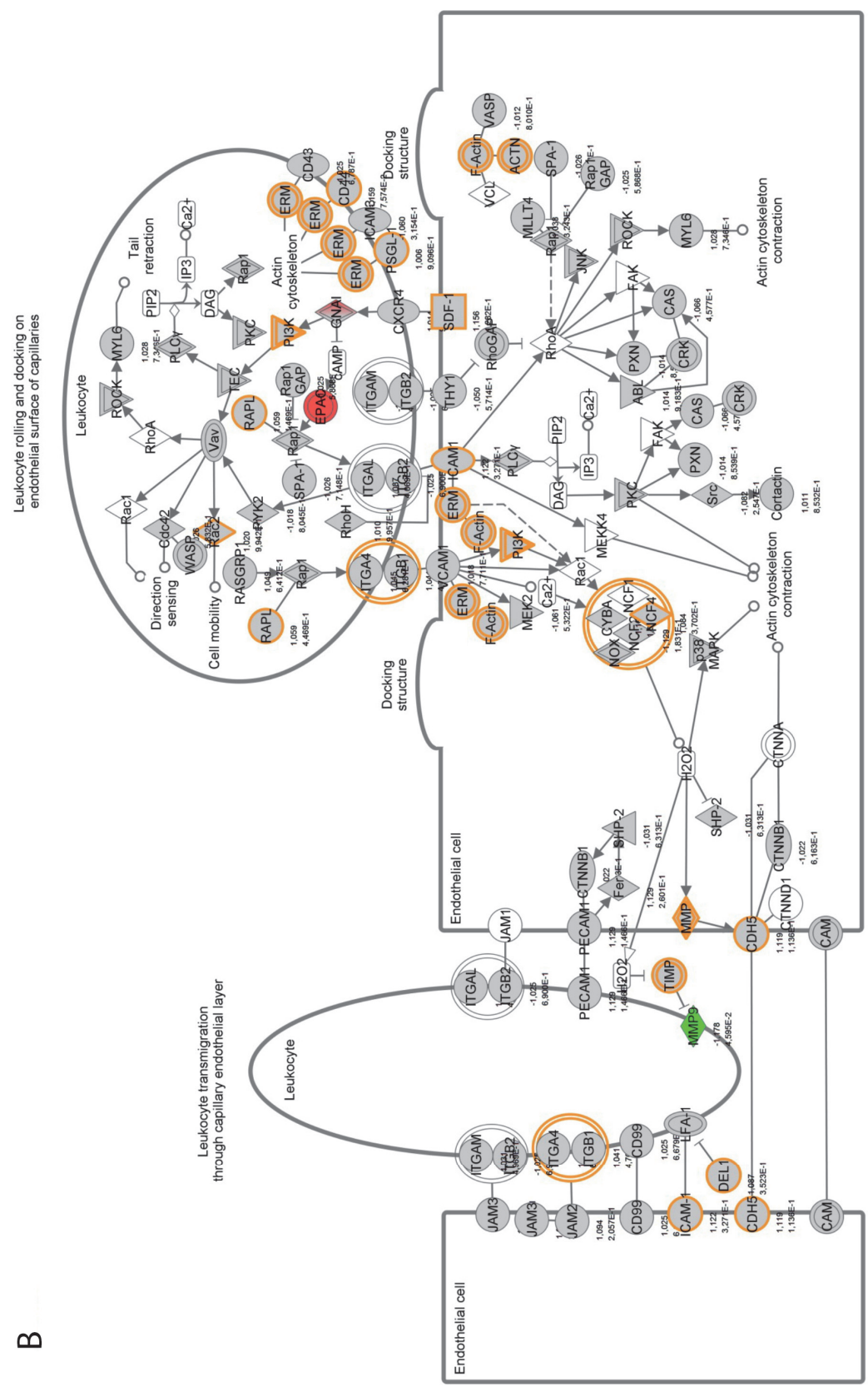

Figure 4.8B Regulation of genes involved in leukocyte extravasation pathways in NSAID-stressed intestine after consumption of L. plantarum CIP104448.

Red indicates upregulation while green depicts downregulation of the specific gene. 


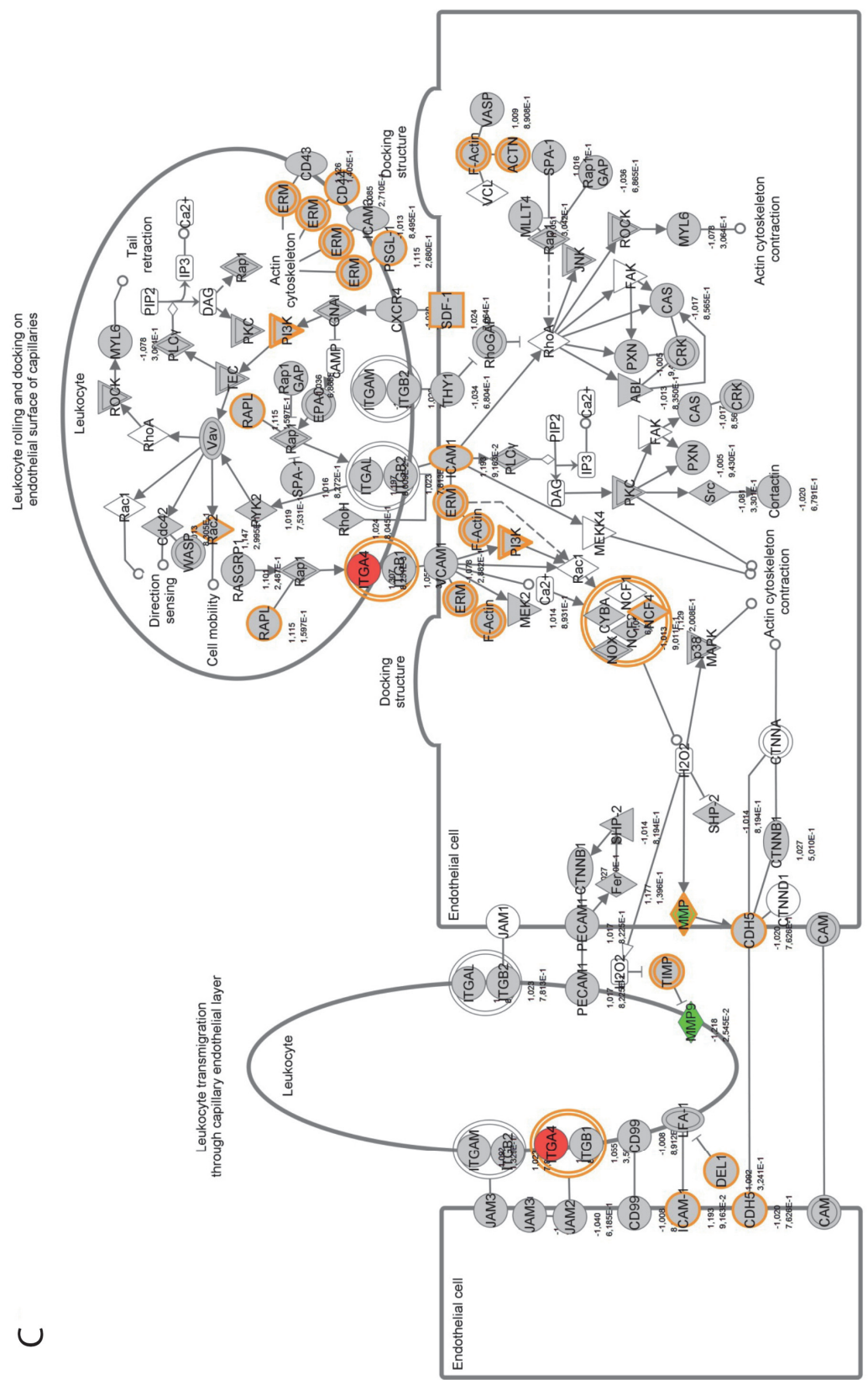

Figure 4.8C Regulation of genes involved in leukocyte extravasation pathways in NSAID-stressed intestine after consumption of L. plantarum WCFS1.

Red indicates upregulation while green depicts downregulation of the specific gene. 


\section{Differential gene expression profiles between the three L. plantarum strains}

The three L. plantarum strains, L. plantarum CIP104448, and L. plantarum TIFN101 were sequenced, annotated, and compared with the genome (chromosome and plasmids) of L. plantarum WCFS1. ${ }^{34}$ A total of 3010 ortholog groups (OGs) were assigned to the chromosome, based on the ordering of contigs to the template WCFS1 genome. The three genomes shared 2455 of the 3010 chromosomal OGs $(=81.5 \%)$, which is defined as the core genome for this study. When the contigs and OGs/genes are included that do not match to the WCFS1 chromosome, much higher numbers of unique genes are found for the CIP48 and TIFN101 genomes (Figure 4.9). Many of these extra unique genes are on plasmids (see Table 5, supplementary data). Tables 6 and 7 in the supplementary data list the unique genes for L. plantarum TIFN101 and $L$. plantarum CIP104448. Tables 4.8 and 4.9 in the supplementary data list genes that are absent in the two strains. The full analysis reveals that L. plantarum CIP104448 lacks the complete plantaricin biosynthesis gene cluster (and a large set of genes adjacent to this cluster (i.e. OGs 334-348) and the entire gene cluster for EPS biosynthesis. Also, we analyzed the genes that are unique for (Table 4.9a) or absent (Table 4.9b) in $L$. plantarum CIP104448 and L. plantarum TIFN101 as compared to L. plantarum WCFS1 as these are potential candidate genes for the biological effects of the two strains. Additionally, L. plantarum TIFN101 is missing some genes associated with plantaricin biosynthesis as well as genes for exopolysaccharide biosynthesis, many sugar utilization cassettes, and two large LPXTG-anchored mucus-binding proteins.

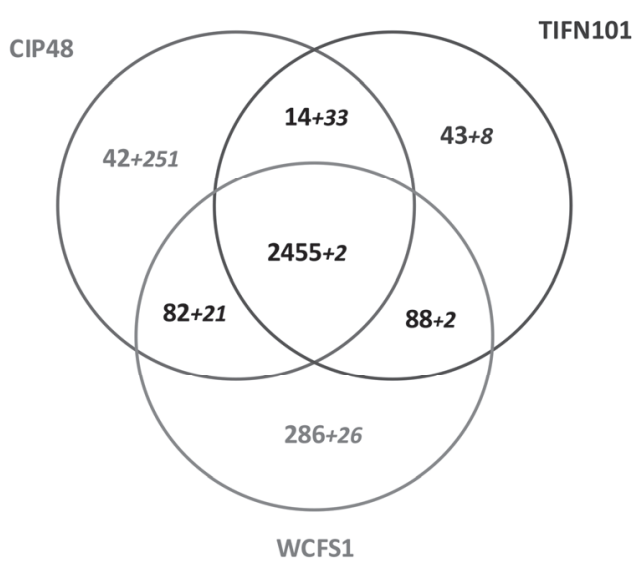

Figure 4.9 Venn diagram of shared and unique genes in all contigs of L. plantarum strains (L. plantarum WCFS1 (WCFS1), L. plantarum CIP104448 (CIP48), L. plantarum TIFN101 (TIFN101)). The smaller numbers in italics represent OGs that do not match to the chromosome of strain WCFS1. These numbers do not include the putative prophage genes in each genome. 


\section{Discussion}

This study was carried out to investigate whether L. plantarum strains selected in vitro for their differential immune stimulating capacity have different impacts on local and systemic immunity in healthy individuals undergoing a mild, commonly encountered stressor of intestinal immunity. All three strains had an effect on immunity, but the type of effect was highly strain-dependent and may not be beneficial in certain contexts. The immune responses induced by the strains in vivo were very different to that predicted in vitro. Consumption of NSAID induced a reduction in CD4+/Foxp3 regulatory cell-frequencies, which was prevented by WCFS1 and TIFN101 administration. This should be considered to be a beneficial regulatory effect. CIP48 did not prevent NSAID induced reduction of CD4+Foxp3 and had more negative effects. CIP48 reduced the number of memory cells suggesting a pro-inflammatory, and thereby implying negative effects of consumption of this bacterium.

T-cell polarization was studied after different stimuli to gain insight into the mechanisms by which bacteria might influence immunity. The hypothesis was that bacterial cell-wall components might induce immune responses ${ }^{2}$ and enhance systemic immunity as a bystander effect. This happened with WCFS1 and TIFN101 as shown in Figure 4.4. However, the most pronounced stimulator of immunity, i.e. TIFN101, also showed enhanced responses against specific pathogenic antigens such as TT and had an overall enhanced response to the SEB challenge. It is unlikely that the enhanced response against TT in the TIFN101 consumers is caused solely by the response against TIFN101 cell-wall components. This is supported by the analysis of the intestinal biopsies that suggest that TIFN101 stimulate specific processes in the intestine.

Analysis of the mucosal transcriptome suggests that TIFN101, in contrast to the other strains, upregulates processes associated with $\mathrm{T}$ - and B-cell function and antigen presentation. It also had a pronounced effect on CD27 upregulation, which is required for generation and long-term maintenance of T cell immunity. ${ }^{37}$ TIFN101 also enhanced expression of MHC-Il $\alpha$ in mucosa as well as key regulatory molecules such as RAPL. RAPL enhances integrin affinity and the adhesion of T-cells. ${ }^{38,39}$ These observations in the mucosa may explain the enhanced memory T-cell responses observed with TIFN101 consumption. Furthermore, B-cell immunity in the mucosa was enhanced as illustrated by upregulation of immunoglobulin regulatory genes and by CD79a. CD79a is also known as B-cell antigen receptor complex-associated protein alpha-chain forming, together with $\mathrm{CD} 79 \mathrm{~b}$ protein, the B-cell antigen receptor complex-associated protein. ${ }^{40}$ CIP48 and WCFS1 did not have these effects; in fact, they had a tendency to downregulate processes such as antigen presentation in the mucosa.

To our knowledge, this is the first report that bacteria can downregulate snoRNAs in the stressed intestine. SnoRNA are metabolically stable, non-coding RNAs that associate with a set of proteins to form small nucleolar RNPs (snoRNPs). The majority of snoRNAs function to guide RNAs in the post-transcriptional synthesis of 
2'-O-methylated nucleotides and pseudo-uridines in rRNAs, small nuclear RNAs (snRNAs), and other cellular RNAs including mRNAs. ${ }^{41-43}$ The relative reduction in snoRNAs 53, 57, 60 by CIP48 and WCFS1 suggest a downregulation of methylation of ribosomal $\mathrm{RNA}^{44}$ and downregulation of $14 \mathrm{~b}$ diminished pseudo-uridinilation of RNA. ${ }^{45}$ Usually this is indicative of a destabilization of cellular processes, ${ }^{46}$ suggesting that CIP48 and WCFS1 consumption is not beneficial for a mildly stressed intestinal environment.

Full genome sequencing of L. plantarum CIP104448 and L. plantarum TIFN101 was applied to identify possible gene clusters that might be responsible for the differential biological effects of the three L. plantarum strains. Several hundred novel L. plantarum genes were found in strain CIP48 (340 new OGs) and TIFN101 (177 OGs) as compared to strain WCFS1. Only a small number of these (47 OGs) are shared by both CIP48 and TIFN101. The majority of these novel genes appear to be located on plasmids. CIP48 appears to have several plasmids that are not present in TIFN101 or WCFS1 (see Table 4.5, supplementary data). Strain CIP48 has specific genes, such as for lantibiotic biosynthesis and several cell-surface proteins, that might explain its differential effect. Notably, however, there are also numerous genes/gene clusters in L. plantarum CIP104448 and L. plantarum TIFN101 that are not present in the WCFS1 genome. Strikingly, L. plantarum CIP104448 completely and L. plantarum TIFN101 partially lack the plantaricin biosynthesis clusters. These genes have, in previous studies, been linked to strain differences in cytokine production ${ }^{6,47}$ but were shown here not to be associated with immune effects in vivo. Also, L. plantarum CIP104448 and L. plantarum TIFN101 lack very large regions important for sugar metabolism. These differences have been attributed to adaptations to environmental factors which, in our opinion, are interesting targets genes and possibly associated with probiotic effects. ${ }^{48}$ Not only the presence but also the absence of genes may enhance immune effects of bacteria. ${ }^{4}$

This comparative genomics study, in which effects of $L$. plantarum supplementation on the mucosal transcriptome were combined with systemic immune activation parameters, provides many leads for follow-up experimental work to identify genes that are responsible for or involved in the observed differences in immune effects in humans. 


\section{References}

1 Kim HJ, Kim YJ, Lee SH, Yu J, Jeong SK, Hong SJ. Effects of Lactobacillus rhamnosus on allergic march model by suppressing Th2, Th17, and TSLP responses via CD4(+)CD25(+)Foxp3(+) Tregs. Clin Immunol 2014;153:178-86.

2 Smelt MJ, de Haan BJ, Bron PA, van Swam I, Meijerink M, Wells JM, et al. L. plantarum, L. salivarius, and L. lactis attenuate Th2 responses and increase Treg frequencies in healthy mice in a strain dependent manner. PLoS One 2012; 7:e47244.

3 Smelt MJ, de Haan BJ, Bron PA, van Swam I, Meijerink M, Wells JM, et al. Probiotics can generate FoxP3 T-cell responses in the small intestine and simultaneously inducing CD4 and CD8 T cell activation in the large intestine. PLoS One 2013;8:e68952.

4 Smelt MJ, de Haan BJ, Bron PA, van Swam I, Meijerink M, Wells JM, et al. The impact of Lactobacillus plantarum WCFS1 teichoic acid D-alanylation on the generation of effector and regulatory T-cells in healthy mice. PLoS One 2013;8:e63099.

5 Steinberg RS, Lima M, Gomes de Oliveira NL, Miyoshi A, Nicoli JR, Neumann E, et al. Effect of intestinal colonisation by two Lactobacillus strains on the immune response of gnotobiotic mice. Beneficial microbes 2014:1-11.

6 Meijerink M, van HS, Taverne N, Wels M, de VP, Bron PA, et al. Identification of genetic loci in Lactobacillus plantarum that modulate the immune response of dendritic cells using comparative genome hybridization. PLoSOne 2010;5:e10632.

7 van Hemert S, Meijerink M, Molenaar D, Bron PA, de Vos P, Kleerebezem M, et al. Identification of Lactobacillus plantarum genes modulating the cytokine response of human peripheral blood mononuclear cells. BMCMicrobiol 2010;10:293.

8 Akatsu H, Arakawa K, Yamamoto T, Kanematsu T, Matsukawa N, Ohara H, et al. Lactobacillus in jelly enhances the effect of influenza vaccination in elderly individuals. Journal of the American Geriatrics Society 2013;61:1828-30.

9 Bosch M, Mendez M, Perez M, Farran A, Fuentes MC, Cune J. Lactobacillus plantarum CECT7315 and CECT7316 stimulate immunoglobulin production after influenza vaccination in elderly. Nutricion hospitalaria 2012;27:504-9.

10 de Azevedo MS, Innocentin S, Dorella FA, Rocha CS, Mariat D, Pontes DS, et al. Immunotherapy of allergic diseases using probiotics or recombinant probiotics. Journal of applied microbiology 2013;115:319-33.

11 Licciardi PV, Tang ML. Vaccine adjuvant properties of probiotic bacteria. Discovery medicine 2011;12:525-33.

12 Van Puyenbroeck K, Hens N, Coenen S, Michiels B, Beunckens C, Molenberghs G, et al. Efficacy of daily intake of Lactobacillus casei Shirota on respiratory symptoms and influenza vaccination immune response: a randomized, double-blind, placebo-controlled trial in healthy elderly nursing home residents. The American journal of clinical nutrition 2012;95:1165-71.

13 West NP, Cripps AW. Are vaccination models suitable to determine whether probiotics have beneficial health effects in the general population? Human vaccines \& immunotherapeutics 2013;9:621-4.

14 Yaqoob P. Ageing, immunity and influenza: a role for probiotics? The Proceedings of the Nutrition Society 2014;73:309-17.

15 van Baarlen P, Troost F, van der Meer C, Hooiveld G, Boekschoten M, Brummer RJ, et al. Human mucosal in vivo transcriptome responses to three lactobacilli indicate how probiotics may modulate human cellular pathways. Proc Natl Acad Sci U S A 2011;108:4562-9. doi: 10.1073/pnas.1000079107. Epub 2010 Sep 7.

16 van Baarlen P, Troost FJ, van Hemert S, van der Meer C, de Vos WM, de Groot PJ, et al. Differential NFkappaB pathways induction by Lactobacillus plantarum in the duodenum of healthy humans correlating with immune tolerance. Proc Natl Acad Sci U S A 2009;106:2371-6. doi: 10.1073/pnas.0809919106. Epub 2009 Feb 3.

17 van Baarlen P, Troost FJ, van Hemert S, van der Meer C, de Vos WM, de Groot PJ, et al. Differential NFkappaB pathways induction by Lactobacillus plantarum in the duodenum of healthy humans correlating with immune tolerance. Proc Natl Acad Sci U S A 2009;106:2371-6. 
18 Lee OY, Kang DH, Lee DH, Chung IK, Jang JY, Kim Jl, et al. A comparative study of DA-9601 and misoprostol for prevention of NSAID-associated gastroduodenal injury in patients undergoing chronic NSAID treatment. Archives of pharmacal research 2014.

19 Mokhtare M, Valizadeh SM, Emadian O. Lower Gastrointestinal Bleeding due to Non-Steroid AntiInflammatory Drug-Induced Colopathy Case Report and Literature Review. Middle East journal of digestive diseases 2013;5:107-11.

20 Tomita T, Sadakata H, Tamura M, Matsui H. Indomethacin-induced generation of reactive oxygen species leads to epithelial cell injury before the formation of intestinal lesions in mice. Journal of physiology and pharmacology : an official journal of the Polish Physiological Society 2014;65:435-40.

21 Fornai M, Antonioli L, Colucci R, Pellegrini C, Giustarini G, Testai L, et al. NSAID-induced enteropathy: are the currently available selective COX-2 inhibitors all the same? The Journal of pharmacology and experimental therapeutics 2014;348:86-95.

22 Tanaka A, Hase S, Miyazawa T, Ohno R, Takeuchi K. Role of cyclooxygenase (COX)-1 and COX-2 inhibition in nonsteroidal anti-inflammatory drug-induced intestinal damage in rats: relation to various pathogenic events. The Journal of pharmacology and experimental therapeutics 2002;303:1248-54.

23 Higuchi K, Umegaki E, Watanabe T, Yoda Y, Morita E, Murano M, et al. Present status and strategy of NSAIDs-induced small bowel injury. Journal of gastroenterology 2009;44:879-88.

24 Kawada M, Arihiro A, Mizoguchi E. Insights from advances in research of chemically induced experimental models of human inflammatory bowel disease. World J Gastroenterol 2007;13:5581-93.

25 Smalley WE, Ray WA, Daugherty JR, Griffin MR. Nonsteroidal anti-inflammatory drugs and the incidence of hospitalizations for peptic ulcer disease in elderly persons. American journal of epidemiology 1995;141:539-45.

26 Kleerebezem M, Boekhorst J, van Kranenburg R, Molenaar D, Kuipers OP, Leer R, et al. Complete genome sequence of Lactobacillus plantarum WCFS1. Proc Natl Acad Sci U S A 2003;100:1990-5.

27 Bjarnason I, Hayllar J, MacPherson AJ, Russell AS. Side effects of nonsteroidal anti-inflammatory drugs on the small and large intestine in humans. Gastroenterology 1993;104:1832-47.

28 Troost FJ, Saris WH, Brummer RJ. Recombinant human lactoferrin ingestion attenuates indomethacininduced enteropathy in vivo in healthy volunteers. Eur J Clin Nutr 2003;57:1579-85.

29 Lin K, Kools H, de Groot PJ, Gavai AK, Basnet RK, Cheng F, et al. MADMAX - Management and analysis database for multiple omics experiments. Journal of integrative bioinformatics 2011;8:160.

30 Dai M, Wang P, Boyd AD, Kostov G, Athey B, Jones EG, et al. Evolving gene/transcript definitions significantly alter the interpretation of GeneChip data. Nucleic acids research 2005;33:e175.

31 Irizarry RA, Hobbs B, Collin F, Beazer-Barclay YD, Antonellis KJ, Scherf U, et al. Exploration, normalization, and summaries of high density oligonucleotide array probe level data. Biostatistics (Oxford, England) 2003;4:249-64.

32 Smyth GK. Linear models and empirical bayes methods for assessing differential expression in microarray experiments. Statistical applications in genetics and molecular biology 2004;3:Article3.

33 Sartor MA, Tomlinson CR, Wesselkamper SC, Sivaganesan S, Leikauf GD, Medvedovic M. Intensity-based hierarchical Bayes method improves testing for differentially expressed genes in microarray experiments. BMC bioinformatics 2006;7:538.

34 Siezen RJ, Francke C, Renckens B, Boekhorst J, Wels M, Kleerebezem M, et al. Complete resequencing and reannotation of the Lactobacillus plantarum WCFS1 genome. J Bacteriol 2012;194:195-6.

35 Jacobs R, Hintzen G, Kemper A, Beul K, Kempf S, Behrens G, et al. CD56bright cells differ in their KIR repertoire and cytotoxic features from CD56dim NK cells. Eur J Immunol 2001;31:3121-7.

36 Tarazona R, Casado JG, Delarosa O, Torre-Cisneros J, Villanueva JL, Sanchez B, et al. Selective depletion of CD56(dim) NK cell subsets and maintenance of CD56(bright) NK cells in treatment-naive HIV-1seropositive individuals. J Clin Immunol 2002;22:176-83.

37 Huang J, Jochems C, Anderson AM, Talaie T, Jales A, Madan RA, et al. Soluble CD27-pool in humans may contribute to T cell activation and tumor immunity. J Immunol 2013;190:6250-8.

38 Raab M, Wang H, Lu Y, Smith X, Wu Z, Strebhardt K, et al. T cell receptor "inside-out" pathway via signaling module SKAP1-RapL regulates $\mathrm{T}$ cell motility and interactions in lymph nodes. Immunity 2010;32:541-56.

39 Zhang Y, Wang H. Integrin signalling and function in immune cells. Immunology 2012;135:268-75. 
40 Herren B, Burrows PD. B cell-restricted human mb-1 gene: expression, function, and lineage infidelity. Immunologic research 2002;26:35-43.

41 Bratkovic T, Rogelj B. Biology and applications of small nucleolar RNAs. Cellular and molecular life sciences : CMLS 2011;68:3843-51.

42 Esteller M. Non-coding RNAs in human disease. Nature reviews Genetics 2011;12:861-74.

43 Williams GT, Farzaneh F. Are snoRNAs and snoRNA host genes new players in cancer? Nature reviews Cancer 2012;12:84-8.

44 Kiss-Laszlo Z, Henry Y, Bachellerie JP, Caizergues-Ferrer M, Kiss T. Site-specific ribose methylation of preribosomal RNA: a novel function for small nucleolar RNAs. Cell 1996;85:1077-88.

45 Kiss AM, Jady BE, Bertrand E, Kiss T. Human box H/ACA pseudouridylation guide RNA machinery. Molecular and cellular biology 2004;24:5797-807.

46 Su H, Xu T, Ganapathy S, Shadfan M, Long M, Huang TH, et al. Elevated snoRNA biogenesis is essential in breast cancer. Oncogene 2014;33:1348-58.

47 Wells JM, Rossi O, Meijerink M, van BP. Epithelial crosstalk at the microbiota-mucosal interface. ProcNat|AcadSciUSA 2011;108 Suppl 1:4607-14.

48 Molenaar D, Bringel F, Schuren FH, de Vos WM, Siezen RJ, Kleerebezem M. Exploring Lactobacillus plantarum genome diversity by using microarrays. J Bacteriol 2005;187:6119-27. 


\section{Chapter 5}

\section{Markers for visceral hypersensitivity in IBS}

Markers for visceral hypersensitivity in patients with irritable bowel syndrome

Samefko Ludidi*, Zlatan Mujagic*, Daisy MAE Jonkers, Daniel Keszthelyi, Martine AM Hesselink, Joanna W Kruimel, Jose M Conchillo, Ad AM Masclee

* Both authors contributed equally to the manuscript

Neurogastroenterology and Motility 2014;26:1104-11 


\begin{abstract}
Background

Irritable bowel syndrome (IBS) is a heterogeneous disorder with visceral hypersensitivity as important hallmark. It is not known whether IBS patients with visceral hypersensitivity have different epidemiological and clinical characteristics compared to IBS patients without visceral hypersensitivity. Aim of our study was to compare in detail a large group of hyper- versus normosensitive IBS patients with respect to epidemiological and clinical characteristics.
\end{abstract}

\title{
Methods
}

IBS patients (Rome III criteria) have been recruited for a large-scale cohort study. All patients form this cohort that underwent a rectal barostat procedure were included and allocated based on those with and without visceral hypersensitivity. Patient demographics, and symptoms were collected using questionnaires (GSRS, HADS, SF-36) and a 14-day symptom diary for IBS-related symptoms. A multivariate logistic regression model was used to identify risk markers for having visceral hypersensitivity.

\section{Key results}

Ninety-five normosensitive and 93 hypersensitive IBS patients participated in this study. Hypersensitive patients had significantly higher scores for GSRS abdominal pain $(p<0.05)$, indigestion, reflux and constipation syndrome (all $p<0.01$ ) and IBS-symptom intensity, discomfort, (both $p<0.05$ ) and mean symptom composite score $(p<0.01)$. Age, female sex and the use of SSRI medication were significantly different between the normo- and the hypersensitive IBS patients. However, after adjustment for other risk markers, only increasing age was found to be significantly associated with lower odds for having hypersensitivity (OR 0.97 (95\% Cl: 0.94; 0.99)).

\section{Conclusion}

Apart from more severe symptomatology, hypersensitive IBS patients are characterized by significantly younger age compared to normosensitve IBS patients. 


\section{Introduction}

Irritable bowel syndrome (IBS) is a functional disorder of the gastrointestinal (GI) tract and is characterized by altered bowel habits, associated with abdominal pain. The diagnosis is based on Rome III criteria, and includes four predominant subtypes i.e. IBS with diarrhoea (IBS-D), constipation (IBS-C), mixed (IBS-M) and the unspecified subtype (IBS-U).

Patients with IBS have a heterogeneous phenotypical appearance. A multifactorial pathophysiology is assumed to be key in this diversity. It has been hypothesized that increased visceral perception or visceral hypersensitivity is a major pathophysiological characteristic involved in the aetiology of IBS patients. A substantial subset of up to $60 \%$ of patients with IBS is characterized by visceral hypersensitivity, which is therefore considered an important hallmark of IBS. ${ }^{1,2}$

Using the rectal barostat, mechanical balloon distensions are applied to the rectum, allowing to discriminate between patients with and without visceral hypersensitivity ${ }^{1}$. Visceral hypersensitivity comprises both hyperalgesia (increased responses to painful stimuli) and allodynia (painful responses to non-noxious stimuli). In addition, it has been suggested that IBS patients with visceral hypersensitivity also exhibit increased responses to somatic stimuli, pointing to a more generalized (somatic and visceral) hypersensitivity in these patients. ${ }^{3,4}$ These findings however, have not been confirmed by others. ${ }^{5}$ Since visceral hypersensitivity is frequent in IBS and increased visceroperception in IBS patients is associated with higher severity of self-reported symptoms, ${ }^{6,7}$ patients with visceral hypersensitivity may represent a separate entity within the IBS population, not only from a pathophysiological but also from a clinical perspective. However, a more detailed characterization of potential differences in clinical features between patients with and without visceral hypersensitivity is currently lacking.

The aim of the present study was therefore to compare demographical, psychological, lifestyle, as well as medication-related risk markers for having visceral hypersensitivity in patients with IBS. We hypothesize that the female sex and severity of psychological and gastro-intestinal symptoms are significantly associated with having visceral hypersensitivity. 


\section{Materials and methods}

The present study is part of a large prospective cohort study on the phenotypical and genotypical characterization of patients with IBS, of which 126 patients had participated in a previous study by our group ${ }^{2}$.

Subjects with IBS included in this study were seen at our outpatient department from the Maastricht University Medical Center+ between September 2009 and June 2013. All secondary and tertiary referral IBS patients were also seen at the motility research unit at the Maastricht University Medical Center+.

Prior to participation, subjects had signed an informed consent and the study protocol had been approved by the Maastricht University Medical Centre+ Committee of Ethics and was executed according to the Declaration of Helsinki $\left(59^{\text {th }}\right.$ general assembly of the WMA, Seoul, South Korea, Oct. 2008). The study has been registered in the US National Library of Medicine (http://www.clinicaltrials.gov, NCT00775060).

\section{Patients}

Between September 2009 and June 2013, IBS patients visiting the gastroenterology outpatient clinic of Maastricht University Medical Centre, were asked to participate. Patients with an organic disease cause were excluded. All subjects that were included complied with Rome III criteria for IBS. The present study only included patients from our IBS cohort who were willing to undergo a rectal barostat procedure for the assessment of visceral perception. Patients could not participate if they were unable to stop medication that can influence gastrointestinal motility or perception for at least 3 days prior to the test.

Furthermore, all patients that participated in the IBS cohort had filled out questionnaires on demographics, symptoms, medical and lifestyle factors. In addition, they kept a symptom diary for 14 days.

\section{Rectal barostat}

Participants arrived in the hospital after an overnight fast. Rectal perception was measured using an electronic barostat (Distender II; G\&J Electronics, Toronto, ON, Canada, part: C7-CB-R) and a balloon of non-compliant material (Mui Scientific, Missisauga, ON, Canada, part: C7-2CB-R) was lubricated with KY-gel (Johnsson \& Joshnsson, Longhorne, PA, USA). The procedure was executed according to our standard protocol as previously described ${ }^{9}$. Accordingly, pain was scored, using a visual analogue scale (VAS) ranging between 0-100 mm. For subjects that did not complete the full protocol, VAS-scores for the remaining pressure steps were replenished, using the highest VAS-score achieved over the protocol thus far. Cut-off for visceral hypersensitivity was defined a VAS score of $\geq 20 \mathrm{~mm}$ at pressure step $26 \mathrm{mmHg}$. 
Furthermore, the percentage of subjects with allodynia, i.e. early pain perception in response to non-noxious stimuli, was calculated based on VAS $>10 \mathrm{~mm}$ prior to the pressure step indicative for visceral hypersensitivity (i.e. $26 \mathrm{mmHg}$ ) as previously described by our group. ${ }^{2}$

\section{Questionnaires}

Within a period of 2 weeks prior to the rectal barostat, questionnaires on e.g. demographics, lifestyle factors (smoking and drinking behaviour), stress events in relation to the presence of symptoms, symptom onset and the use of medication were completed. Furthermore, the presence of GI symptoms was assessed using the validated gastrointestinal symptom rating scale (GSRS), consisting of 16 items clustered into 5 major Gl syndromes: abdominal pain (AP), reflux syndrome (RS) diarrhoea syndrome (DS), indigestion syndrome (IS), constipation syndrome (CS). ${ }^{10}$ Anxiety and depression were assessed using the hospital anxiety and depression scale (HADS). A score $\geq 8$ on either the anxiety or depression scale were indicative for the presence of anxiety or depressive symptoms, respectively. ${ }^{11,12}$ The RAND-36 questionnaire, which is the validated Dutch version of the Medical Outcomes Study 36-item Short Form Health Survey (SF-36), ${ }^{13}$ was used to assess generic health-related quality of life (QoL). The SF36 consists of 8 domains, all ranging between $0-100$, of which a mental (QoL-MCS) and physical (QoL-PCS) composite score can be derived. ${ }^{14,15}$ Finally, IBS-related symptoms of discomfort, abdominal pain, bloating, flatulence, nausea as well as perceived intensity of symptoms were scored on a 5-point Likert-scale between 1-5 for 14 consecutive days prior to the rectal barostat procedure. Subsequently, the mean, i.e. per day, symptom composite score (MSS) was calculated (range: 1-5), based on sum of individual symptoms.

\section{Data and statistical analysis}

Based on barostat data, patients were defined as normosensitive (IBS NORM $_{\text {) or }}$ hypersensitive (IBS ${ }_{\mathrm{HYP}}$ ) to rectal distension. Data were analysed for normality using a normal probability plot. Subsequently, descriptives are presented as mean or percentages as appropriate, for the whole group of IBS patients (IBS TOTAL) as well as for the two IBS subgroups. Dichotomous data were analysed using a Chi-square test, continuous and ordinal data were analysed using an independent t-test.

Next, a multivariate logistic regression model was used to identify risk markers for having visceral hypersensitivity. The following variables were included in the model: I. demographical characteristics (i.e. age, sex, BMI, IBS-subtype and symptom duration) II. psychological symptoms (i.e. anxiety and depressive symptoms) III. lifestyle factors (i.e. smoking history and use of alcohol) and IV. the use of medication (i.e. NSAID and SSRI). Adjusted data were shown as odds ratios (OR) and 95\% confidence intervals (CI). 
Finally, a Pearson's correlation test was performed to correlate visceroperception with symptoms and age.

Statistical analyses were performed using IBM SPSS Statistics version 20.0 (IBM Statistics for Macintosh, Chicago IL) and a two-sided $p<0.05$ was considered statistically significant.

\section{Results}

\section{Demographical characteristics and visceroperception}

A total of 188 IBS patients underwent a rectal barostat procedure, of which 93 (49.5\%) patients were found to be hypersensitive. In Figure 5.1 VAS-scores for pain perception

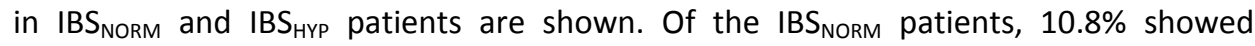
allodynia compared to. $89.2 \%$ in the IBS $S_{\text {HYP }}$ patients $(p<0.001)$.

A detailed description of the patients' demographical characteristics, psychological symptoms, lifestyle factors and medication use is given in Table 5.1. Compared to $\mathrm{IBS}_{\text {NORM}}, \mathrm{IBS}_{\mathrm{HYP}}$ patients were significantly younger (36.9 \pm 1.62 vs. $45.8 \pm 1.71$ years; $p<0.001)$ and more frequently female $(81.7 \%$ vs. $66.0 \% ; p<0.05)$. No significant differences were observed in BMI, IBS subtype or symptom duration.

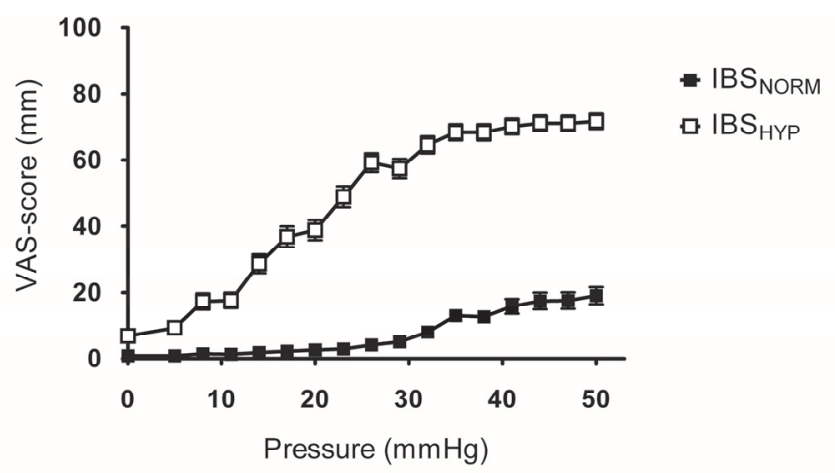

Figure 5.1 Visceroperception barostat data of normosensitive (IBS ${ }_{\text {NORMM }}$ ) and hypersensitive (IBS HYP $_{\text {) IBS }}$ patients for pain, expressed as visual analogue scale (VAS) scores versus intra-balloon pressure in $\mathrm{mmHg}$. 
Table 5.1 Patient characteristics based on demographical characteristics, psychological symptoms, lifestyle factors and the use of medication in all patients $(n=188)$ that underwent a barostat procedure and split for patients without $(n=95)$ and with $(n=93)$ visceral hypersensitivity.

\begin{tabular}{|c|c|c|c|}
\hline Parameter & $\mathrm{IBS}_{\text {TOTAL }}$ & $\mathrm{IBS}_{\text {NORM }}$ & $\mathrm{IBS}_{\mathrm{HYP}}$ \\
\hline \multicolumn{4}{|l|}{ I. Demographical characteristics } \\
\hline Age (years; mean \pm SEM) & $41.4 \pm 0.03$ & $45.8 \pm 1.71$ & $36.9 \pm 1.62 * * *$ \\
\hline Female sex (\%) & 73.9 & 66.0 & $81.7^{*}$ \\
\hline BMI & $24.4 \pm 0.33$ & $25.4 \pm 0.47$ & $23.4 \pm 0.48$ \\
\hline \multicolumn{4}{|l|}{ IBS subtype (\%) } \\
\hline IBS-D & 35.1 & 40.4 & 30.1 \\
\hline IBS-C & 20.2 & 17.0 & 23.7 \\
\hline IBS-M & 37.8 & 35.1 & 40.9 \\
\hline IBS-U & 6.9 & 7.4 & 5.4 \\
\hline Duration of symptoms (years) & $12.1 \pm 1.13$ & $11.7 \pm 1.60$ & $12.5 \pm 1.32$ \\
\hline Post-infectious IBS (\%) & 21.3 & 21.5 & 21.0 \\
\hline \multicolumn{4}{|c|}{ II. Psychological symptoms - anxiety and depressive symptoms (HADS) } \\
\hline Depressive symptoms, i.e. score of $\geq 8$ (\%) & 20.7 & 17.9 & 23.7 \\
\hline Anxiety symptoms, i.e. score of $\geq 8(\%)$ & 39.4 & 40.0 & 38.7 \\
\hline \multicolumn{4}{|l|}{ III. Lifestyle parameters } \\
\hline Positive smoking history (\%) & 48.4 & 47.4 & 49.5 \\
\hline Alcohol intake >15/week (\%) & 6.9 & 8.4 & 5.4 \\
\hline \multicolumn{4}{|c|}{ IV. Use of medication 2 weeks prior to participation } \\
\hline PPI (\%) & 22.9 & 25.3 & 20.4 \\
\hline NSAID (\%) & 12.2 & 14.7 & 9.6 \\
\hline SSRI (\%) & 11.2 & 6.3 & $16.1^{*}$ \\
\hline Motility +drugs (\%) & 18.1 & 15.8 & 20.4 \\
\hline Motility -drugs (\%) & 8.5 & 7.4 & 9.7 \\
\hline
\end{tabular}

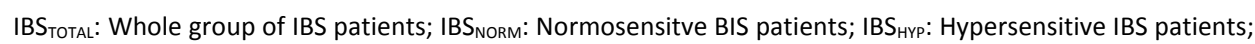
$\mathrm{PI}$ : post-infectious. ${ }^{*} \mathrm{p}<0.05$ vs. $\mathrm{IBS}_{\mathrm{NORM}} ;{ }^{* *} \mathrm{p}<0.001$ vs. IBS $_{\mathrm{NORM}}$.

When analyzing pain perception in more detail, we found that $52.1 \%(n=98)$ of the $\mathrm{IBS}_{\text {TOTAL }}$ patients were allodynic, i.e. experienced pain at low pressure ( $<26 \mathrm{mmHg}$ ). Similar to hypersensitive patients, patients with allodynia were significantly younger versus the non-allodynic patients $(36.5 \pm 1.64$ vs. $46.0 \pm 1.79 ; p<0.001)$ as well as more often of the female sex $(78.5 \%$ vs. $68.9 \%$; $p<0.05)$. No other differences were observed between patients with and without allodynia.

\section{Risk markers for having visceral hypersensitivity}

In Table 5.2 risk markers for visceral hypersensitivity are presented. After adjustment for other potential risk markers, only increasing age was found to be significantly associated with lower odds for having hypersensitivity (OR 0.97 (95\% Cl: 0.94; 0.99)). Also, age was found to correlate significantly though weakly with intensity of pain (VAS) at pressure step $26 \mathrm{mmHg}$ and age $(r=-0.253 ; p<0.01)$. In addition, medication use showed higher but not statistically significant odds for having visceral hypersensitivity in patients using SSRIs (OR: 2.6 (95\% Cl: 0.90; 7.52)). 
Table 5.2 Odds ratios for having visceral hypersensitivity in comparison to normosensitvity based on demographical characteristics, psychological symptoms, lifestyle factors and the use of medication.

\begin{tabular}{|c|c|c|c|}
\hline Parameter & Odds ratio & $95 \% \mathrm{Cl}$ & $\mathrm{p}$-value \\
\hline \multicolumn{4}{|l|}{ I. Demographical characteristics } \\
\hline Age (years) & 0.97 & $0.94 ; 0.99$ & 0.004 \\
\hline Sex (female reference) & 0.56 & $0.26 ; 1.20$ & 0.135 \\
\hline $\mathrm{BMI}\left(\mathrm{kg} / \mathrm{m}^{2}\right)$ & 0.94 & $0.87 ; 1.02$ & 0.134 \\
\hline Symptom duration (years) & 1.02 & $0.97 ; 1.05$ & 0.289 \\
\hline IBS subtype & 1.08 & $0.78 ; 1.50$ & 0.629 \\
\hline \multicolumn{4}{|l|}{ II. Psychological symptoms } \\
\hline Depressive symptoms & 1.32 & $0.54 ; 3.18$ & 0.543 \\
\hline Anxiety symptoms & 0.89 & $0.42 ; 1.90$ & 0.766 \\
\hline \multicolumn{4}{|l|}{ III. Lifestyle factors } \\
\hline Positive smoking history & 1.30 & $0.66 ; 2.55$ & 0.452 \\
\hline Use of alcohol >15 units/ week & 0.66 & $0.17 ; 2.52$ & 0.541 \\
\hline \multicolumn{4}{|l|}{ IV. Use of medication } \\
\hline Use of NSAID & 0.68 & $0.24 ; 1.90$ & 0.459 \\
\hline Use of SSRI & 2.60 & $0.90 ; 7.52$ & 0.077 \\
\hline
\end{tabular}

IBS $_{\text {NORM: }}$ : Normosensitve IBS patients; IBS ${ }_{\text {HYP: }}$ Hypersensitive IBS patients; $95 \% \mathrm{Cl}$ : 95\% confidence interval.

\section{Symptom scores and quality of life}

Scores for symptoms according to the GSRS and the IBS symptom diary, as well as QoL scores are given in Table 5.3. GSRS symptom scores for abdominal pain, reflux syndrome, indigestion syndrome and constipation syndrome were significantly increased in the IBS $\mathrm{HYP}_{\mathrm{H}}$ when compared to the IBS $\mathrm{INRM}_{\mathrm{NO}}$ group. Resulting from the

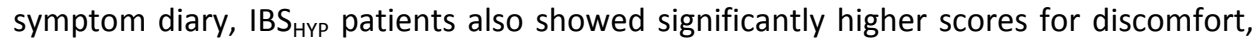
abdominal pain and intensity of symptoms. As a result, MSS was significantly increased

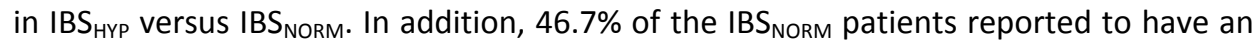
exacerbation of symptoms with stress versus $53.3 \%$ of the IBS ${ }_{\text {HYP }}$ patients $(p=0.41)$.

Correlating GSRS symptom scores with visceroperception (i.e. VAS-score at pressure step $26 \mathrm{mmHg}$ ), significantly positive, though weak correlations (all $r<0.25$ ) were observed for reflux syndrome, diarrhoea syndrome, indigestion syndrome and constipation syndrome $(p<0.05)$. No correlation was observed between GSRS for abdominal pain. Diary symptoms scores for discomfort $(p<0.01)$ abdominal pain $(p<0.01)$, intensity of symptoms $(p<0.01)$ and MSS $(p<0.05)$ showed significant correlations with visceroperception scores (all $r<0.28$; data not shown).

Regarding quality of life, no differences were observed between IBS NORM $_{\text {and IBS }}$ aYP with respect to QoL-PCS and QoL-MCS (Table 5.3). 
Table 5.3 Symptoms and quality of life in patients without $(n=95)$ and with $(n=93)$ visceral hypersensitivity (IBS $_{\text {NORM }}$ and IBS ${ }_{\text {HYP }}$, respectively).

\begin{tabular}{lcc}
\hline Parameter & IBS $_{\mathrm{NORM}}$ & IBS $_{\mathrm{HYP}}$ \\
\hline GSRS syndrome (Mean \pm SEM) & & $3.57 \pm 0.13^{*}$ \\
Abdominal pain & $3.15 \pm 0.13$ & $2.29 \pm 0.13^{* *}$ \\
Reflux syndrome & $1.73 \pm 0.13$ & $3.54 \pm 0.17$ \\
Diarrhoea syndrome & $3.26 \pm 0.18$ & $4.24 \pm 0.15^{* *}$ \\
Indigestion syndrome & $3.69 \pm 0.13$ & $3.62 \pm 0.17^{* *}$ \\
Constipation syndrome & $2.99 \pm 0.14$ & \\
SF-36 (Mean \pm SEM) & & $39.01 \pm 1.14$ \\
QoL-PCS & $40.90 \pm 1.18$ & $46.33 \pm 1.16$ \\
QoL-MSC & $48.48 \pm 1.20$ & \\
Symptom scores - 14 day average (Mean \pm SEM) & & $2.61 \pm 0.80^{*}$ \\
Discomfort & $2.31 \pm 0.09$ & $2.52 \pm 0.10^{* *}$ \\
Abdominal pain & $2.11 \pm 0.10$ & $2.32 \pm 0.11$ \\
Bloating & $2.02 \pm 0.12$ & $2.24 \pm 0.10$ \\
Flatulence & $2.21 \pm 0.11$ & $1.79 \pm 0.11$ \\
Nausea & $1.52 \pm 0.81$ & $2.72 \pm 0.09^{*}$ \\
Intensity of symptoms & $2.38 \pm 0.10$ & $14.2 \pm 0.40^{* *}$ \\
Mean symptom composite score & $12.49 \pm 0.48$ & \\
\hline
\end{tabular}

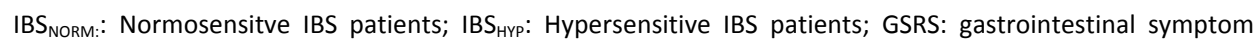
rating scale; SF-36: 36-item short form health survey; PCS: physical quality of life composite score; MCS: mental quality of life composite score. ${ }^{*} \mathrm{p}<0.05$ vs. $\mathrm{IBS}_{\mathrm{NORM}}{ }^{* *} \mathrm{p}<0.01 \mathrm{vs}$. IBS $\mathrm{INORM}^{* * *} \mathrm{p}<0.001$ vs. IBS $\mathrm{IORM}_{\text {. }}$.

\section{Effect of psychological status on symptoms}

GSRS symptoms abdominal pain and indigestion syndrome were found significantly affected by HADS-A scores ( $p=0.02$ and 0.03 , respectively), but not by HADS-D scores. IBS-diary symptom scores for discomfort $(\mathrm{p}=0.01)$, abdominal pain and symptom intensity and MSS (all $p=0.04$ ) on the other hand, were found to be significantly affected by the HADS-D scores, but not by HADS-A. Correcting for HADS-A and HADS-D however, did not alter the results as symptom scores for the abovementioned parameters remained significantly increased in the hyper- versus the normosensitive IBS patients

\section{Discussion}

We have demonstrated that visceral hypersensitivity in patients with IBS is associated with younger age, female sex and use of SSRI medication. Having visceral hypersensitivity was also associated with significantly increased GSRS scores and IBSrelated symptom scores, compared to patients without visceral hypersensitivity. In the multivariate analysis, only age remained a statistically relevant marker of visceral hypersensitivity. 
Hypersensitive patients were more frequently younger of age when compared to normosensitive IBS patients. Given the fact that symptom duration was not significantly different among hyper- and normosensitive patients, the patients with visceral hypersensitivity have presented at a younger age. Previous data point to a decline in visceral pain perception with aging, showing higher pain thresholds to rectal distension in older (i.e. $86 \pm 4$ years) compared to younger (i.e. $26 \pm 1$ years) healthy subjects. ${ }^{16}$ Pain perception to lower range pressure distensions points to an allodynic type response. Most patient in the hypersensitive group also had allodynia. It has been observed that ageing results in a decrease in the number of sensory neurons in the intestine. ${ }^{17,18}$ This may account, at least in part, for the difference in age between the hyper- and normosensitive group. Anticipatory responses on the other hand, may also contribute. Pain sensitivity in general, but also visceral hypersensitivity has been associated with an increased tendency to report symptoms, ${ }^{19}$ pointing to involvement of central mechanisms, e.g. altered coping and potential and psychological factors such as anxiety in symptom presentation in IBS. $^{20,21}$ In line with these observations, we recently demonstrated that dysfunctional cognitions, which are related to coping, independently affect IBS symptomatology. ${ }^{22}$ Accordingly, a decreased perceptual response to stimuli may occur over time. As previously described this is most likely due to adapting coping strategies and habituation. ${ }^{20,23}$ Although desensitization over time could also have accounted for the significant age discrepancy in our study population (i.e. normo- versus hypersensitives), symptom duration was not different between the two groups. Therefore, we assume that hypersensitive patients have a different underlying pathophysiology, not necessarily only pain-associated anticipation as proposed by Dorn et al. with altered coping mechanisms over time ${ }^{19}$.

The hypersensitive IBS patients were not only significantly younger but also more frequently female. Concerning gender differences, some groups have observed higher responses to painful stimuli (i.e. lower threshold or tolerance) in women compared to men, ${ }^{24}$ which may account for the female predominance in the hypersensitive group. The influence of sex hormones may give rise to such differences between women and men. Accounting for menstrual cycle when analysing visceral sensitivity could have given better insight in the potential association between sex hormones and visceral sensitivity. Unfortunately, these data were not available in our population from daily clinical practice. After correction for potentially confounding factors in the multivariate model female sex was no longer found to be a risk marker for visceral hypersensitivity in our IBS population.

Alcohol intake and smoking are well-known lifestyle risk factors associated with several gastrointestinal and liver diseases and carcinogenesis. With regard to IBS, several studies have reported that lifestyle factors are associated with severity of symptoms. $^{25-28}$ 
Previously, we have observed that after acute alcohol intake intestinal permeability in healthy subjects increases. ${ }^{29}$ Increased intestinal permeability has been related to symptom severity and may contribute to visceral hypersensitivity. ${ }^{30}$ We evaluated whether alcohol and smoking behaviour were associated with visceral perception, but could not find significant associations between these risk markers and visceral pain perception.

A subset of IBS patients develops symptoms following acute gastroenteritis. ${ }^{31}$ This specific form of IBS is referred to as post-infectious (PI) IBS. PI-IBS is typically characterized by low-grade inflammation, ${ }^{32}$ which may contribute to the development of pain symptoms. ${ }^{33}$ In the present study, we used a questionnaire as a screening tool for IBS including questions related to PI-IBS. No differences were observed in PI-IBS prevalence between hyper and normosensitive patients (20 vs. $21 \%$, respectively). Percentages we found are within the range of those observed in other studies and cover the range in which patients report IBS symptom onset, following acute gastroenteritis. $^{32,34}$

Anxiety and depression are more prevalent among IBS patients compared to control subjects. Several studies have shown that $20-30 \%$ of IBS patients have depressive symptoms and $15-45 \%$ anxiety symptoms. ${ }^{22,35}$ Associations have been found between anxiety and depression and the severity of reported symptoms in IBS. ${ }^{36-38}$ Previously, Dorn et al. found that IBS patients with higher scores on psychometric scales for anxiety and depression have an increased tendency to report symptoms during the barostat procedure. ${ }^{19}$ Although we compared a substantial number of IBS patients, psychological distress as measured with the HADS was equally distributed among patients with and without visceral hypersensitivity. Neither did absolute scores for HADS-A and HADS-D differ between the normosensitive versus the hypersensitive patients nor did intensity of pain correlate with HADS score (data not shown). Based on our data we assume that anxiety and depression are likely to be associated with having IBS per se, but not with having visceral hypersensitivity.

Concerning medication, use of SSRI was more frequent among the hypersensitive compared to the normosensitive patients. This was statistically significant in the univariate analysis $(16.1 \%$ vs. $6.3 \%$, respectively; $p<0.05)$ but not in the multivariate analysis $(p=0.077)$. SSRI medication is more frequently prescribed among the IBS population compared to the general population due to an increased prevalence of anxiety and depression in IBS. ${ }^{22,35}$ On the other hand, SSRI's are also prescribed for control of abdominal symptoms, especially pain. ${ }^{39}$ As symptoms are more pronounced in hypersensitive patients, as shown by GSRS and symptom diary scores, this may account for higher percentage of SSRI users in the hypersensitive group. 
Hypersensitive IBS patients represent a patient group with more pronounced symptoms during provocation (mechanical distension) with up to $90 \%$ also with early onset of pain, referred to as allodynia. Previous studies indicate that pain perception during provocation not necessarily correlates with symptom scores in daily life and Posserud et al. previously demonstrated that GSRS scores only moderately correlated with pain thresholds assessed by the rectal barostat ${ }^{7}$. Sabate et al. found no correlation between pain thresholds after rectal distensions and IBS symptom severity. ${ }^{40}$ In a previous study by our group, we demonstrated that IBS symptoms were increased in patients with visceral hypersensitivity but not in those without hypersensitivity. ${ }^{6}$ In the present study also symptoms according to the GSRS (i.e. abdominal pain, constipation syndrome, reflux syndrome and indigestion syndrome) were significantly increased in the hypersensitive IBS patients. Of these symptoms, constipation, reflux and indigestion syndrome weakly correlated with visceral sensitivity thresholds. Also with regards to the IBS symptom diary scores, we only found weak correlations (i.e. for discomfort, abdominal pain, intensity of symptoms and mean symptom score). In this regard we should realize that the barostat is a symptom provocation test that measures threshold and intensity of pain in response to stimulation. With scores as GSRS and with diaries, reporting pain retrospectively (e.g. at the end of the day when using a diary) may give rise to recall bias. Although abdominal pain and indigestion syndrome (derived from the GSRS) were significantly affected by the presence of anxiety symptoms (HADS) and discomfort, abdominal pain, intensity of symptoms and MSS, were significantly affected by the presence of depressive symptoms, Correction for psychological comorbidity (HADS-A and HADS-D) did not alter the symptom scores in hypersensitive IBS patients, nor the difference between hyper- and normosensitive patients. This observation supports the concept that not only visceroperception but also altered psychological status affects symptom generation in IBS, supporting a role of the brain-gut axis in these patients.

In conclusion, we have compared a substantial group of IBS patients, with and without visceral hypersensitivity in search of patient characteristics and markers for visceral hypersensitivity. Our data show that IBS patients with visceral hypersensitivity are significantly younger and report significantly increased intensity of gastrointestinal symptoms, but no other characteristics or markers could be discerned. 


\section{References}

1. Mertz H, Naliboff B, Munakata J, Niazi N, Mayer EA. Altered rectal perception is a biological marker of patients with irritable bowel syndrome. Gastroenterology. 1995;109: 40-52.

2. Ludidi S, Conchillo JM, Keszthelyi D, Van Avesaat M, Kruimel JW, Jonkers DM, Masclee AA. Rectal hypersensitivity as hallmark for irritable bowel syndrome: defining the optimal cutoff. Neurogastroenterol Motil. 2012;24:729-33.

3. Piche $M$, Arsenault $M$, Poitras $P$, Rainville $P$, Bouin $M$. Widespread hypersensitivity is related to altered pain inhibition processes in irritable bowel syndrome. Pain. 2010;148:49-58.

4. Zhou Q, Fillingim RB, Riley JL, 3rd, Malarkey WB, Verne GN. Central and peripheral hypersensitivity in the irritable bowel syndrome. Pain. 2010;148:454-61.

5. Whitehead WE, Holtkotter B, Enck P, Hoelzl R, Holmes KD, Anthony J, Shabsin HS, Schuster MM. Tolerance for rectosigmoid distention in irritable bowel syndrome. Gastroenterology. 1990;98:1187-92.

6. van der Veek PP, Van Rood YR, Masclee AA. Symptom severity but not psychopathology predicts visceral hypersensitivity in irritable bowel syndrome. Clin Gastroenterol Hepatol. 2008;6:321-8.

7. Posserud I, Syrous A, Lindstrom L, Tack J, Abrahamsson H, Simren M. Altered rectal perception in irritable bowel syndrome is associated with symptom severity. Gastroenterology. 2007;133:1113-23.

8. Zhou Q, Verne GN. New insights into visceral hypersensitivity--clinical implications in IBS. Nat Rev Gastroenterol Hepatol. 2011;8:349-55.

9. Ludidi S, Conchillo JM, Keszthelyi D, Koning CJ, Vanhoutvin SA, Lindsey PJ, Leufkens AM, Kruimel JW, Jonkers DM, Masclee AA. Does meal ingestion enhance sensitivity of visceroperception assessment in irritable bowel syndrome? Neurogastroenterol Motil. 2012;24:47-53, e3.

10. Svedlund J, Sjodin I, Dotevall G. GSRS--a clinical rating scale for gastrointestinal symptoms in patients with irritable bowel syndrome and peptic ulcer disease. Dig Dis Sci 1988;33:129-34.

11. Zigmond AS, Snaith RP. The hospital anxiety and depression scale. Acta Psychiatr Scand. 1983;67: 361-70.

12. Bjelland I, Dahl AA, Haug TT, Neckelmann D. The validity of the Hospital Anxiety and Depression Scale. An updated literature review. J Psychosom Res. 2002;52:69-77.

13. Zee vd. Het meten van de algemene gezondheidstoestand met de RAND-36, een handleiding [Assessment of general health status with the RAND-36; guidebook]. 1993.

14. Creed F, Ratcliffe J, Fernandez L, Tomenson B, Palmer S, Rigby C, Guthrie E, Read N, Thompson D. Health-related quality of life and health care costs in severe, refractory irritable bowel syndrome. Ann Intern Med. 2001;134:860-8.

15. Farivar SS, Cunningham WE, Hays RD. Correlated physical and mental health summary scores for the SF-36 and SF-12 Health Survey, V.I. Health Qual Life Outcomes 2007;5:54.

16. Lagier E, Delvaux M, Vellas B, Fioramonti J, Bueno L, Albarede JL, Frexinos J. Influence of age on rectal tone and sensitivity to distension in healthy subjects. Neurogastroenterol Motil. 1999;11:101-7.

17. Gabella G. Fall in the number of myenteric neurons in aging guinea pigs. Gastroenterology. 1989;96: 1487-93.

18. Meciano Filho J, Carvalho VC, de Souza RR. Nerve cell loss in the myenteric plexus of the human esophagus in relation to age: a preliminary investigation. Gerontology. 1995;41:18-21.

19. Dorn SD, Palsson OS, Thiwan SI, Kanazawa M, Clark WC, van Tilburg MA, Drossman DA, Scarlett Y, Levy RL, Ringel Y, Crowell MD, Olden KW, Whitehead WE. Increased colonic pain sensitivity in irritable bowel syndrome is the result of an increased tendency to report pain rather than increased neurosensory sensitivity. Gut. 2007;56:1202-9.

20. Aziz Q. Visceral hypersensitivity: fact or fiction. Gastroenterology. 2006;131:661-4.

21. Fichna J, Storr MA. Brain-Gut Interactions in IBS. Front Pharmacol. 2012;3:127.

22. Thijssen AY, Jonkers DM, Leue $C$, van der Veek PP, Vidakovic-Vukic $M$, van Rood YR, Clemens $C H$, Masclee AA. Dysfunctional cognitions, anxiety and depression in irritable bowel syndrome. J Clin Gastroenterol. 2010;44:e236-41.

23. Naliboff BD, Berman S, Suyenobu B, Labus JS, Chang L, Stains J, Mandelkern MA, Mayer EA. Longitudinal change in perceptual and brain activation response to visceral stimuli in irritable bowel syndrome patients. Gastroenterology 2006; 131:352-365. 
24. Unruh AM. Gender variations in clinical pain experience. Pain. 1996;65:123-67.

25. Dong L, Dingguo L, Xiaoxing X, Hanming L. An epidemiologic study of irritable bowel syndrome in adolescents and children in China: a school-based study. Pediatrics. 2005;116:e393-6.

26. Reding KW CK, Jarrett ME, Eugenio MD, Heitkemper MM. Relationship between patterns of alcohol consumption and gastrointestinal symptoms among patients with irritable bowel syndrome. Am J Gastroenterol. 2013;108:270-6.

27. Mearin F, Badía X, Balboa A, Baró E, Caldwell E, Cucala M, Díaz-Rubio M, Fueyo A, Ponce J, Roset M, Talley NJ. Irritable bowel syndrome prevalence varies enormously depending on the employed diagnostic criteria: comparison of Rome II versus previous criteria in a general population. Scand J Gastroenterol 2001;36:1155-61.

28. Li FX, Patten SB, Hilsden RJ, Sutherland LR. Irritable bowel syndrome and health-related quality of life: a population-based study in Calgary, Alberta. Can J Gastroenterol. 2003; 17: 259-263.

29. Elhaseen Elamin DJ, Freddy Troost, Jan Dekker, Ad Masclee. Tu1352 Moderate Dosage of Ethanol Increases Small and Large Intestinal Permeability in Healthy Volunteers. Gastroenterology. 2012; 142.

30. Zhou $Q$, Zhang $B$, Verne GN. Intestinal membrane permeability and hypersensitivity in the irritable bowel syndrome. Pain. 2009;146:41-6.

31. Ghoshal UC, Ranjan P. Post-infectious irritable bowel syndrome: the past, the present and the future. J Gastroenterol Hepatol. 2011;26 Suppl 3:94-101.

32. Spiller R, Lam C. An Update on Post-infectious Irritable Bowel Syndrome: Role of Genetics, Immune Activation, Serotonin and Altered Microbiome. J Neurogastroenterol Motil 2012;18:258-68.

33. Gecse K, Róka R, Ferrier L, Leveque M, Eutamene H, Cartier C, Ait-Belgnaoui A, Rosztóczy A, Izbéki F, Fioramonti J, Wittmann T, Bueno L. Increased faecal serine protease activity in diarrhoeic IBS patients: a colonic lumenal factor impairing colonic permeability and sensitivity. Gut. 2008;57:591-9.

34. Longstreth GF, Hawkey CJ, Mayer EA, Jones RH, Naesdal J, Wilson IK, Peacock RA, Wiklund IK. Characteristics of patients with irritable bowel syndrome recruited from three sources: implications for clinical trials. Aliment Pharmacol Ther 2001;15:959-64.

35. Lee S WJ, Ma YL, Tsang A, Guo WJ, Sung J. Irritable bowel syndrome is strongly associated with generalized anxiety disorder: a community study. Aliment Pharmacol Ther 2009;30:643-51.

36. Surdea-Blaga T, Baban A, Dumitrascu DL. Psychosocial determinants of irritable bowel syndrome. World journal of gastroenterology : WJG. 2012;18:616-26.

37. Labus JS, Bolus R, Chang L, Wiklund I, Naesdal J, Mayer EA, Naliboff BD. The Visceral Sensitivity Index: development and validation of a gastrointestinal symptom-specific anxiety scale. Aliment Pharmacol Ther. 2004;20:89-97.

38. Jerndal P, Ringström G, Agerforz P, Karpefors M, Akkermans LM, Bayati A, Simrén M. Gastrointestinalspecific anxiety: an important factor for severity of GI symptoms and quality of life in IBS. Neurogastroenterol Motil. 2010;22:646-e179.

39. Ruepert L, Quartero AO, de Wit NJ, van der Heijden GJ, Rubin G, Muris JW. Bulking agents, antispasmodics and antidepressants for the treatment of irritable bowel syndrome. The Cochrane database of systematic reviews 2011: CD003460.

40. Sabate JM VM, Mion F, Siproudhis L, Ducrotte P, Zerbib F, Grimaud JC, Dapoigny M, Dyard F, Coffin B. Relationship between rectal sensitivity, symptoms intensity and quality of life in patients with irritable bowel syndrome. Aliment Pharmacol Ther. 2008;28:484-90. 
Markers for visceral hypersensitivity in IBS 


\section{Chapter 6}

Biological markers for visceral hypersensitivity in IBS

Biomarkers for visceral hypersensitivity in patients with irritable bowel syndrome

Zlatan Mujagic, Daisy MAE Jonkers, Samefko Ludidi, Daniel Keszthelyi, Martine A Hesselink, Ruud N Kievit, Jessica F Althof, Zsa Zsa Weerts, Joanna W Kruimel, Frederik-Jan van Schooten, Ad AM Masclee

\section{Submitted}




\section{Abstract}

\section{Background}

Up to $60 \%$ of patients with Irritable Bowel Syndrome (IBS) have increased visceral perception. Intestinal inflammation, neuroendocrine activity and intraluminal metabolic processes may contribute to the development of visceral hypersensitivity. Previously, we found that markers that are indicative for these biological processes are altered in IBS patients compared to healthy controls. We hypothesize that normo- and hypersensitive IBS patients significantly differ in biomarker levels.

\section{Aim}

To get more insight in biological processes associated with altered visceroperception, several faecal and plasma markers were measured in normosensitive $\left(\right.$ IBS $_{\text {NORM }}$ ) and hypersensitive IBS patients (IBS $\left.{ }_{\mathrm{HYP}}\right)$.

\section{Methods}

IBS patients underwent a rectal barostat to assess visceral perception to pain and based on the outcome were classified as IBS NORM $_{\text {or IBS }}$ HYP. Calprotectin, human $\beta$-defensin 2 (HBD2), chromogranin A (CgA), and short chain fatty acids (SCFAs) were measured in faeces, citrulline in plasma and serotonin and its principal metabolite 5-hydroxyindoleacetic acid (5-HIAA) in platelet poor plasma (PPP).

\section{Key Results}

Faecal markers and plasma citrulline were measured in 83 IBS $_{\mathrm{HYP}}$ and $84 \mathrm{IBS}_{\mathrm{NORM}}$ patients, while PPP for the assessment of serotonin and 5-HIAA was available (for a subgroup, i.e.) $53 \mathrm{IBS}_{\mathrm{HYP}}$ and $42 \mathrm{IBS}_{\mathrm{NORM}}$. None of the tested markers showed significant differences between $\mathrm{IBS}_{\mathrm{HYP}}$ and $\mathrm{IBS}_{\mathrm{NORM}}$ patients. Results were not affected by confounders, such as patient characteristics or medication use.

\section{Conclusions}

Biomarkers relating to several pathophysiological mechanism did not differ between hypersensitive and normosensitive IBS patients. Our findings do not support a role for the relating biological processes in hypersensitive IBS patients based on these biomarkers. 


\section{Introduction}

Visceral hypersensitivity, measured by rectal balloon distention procedures, is an important hallmark of irritable bowel syndrome (IBS). The prevalence of visceral hypersensitivity among IBS patients ranges between $30 \%$ and $60 \% .^{1-5}$ In a previous study we found that hypersensitive IBS patients compared to normosensitive patients were younger, more often female, more often used selective serotonin reuptake inhibitors (SSRI's) and reported higher gastrointestinal (GI) symptom scores. ${ }^{4}$ The latter implies a role of visceral hypersensitivity in symptom generation in this subgroup of patients. However, these findings did not elucidate why some IBS patients are hypersensitive to artificially induced intestinal stimuli, while other IBS patients are not. Several pathophysiological factors associated with IBS, may affect nociception and thereby contribute to visceral hypersensitivity. Increased intestinal pain perception may result from mucosal inflammation, ${ }^{6-9}$ alterations in intestinal neuroendocrine activity, $^{10,11}$ or from intraluminal factors such as intestinal bacteria, their metabolic products or dietary compounds. ${ }^{12-14}$ The link between these intestinal biological processes and the development of visceral hypersensitivity has been investigated using animal models. ${ }^{13-15}$ However, data that focus on difference between patients with versus without visceral hypersensitivity are limited.

Previously, we found that faecal calprotectin, a marker of intestinal inflammation, ${ }^{16,17}$ and faecal chromogranin A ( $\mathrm{CgA})$, a neuroendocrine secretory protein of the enterochromaffin cells, ${ }^{18,19}$ were increased in IBS patients compared to healthy controls. In addition, faecal short chain fatty acids (SCFAs) and faecal human 8-defensin 2 (HBD2), as indicators of microbial activity and host-microbiome interaction, ${ }^{20-26}$ were decreased in IBS compared to healthy subjects. ${ }^{27}$ Furthermore, a decrease was found in IBS patients versus healthy subjects for plasma 5-hydroxyindoleacetic acid (5-HIAA), the principal metabolite of serotonin (5-hydroxitryptamine, 5-HT), ${ }^{11,28,29}$ and the ratio of 5HIAA and 5-HT. ${ }^{29}$ Yet, concentrations of $5-\mathrm{HT}$ were not altered in IBS versus controls. ${ }^{29}$ Neither were differences observed between these groups in concentrations of plasma citrulline, which is a marker of small intestinal effective enterocyte mass ${ }^{30,31}$ and may be seen as a surrogate marker of small bowel barrier function. ${ }^{32,33}$ Taken together, these findings point to alterations in biological processes, such as intestinal immune activation and neuroendocrine and microbial activity in IBS patients versus healthy controls. However, it is not known whether these markers also differ between IBS subgroups of normo- and hypersensitive subjects.

Aim of the present study was to provide more insight in biological processes associated with the presence of visceral hypersensitivity in IBS by comparing concentrations of individual biomarkers between IBS patients with and without visceral hypersensitivity. 
We hypothesize that concentrations of the measured biomarkers differ between visceral normo- and hypersensitive IBS patients and that these alterations may provide leads to mechanisms involved in hypersensitivity in IBS patients.

\section{Materials and methods}

The present study is part of a larger cohort study on the phenotypic and genotypic characterization of patients with IBS (Maastricht IBS cohort). The study protocol has been approved by the Maastricht University Medical Centre+ (MUMC+) Ethics Committee, was executed in compliance with the revised Declaration of Helsinki $\left(59^{\text {th }}\right.$ general assembly of the WMA, Seoul, South Korea, Oct. 2008), and was registered in the US National Library of Medicine (http://www.clinicaltrials.gov, NCT00775060).

\section{Study participants}

All study participants gave written informed consent prior to participation. Adult subjects, aged 18-75 years, diagnosed with IBS by a gastroenterologist based on the Rome III criteria, ${ }^{34}$ were recruited via the Gastroenterology-Hepatology outpatient department of the MUMC+, a secondary and tertiary referral centre. When considered indicated by the physician, GI endoscopy with biopsies, abdominal imaging by ultrasonography or CT scan, and/or blood, breath and faecal analyses were performed to exclude organic disease. History of abdominal surgery was considered an exclusion criterion, with the exception of appendectomy, laparoscopic cholecystectomy or hysterectomy. Rectal sensitivity was assessed in all IBS patients using an electronic rectal barostat (Distender II; G\&J Electronics, Toronto, ON, Canada, part: C7-CB-R) and a balloon of non-compliant material (Mui Scientific, Missisauga, ON, Canada, part: C7$2 \mathrm{CB}-\mathrm{R})$. The procedure was executed according to a standardized protocol, and a VASscore of $\geq 20 \mathrm{~mm}$ at pressure step $26 \mathrm{mmHg}$ was used as cut-off to define IBS patients being viscerally normo- $\left(\right.$ IBS $_{\text {NORM }}$ ) or hypersensitive to pain (IBS $S_{\mathrm{HP}}$ ), as described previously., ${ }^{4,5}$ All subjects completed several questionnaires as described in detail previously ${ }^{4}$ : i.e. Gl symptoms were assessed by the Gastrointestinal Symptom Rating Scale (GSRS) $)^{35,36}$ and a 14-day GI symptom diary, ${ }^{4,37}$ scores of anxiety and depression were assessed by the Hospital Anxiety and Depression Scale (HADS) ${ }^{38}$ and the State and Trait Anxiety Inventory (STAI), ${ }^{39}$ quality of life scores by the 36-item Short Form Health Survey (SF-36), ${ }^{40}$ and the use of medication by a general questionaire. ${ }^{4}$

\section{Analysis of biosamples}

Faecal samples were collected of all subjects, aliquoted and stored at $-80^{\circ} \mathrm{C}$ within 24 hours after defecation, until further analysis. Of all participants, fasting blood samples were collected from the antecubital vein using two precooled K2EDTA tubes. 
To avoid oxidative breakdown of platelets, $0,1 \mathrm{ml} \mathrm{1.4 \%} \mathrm{ascorbic} \mathrm{acid} \mathrm{(Sigma} \mathrm{Aldrich,} \mathrm{St.}$ Louis, MO) was added to one of the tubes, to obtain platelet poor plasma (PPP) samples after a centrifuge step. PPP samples were available for only a subgroup of study participants. K2EDTA tubes were centrifuged at $2300 \mathrm{~g}$ at $4^{\circ} \mathrm{C}$ for 10 minutes. Supernatants (i.e. plasma and/or PPP) were aliquoted and frozen at $-80^{\circ} \mathrm{C}$ until further analysis.

Faecal and plasma markers were selected based on literature to be measured as indicators of pathophysiological mechanisms in IBS. The markers were measured simultaneously and in blinded conditions by a commercial partner (Medische Laboratoria Dr. Stein \& Collegues, The Netherlands), to avoid additional thawing cycles. Calprotectin and human 6 -defensin 2 (HBD2) concentrations were determined using commercial enzyme-linked immunosorbent assay (ELISA, Bülmann, Switserland, ${ }^{19}$ and Immunodiagnostik AG, Germany, ${ }^{22}$ respectively), and chromogranin A using commercial radioimmunoassay (RIA, Euro Diagnostica, Sweden). ${ }^{19}$ All test kits were used according to manufacturers' instructions and the procedures were described in detail previously. $^{19,22}$ Short chain fatty acids (SCFAs), i.e. acetate, propionate, butyrate, valerate and caproate, were determined by gas chromatography-mass spectrometry (GCMS) according to the method described by Garciá-Villalba et al. ${ }^{41}$ Citrulline was measured in plasma samples by high pressure liquid chromatography (HPLC) fluorescence detection. ${ }^{31}$ Concentration of 5-HT and 5-HIAA were measured in PPP samples by a high-performance liquid chromatography (HPLC), as described previously. $^{42}$

\section{Data and statistical analysis}

Statistical analyses were performed using IBM SPSS Statistics version 22.0 (IBM Statistics for Windows, Armonk, New York). Data were checked for normality using a normal probability plot. Baseline characteristics are presented as mean $( \pm S D)$ or percentages when appropriate, for both IBS groups (i.e. IBS NORM and IBS $_{\text {HYP }}$ ). Dichotomous data were analysed using the $\mathrm{Chi}^{2}$ test and continuous (parametric) data using the independent t-test. In addition, the Spearman's rho was calculated to test the correlation between both the scores for abdominal pain as assessed by the end-of-day GI symptom diary (mean of 14-days) and the GSRS to VAS scores at the cut-off value of $26 \mathrm{mmHg}$ intra-balloon pressure of the rectal barostat.

To investigate differences in biomarker levels between the IBS ${ }_{\text {NORM }}$ and IBS HYP $_{\text {groups, }}$ the Mann Whitney $U$ test was used (due to a left skewed distribution of the biomarker data). A post-hoc analysis was conducted (linear regression analysis with natural log transformed biomarker data), to adjust the results for possible confounding effects of age, gender, IBS subtypes, symptoms of anxiety and depression, and medication use. Drugs that were included in the analysis were proton pump inhibitors (PPI), SSRIs, spasmolytics, prokinetics, anti-diarrheal drugs, laxatives, and non-steroid antiinflammatory drugs (NSAIDs). 


\section{Results}

In total, 167 IBS patients were included in the current study on biomarkers. Based on the outcome of the rectal barostat procedure $83(49.7 \%)$ patients were classified as viscerally hypersensitive (IBS ${ }_{\mathrm{HYP}}$ ) and $84(50.3 \%)$ as normosensitive (IBS NORM $_{\text {) }}$ to pain. The majority (i.e. $n=155,93 \%$ ) of the patients also participated in a previous study by our group. 4

For demographic characteristics see Table 6.1. In line with previous report, ${ }^{4}$ the $\mathrm{IBS}_{\mathrm{HYP}}$ patients were significantly younger, more often female and furthermore had a lower $\mathrm{BMI}$ compared to the $\mathrm{IBS}_{\text {NORM }}$ group $(\mathrm{p}<0.05)$. No differences were observed for IBS duration, i.e. time since diagnosis, or IBS subtype distribution between the groups. As GI symptom scores, scores for anxiety, depression and quality of life, life style factors and medication use of the majority of the included patients were described in detail in a previous study, ${ }^{4}$ we included these parameters as supplementary material (Supplementary Table S6.1) to the current study.

A correlation coefficient of $0.34(p<0.001)$ and $0.33(p<0.001)$ was found between the abdominal pain scores as assessed by the end-of-day diary and the GSRS, respectively, and the VAS scores at the cut-off value of $26 \mathrm{mmHg}$ intra-balloon pressure of the rectal barostat.

\section{Biomarker levels}

Faecal markers and plasma citrulline were measured in all 83 IBS $_{\text {HYP }}$ and 84 IBS NORM $_{\text {M }}$ patients, while 5-HT and 5-HIAA levels in PPP were available for only a subgroup of study participants, i.e. $53 \mathrm{IBS}_{\mathrm{HYP}}$ and $42 \mathrm{IBS}_{\mathrm{NORM}}$ patients (Table 6.2). All marker concentrations are presented as median $\left[Q_{1}-Q_{3}\right]$. For none of the markers a statistically significant difference was observed between the IBS $\mathrm{HYP}_{\text {}}$ and IBS $\mathrm{INORM}_{\text {Mroup. }}$ grou

Since patient characteristics or the use of specific drugs could influence the concentrations of the markers measured in the current study and potentially mask differences between the two IBS groups, a post hoc analysis was conducted to adjust for possible confounding effects of age, gender, BMI, IBS-subtype distribution, symptoms of anxiety and depression, and medication use. However, adjustment for these parameters (data not shown), did not lead to differences in marker levels with regard to the presence or absence of visceral hypersensitivity in IBS patients.

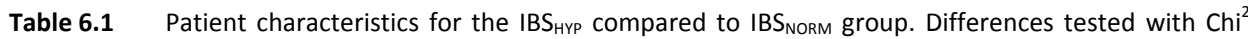
and independent t-test for categorical and continues data, respectively.

\begin{tabular}{lcc}
\hline Demographics and Lifestyle & $\mathrm{IBS}_{\mathrm{HYP}}(\mathrm{n}=83)$ & $\mathrm{IBS}_{\mathrm{NORM}}(\mathrm{n}=84)$ \\
\hline Age (years; mean $\pm \mathrm{SD})$ & $37.7 \pm 15.8^{* * *}$ & $46.7 \pm 16.3$ \\
Female sex (\%) & $81.9^{*}$ & 69.0 \\
$\mathrm{BMI}\left(\right.$ mean $\left.\mathrm{kg} / \mathrm{m}^{2} \pm \mathrm{SD}\right)$ & $23.5 \pm 4.5^{* *}$ & $25.7 \pm 4.3$ \\
$\mathrm{IBS}$ subtype: D / C / M / U (\%) & $26.5 / 24.1 / 42.2 / 7.2$ & $35.7 / 14.3 / 40.5 / 9.5$ \\
Duration of IBS symptoms (years; mean $\pm \mathrm{SD})$ & $13.2 \pm 12.2$ & $11.8 \pm 14.8$ \\
\hline
\end{tabular}

$\mathrm{p}<0.05, * * \mathrm{p}<0.01, * * * \mathrm{p}<0.001$ for IBS $\mathrm{HYP}$ Vs. IBS $\mathrm{INORM}$ 
Table 6.2 Levels of faecal (CgA, calprotectin, HBD2, SCFAs) and plasma markers (citrulline, 5-HT, 5-HIAA)

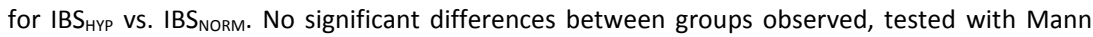
Whitney U test.

\begin{tabular}{|c|c|c|}
\hline Markers (median $\left[\mathrm{Q}_{1}-\mathrm{Q}_{3}\right]$ ) & $\begin{array}{l}I^{\prime B S} S_{H Y P} \\
(n=83) \\
\end{array}$ & $\begin{array}{c}I_{\text {IBS }} \text { NORM } \\
(n=84)\end{array}$ \\
\hline Chromogranin A (CgA) (nmol/g) & $14.5[7.4 ; 43.0]$ & $16.8[8.4 ; 47.9]$ \\
\hline Calprotectin ( $\mu \mathrm{g} / \mathrm{g})$ & $35.6[15.0 ; 72.0]$ & $33.5[16.7 ; 72.4]$ \\
\hline Human 8 -defensin 2 (HBD2) (ng/g) & $30.2[14.6 ; 51.7]$ & $25.9[15.8 ; 45.7]$ \\
\hline SCFA (C2): Acetate ( $\mu \mathrm{mol} / \mathrm{g})$ & $30.6[19.7 ; 45.5]$ & $30.6[20.6 ; 45.4]$ \\
\hline SCFA (C3): Propionate ( $\mu \mathrm{mol} / \mathrm{g})$ & $8.8[5.1 ; 13.8]$ & $9.2[5.7 ; 13.4]$ \\
\hline SCFA (C4): Butyrate ( $\mu \mathrm{mol} / \mathrm{g})$ & $7.3[4.1 ; 12.7]$ & $7.9[4.6 ; 12.2]$ \\
\hline SCFA (C5): Valerate ( $\mu \mathrm{mol} / \mathrm{g})$ & $1.2[0.8 ; 1.6]$ & $1.3[0.8 ; 1.9]$ \\
\hline SCFA (C6): Caproate ( $\mu \mathrm{mol} / \mathrm{g})$ & $0.09[0.02 ; 0.63]$ & $0.05[0.02 . ; 0.45]$ \\
\hline \multirow[t]{2}{*}{ Plasma citrulline $(\mu \mathrm{mol} / \mathrm{l})$} & $39.8[34.4 ; 49.3]$ & $41.9[33.5 ; 50.2]$ \\
\hline & $\begin{array}{l}\mathrm{IBS}_{\mathrm{HYP}} \\
(n=53) \\
\end{array}$ & $\begin{array}{c}\mathrm{IBS}_{\text {NORM }} \\
(\mathrm{n}=42)\end{array}$ \\
\hline 5-HT $(\mu \mathrm{g} / \mathrm{l})$ & $3.6[1.7 ; 9.2]$ & $4.6[1.7 ; 8.4]$ \\
\hline 5-HIAA ( $\mu \mathrm{g} / \mathrm{I})$ & $17.8[12.3 ; 28.0]$ & $22.1[14.9 ; 35.4]$ \\
\hline 5-HIAA / 5-HT ratio & $5.0[1.8 ; 10.5]$ & $6.2[2.2 ; 11.1]$ \\
\hline
\end{tabular}

\section{Discussion}

In the current study, we compared IBS patients with and without visceral hypersensitivity with regard to faecal and plasma biomarkers for pathophysiological processes that may be associated with altered visceral perception. In contrast to our hypothesis, none of the tested markers were significantly different in the hypersensitive compared to the normosensitive IBS group.

Abdominal pain is a highly prevalent symptom in IBS and pain characteristics are among the major Rome III diagnostic criteria for IBS. ${ }^{34}$ In line with previous studies ${ }^{43,44}$ we have observed a highly significant but moderate correlation $(r=0.3, p<0.001)$ between increased visceral pain perception and abdominal pain scores in IBS patients. A subgroup of IBS patients (49.7\%) was found to have increased visceral perception to an artificial stimulus induced by rectal balloon distention, which is in accordance with findings by others. ${ }^{1,2,5}$ It indicates that increased visceral perception may contribute to symptom generation in about half of the IBS population and may have relevance for underlying pathophysiology. We hypothesized that factors that potentially affect visceral pain perception, such as intestinal inflammation or dysregulation of the neuroendocrine or microbiota activity, may be altered in IBS patients with visceral hypersensitivity when compared to normosensitive patients.

A low grade immune activation has previously been associated with IBS, and it may influence the process of nociception. Products of intestinal immune cells and epithelial 
cells, involved in this process are cytokines, chemokines, prostanoids, proteases, bradykinins, biogenic amines (e.g. histamine and 5-HT), neuro-peptides (e.g. substance $\mathrm{P}$, somatostatin), reductions in $\mathrm{pH}$ and increases in ATP. ${ }^{6-9,15,45-47}$ In previous studies, calprotectin has been found to be slightly though significantly increased in IBS patients compared to healthy controls. ${ }^{27,47}$ In the present study we measured this faecal protein as a non-invasive marker of intestinal inflammation and tested the relation to visceral pain perception within the IBS population. However, in our IBS patient population calprotectin concentrations could not provide leads for an explanation for the difference in visceroperception in IBS patients. This does not exclude a role for intestinal inflammation as factor contributing to hypersensitivity in IBS. Other inflammatory factors, not measured in the present study, may contribute to visceral hypersensitivity. For example, mucosal mast cells were found to be associated with the stimulation of visceral neural signalling in IBS and mast cell mediators such as histamine and proteases were found to be increased in mucosal biopsies of these patients, ${ }^{45}$ but have not been assessed in the current study.

Serotonin (5-HT) is known to modulate several intestinal processes such as motility, secretion and perception. ${ }^{10,11}$ Most 5-HT is produced by enterochromaffin cells and can activate intrinsic or extrinsic primary afferent nerves in the intestinal mucosa. ${ }^{48}$ Previous studies have suggested that elevated serotonin activity may act as a excitatory mediator in visceral sensory pathways, in particular via increased activation of $5-\mathrm{HT}_{3}$ receptor. ${ }^{49}$ Moreover, treatment with a $5-\mathrm{HT}_{3}$ receptor antagonist has resulted in higher perception thresholds to colonic distension in IBS patients. ${ }^{49}$ Recently, in an intervention study Keszthelyi and colleagues have demonstrated that oral administration of 5-hydroxytryptophan (5-HTP), a direct precursor of 5-HT, induced rectal hyperalgesia in hypersensitive but not in normosensitive IBS patients. Furthermore the intake of 5-HTP was accompanied by an increase in blood plasma 5-HIAA levels, while it did not affect the 5-HT plasma levels. ${ }^{25}$ Based on these findings we hypothesised that $5-\mathrm{HT}$ levels will not be altered in hypersensitive compared to normosensitive IBS patients, but that hypersensitive subjects may be characterised by increased 5-HIAA levels in plasma. However, in the current study for both markers no differences were observed between the IBS subgroups. Which may indicate, that in contrast to findings in an acute intervention study with 5-HTP, baseline plasma levels of 5-HT and 5-HIAA do not provide an explanation for differences in visceral sensitivity between IBS patients. Plasma serotonin levels are thought to represent mucosal availability of 5-HT. However, it should be acknowledged that differences between hyper- and normosensitive IBS patients in mucosal serotonin activity, which were not assessed in the current study, cannot be excluded.

Hypersensitivity to colonic distension in IBS patients can be transferred to rats via faecal transplants, ${ }^{13}$ indicating that luminal factors, such as the microbiota, its toxins or metabolites, or dietary products, may alter the visceral sensitivity of these rodents. Furthermore, Rousseaux et al. found that specific Lactobabacilli species (i.e L. 
acidophilus NCFM) were able to affect $\mu$-opiod and cannabinoid receptors in intestinal epithelial cells of rats, suggesting that the intestinal microbiota may influence visceral perception. ${ }^{50}$ We measured faecal concentrations of HBD2, a human antimicrobial peptide produced by intestinal epithelial cells in response to bacteria. ${ }^{23}$ Alterations in microbiota composition may result in changes in the intestinal mucosa response towards bacteria, possibly via HBD2. In previous studies both increased and decreased faecal HBD2 has been observed in IBS patients compared to healthy controls. ${ }^{22,27}$ However, we did not observe any differences in HBD2 faecal concentrations between the hyper- and normosensitive IBS group. We also measured several SCFAs in faecal samples. These SCFAs are products of microbial fermentation of non-digested oligosaccharides in the colon and are generally regarded as beneficial for gut health. ${ }^{20,21}$ SCFAs are presumed to be a major player in maintenance of gut and immune

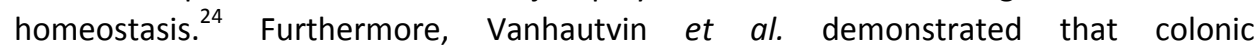
administration of butyrate, at physiologically relevant concentrations, dosedependently decreases visceral sensitivity in healthy volunteers. ${ }^{26}$ The intraluminal SCFA concentration is affected by microbiota composition as well as dietary substrates. Our study demonstrates no significant differences in faecal SCFA concentrations between the IBS groups, which indicates that alterations in faecal SCFA levels are not associated with the presence of hypersensitivity in IBS patients. The intestinal microbiota composition itself and the dietary pattern of the study subjects were not investigated in the current study.

We previously reported that several markers (i.e. calprotectin, CgA, HBD2, the SCFAs valerate and caproate, 5-HIAA and the 5-HIAA/5-HT ratio) were significantly altered in IBS patients compared to healthy subjects. ${ }^{27,29}$ However, in the current study no differences were observed between the hyper- and normosensitive IBS patients with regard to these markers. Thereby, intestinal biological processes, related to the tested markers, are associated with the presence of IBS, but are not likely to play a prominent role in the development or maintenance of visceral hypersensitivity in these patients. It should be taken into account that the panel of tested markers is indicative for only some of the key biological processes, which does not exclude that other factors contribute to the presence of visceral hypersensitivity in IBS. These factors include for example mucosal mast cell activation or mucosal cytokine release, ${ }^{15,45,51}$ alterations in trans-nociceptive transducer molecules (e.g. transient receptor potential (TRP) and acid-sensing ion channels (ASIC)), ${ }^{6,52}$ dysfunctional intestinal barrier function, ${ }^{53,54}$ and/or alterations in microbial composition, ${ }^{12,14}$ and mucosal serotonin metabolism. ${ }^{55}$ In line with the general hypothesis that the interaction between the brain and gut is involved in the development of visceral hypersensitivity in IBS, future studies should focus on dysregulation of mechanisms on intestinal level in combination with altered central pain modulation and/or with psychological factors, such as stress, anxiety, 
depression or somatization. ${ }^{56-58}$ Further research is needed to elucidate this complex interplay between intestinal and central factors in the target population.

\section{Conclusion}

In the current study, no significant differences were found between hypersensitive and normosensitive IBS patients with regard to the tested biomarkers: i.e. faecal calprotectin, chromogranin $A$, human $\beta$-defensin 2 , and short chain fatty acids, and plasma levels of 5-HT, 5-HIAA, and citrulline. The set of markers we explored represents specific pathophysiological mechanisms in IBS, such as immune activation, neuroendocrine and microbial activity. Although further research is needed including central and peripheral neural factors, our findings indicate that these pathophysiological mechanisms do not play a major role in the presence of visceral hypersensitivity in IBS patients. 


\section{References}

1. Ritchie J. Pain from distension of the pelvic colon by inflating a balloon in the irritable colon syndrome. Gut. 1973;14:125-32.

2. Mertz H, Naliboff B, Munakata J, Niazi N, Mayer EA. Altered rectal perception is a biological marker of patients with irritable bowel syndrome. Gastroenterology. 1995;109:40-52.

3. Piche $M$, Arsenault $M$, Poitras $P$, Rainville $P$, Bouin $M$. Widespread hypersensitivity is related to altered pain inhibition processes in irritable bowel syndrome. Pain. 2010;148:49-58.

4. Ludidi S, Mujagic Z, Jonkers D, Keszthelyi D, Hesselink M, Kruimel J, et al. Markers for visceral hypersensitivity in patients with irritable bowel syndrome. Neurogastroenterol Motil. 2014;26:1104-11.

5. Ludidi S, Conchillo JM, Keszthelyi D, Van Avesaat M, Kruimel JW, Jonkers DM, et al. Rectal hypersensitivity as hallmark for irritable bowel syndrome: defining the optimal cutoff. Neurogastroenterol Motil. 2012;24:729-33, e345-6.

6. Knowles $\mathrm{CH}$, Aziz Q. Basic and clinical aspects of gastrointestinal pain. Pain. 2009;141:191-209.

7. Basbaum Al, Bautista DM, Scherrer G, Julius D. Cellular and molecular mechanisms of pain. Cell. 2009;139:267-84.

8. Keszthelyi D, Troost FJ, Simren M, Ludidi S, Kruimel JW, Conchillo JM, et al. Revisiting concepts of visceral nociception in irritable bowel syndrome. Eur J Pain. 2012;16):1444-54.

9. Keszthelyi D, Troost FJ, Jonkers DM, Helyes Z, Hamer HM, Ludidi S, et al. Alterations in mucosal neuropeptides in patients with irritable bowel syndrome and ulcerative colitis in remission: a role in pain symptom generation? Eur J Pain. 2013;17:1299-306.

10. Gilet M, Eutamene H, Han H, Kim HW, Bueno L. Influence of a new 5-HT4 receptor partial agonist, YKP10811, on visceral hypersensitivity in rats triggered by stress and inflammation. Neurogastroenterol Motil. 2014;26:1761-70.

11. Keszthelyi D, Troost FJ, Masclee AA. Understanding the role of tryptophan and serotonin metabolism in gastrointestinal function. Neurogastroenterol Motil. 2009;21:1239-49.

12. Rajilic-Stojanovic M, Jonkers DM, Salonen A, Hanevik K, Raes J, Jalanka J, et al. Intestinal Microbiota And Diet in IBS: Causes, Consequences, or Epiphenomena? Am J Gastroenterol. 2015;110:278-87.

13. Crouzet L, Gaultier E, Del'Homme C, Cartier C, Delmas E, Dapoigny M, et al. The hypersensitivity to colonic distension of IBS patients can be transferred to rats through their fecal microbiota. Neurogastroenterol Motil. 2013;25:e272-82.

14. Kannampalli P, Pochiraju S, Chichlowski M, Berg BM, Rudolph C, Bruckert M, et al. Probiotic Lactobacillus rhamnosus GG (LGG) and prebiotic prevent neonatal inflammation-induced visceral hypersensitivity in adult rats. Neurogastroenterol Motil. 2014;26:1694-704.

15. Stanisor OI, van Diest SA, Yu Z, Welting O, Bekkali N, Shi J, et al. Stress-induced visceral hypersensitivity in maternally separated rats can be reversed by peripherally restricted histamine-1-receptor antagonists. PloS One. 2013;8:e66884.

16. Lundberg JO, Hellstrom PM, Fagerhol MK, Weitzberg E, Roseth AG. Technology insight: calprotectin, lactoferrin and nitric oxide as novel markers of inflammatory bowel disease. Nat Clin Pract Gastroenterol Hepatol. 2005;2:96-102.

17. Wassell J, Dolwani S, Metzner M, Losty H, Hawthorne A. Faecal calprotectin: a new marker for Crohn's disease? Ann Clin Biochem. 2004;41:230-2.

18. Usellini L, Finzi G, Riva C, Capella C, Mochizuki T, Yanaihara C, et al. Ultrastructural identification of human secretin cells by the immunogold technique. Their costorage of chromogranin A and serotonin. Histochemistry. 1990;94:113-20.

19. Ohman L, Stridsberg M, Isaksson S, Jerlstad P, Simren M. Altered levels of fecal chromogranins and secretogranins in IBS: relevance for pathophysiology and symptoms? Am J Gastroenterol. 2012;107: 440-7.

20. Hamer HM, Jonkers D, Venema K, Vanhoutvin S, Troost FJ, Brummer RJ. Review article: the role of butyrate on colonic function. Aliment Pharmacol Ther. 2008;27:104-19.

21. Tana C, Umesaki Y, Imaoka A, Handa T, Kanazawa M, Fukudo S. Altered profiles of intestinal microbiota and organic acids may be the origin of symptoms in irritable bowel syndrome. Neurogastroenterol Motil. 2010;22:512-9, e114-5. 
22. Langhorst J, Junge A, Rueffer A, Wehkamp J, Foell D, Michalsen A, et al. Elevated human beta-defensin-2 levels indicate an activation of the innate immune system in patients with irritable bowel syndrome. Am J Gastroenterol. 2009;104:404-10.

23. Vora P, Youdim A, Thomas LS, Fukata M, Tesfay SY, Lukasek K, et al. Beta-defensin-2 expression is regulated by TLR signaling in intestinal epithelial cells. J Immunol. 2004;173:5398-405.

24. Tan J, McKenzie C, Potamitis M, Thorburn AN, Mackay CR, Macia L. The role of short-chain fatty acids in health and disease. Adv Immunol. 2014;121:91-119.

25. Keszthelyi D, Troost FJ, Jonkers DM, van Eijk HM, Dekker J, Buurman WA, et al. Visceral hypersensitivity in irritable bowel syndrome: evidence for involvement of serotonin metabolism - a preliminary study. Neurogastroenterol Motil. 2015.

26. Vanhoutvin SA, Troost FJ, Kilkens TO, Lindsey PJ, Hamer HM, Jonkers DM, et al. The effects of butyrate enemas on visceral perception in healthy volunteers. Neurogastroenterol Motil. 2009;21:952-e76.

27. Mujagic Z, Tigchelaar F, Zhernakova A, Ludwig T, Ramiro-Garcia J, Baranska A, et al. A novel gut health biomarker panel for irritable bowel syndrome and the application in the general population. 2015.

28. Atkinson W, Lockhart S, Whorwell PJ, Keevil B, Houghton LA. Altered 5-hydroxytryptamine signaling in patients with constipation- and diarrhea-predominant irritable bowel syndrome. Gastroenterology. 2006;130:34-43.

29. Thijssen AY, Mujagic Z, Jonkers D, Ludidi S, Keszthelyi D, Hesselink M, et al. Plasma 5-hydroxytryptamine and 5-hydroxyindole acetic acid levels: indicators for altered serotonin metabolism in IBS? 2015.

30. Windmueller HG, Spaeth AE. Source and fate of circulating citrulline. Am J Physiol. 1981;241:E473-80.

31. Crenn P, Messing B, Cynober L. Citrulline as a biomarker of intestinal failure due to enterocyte mass reduction. Clin Nutr. 2008;27:328-39.

32. Lutgens LC, Blijlevens NM, Deutz NE, Donnelly JP, Lambin P, de Pauw BE. Monitoring myeloablative therapy-induced small bowel toxicity by serum citrulline concentration: a comparison with sugar permeability tests. Cancer. 2005;103:191-9.

33. van Vliet MJ, Tissing WJ, Rings EH, Koetse HA, Stellaard F, Kamps WA, et al. Citrulline as a marker for chemotherapy induced mucosal barrier injury in pediatric patients. Pediatr Blood Cancer. 2009;53: 1188-94.

34. Longstreth GF, Thompson WG, Chey WD, Houghton LA, Mearin F, Spiller RC. Functional bowel disorders. Gastroenterology. 2006;130:1480-91.

35. Svedlund J, Sjodin I, Dotevall G. GSRS--a clinical rating scale for gastrointestinal symptoms in patients with irritable bowel syndrome and peptic ulcer disease. Dig Dis Sci. 1988;33:129-34.

36. Revicki DA, Wood M, Wiklund I, Crawley J. Reliability and validity of the Gastrointestinal Symptom Rating Scale in patients with gastroesophageal reflux disease. Qual Life Res. 1998;7:75-83.

37. Thijssen AY, Jonkers DM, Leue C, van der Veek PP, Vidakovic-Vukic M, van Rood YR, et al. Dysfunctional cognitions, anxiety and depression in irritable bowel syndrome. J Clin Gastroenterol. 2010;44:e236-41.

38. Zigmond AS, Snaith RP. The hospital anxiety and depression scale. Acta Psychiatr Scand. 1983;67: 361-70.

39. Spielberger SC. State-trait Anxiety Inventory. Consulting Psychologists Press. 1970.

40. Creed F, Ratcliffe J, Fernandez L, Tomenson B, Palmer S, Rigby C, et al. Health-related quality of life and health care costs in severe, refractory irritable bowel syndrome. Ann Intern Med. 2001;134:860-8.

41. Garcia-Villalba R, Gimenez-Bastida JA, Garcia-Conesa MT, Tomas-Barberan FA, Carlos Espin J, Larrosa M. Alternative method for gas chromatography-mass spectrometry analysis of short-chain fatty acids in faecal samples. J Sep Scie. 2012;35:1906-13.

42. Hirowatari $\mathrm{Y}$, Hara K, Kamihata H, Iwasaka T, Takahashi H. High-performance liquid chromatographic method with column-switching and post-column reaction for determination of serotonin levels in platelet-poor plasma. Clin Biochem. 2004;37:191-7.

43. Kanazawa M, Palsson OS, Thiwan SI, Turner MJ, van Tilburg MA, Gangarosa LM, et al. Contributions of pain sensitivity and colonic motility to IBS symptom severity and predominant bowel habits. Am J Gastroenterol. 2008;103:2550-61.

44. Posserud I, Syrous A, Lindstrom L, Tack J, Abrahamsson H, Simren M. Altered rectal perception in irritable bowel syndrome is associated with symptom severity. Gastroenterology. 2007;133:1113-23. 
45. Barbara G, Stanghellini V, De Giorgio R, Cremon C, Cottrell GS, Santini D, et al. Activated mast cells in proximity to colonic nerves correlate with abdominal pain in irritable bowel syndrome. Gastroenterology. 2004;126:693-702.

46. Di Nardo G, Barbara G, Cucchiara S, Cremon C, Shulman RJ, Isoldi S, et al. Neuroimmune interactions at different intestinal sites are related to abdominal pain symptoms in children with IBS. Neurogastroenterol Motil. 2014;26:196-204.

47. Dolwani S, Metzner M, Wassell JJ, Yong A, Hawthorne AB. Diagnostic accuracy of faecal calprotectin estimation in prediction of abnormal small bowel radiology. Aliment Pharmacol Ther. 2004;20:615-21.

48. Gershon MD. Nerves, reflexes, and the enteric nervous system: pathogenesis of the irritable bowel syndrome. J Clin Gastroenterol. 2005;39(5 Suppl 3):S184-93.

49. Delvaux M, Louvel D, Mamet JP, Campos-Oriola R, Frexinos J. Effect of alosetron on responses to colonic distension in patients with irritable bowel syndrome. Aliment Pharmacol Ther. 1998;12:849-55.

50. Rousseaux C, Thuru X, Gelot A, Barnich N, Neut C, Dubuquoy L, et al. Lactobacillus acidophilus modulates intestinal pain and induces opioid and cannabinoid receptors. Nat Med. 2007;13:35-7.

51. Buckley MM, O'Halloran KD, Rae MG, Dinan TG, O'Malley D. Modulation of enteric neurons by interleukin-6 and corticotropin-releasing factor contributes to visceral hypersensitivity and altered colonic motility in a rat model of irritable bowel syndrome. J Physiol. 2014;592:5235-50.

52. Holzer P. Acid sensing by visceral afferent neurones. Acta Physiol. 2011;201:63-75.

53. Mujagic Z, Ludidi S, Keszthelyi D, Hesselink MA, Kruimel JW, Lenaerts K, et al. Small intestinal permeability is increased in diarrhoea predominant IBS, while alterations in gastroduodenal permeability in all IBS subtypes are largely attributable to confounders. Aliment Pharmacol Ther. 2014;40:288-97.

54. Zhou Q, Zhang B, Verne GN. Intestinal membrane permeability and hypersensitivity in the irritable bowel syndrome. Pain. 2009;146:41-6.

55. Keszthelyi D, Troost FJ, Jonkers DM, Kruimel JW, Leue C, Masclee AA. Decreased levels of kynurenic acid in the intestinal mucosa of IBS patients: relation to serotonin and psychological state. J Psychosom Res. 2013;74:501-4.

56. Elsenbruch S. Abdominal pain in Irritable Bowel Syndrome: a review of putative psychological, neural and neuro-immune mechanisms. Brain Behav Immun. 2011;25:386-94.

57. Schmid J, Langhorst J, Gass F, Theysohn N, Benson S, Engler H, et al. Placebo analgesia in patients with functional and organic abdominal pain: a fMRI study in IBS, UC and healthy volunteers. Gut. 2015;64:418-27.

58. Arsie E, Coletta M, Cesana BM, Basilisco G. Symptom-association probability between meal ingestion and abdominal pain in patients with irritable bowel syndrome. Does somatization play a role? Neurogastroenterol Motil. 2015;27:416-22. 


\section{Supplementary table}

Gastrointestinal (GI) symptoms assessed by a 15-day GI symptom diary (presented as mean of 14 days \pm SD), based on a 1-to-5 point scale, and the Gastrointestinal Symptom Rating Scale (GSRS), based on a 1-to-7 point scale (presented for 5 subscales/syndromes). Symptoms of anxiety and depression assessed by the Hospital Anxiety and Depression Scale (HADS) and presented as mean \pm SD for the depression and anxiety subscale (range 0-21). Trait anxiety score as assessed by the State and Trait Anxiety Inventory (STAI) presented as mean \pm SD (range 0-100). Composite scores for mental and physical quality of life of the SF-36 questionnaire presented as mean \pm SD (range 0-100). Use of medication, at least once in the 14 days prior to inclusion (selfreported and cross-checked in medical files) presented as \% of users per group. The variable 'use of any medication' includes the listed drugs, but also any other medication (such as statins, oral anti-conceptive drugs, etc.).

Table S6.1 Viscerally hypersensitive (IBS ${ }_{\text {HYP }}$ ) compared to normosensitive $\left(\right.$ IBS $\left._{\text {NORM }}\right)$ IBS patients.

\begin{tabular}{|c|c|c|}
\hline Parameters (mean $\pm \mathrm{SD}$ ) & $\mathrm{IBS}_{\mathrm{HYP}}(\mathrm{n}=83)$ & $\operatorname{IBS}_{\text {NORM }}(n=84)$ \\
\hline \multicolumn{3}{|c|}{ 14-day End-of-Day GI symptom diary (1-to-5 point scale) } \\
\hline Abdominal pain & $2.7 \pm 0.8^{* * *}$ & $2.1 \pm 0.8$ \\
\hline Abdominal discomfort & $2.8 \pm 0.7 * * *$ & $2.3 \pm 0.8$ \\
\hline Bloating & $2.5 \pm 0.9 * *$ & $2.0 \pm 1.0$ \\
\hline Belching & $1.8 \pm 0.8$ & $1.6 \pm 0.7$ \\
\hline Nausea & $1.8 \pm 0.9$ & $1.6 \pm 0.8$ \\
\hline Flatulence & $2.4 \pm 0.9$ & $2.3 \pm 0.9$ \\
\hline Constipation & $1.8 \pm 0.9 * * *$ & $1.3 \pm 0.4$ \\
\hline Diarrhoea & $2.8 \pm 0.7$ & $2.3 \pm 0.8$ \\
\hline \multicolumn{3}{|c|}{ Gastrointestinal Symptom Rating Scale syndromes (1-to-7 point scale) } \\
\hline Abdominal pain & $3.5 \pm 1.2$ & $3.2 \pm 1.2$ \\
\hline Reflux & $2.2 \pm 1.2^{*}$ & $1.8 \pm 1.2$ \\
\hline Indigestion & $4.1 \pm 1.3^{*}$ & $3.7 \pm 1.2$ \\
\hline Constipation & $3.7 \pm 1.6^{* * *}$ & $3.0 \pm 1.2$ \\
\hline Diarrhoea & $3.5 \pm 1.5$ & $3.5 \pm 1.5$ \\
\hline \multicolumn{3}{|l|}{ Psychological symptoms (mean \pm SD) } \\
\hline HADS depression scale & $5.3 \pm 4.2^{*}$ & $4.1 \pm 3.5$ \\
\hline HADS anxiety scale & $7.2 \pm 3.6$ & $6.8 \pm 4.0$ \\
\hline STAI trait anxiety scale & $41.0 \pm 12.3$ & $40.3 \pm 10.9$ \\
\hline \multicolumn{3}{|l|}{ SF-36 QoL compositie scores (mean \pm SD) } \\
\hline SF-36 mental QoL score & $45.9 \pm 10.7$ & $47.2 \pm 10.9$ \\
\hline SF-36 physical QoL score & $38.5 \pm 10.0$ & $41.7 \pm 10.3$ \\
\hline \multicolumn{3}{|l|}{ Life style factors (\%) } \\
\hline Current or previous smoker & 31.3 & 21.1 \\
\hline Alcohol abstainers: 0 units/week & 50.6 & 35.7 \\
\hline Moderate alcohol use: 1-15 units/week & 44.6 & 52.4 \\
\hline \multicolumn{3}{|l|}{ Use of medication in 14 days prior to inclusion (\%) } \\
\hline Proton Pump Inhibitors (PPI) & 25.3 & 30.1 \\
\hline Selective Serotonin Reuptake Inhibitors (SSRI) & $21.5^{*}$ & 12.3 \\
\hline Laxatives & 20.3 & 20.5 \\
\hline Spasmolytic drugs & 6.3 & 12.3 \\
\hline Prokinetic drugs & 3.8 & 2.7 \\
\hline Anti-diarrheal drugs & 3.8 & 2.7 \\
\hline Non Steroid Anti Inflammatory Drugs (NSAID) & 12.7 & 13.7 \\
\hline Use of any medication & 77.6 & 81.9 \\
\hline
\end{tabular}

Differences tested with $\mathrm{Chi}^{2}$ and Student t-test for categorical and continues data, respectively. ${ }^{*} \mathrm{p}<0.05$; $* * \mathrm{p}<0.01, * * * \mathrm{p}<0.001$ 
Biomarkers for visceral hypersensitivity in IBS 


\section{Chapter}

\section{Serotonin metabolism in IBS}

Alterations in serotonin metabolism in Irritable Bowel Syndrome

Annemieke Y Thijssen*, Zlatan Mujagic*, Daisy MAE Jonkers, Samefko Ludidi, Daniel Keszthelyi, Martine AM Hesselink, Cees M Clemens, José M Conchillo, Joanna W Kruimel, Ad AM Masclee

* Both authors contributed equally to this manuscript

Alimentary Pharmacology \& Therapeutics, 2015 Nov 5. [Epub ahead of print] 


\section{Abstract}

\section{Background and aims}

Alterations in serotonin (5-HT) metabolism have been postulated to play a role in the pathogenesis of Irritable Bowel Syndrome (IBS). However, previous reports regarding 5-HT metabolism in IBS are contradicting. The aim of this study was to compare platelet poor plasma (PPP) 5-HT and 5-hydroxyindole acetic acid (5-HIAA) levels and their ratio in a large cohort of IBS patients and healthy controls (HC), including IBS- subgroup analysis.

\section{Methods}

IBS patients and HCs were evaluated for fasting PPP 5-HT and 5-HIAA levels. Furthermore, GI-symptom diary, GSRS, quality of life, anxiety and depression scores were assessed in the two weeks before blood sampling.

\section{Results}

154 IBS patients and $137 \mathrm{HC}$ were included. No differences were detected in plasma 5-HT between groups. The 5-HIAA concentrations and 5-HIAA/5-HT ratio were significantly lower in IBS compared to HC: $24.6 \pm 21.9$ vs. $39.0 \pm 29.5 \mathrm{ug} / \mathrm{l}(\mathrm{p}<0.001)$ and $8.4 \pm 12.2$ vs. $13.5 \pm 16.6$ ( $p<0.01$ ), respectively. Subtype analysis for 5 -HIAA showed all subtypes to be significantly different from HC. The 5-HIAA/5-HT ratio was significantly lower in the IBS-M subtype versus HC. Linear regression analysis points to an influence of gender but not of Gl-symptoms, psychological scores or medication use.

\section{Conclusion}

We demonstrated that fasting 5-HT plasma levels are not significantly different in IBS patients compared to controls. However, decreased 5-HIAA levels and 5-HIAA/5-HT ratio in IBS patients may reflect altered serotonin metabolism in IBS. Gender affects 5HIAA levels in IBS patients, but no effects of drugs, such as SSRIs, or higher GI-symptom or psychological scores were found. 


\section{Introduction}

Irritable Bowel Syndrome (IBS) is a highly prevalent disorder with up to $15 \%$ of the Western population affected and a female predominance. ${ }^{1,2}$ The Rome III criteria offer support in diagnosing IBS $^{3,4}$ with further subtyping based on predominant bowel habits. ${ }^{5}$ The pathophysiology of IBS is still incompletely understood. ${ }^{6}$ An important role has been claimed for dysregulations along the gut-brain axis. ${ }^{7}$ Serotonin (5-hydroxytryptamine, 5-HT) as neurotransmitter ${ }^{7-9}$ not only modulates physiological and behavioral processes in the brain ${ }^{10}$ but is also a key factor in the enteric nervous system. ${ }^{11}$ Moreover, $5-\mathrm{HT}$ is an important paracrine signaling molecule in the mucosal sensory transduction in the gut. ${ }^{11}$ Although the physiological role of $5-\mathrm{HT}$ in the gastrointestinal (GI) tract is complex and is not completely elucidated, serotonin has been shown to be involved in the regulation of secretion, motility and perception in the gut. $^{11,12}$

The enterochromaffin (EC) cells in the intestinal epithelium produce $90-95 \%$ of total 5-HT in the human body. ${ }^{11-13} 5-\mathrm{HT}$ is synthesized from tryptophan by tryptophan hydroxylase and aromatic amino acid decarboxylase. Upon release, 5-HT is inactivated via uptake by the serotonin reuptake transporter (SERT) located among others in the epithelial cells lining the gut lumen ${ }^{7,12,14}$ and broken down by among others the intracellular molecule monoamine oxidase (MAO) into 5-hydroxy indoleacetic acid (5-HIAA), which is excreted in urine. ${ }^{11,15,16}$ Decreased SERT activity results in decreased 5-HT uptake and therefore decreased 5-HIAA production. ${ }^{17}$ Systemic 5-HT is believed to be primarily derived from the EC cells ${ }^{16}$ and is rapidly taken up by platelets, which consequently contain the majority of serotonin in peripheral blood. ${ }^{10,18}$ Platelet poor plasma (PPP) levels of 5-HT and 5-HIAA are considered to be surrogate markers of intestinal 5-HT metabolism. ${ }^{8,11,18}$

Previous studies have explored serotonin metabolism in IBS, but results are difficult to interpret and contradicting. Increased plasma 5-HT levels have been found in IBS, ${ }^{8,19-21}$ both in fasting and postprandial conditions, while others did not find a difference in fasting 5-HT levels compared to controls or levels were below detection threshold. ${ }^{20,22}$ With respect to plasma 5-HIAA, the principal metabolite of 5-HT, Atkinson et al. noted that 5-HIAA levels were slightly decreased in IBS-C. ${ }^{8}$ The 5-HIAA/5-HT ratio, representing turnover of serotonin, was found to be decreased in diarrhea predominant IBS patients. ${ }^{19,20}$ It should be acknowledged that data on 5-HT release and activity is influenced by a number of variables: e.g. fasting or (meal) stimulated condition, expression and activity of SERT, reuptake of serotonin within the gut and transport of serotonin in liver, lungs and platelets ${ }^{11}$ and presence of concurrent psychopathology in IBS patients ${ }^{23-25}$ such as anxiety and depression. ${ }^{18,26,27}$ Interpretation of published data on human serotonin metabolism in IBS is further hampered by factors such as sample processing, the analytical technique used, small 
sample size studies, the heterogeneous nature of IBS, imbalance in distribution among IBS subtypes, sampling in fasting versus postprandial state, presence of psychopathology and use of drugs prescribed for IBS that modify gastrointestinal function or the 5 -HT system. ${ }^{28}$ Large studies are needed, preferably from well characterized IBS patient groups that assess parameters of 5-HT metabolism taking into account IBS subtypes and potential confounding factors.

We hypothesized that (i) plasma 5-HT levels and 5-HIAA concentrations are altered in IBS patients compared to healthy controls, and that this (ii) depends on the IBS subtype and (iii) is related to more severe abdominal symptoms and to altered anxiety and depression scores. The primary aim of this study was therefore to compare platelet poor plasma 5-HT and 5-HIAA levels and their ratio in a large cohort of wellcharacterized IBS patients and healthy controls. The secondary aim was to compare differences in these parameters between subgroups of IBS patients based on IBS subtypes and severity of abdominal and psychological symptoms.

\section{Materials and methods}

The present study is part of a larger cohort study on the phenotypical and genotypical characterization of patients with IBS and healthy controls, the Maastricht IBS cohort, wherein multiple parameters were assessed according to standardized procedures. Patients and controls that handed in a blood sample for assessment of serotonin and 5HIAA were included in the current study. All subjects have given written informed consent prior to their inclusion. The study was approved by the Medical Ethics Committee of the Maastricht University Medical Centre+ (MUMC+) and executed according to the revised Declaration of Helsinki $\left(59^{\text {th }}\right.$ general assembly of the WMA, Seoul, South Korea, Oct. 2008). The study has been registered at the US National Library of Medicine (http://www.clinicaltrials.gov NCT00775060).

\section{Study participants}

From February 2009 until present time, patients with IBS between 18-75 years of age, fulfilling Rome III criteria, are recruited via the outpatient clinic of the department of Gastroenterology-Hepatology of MUMC+ and via general practitioner (GP) offices in Maastricht and the surrounding area. IBS patients were subtyped based on predominant bowel habits according to the Rome III criteria. IBS diagnosis was confirmed by a gastroenterologist or GP and when indicated endoscopy with biopsies, abdominal imaging by ultrasonography or CT scan, and/or blood, breath and faecal analysis were performed to exclude organic disease. Patients with a history of abdominal surgery, apart from appendectomy, laparoscopic cholecystectomy and hysterectomy were excluded. 
Healthy controls ( $\mathrm{HC}$ ) between 18-75 years of age were recruited by advertisements in newspapers, on bulletin boards at Maastricht University and on a website for subject recruitment. A brief medical history was taken to rule out history of gastrointestinal disorders or presence of gastrointestinal complaints.

Use of medication was recorder for all subjects.

\section{Assessment of plasma 5-HT and 5-HIAA}

Participants arrived at the MUMC+ after an overnight fast. Blood samples were drawn from the antecubital vein using precooled K2EDTA tubes. To avoid oxidative breakdown of platelets, $0,1 \mathrm{ml} \mathrm{1.4 \%} \mathrm{ascorbic} \mathrm{acid} \mathrm{(Sigma} \mathrm{Aldrich,} \mathrm{St.} \mathrm{Louis,} \mathrm{MO)} \mathrm{was} \mathrm{added} \mathrm{to} \mathrm{the}$ tubes. PPP samples were obtained by centrifuging tubes at $2300 \mathrm{~g}$ at $4^{\circ} \mathrm{C}$ for 10 minutes. Supernatants were aliquoted and frozen immediately at $-80^{\circ} \mathrm{C}$ until further analysis. Concentration of 5-HT and 5-HIAA were determined blind (i.e. on coded samples) using high-performance liquid chromatography (HPLC) as described previously by Hirowatari Y. et al.. ${ }^{29}$ The lower detection limit of $5-\mathrm{HT}$ and 5 -HIAA was $3.3 \mu \mathrm{g} / \mathrm{l}$ and $0.4 \mu \mathrm{g} / \mathrm{l}$, respectively. Data for subjects below the detection limit were based on the average number between 0 and the detection limit for further analyses. For 5-HT and 5-HIAA the intra-assay coefficient of variability (CV) was $<5 \%$ for both parameters, the interassay $\mathrm{CV}$ was $5 \%$ and $7 \%$, respectively.

\section{Questionnaires}

Within a period of 2 weeks prior to collection of blood samples, questionnaires on demographical characteristics, lifestyle factors and the use of medication were completed by all subjects. Symptoms of abdominal discomfort, abdominal pain, bloating, flatulence, nausea and overall GI symptom burden were scored daily using a 14 day symptom diary. ${ }^{30}$

The validated Dutch version (RAND-36) of the Medical Outcomes Study (MOS) 36-item Short Form Health Survey (SF-36) (New England Medical Centre Hospitals, 1991), was used to measure generic health-related QoL. ${ }^{31}$ The SF-36 consists of eight domains with an outcome between 0 and 100, with questions on functional health, well-being and physical and mental health. High scores indicate a high quality of life. From the 8 domains, a physical (PCS) and mental (MCS) composite score can be derived (and used as a general indicator for QoL). ${ }^{32,33}$

Depressive and anxiety symptoms were assessed using the validated Hospital Anxiety and Depression Scale (HADS) and the State-Trait Anxiety Inventory (STAI) version DY. The HADS questionnaire consists of two 7-item scales, for anxiety and for depression, each with a score ranging from 0-21. A mean score $\geq 8$ on the anxiety or depression subscale shows the presence of anxiety or depressive symptoms with the most optimal balance between sensitivity and specificity, ${ }^{34}$ thereby indicating presence of a possible mood disturbance. ${ }^{35}$ The STAI contains 40 questions on a 4-point scale (1-4), clustered 
into two subscales: i.e. State-anxiety, assessing momentary anxiety symptoms, and Trait-anxiety, measuring how anxious participants 'generally' feel. The questionnaires were completed at home and therefore the State-score was not used in the current analysis, as it represents momentary anxiety which did not match with the time of blood sampling. A score of 20-39 indicates low, 40-59 moderate and 60-80 high anxiety in general. $^{36}$

\section{Statistical analysis}

Statistical analysis was performed using IBM SPSS Statistics version 21.0 (IBM Statistics for Windows, Chicago, IL, USA).

Differences in baseline characteristics between IBS patients and HC were tested with the Chi-square test (categorical data), and the independent-samples t-test (for parametric continuous data). Differences between subtypes of IBS and HC were tested using one-way-analysis of variance (ANOVA). To correct for multiple comparisons, the Bonferroni correction was applied.

Linear regression analysis was used to assess the association between the presence of IBS or IBS subtypes and the plasma parameters of serotonin, 5-HIAA and the ratio and to adjust for possible confounding factors. Therefore, we first conducted a crude analysis. Secondly, the statistically significant results were adjusted for possible confounding factors using the backward elimination methods. The following parameters were included in the analyses: age, sex, BMI, psychological (anxiety or depressive) symptoms, current smoking and use of selective serotonin reuptake inhibitors (SSRI), proton pump inhibitors (PPI), and medication that affects motility (i.e. laxatives, prokinetics, opioids, spasmolytics and anti-diarrhoeal drugs). All analyses were performed for IBS patients as a whole group as well as for the subtypes in separate analyses as dummy variables. Missing values were excluded listwise. Data are expressed as mean \pm SD. A two-sided $p$-value $<0.05$ was considered to be statistically significant.

\section{Results}

\section{Baseline characteristics}

From the Maastricht-IBS cohort, we included of subgroup of 154 IBS patients (IBS-Total) and 137 healthy controls (HC) with PPP samples available. With respect to predominant bowel habits, 52 (34\%) were classified as IBS-D, 33 (22\%) as IBS-C, 60 (38\%) as IBS-M and $9(6 \%)$ patients as IBS-U.

Patient characteristics, current medication use, scores for quality of life and anxiety and depression are shown in Table 7.1. No significant differences in age, gender, BMI or alcohol use were observed between IBS and HC. A significant higher percentage of IBS 
patients was smoking at time of inclusion in the cohort $(p<0.01)$. Use of medication also differed significantly: PPIs, SSRIs and both motility decreasing and increasing drugs were significantly more frequently used by IBS patients versus $\mathrm{HC}$ (all $p<0.01$ ). Quality of life (QoL) for both physical and mental scale (SF-PCS and SF-MCS) was significantly impaired in IBS compared to $\mathrm{HC}(\mathrm{p}<0.01)$. Not only were the levels of anxiety and depression symptoms significantly higher in IBS versus HC, but the percentages of IBS patients affected with anxiety and/or depression based on HADS (scores $\geq 8$ ) were also increased in IBS versus HC $(p<0.01)$. Furthermore, Trait-anxiety scores were significantly higher in IBS compared to $\mathrm{HC}(\mathrm{p}<0.01)$. All diary symptom scores were significantly higher in IBS compared to HC (supplementary data, all $\mathrm{p}<0.01$ ). All $p$-values represent significance after correction for multiple testing.

Table 7.1 Baseline characteristics in IBS patients (IBS-Total) and healthy controls (HC).

\begin{tabular}{|c|c|c|c|}
\hline Parameter & $\begin{array}{c}\text { IBS-Total } \\
n=154\end{array}$ & $\begin{array}{c}\mathrm{HC} \\
n=137\end{array}$ & \\
\hline \multicolumn{4}{|l|}{ Demographics } \\
\hline $\operatorname{Sex}(M / F)$ & $30 \% / 70 \%$ & $39 \% / 61 \%$ & \\
\hline Age (years) & $44.5 \pm 16.3$ & $44.2 \pm 19.3$ & \\
\hline BMI $\left(\mathrm{kg} / \mathrm{m}^{2}\right)$ & $24.9 \pm 4.4$ & $24.0 \pm 3.9$ & \\
\hline Current smokers & $25 \%$ & $8.5 \%$ & $* *$ \\
\hline Alcohol use (\%yes) & $52 \%$ & $51 \%$ & \\
\hline \multicolumn{4}{|l|}{ Actual medication use } \\
\hline PPI & $26 \%$ & $2 \%$ & $* *$ \\
\hline NSAID & $11 \%$ & $9 \%$ & \\
\hline SSRI & $17 \%$ & $2 \%$ & $* *$ \\
\hline Motility - & $30 \%$ & $0 \%$ & $* *$ \\
\hline Motility + & $20 \%$ & $0 \%$ & $* *$ \\
\hline \multicolumn{4}{|l|}{ Quality of life } \\
\hline SF-PCS & $40.9 \pm 10.0$ & $54.6 \pm 5.5$ & $* *$ \\
\hline SF-MCS & $46.3 \pm 11.3$ & $54.1 \pm 7.6$ & $* *$ \\
\hline \multicolumn{4}{|l|}{ Anxiety and depression } \\
\hline HADS-A score & $6.9 \pm 3.8$ & $3.7 \pm 2.8$ & $* *$ \\
\hline HADS-D score & $4.5 \pm 4.0$ & $1.9 \pm 2.6$ & $* *$ \\
\hline HADS-A $\geq 8$ & $34 \%$ & $12 \%$ & $* *$ \\
\hline HADS-D $\geq 8$ & $22 \%$ & $5 \%$ & $* *$ \\
\hline HADS-A and/or $-D \geq 8$ & $38 \%$ & $15 \%$ & $* *$ \\
\hline Anxiety TRAIT score & $40.4 \pm 12.4$ & $30.8 \pm 8.7$ & $* *$ \\
\hline
\end{tabular}

Continuous data expressed as mean \pm SD. Motility $+:$ medication that positively affect motility, i.e. laxatives and prokinetic drugs; Motility - : medication that negatively affect motility, i.e. opioids, spasmolytics and antidiarrhoeal drugs. PCS: physical composite score; MCS: mental composite score. ${ }^{* * *} \mathrm{p}<0.01$ IBS vs. HC after correction for multiple testing

\section{IBS subtype analysis}

Because the number of patients with IBS-U was small, this subgroup was not included in the subtype analyses. No differences were found with respect to age, gender, BMI and use of SSRI, PPI and motility decreasing medication (i.e. anti-diarrheal drugs, 
opioids and spasmolytics) between subtypes (data not shown). Motility increasing medication (i.e. prokinetics and laxatives) did differ significantly between IBS-D (12.0\%), IBS-C (36.7\%) and IBS-M (16.4\%) subtypes ( $p=0.02)$. All diary symptom scores were significantly higher for all subtypes compared to $\mathrm{HC}$ (all $\mathrm{p}<0.001$ ), but no significant differences could be detected between the IBS subtypes apart from diarrhea symptoms in IBS-D and constipation symptoms in IBS-C (Supplementary table S7.1). In Table 7.2, the scores for anxiety, depression and quality of life are presented per IBS subtype. The QoL scores were significantly decreased in all IBS subtypes compared to HC, both for the physical and mental domain (all $p<0.001$ ). Between IBS subgroups no significant differences in QoL were found. The HADS anxiety, depression and Trait-anxiety scores were significantly increased in all subtypes compared to HC. However, a significant higher percentage of affected patients (HADS scores $\geq 8$ ) were only found among IBS-D and IBS-M patients but not among IBS-C patients compared to $\mathrm{HC}(\mathrm{p}<0.01)$.

Table 7.2 QoL, anxiety and depression according to IBS subtype

\begin{tabular}{lccccc}
\hline Parameter & $\mathrm{HC}$ & $\mathrm{IBS}-\mathrm{D}$ & $\mathrm{IBS}-\mathrm{C}$ & IBS-M & IBS-U \\
& $\mathrm{n}=137$ & $\mathrm{n}=52$ & $\mathrm{n}=33$ & $\mathrm{n}=60$ & $\mathrm{n}=9$ \\
\hline SF-PCS & $54.6 \pm 5.5$ & $39.9 \pm 9.7^{* * *}$ & $38.0 \pm 10.7^{* * *}$ & $42.7 \pm 9.9 * * *$ & $44.6 \pm 7.8$ \\
SF-MCS & $54.1 \pm 7.6$ & $47.2 \pm 10.8^{* * *}$ & $45.9 \pm 10.4^{* * *}$ & $45.6 \pm 11.9 * * *$ & $46.8 \pm 14.1$ \\
HADS-A score & $3.7 \pm 2.8$ & $7.0 \pm 3.9 * * *$ & $7.0 \pm 3.9 * * *$ & $7.0 \pm 3.8^{* * *}$ & $5.7 \pm 3.5$ \\
HADS-D score & $1.9 \pm 2.6$ & $4.6 \pm 3.6 * * *$ & $4.9 \pm 3.8 * * *$ & $4.4 \pm 4.2 * * *$ & $2.1 \pm 5.3$ \\
HADS-A $\geq 8$ & $12 \%$ & $40 \% * *$ & $27 \%$ & $35 \% * *$ & $11 \%$ \\
HADS-D $\geq 8$ & $5 \%$ & $23 \% * *$ & $17 \%$ & $22 \% * *$ & $22 \%$ \\
HADS-A and/or -D $\geq 8$ & $15 \%$ & $44 \% * *$ & $30 \%$ & $38 \% * *$ & $22 \%$ \\
Anxiety TRAIT score & $30.8 \pm 8.7$ & $40.8 \pm 13.4^{* * *}$ & $40.4 \pm 10.0 * * *$ & $40.5 \pm 12.1^{* * *}$ & $38.0 \pm 16.1$ \\
\hline
\end{tabular}

Continuous data are expressed as mean $\pm S D ;{ }^{*} p<0.01 ; * * p<0.001$ Subtype vs. HC. One-way ANOVA with Bonferroni correction was used for continuous data and $\mathrm{Chi}^{2}$, corrected for multiple testing by multiplying $\mathrm{p}$ value $\times 4$ for dichotomous data. No significant differences could be detected between IBS subtypes for all variables.

\section{Plasma 5-HT, 5-HIAA and 5-HIAA/5HT ratio}

In total, $42 \%$ of IBS patients and $42 \%$ of controls had $5-\mathrm{HT}$ concentrations below the detection limit of $3.3 \mu \mathrm{g} / \mathrm{l}$. Data for 5 -HIAA were in the detectable range for all subjects. The data of the crude linear regression analyses of the IBS-Total, IBS subgroups and HC are presented in Table 7.3A.

The baseline 5-HT levels (Figure 7.1) did not differ significantly between IBS and HC $(7.2 \pm 14.9$ versus $7.1 \pm 8.8 \mathrm{ug} / \mathrm{l})$. However, 5-HIAA concentrations (Figure 7.2) were significantly lower in IBS compared to controls: $24.6 \pm 21.9$ versus $39.0 \pm 29.5 \mathrm{ug} / \mathrm{l}$, respectively $(p<0.001)$.

After expanding the model with confounding variables (Table 7.3B), 5-HIAA levels remained significantly lower in IBS-Total compared to $\mathrm{HC}(\beta-11.6,95 \% \mathrm{Cl}$ : $-19.8 ;-3.4$, $\mathrm{p}=0.006$ ). Backward elimination did not reveal a significant influencing parameter in this model but did show a trend for gender $(p=0.06)$. Subtype analysis for 5-HIAA showed all subtypes to be significantly different compared to HC (Table 7.3A, Figure 
7.2). For the subtype analyses we also adjusted the results for confounding factors. While for IBS-M and IBS-C subtypes 5-HIAA levels remained significantly lower compared to HC, IBS-D versus HC was no longer statistically significant (Table 7.3B).

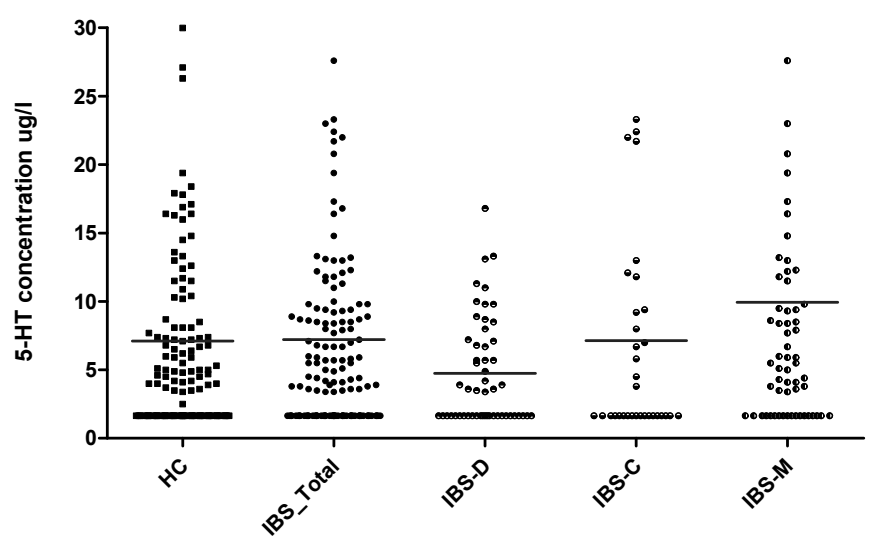

Figure 7.1 Scatterplot for 5-HT concentrations, presented for healthy controls (HC), the total IBS group (IBS-Total) and three subtypes: IBS-D, IBS-C, and IBS-M. IBS-U was not presented due a to small sample size. There were no statistically significant differences between groups. The following dots with a value $>30 \mu \mathrm{g} / \mathrm{I}$ were left out of the graph to improve the visibility of the data, but were included in the analyses: HC 36.6; 43.5; 46.1; 53.9, IBS-C 32.5, IBS-M $175.8 \mathrm{ug} / \mathrm{l}$.

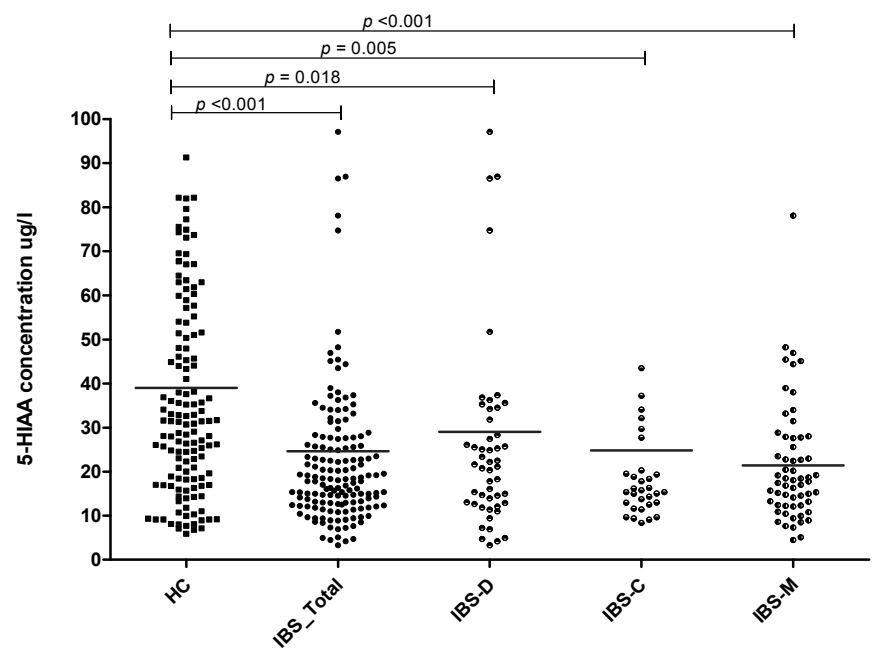

Figure 7.2 Scatterplot for 5-HIAA concentrations, presented for HC, IBS-Total, and three subtypes: IBS-D, IBS-C, and IBS-M. IBS-U was not presented due a to small sample size. Significant differences presented in the graph. The following dots with a value $>100 \mu \mathrm{g} / \mathrm{l}$ were left out of the graph to improve the visibility of the data, but were included in the analyses: HC 104.9; $125.1 ; 125.5$; 127.6; 211.9, IBS-D 103.3; 117.3, IBS-C 115.3; $140.0 \mathrm{ug} / \mathrm{l}$. 


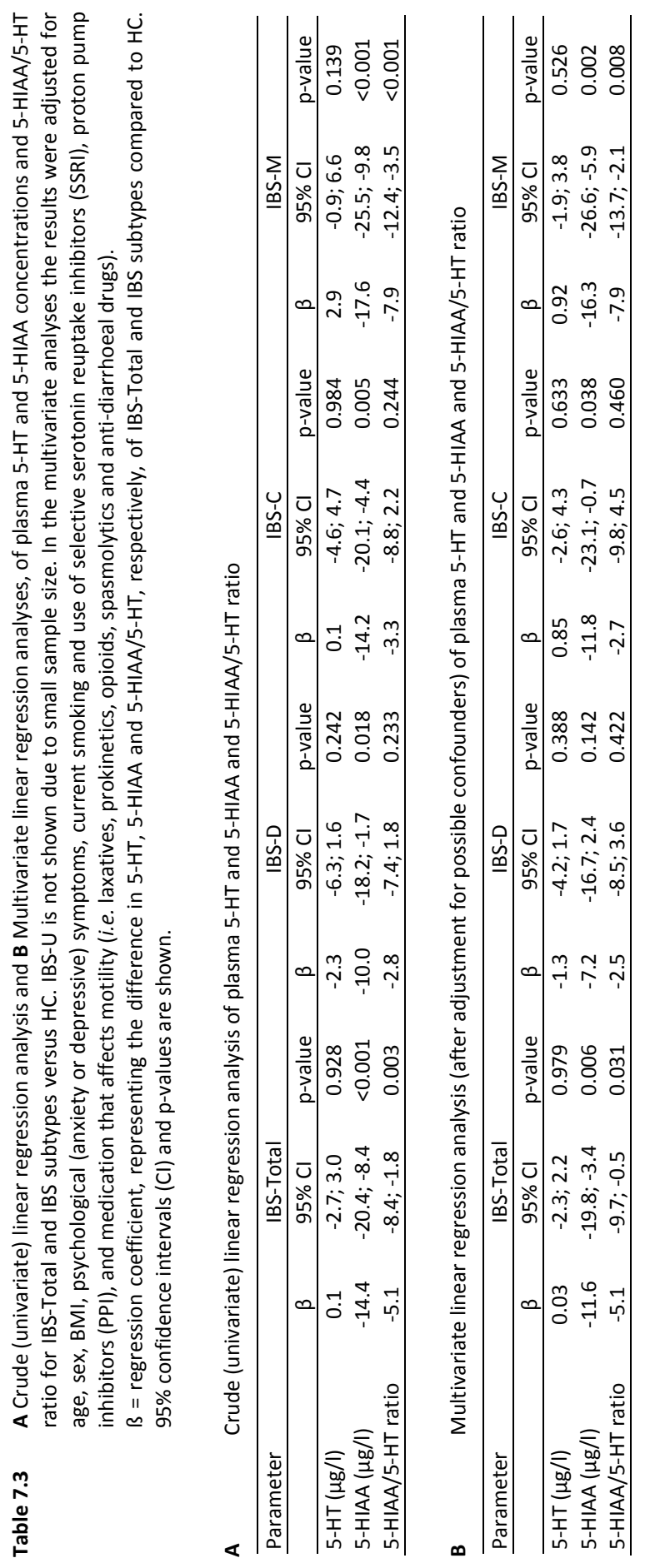


The 5-HIAA/5-HT ratio (Table 7.3A, Figure 7.3) was significantly lower in IBS compared to controls: $(8.4 \pm 12.2$ versus $13.5 \pm 16.6$, respectively $\mathrm{p}<0.01)$ and in IBS-M compared to controls $(5.5 \pm 5.6$ versus $13.5 \pm 16.6$, respectively, $p<0.001)$. Furthermore, the $5-\mathrm{HIAA} / 5-\mathrm{HT}$ ratio was significantly lower in IBS-M versus IBS-D $(\beta-5.1,95 \% \mathrm{Cl}$ : -9.1 ; $-1.2 p=0.012$ ). No significant effects were observed for the potential confounding factors tested (Table 7.3B), and no significant differences were found between other IBS-subtypes apart from IBS-M and controls.

Since the linear regression model showed a possible influence of gender on 5-HIAA concentrations, we performed a separate analysis only within the IBS patients group to assess the effect of gender on 5-HIAA concentrations in IBS patients.

Linear regression analysis showed a $\beta$ of 8.1 ( $95 \% \mathrm{Cl}$ : $0.6 ; 15.7, p=0.04$ ), pointing to a significantly lower 5-HIAA concentration in female IBS patients compared to male IBS patients. No influence was found for other patient characteristics tested. To delineate whether the finding was IBS-specific, we repeated this analysis in HC (NS), pointing to an IBS-specific difference in systemic 5-HIAA between females and males.

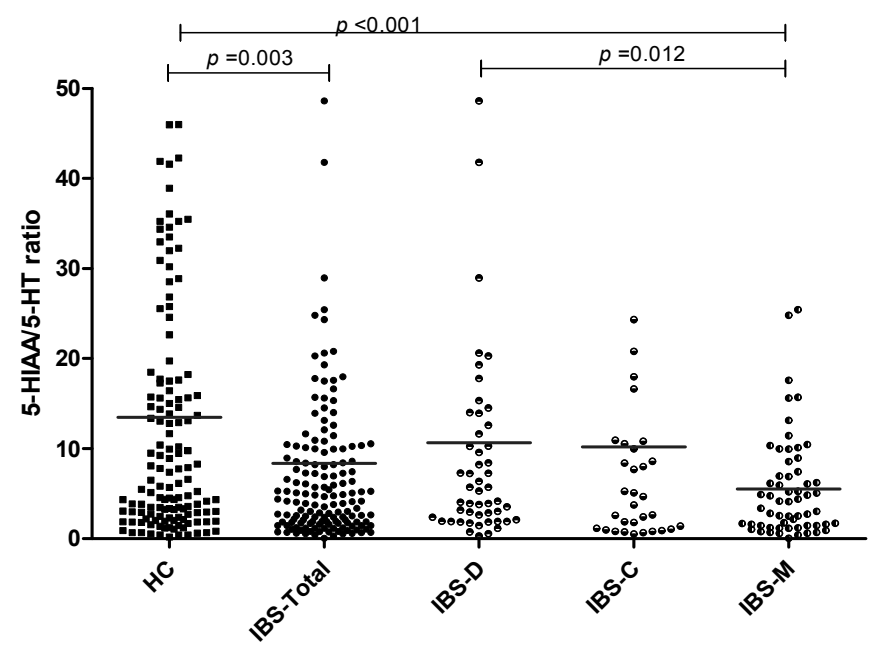

Figure 7.3 Scatterplot for 5-HIAA / 5-HT ratio presented for: HC, IBS-Total, and three subtypes: IBS-D, IBS$C$, and IBS-M. Significant differences presented in the graph.

\section{Discussion}

In a large group of well-characterized IBS patients, no alterations were observed in plasma fasting 5-HT concentrations compared to controls. However, a significant 
decrease in 5-HIAA concentrations and in the 5-HIAA/5-HT ratio was found in patients with IBS. All IBS subtypes showed lower 5-HIAA levels compared to controls, but the difference was most pronounced in the IBS-M group. The 5-HIAA/5-HT ratio also was significantly lower in the total IBS group and IBS-M compared to controls. Apart from a possible gender effect on 5-HIAA levels, no influence was found for abdominal symptoms, anxiety and/or depression nor for medication use on plasma 5-HT, 5-HIAA or their ratio in IBS patients or subgroups.

The fasting 5-HT levels did not significantly differ between IBS or subgroups and HC. Our data confirm previous studies by among others Kilkens et al. ${ }^{22}$ and Houghton et al., ${ }^{19}$ showing that fasting platelet-poor-plasma 5-HT levels in IBS were not different from controls. ${ }^{20}$ In contrast, higher fasting 5-HT levels have been observed by others in IBS-D patients, ${ }^{8,37}$ but also in IBS irrespective of subtype. ${ }^{18}$ Numerous stimuli lead to 5$\mathrm{HT}$ release; i.e. ingestion of food, vagal or sympathetic adrenergic stimulation, lowering of luminal $\mathrm{pH}$, mucosal distortion and obstruction of gut motility. ${ }^{38}$ Several trials that assessed 5-HT levels after meal stimulation found an increase in 5-HT levels in IBS compared to controls. ${ }^{18-20,37}$ Unfortunately, most of these studies did not take into account existing differences in baseline values. ${ }^{18-20}$ Hougthon et al did correct for baseline levels, after which differences in postprandial 5-HT levels were no longer significant. ${ }^{37}$ Atkinson et al. ${ }^{8}$ have shown an increase in $5-\mathrm{HT}$ after meal stimulation in controls and IBS-D but not in IBS-C. In our study, $42 \%$ of the fasting samples had 5-HT concentrations below the detection limit. These data indicate that measurement of systemic 5-HT levels has little relevance for discrimination of IBS from controls and in the future attention should focus (more) on mucosal levels of 5-HT and its metabolites.

We also assessed 5-HIAA, the principal metabolite of 5-HT in the human body, and our data point to a pronounced decrease in 5-HIAA concentrations in IBS patients and in the IBS-D, IBS-C and IBS-M subtypes compared to controls. Previously, a decrease in 5HIAA has been observed in IBS-C patients ${ }^{8}$ but most studies focused on 5-HT and/or ratio data. ${ }^{19-21}$ The lower 5-HIAA concentrations found in our IBS patients may result from a decrease in serotonergic metabolism by a reduced MAO activity (intestinal or extra-intestinal) or decreased uptake of 5-HT by SERT. MAO activity in the brain as well as in peripheral organs has been reported to be reduced in smokers compared to nonsmokers. $^{39,40}$ In our study population, significantly more IBS patients use tobacco compared to the healthy controls. This may partially explain the differences in plasma 5-HIAA concentrations between groups. However, after adjusting the analyses for smoking in a multivariate linear regression model, and also after exclusion of all smokers in a post hoc analyses, the difference between IBS patients and $\mathrm{HC}$ with regard to 5-HIAA concentration remained significant. This indicates that the imbalance in number of smokers between groups does not significantly affect the outcomes with regard to 5-HIAA in our study. 
Previously, mucosal SERT expression was found to be decreased in IBS-C and IBS-D patients, but studies did not report on IBS-M subtype. ${ }^{41-43}$ Decreased mucosal SERT activity in IBS may result in an increase in mucosal availability of 5-HT leading to luminal hypersensitivity, hypermotility and diarrhea. It has been speculated that continuous exposure of the mucosa to 5-HT may eventually result in the desensitization of 5-HT receptors with decreased reflex activity and give rise to luminal hyposecretion and constipation. ${ }^{44,45}$ The role of SERT polymorphisms in enteric serotonergic signaling in humans remains unclear. ${ }^{46}$ Variations in SERT activity and/or expression might lead to changes in enteric serotonergic signaling and hence promote alterations of bowel sensory, secretory and motor functions that could account for abdominal symptoms in $\mathrm{IBS}^{46} \mathrm{~A}$ potential role of SERT activity can be substantiated by evaluating the influence of SSRI use on 5-HT or 5-HIAA levels. ${ }^{11}$ The effects of selective serotonin reuptake inhibitors (SSRIS) are almost entirely due to SERT inhibition, ${ }^{11}$ resulting in increased serotonergic activity. Previous studies exploring the influence of SSRI use on 5-HT metabolism have resulted in contradicting results with respect to 5-HT cerebrospinal fluid concentrations before and after acute SSRI administration. ${ }^{47,48}$ Acute administration of citalopram in IBS patients resulted in increased PPP 5-HT levels in combination with enhanced memory performance, leading to the assumption that SSRI administration increases 5-HT at synapses, but it is still uncertain how this may affect 5-HT activity at CNS or intestinal level. ${ }^{22}$ We anticipated to find differences in 5-HT metabolism markers with regard to SSRI use, but our data did not reveal any influence. Until now, little is known about the chronic use of SSRIs on 5-HT synthesis. ${ }^{49}$ Our data do not provide evidence for an influence of chronic SSRI use on systemic 5-HT and 5-HIAA concentrations. Possibly, the effect of SSRIs on plasma levels of 5-HT and 5-HIAA are concealed by transporters which function as 'back up' for uptake of serotonin into the cells, i,e. organic cation and dopamine transporters, when SERT is inhibited. We have not assessed SERT activity, which is a limitation of the present study. However, future focus should be directed more towards local, i.e. intestinal, instead of systemic level serotonin metabolism.

Anxiety and depression are more prevalent among IBS patients compared to the general population. ${ }^{30,50}$ In our own tertiary center for referral of patients with complex functional gastrointestinal disorders (FGIDs), in as much as $50 \%$ of the patients an anxiety disorder is diagnosed and in $20 \%$ a mood disorder. ${ }^{51} \mathrm{~A}$ high prevalence of anxiety and depression was confirmed in our IBS cohort, including patients from GPs, secondary and tertiary referral. Changes in serotonin metabolism have been reported to be more pronounced in patients with psychiatric co-morbidity. ${ }^{10,25}$ We did not observe a significant influence of anxiety and/or depression symptoms on plasma markers of 5-HT metabolism. A few other studies have assessed the correlation between plasma 5 -HT metabolism and anxiety/depression symptoms in IBS patients 
but results were not in line with each other and do not point to a specific direction of influence by anxiety or depression disorders on 5 -HT metabolism. ${ }^{18,26}$

With regard to the relation between the use of PPIs and serotonin metabolism, limited data are available. PPI use is reported to be associated with higher levels of chromogranin $A$, which is produced by enterochromaffin cells, alike most of the serotonin that is present in GI tract and blood plasma. However, whether PPI use leads to altered 5-HT or 5-HIAA plasma levels is unknown. Our analyses did not demonstrate a significant association between these markers and the use of PPIs, also after exclusion of all PPI users in a post hoc analyses, the difference between IBS patients and HC with regard to 5-HIAA concentration remained significant.

Furthermore, drugs with enterokinetic activities may also affect and even target serotonin metabolism. Certain prokinetics target the 5-HT4 receptor, e.g. prucalopride ${ }^{52}$ to accelerate gastrointestinal and colonic transit. On the other hand 5-HT3 receptor antagonists, such as ondansetron ${ }^{53}$ and alosetron ${ }^{54}$ have antiemetic properties and slow down the movement of chyme through the GI tract. Both, the 5-HT4 receptor agonists and 5-HT3 receptor antagonists, have been demonstrated to reduce symptom burden in subgroups of IBS patients, ${ }^{55}$ which further substantiates the role of serotonin in GI physiology and possibly also IBS pathophysiology. However, in our study population none of the subjects used 5-HT4 receptor agonists or 5-HT3 receptor antagonists. Furthermore, our data do not show any significant confounding effects of any drugs that affect motility with regard to 5-HT and 5-HIAA plasma levels in IBS patients and $\mathrm{HC}$.

Our study is the first to observe distinctly different results for IBS-M patients with respect to serotonin metabolism with a decreased 5-HIAA/5-HT ratio. The differences in the IBS-M group are not explained by symptom scores, psychological comorbidity, gender or use of medication. In previous studies on serotonin metabolism, this subgroup of IBS patients has not been analyzed separately. ${ }^{8,18-20}$ This is remarkable since the IBS-M subtype is the most common IBS subtype. ${ }^{56-58}$ With regard to $5-\mathrm{HT}$ metabolism in our study, IBS-M seemed not simply a mix of IBS-D and IBS-C data. IBS-M patients had the lowest 5-HIAA levels and 5-HIAA/5-HT ratio compared to all the other subtypes. A recently published large scale study showed significantly higher scores for IBS-M patients regarding somatization and somatic symptom scores compared to either IBS-C or IBS-D. ${ }^{59}$ However, in another study no significant differences between IBS-D, IBS-C and IBS-M with respect to IBS severity, abdominal pain, level of somatization, QoL and anxiety or depression scores were found. ${ }^{60}$ We postulate, based on the differences in particularly 5-HIAA/5-HT ratio, that IBS-M may be a truly separate entity in IBS, possibly with a different pathophysiological background in comparison to IBS-D and IBS-C. 
Linear regression analysis revealed an influence of gender on 5-HIAA concentrations in IBS but not in controls. Female IBS patients had significantly lower 5-HIAA concentrations compared to IBS males. Previously, female patients were found to have lower SERT expression, which could explain lower 5-HIAA concentrations. ${ }^{61}$ Differences in systemic 5-HT between males and females have also been observed previously in one study, in which fasting PPP 5-HT levels were significantly higher compared to HC, which was explained after stratifying the groups by IBS-D men only. A relation with menstrual cycle could not be proven in that study. No gender differences were found in 5-HIAA concentrations. ${ }^{37}$ The lower 5-HIAA levels we observed in IBS females cannot be explained by differences in subtype distribution, psychological comorbidity or use of medication. Menstrual cycle phase was not taken into account in our study, neither were stress factors, which have been shown to be potential confounders. ${ }^{13,26}$

Although we assessed 5-HT and 5-HIAA plasma levels in a relatively large heterogeneous IBS population with sufficient numbers of subtype populations, a study including a larger number of patients in separate IBS subtype groups would be warranted to confirm our findings in the subgroup analyses. Furthermore, it has to be noted that our results are based on cross-sectional and observational data and that causality was not studied.

The method we employed for assessment of 5-HT and 5-HIAA in PPP has previously been described by Hirowatari et al., ${ }^{29}$ and is considered to be a reliable method with adequate specificity, sensitivity and reproducibility. It should be acknowledged that in our study $42 \%$ of IBS patients and comparable numbers of controls had 5-HT concentrations below the detection limit, a finding in line with data by Bearcroft et al.. ${ }^{20}$ Brand et al systematically reviewed 101 trials that have measured 5-HT in PPP and found that methods to measure $5-\mathrm{HT}$ vary widely. ${ }^{62}$ The concentration of $5-\mathrm{HT}$ in platelets may exceed free plasma concentration, leaving the possibility open that what is measured in PPP reflects leakage of $5-\mathrm{HT}$ from platelets during preparation instead of the actual concentration in plasma. ${ }^{11}$ Therefore, the assessment of 5-HIAA levels seems more useful for the assessment of systemic serotonin metabolism than the quantification of 5-HT itself. However, due to the wide distribution of 5-HIAA levels in both IBS patients and $\mathrm{HC}$ and the indication that the systemic measurements not fully represents or reflects intestinal metabolism, both 5-HT and 5-HIAA plasma levels do not seem to be very useful as a potential biomarker in IBS patients. Furthermore, this is a rather crude way to evaluate the pleiotropic effects and complex metabolism of 5-HT in the gut physiology. Assessment of serotonin metabolism at intestinal level, combined with systemic measurements, of 5-HT and 5-HIAA may help to provide a better insight into the serotonin metabolismin IBS, ${ }^{8,27}$ however, a wide spread in mucosal assessment of $5-\mathrm{HT}$ has also been seen in previously performed studies. ${ }^{63}$ 


\section{Conclusion}

Fasting plasma 5-HT levels were not significantly different between IBS patients and healthy controls. However, our data showed a significant decrease in fasting 5-HIAA concentrations and 5-HIAA/5-HT ratio in IBS, in which all IBS subtypes separately have lower 5-HIAA levels compared to controls and the 5-HIAA/5-HT ratio was significantly lower in IBS-M versus controls. Linear regression analysis points to an influence of gender but not of symptoms, anxiety and/or depression or medication use.

Our study demonstrated that although fasting 5-HT plasma levels are not changed in IBS compared to controls, the differences in 5-HIAA levels may reflect altered serotonin metabolism in IBS patients. 


\section{References}

1 Longstreth GF, Thompson WG, Chey WD, Houghton LA, Mearin F, Spiller RC. Functional bowel disorders. Gastroenterology 2006;130:1480-91.

2 Hungin AP, Whorwell PJ, Tack J, Mearin F. The prevalence, patterns and impact of irritable bowel syndrome: an international survey of 40,000 subjects. Aliment Pharmacol Ther 2003; 17:643-50.

3 Olden KW. Diagnosis of irritable bowel syndrome. Gastroenterology 2002;122:1701-14.

4 Camilleri M, Heading RC, Thompson WG. Clinical perspectives, mechanisms, diagnosis and management of irritable bowel syndrome. Aliment Pharmacol Ther 2002;16:1407-30.

5 El-Salhy M. Irritable bowel syndrome: diagnosis and pathogenesis. World J Gastroenterol 2012;18: 5151-63.

6 Drossman DA, Camilleri M, Mayer EA, Whitehead WE. AGA technical review on irritable bowel syndrome. Gastroenterology 2002;123:2108-31.

7 Kim DY, Camilleri M. Serotonin: a mediator of the brain-gut connection. Am J Gastroenterol 2000;95: 2698-709.

8 Atkinson W, Lockhart S, Whorwell PJ, Keevil B, Houghton LA. Altered 5-hydroxytryptamine signaling in patients with constipation- and diarrhea-predominant irritable bowel syndrome. Gastroenterology 2006;130:34-43.

9 Berger M, Gray JA, Roth BL. The expanded biology of serotonin. Ann Rev Med 2009;60:355-66.

10 Cremon C, Carini G, Wang B, Vasina V, Cogliandro RF, De Giorgio R, Stanghellini V, Grundy D, Tonini M, De Ponti F, Corinaldesi R, Barbara G. Intestinal serotonin release, sensory neuron activation, and abdominal pain in irritable bowel syndrome. Am J Gastroenterol 2011;106:1290-8.

11 Gershon MD, Tack J. The serotonin signaling system: from basic understanding to drug development for functional GI disorders. Gastroenterology 2007;132:397-414.

12 Gershon MD. Review article: serotonin receptors and transporters -- roles in normal and abnormal gastrointestinal motility. Aliment Pharmacol Ther 2004;20 Suppl 7:3-14.

13 Matricon J, Meleine M, Gelot A, Piche T, Dapoigny M, Muller E, Ardid D. Review article: Associations between immune activation, intestinal permeability and the irritable bowel syndrome. Aliment Pharmacol Ther 2012;36:1009-31.

14 Mawe GM, Coates MD, Moses PL. Review article: intestinal serotonin signalling in irritable bowel syndrome. Aliment Pharmacol Ther 2006;23:1067-76.

15 Tack J, Janssen P, Wouters M, Boeckxstaens G. Targeting serotonin synthesis to treat irritable bowel syndrome. Gastroenterology 2011;141:420-2.

16 Erspamer V, Testini A. Observations on the release and turnover rate of 5-hydroxytryptamine in the gastrointestinal tract. J Pharm Pharmacol 1959;11:618-23.

17 Nikisch G, Mathe AA, Czernik A, Eap CB, Jimenez-Vasquez P, Brawand-Amey M, Baumann $P$. Stereoselective metabolism of citalopram in plasma and cerebrospinal fluid of depressive patients: relationship with 5-HIAA in CSF and clinical response. J Clin Psychopharmacol 2004;24:283-90.

18 Park SY, Park MH, Yoon KW, Cho SB, Lee WS, Park CH, Kim HS, Choi SK, Rew JS. Plasma 5hydroxytryptamine concentration and its correlation with psychopathology in patients with irritable bowel syndrome. Gut Liver 2009;3:26-30.

19 Houghton LA, Atkinson W, Whitaker RP, Whorwell PJ, Rimmer MJ. Increased platelet depleted plasma 5-hydroxytryptamine concentration following meal ingestion in symptomatic female subjects with diarrhoea predominant irritable bowel syndrome. Gut 2003;52:663-70.

20 Bearcroft CP, Perrett D, Farthing MJ. Postprandial plasma 5-hydroxytryptamine in diarrhoea predominant irritable bowel syndrome: a pilot study. Gut 1998;42:42-6.

21 Dunlop SP, Coleman NS, Blackshaw E, Perkins AC, Singh G, Marsden CA, Spiller RC. Abnormalities of 5hydroxytryptamine metabolism in irritable bowel syndrome. Clin Gastroenterol Hepatol 2005;3:349-57.

22 Kilkens TO, Honig A, Fekkes D, Brummer RJ. The effects of an acute serotonergic challenge on brain-gut responses in irritable bowel syndrome patients and controls. Aliment Pharmacol Ther 2005;22:865-74.

23 Bradford K, Shih W, Videlock EJ, Presson AP, Naliboff BD, Mayer EA, Chang L. Association between early adverse life events and irritable bowel syndrome. Clin Gastroenterol Hepatol 2012;10:385-90 e381-3. 
24 Sykes MA, Blanchard EB, Lackner J, Keefer L, Krasner S. Psychopathology in irritable bowel syndrome: support for a psychophysiological model. J Behav Med 2003;26:361-72.

25 Kim JJ. Serotonin, stress, and conditioning. Biol Psychiatry 2008;63:819-20.

26 Stasi C, Bellini M, Costa F, Mumolo MG, Ricchiuti A, Grosso M, Duranti E, Metelli MR, Gambaccini D, Bianchi L, Di Tanna GL, Laffi G, Taddei S, Marchi S. Neuroendocrine markers and psychological features in patients with irritable bowel syndrome. Int J Colorectal Dis 2013;28:1203-8.

27 Keszthelyi D, Troost FJ, Jonkers DM, Kruimel JW, Leue C, Masclee AA. Decreased levels of kynurenic acid in the intestinal mucosa of IBS patients: relation to serotonin and psychological state. J Psychosom Res 2013;74:501-4.

28 Faresjo A, Grodzinsky E, Johansson S, Wallander MA, Faresjo T, Timpka T. Self-reported use of pharmaceuticals among patients with irritable bowel syndrome in primary care. J Manag Care Pharm 2008;14:870-7.

29 Hirowatari Y, Hara K, Kamihata H, Iwasaka T, Takahashi H. High-performance liquid chromatographic method with column-switching and post-column reaction for determination of serotonin levels in platelet-poor plasma. Clin Biochem 2004;37:191-7.

30 Thijssen AY, Jonkers DM, Leue C, van der Veek PP, Vidakovic-Vukic M, van Rood YR, Clemens $\mathrm{CH}$, Masclee AA. Dysfunctional cognitions, anxiety and depression in irritable bowel syndrome. J Clin Gastroenterol 2010;44:e236-41.

31 van der Zee KI SR. Het meten van de algemene gezonsheidstoestand met de RAND-36, een handleiding. [Assessment of general health status with the RAND-36; guidebook]. Noordelijk Centrum voor Gezondheidsvraagstukken Rijksuniversiteit Groningen: Groningen, 1993

32 Creed F, Ratcliffe J, Fernandez L, Tomenson B, Palmer S, Rigby C, Guthrie E, Read N, Thompson D. Health-related quality of life and health care costs in severe, refractory irritable bowel syndrome. Ann Int Med 2001;134:860-8.

33 Farivar SS, Cunningham WE, Hays RD. Correlated physical and mental health summary scores for the SF-36 and SF-12 Health Survey, V.I. Health Qual Life Outcomes 2007;5:54.

34 Bjelland I, Dahl AA, Haug TT, Neckelmann D. The validity of the Hospital Anxiety and Depression Scale. An updated literature review. J Psychosom Res 2002;52:69-77.

35 Zigmond AS, Snaith RP. The hospital anxiety and depression scale. Acta Psychiatr Scand 1983;67: 361-70.

36 Spielberger GaL. State-trait Anxiety Inventory. Consulting Psychologists Press 1970

37 Houghton LA, Brown H, Atkinson W, Morris J, Fell C, Whorwell PJ, Lockhart S, Keevil B. 5hydroxytryptamine signalling in irritable bowel syndrome with diarrhoea: effects of gender and menstrual status. Aliment Pharmacol Ther 2009;30:919-29.

38 McLean PG, Borman RA, Lee K. 5-HT in the enteric nervous system: gut function and neuropharmacology. Trends Neurosci 2007;30:9-13.

39 Fowler JS, Logan J, Wang GJ, Volkow ND, Telang F, Zhu W, Franceschi D, Pappas N, Ferrieri R, Shea C, Garza V, Xu Y, Schlyer D, Gatley SJ, Ding YS, Alexoff D, Warner D, Netusil N, Carter P, Jayne M, King P, Vaska P. Low monoamine oxidase B in peripheral organs in smokers. Proc Natl Acad Sci U S A 2003;100: 11600-5.

40 Fowler JS, Volkow ND, Wang GJ, Pappas N, Logan J, Shea C, Alexoff D, MacGregor RR, Schlyer DJ, Zezulkova I, Wolf AP. Brain monoamine oxidase A inhibition in cigarette smokers. Proc Natl Acad Sci U S A 1996;93:14065-9.

41 Foley S, Garsed K, Singh G, Duroudier NP, Swan C, Hall IP, Zaitoun A, Bennett A, Marsden C, Holmes G, Walls A, Spiller RC. Impaired uptake of serotonin by platelets from patients with irritable bowel syndrome correlates with duodenal immune activation. Gastroenterology 2011;140:1434-43 e1431.

42 Kerckhoffs AP, ter Linde JJ, Akkermans LM, Samsom M. SERT and TPH-1 mRNA expression are reduced in irritable bowel syndrome patients regardless of visceral sensitivity state in large intestine. Am J Physiol Gastrointest Liver Physiol 2012;302:G1053-60.

43 El-Salhy M, Wendelbo I, Gundersen D. Serotonin and serotonin transporter in the rectum of patients with irritable bowel disease. Mol Med Rep 2013;8:451-5. 
44 Coates MD, Mahoney CR, Linden DR, Sampson JE, Chen J, Blaszyk H, Crowell MD, Sharkey KA, Gershon MD, Mawe GM, Moses PL. Molecular defects in mucosal serotonin content and decreased serotonin reuptake transporter in ulcerative colitis and irritable bowel syndrome. Gastroenterology 2004;126: 1657-64.

45 Shekhar C, Monaghan PJ, Morris J, Issa B, Whorwell PJ, Keevil B, Houghton LA. Rome III functional constipation and irritable bowel syndrome with constipation are similar disorders within a spectrum of sensitization, regulated by serotonin. Gastroenterology 2013;145:749-57; quiz e713-44.

46 Colucci R, Blandizzi C, Bellini M, Ghisu N, Tonini M, Del Tacca M. The genetics of the serotonin transporter and irritable bowel syndrome. Trends Mol Med 2008;14:295-304.

47 Carpenter LL, Anderson GM, Siniscalchi JM, Chappell PB, Price LH. Acute changes in cerebrospinal fluid 5-HIAA following oral paroxetine challenge in healthy humans. Neuropsychopharmacology 2003;28: 339-47.

48 Barton DA, Esler MD, Dawood T, Lambert EA, Haikerwal D, Brenchley C, Socratous F, Hastings J, Guo L, Wiesner G, Kaye DM, Bayles R, Schlaich MP, Lambert GW. Elevated brain serotonin turnover in patients with depression: effect of genotype and therapy. Arch Gen Psychiatry 2008;65:38-46.

49 Honig G, Jongsma ME, van der Hart MC, Tecott LH. Chronic citalopram administration causes a sustained suppression of serotonin synthesis in the mouse forebrain. PloS One 2009;4:e6797.

50 Levy RL, Olden KW, Naliboff BD, Bradley LA, Francisconi C, Drossman DA, Creed F. Psychosocial aspects of the functional gastrointestinal disorders. Gastroenterology 2006;130 1447-58.

51 Kruimel J, Leue C, Winkens B, Marcus D, Schoon S, Dellink R, van Os J, Masclee A. Integrated medicalpsychiatric outpatient care in functional gastrointestinal disorders improves outcome: a pilot study. Eur J Gastroenterol Hepatol 2015;27:721-7.

52 Bouras EP, Camilleri M, Burton DD, Thomforde G, McKinzie S, Zinsmeister AR. Prucalopride accelerates gastrointestinal and colonic transit in patients with constipation without a rectal evacuation disorder. Gastroenterology 2001;120:354-60.

53 Cooke CE, Mehra IV. Oral ondansetron for preventing nausea and vomiting. Am J Hosp Pharm 1994;51: 762-71.

54 Gunput MD. Review article: clinical pharmacology of alosetron. Aliment Pharmacol Ther 1999;13 Suppl 2:70-6.

55 Tack J, Fried M, Houghton LA, Spicak J, Fisher G. Systematic review: the efficacy of treatments for irritable bowel syndrome--a European perspective. Aliment Pharmacol Ther 2006;24:183-205.

56 Wilson S, Roberts L, Roalfe A, Bridge P, Singh S. Prevalence of irritable bowel syndrome: a community survey. Br J Gen Pract 2004;54:495-502.

57 Lin S, Mooney PD, Kurien M, Aziz I, Leeds JS, Sanders DS. Prevalence, investigational pathways and diagnostic outcomes in differing irritable bowel syndrome subtypes. Eur J Gastroenterol Hepatol 2014; 26:1176-80.

58 Ford AC, Bercik P, Morgan DG, Bolino C, Pintos-Sanchez MI, Moayyedi P. Characteristics of functional bowel disorder patients: a cross-sectional survey using the Rome III criteria. Aliment Pharmacol Ther 2014;39:312-21.

59 Patel P, Bercik P, Morgan DG, Bolino C, Pintos-Sanchez MI, Moayyedi P, Ford AC. Irritable bowel syndrome is significantly associated with somatisation in 840 patients, which may drive bloating. Aliment Pharmacol Ther 2015;41:449-58.

60 Rey de Castro NG, Miller V, Carruthers HR, Whorwell PJ. Irritable bowel syndrome: a comparison of subtypes. J Gastroenterol Hepatol 2015;30:279-85.

61 Franke L, Schmidtmann M, Riedl A, van der Voort I, Uebelhack R, Monnikes H. Serotonin transporter activity and serotonin concentration in platelets of patients with irritable bowel syndrome: effect of gender. J Gastroenterol 2010;45:389-98.

62 Brand T, Anderson GM. The measurement of platelet-poor plasma serotonin: a systematic review of prior reports and recommendations for improved analysis. Clin Chem 2011;57:1376-86.

63 Keszthelyi D, Troost FJ, Jonkers DM, van Donkelaar EL, Dekker J, Buurman WA, Masclee AA. Does acute tryptophan depletion affect peripheral serotonin metabolism in the intestine? Am J Clin Nutr 2012;95: 603-8. 


\section{Supplementary data}

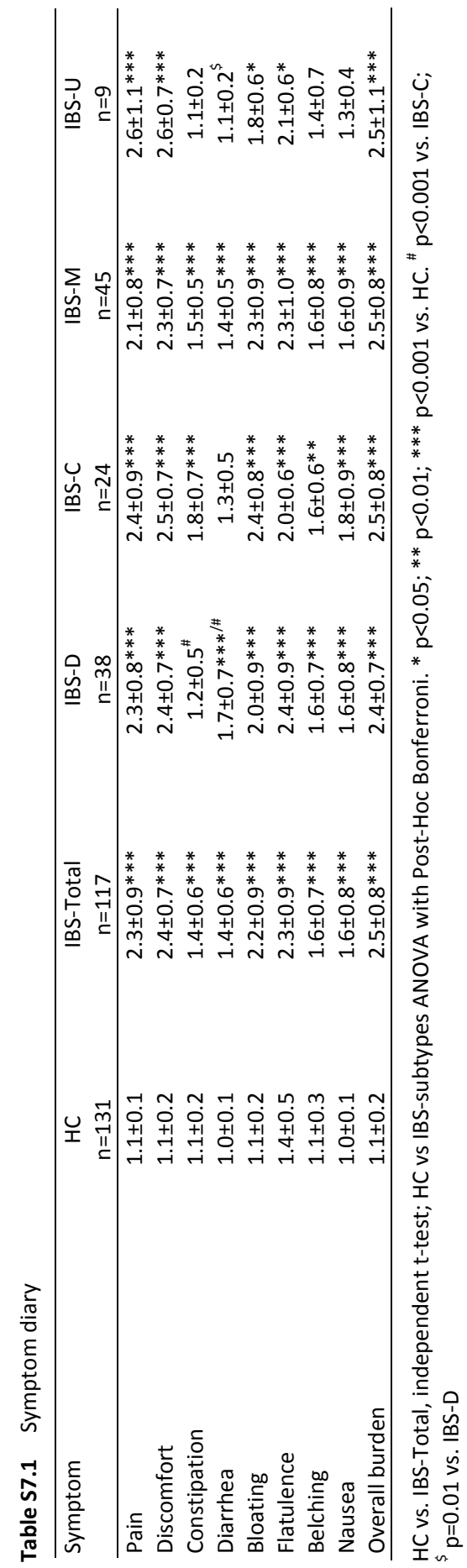


Serotonin metabolism in IBS 


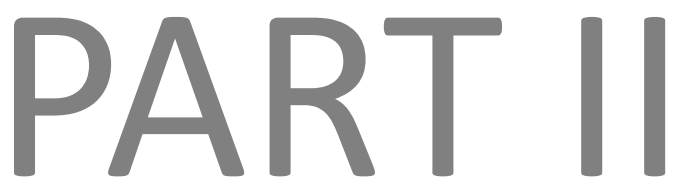

Symptoms 


\section{Chapter 8}

\section{Systematic review:}

\section{Measurement of abdominal pain in IBS}

Systematic review: instruments to assess abdominal pain in irritable bowel syndrome Zlatan Mujagic, Daniel Keszthelyi, Qasim Aziz, Walter Reinisch, Emilio G Quetglas, Francesco De Leonardis, Märta Segerdahl, Ad AM Masclee Alimentary Pharmacology \& Therapeutics 2015;42:1064-1081 


\section{Abstract}

\section{Background}

Consensus on standard methods to assess chronic abdominal pain in patients with irritable bowel syndrome (IBS) is currently lacking.

\section{Aim}

To systematically review the literature with respect to instruments of measurement of chronic abdominal pain in IBS patients.

\section{Methods}

Systematic literature search was performed in PubMed / Medline databases for studies using pain measurement instruments in patients with IBS.

\section{Results}

110 publications were reviewed. A multitude of different instruments is currently used to assess chronic abdominal pain in IBS patients. The single item methods, e.g. the validated 10-point numeric rating scale (NRS), and questionnaires assessing gastrointestinal symptoms severity, focus on the assessment of only the intensity of abdominal pain. Of these questionnaires the validated IBS-Symptom Severity Scale includes the broadest measurement of pain related aspects. General pain questionnaires and electronic momentary symptom assessment tools have been used to study abdominal pain in IBS patients as well, but have not yet been validated for this purpose. The evidence for the use of provocation tests, e.g. the rectal barostat with balloon distention, for measurement of abdominal pain in IBS is weak, due to the poor correlation between visceral pain thresholds assessed by provocation tests and abdominal pain as assessed by questionnaires.

\section{Conclusions}

The multitude of different instruments to measure chronic abdominal pain in IBS leads to difficult to compare endpoints of published studies. There is need for a validated instrument to assess chronic abdominal pain in IBS patients that overcome the limitations of the currently available methods. 


\section{Introduction}

Abdominal pain occurs frequently as a disabling symptom that is experienced by patients in daily clinical care practice (e.g. gastroenterology, gynaecology, surgery, urology). Chronic recurrent abdominal pain is a typical and prominent characteristic of irritable bowel syndrome (IBS). In patients who are affected, this symptom results in a severe burden and impairment of quality of life. ${ }^{1}$ Currently available treatments aimed to relieve abdominal pain, including non-steroidal anti-inflammatory drugs, opioids, tricyclic antidepressants and selective serotonin/noradrenalin reuptake inhibitors, are frequently used but generally not attractive therapeutic entities in IBS, due to targetrelated side effects. Thus, efficacious therapeutic pain strategies are lacking. Development of novel therapies is hindered by an incomplete understanding of mechanisms involved in the pathogenesis of abdominal pain. In addition, a drawback in the development and application of analgesics for treatment of visceral pain in patients with IBS may, in part, be the current lack of reliable instruments to measure and quantify chronic abdominal pain.

The International Association for the Study of Pain (IASP) defines chronic pain as 'persistent pain, which can be either continuous or recurrent, of sufficient duration and intensity to adversely affect a patient's wellbeing, level of function and quality of life.' The duration of chronic pain, both of somatic and visceral origin, is arbitrarily set at three months or longer. ${ }^{3}$ For IBS patients, The Rome III criteria define chronic recurrent abdominal pain, as present at least 3 days a month in the last three months, with the unset at least before 6 months. ${ }^{4}$ In contrast, acute pain is an instant reaction to noxious or potentially noxious stimuli, declines within the time interval necessary for healing and serves an useful temporary physiological purpose, motivating the body to protect the organ while it heals. ${ }^{5}$ Up until fifteen years ago, most information on underlying basic mechanisms of pain had been derived from experimental studies of somatic nociception, the neural processing of noxious stimuli on e.g. skin, muscles or joints. Chronic pain models were generally based on these studies, assuming that the same neurological mechanisms are responsible for all forms of pain. In the past fifteen years it became evident that both mechanisms and presentation of visceral and somatic pain differ at various levels. Visceral sensations may even differ between organs. ${ }^{6,7}$ Chronic abdominal pain is neurobiologically considered to be visceral pain, i.e. pain arising from internal organs. It is manifested in an acute, recurrent or chronic form and is vague, diffuse and poorly defined by patients. The intensity of the visceral pain is poorly related to the magnitude of the internal injury and is often accompanied by attenuated motor reflexes and signs of autonomic dysfunction such as nausea, sweating and pallor. Visceral pain may be associated with secondary pain referred to the body surface with or without cutaneous or muscular hypo- or hyperalgesia. Acute visceral pain may result from various etiological factors including inflammation, infection, mechanical wall 
distress, ischemia and alterations in nerve function. While chronic visceral pain may derive from similar type stimuli, it may also occur in the absence of a well-defined stimulus. ${ }^{8}$

Chronic, recurrent abdominal pain is multi-dimensional and is strongly influenced by cognitive-emotional processes. ${ }^{9}$ Furthermore, a clinical characteristic of abdominal pain in IBS is that the symptom can be elicited and aggravated following certain stimuli, such as intake of (specific) nutrients or stress (see also Figure 8.1). ${ }^{10}$ Ideally, these characteristics should be taken into account when assessing abdominal pain in IBS patients.

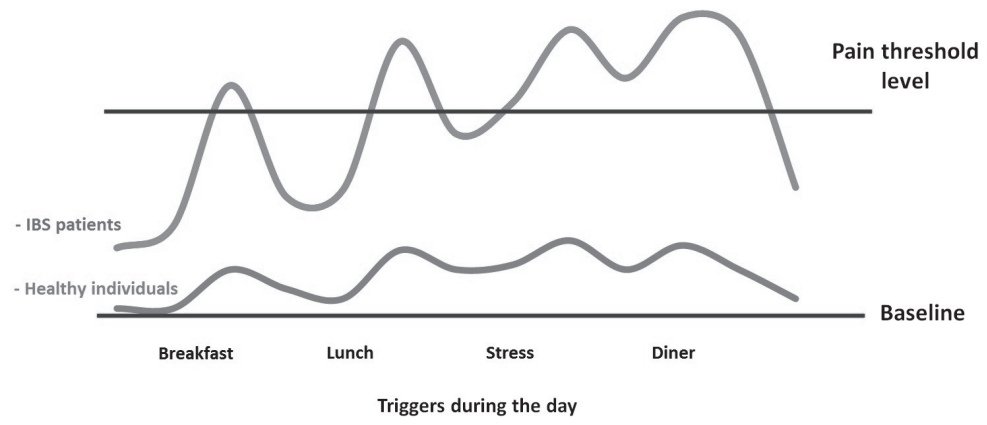

Figure 8.1 A theoretical representation of the fluctuation of abdominal pain during the day in IBS patients and healthy individuals, due to triggers such as food intake or daily stress.

The Initiative on Methods, Measurement, and Pain Assessment in Clinical Trials (IMMPACT) frequently publishes recommendations for design, provides considerations and outcome domains for clinical trials addressing chronic somatic pain. ${ }^{11}$ However, currently no consensus has been reached with regard to useful and relevant functional outcome measures for chronic abdominal pain to be used in clinical trials. Therefore, the aim of this study is to extensively review the published literature on methodology for measurement of recurrent chronic abdominal pain in IBS patients.

We sought to answer the following questions by systematically reviewing current literature:

1. Which tools for the assessment of abdominal pain in IBS patients are currently available?

2. Are these assessment tools of a context, content and construct validity and are they reproducible for the assessment of recurrent chronic abdominal pain and monitoring of efficacy of pain treatment in patients with IBS? 


\section{Methods}

\section{Search strategy}

A structured search in the PubMed / Medline databases was performed, using the following MeSH terms: (pain measurement OR pain assessment) AND (irritable bowel syndrome $O R$ abdominal pain $O R$ visceral pain). The following filters were used: Humans; English; Adult: 19+ years. Additional search terms, i.e. pain questionnaires, pain scales, pain trigger, pain aggravator, functional bowel disease, have been used as verification of the search results. Studies assessing non-Gl related pain and pain caused by malignancy or inflammatory disease, were excluded, due to neurobiological differences in pain processing and clinical presentation.

\section{Article selection}

Evaluation of articles and quality assessment was performed by two independent researchers (Z.M. and D.K.). All articles were screened based on title and abstract. Articles meeting the inclusion criteria were selected for full-text review. References of selected articles were scanned and if suitable included. All methods used to measure abdominal pain in patients with IBS were listed and if available, evidence of validity and reliability evaluated. Both reviewers agreed on inclusion of the specific articles. In case of disagreement a third reviewer (A.M.) decided regarding the inclusion.

The outcomes of interest with regard to this review were the following parameters: the method of pain measurement in IBS patients, the content and construct validity, internal consistency, test-retest reliability and responsiveness of quantification of chronic abdominal pain and monitoring of efficacy of abdominal pain treatment in IBS patients.

Content validity refers to the extent to which the instrument measures the symptoms that are indicative to its objective. ${ }^{12}$ Construct validity indicates to the degree of correlation between related instruments measuring the same symptoms (e.g. abdominal pain), expressed by the Pearson product-moment correlation coefficient and referred to as Pearson's $r^{13,14}$ Internal consistency, expressed by Cronbach $\alpha$ coefficient, indicates the correlation between different items of the same instrument, which are expected to measure a similar or the same general construct. ${ }^{15}$ Responsiveness is the ability to detect meaningful change in symptoms over time, while the test-retest reliability, also referred to as repeatability or retest reliability, often expressed by the intraclass correlation coefficient (ICC) or by the Cronbach $\alpha$, indicates the correlation between the same test items, scored under the same conditions, but at different time points. ${ }^{16}$ 


\section{Results}

An extensive search was performed to review the available literature on methods to measure recurrent chronic abdominal pain with focus on IBS. The number of hits was 533 (January 1987 - June 2015). After abstract and full text review 110 articles were included.

The reports included in this review can roughly be divided in four different categories of instruments to assess abdominal pain: (i) single-item (unidimensional) measures, (ii) multi-item measures, which can be uni- or multidimensional; i.e. general pain inventories, specific pain-dimension inventories, and general Gl-symptom severity questionnaires, (iii) 'real time' or 'momentary' assessment symptoms, and (iv) provocation tests. Several studies have combined more than one instrument.

\section{Single item measure of pain intensity (Table 8.1)}

Unidimensional, single-item measures of abdominal pain intensity are widely used and are considered accepted methods to measure acute pain in general. ${ }^{17}$ The list of the included manuscripts, using three different single-item instruments for the assessment of abdominal pain is shown in Table 8.1. All three methods have been used for the assessment of one dimension of pain in IBS patients, i.e. the intensity.

- Visual Analogue Scale (VAS) ${ }^{18-32}$ is a simple, fast and easy-to-use method to measure pain. A $100 \mathrm{~mm}$ horizontal line is anchored by two (verbal) descriptions: $0 \mathrm{~mm}$ indicating 'no pain' and $100 \mathrm{~mm}$ indicating 'worst pain bearable'. Three studies used a VAS with six severity descriptors under the VAS-line. The recall period varies across studies included in this review. The VAS is frequently used in studies to assess abdominal pain in IBS patients, but it is not psychometrically tested in the target population.

- Numeric Rating Scale (NRS) $)^{1,21,33-46}$ is a segmented numeric scale, using whole numbers as possible options, with various iterations, with the lowest value of 0 or 1 as 'no pain' to the maximum number as 'worst pain bearable'. The included studies in this review used a 5-point, 9-point, 10-point, 11-point and 21-point NRS for the assessment of abdominal pain. The NRS were used for repeated daily pain assessment (diaries) with a recall period of one day, or for a single pain assessment with longer recall periods. In particular the 10-point NRS has been well psychometrically tested in IBS patients, showed appropriate change in response to treatment and correlated well to the overall Gl symptom severity. The construct and discriminant validity, as well as the clinical responsiveness of the abdominal pain NRS was reported as 'excellent'. ${ }^{35}$

- Verbal rating Scale (VRS) ${ }^{47}$ is a verbal variant of the NRS or uses verbal ordinal descriptions of pain. It is favoured in situations when patients are not able to fill in a 
questionnaire, due to the medical condition or when using telecom interview methods.

Table 8.1 Unidimensional, single-item measure of pain intensity, for the assessment of abdominal pain in IBS.

\begin{tabular}{|c|c|c|c|c|}
\hline $\begin{array}{l}\text { Assessment } \\
\text { method }\end{array}$ & $\begin{array}{l}\text { Validity and } \\
\text { reliability }\end{array}$ & $\begin{array}{l}\text { Correlation to other } \\
\text { abdominal pain } \\
\text { measurement tools }\end{array}$ & Strengths & Limitations \\
\hline $\begin{array}{l}\text { Visual } \\
\text { Analogue } \\
\text { Scale } \\
\text { (VAS) }^{18-32}\end{array}$ & $\begin{array}{l}\text { No validity and } \\
\text { reliability evidence } \\
\text { available for chronic } \\
\text { abdominal pain. }\end{array}$ & $\begin{array}{l}\text { No correlations to other } \\
\text { measures of abdominal } \\
\text { pain reported. }\end{array}$ & $\begin{array}{l}\text { Simple, fast and } \\
\text { easy-to-use. } \\
\text { Appropriate for } \\
\text { illiterate subjects. }\end{array}$ & $\begin{array}{l}\text { Not psychometrically } \\
\text { tested for } \\
\text { measurement of } \\
\text { chronic abdominal pain } \\
\text { in IBS patients. } \\
\text { Measures only one } \\
\text { dimension of pain: } \\
\text { intensity. }\end{array}$ \\
\hline $\begin{array}{l}\text { Numeric } \\
\text { Rating Scale } \\
(\text { NRS) })^{1,21,33-46}\end{array}$ & $\begin{array}{l}\text { Construct and } \\
\text { discriminant validity } \\
\text { and clinical } \\
\text { responsiveness for } \\
\text { an end-of-day } \\
\text { abdominal pain } \\
\text { 10-point NRS was } \\
\text { reported as } \\
\text { 'excellent'. }\end{array}$ & $\begin{array}{l}\text { - 11-point NRS to SF- } \\
\text { MPQ sensory and } \\
\text { affective dimension of } \\
\text { pain: } r=0.63 \text { and } 0.48 \\
\text { - 11-point NRS to PDS: } \\
r=0.28(44) \\
\text { - 10-point NRS to IBS- } \\
\text { SSS: } r=0.60 \\
\text { - } 10 \text {-point NRS to FBDSI: } \\
r=0.49 \\
\text { - } 10 \text {-point NRS to VSI: } \\
r=0.28^{35}\end{array}$ & $\begin{array}{l}\text { Well } \\
\text { psychometrically } \\
\text { tested. Simple, fast } \\
\text { and easy to use. } \\
\text { Good correlation to } \\
\text { sensory dimension of } \\
\text { pain (SF-MPQ) and } \\
\text { GI-symptom severity } \\
\text { (IBS-SSS). }\end{array}$ & $\begin{array}{l}\text { Measures only one } \\
\text { dimension of pain: } \\
\text { intensity. }\end{array}$ \\
\hline $\begin{array}{l}\text { Verbal rating } \\
\text { Scale (VRS) }\end{array}$ & $\begin{array}{l}\text { No validity and } \\
\text { reliability evidence } \\
\text { available for chronic } \\
\text { abdominal pain. }\end{array}$ & $\begin{array}{l}\text { No correlations to other } \\
\text { measures of abdominal } \\
\text { pain reported. }\end{array}$ & $\begin{array}{l}\text { Simple, fast and easy } \\
\text { to use. } \\
\text { Subjects react } \\
\text { verbally. }\end{array}$ & $\begin{array}{l}\text { Not psychometrically } \\
\text { tested for } \\
\text { measurement of } \\
\text { chronic abdominal } \\
\text { pain. Measures only } \\
\text { one dimension of pain: } \\
\text { intensity. }\end{array}$ \\
\hline
\end{tabular}

\section{Multi-item questionnaires to assess pain}

More complex, multi-item questionnaires on pain and pain related factors are common in studies assessing GI symptoms in IBS patients. The list of the included articles, using 15 different multi-item questionnaires for the assessment of one or more dimensions of abdominal pain or Gl-symptom severity are shown in Table 8.2A and 8.2B.

\section{General pain questionnaires and assessment of specific domains of pain (Table 8.2A)}

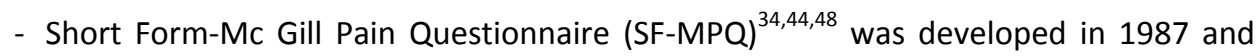
consists of 15 descriptors, i.e. 11 for sensory and 4 for affective dimension of pain, which are rated on an intensity scale as 0 to $3 .^{49}$ 
- Brief Pain Inventory (BPI) ${ }^{50}$ is developed in 1994 to measure cancer pain. Using a 10-point NRS the BPI assesses pain intensity at the time of responding and during the last week as well as the effect of pain on daily function of the subjects. ${ }^{51}$

- Pain Severity Scale of the West Haven Yale Multidimensional Pain Inventory (WHYMPI)(52) is developed in 1985 and consists of 52-items, using a 7-point NRS response scale. The recall period varies per question. Most items are based on a recall period of one week. The questionnaire is divided into 3 parts focussing on pain experience, including pain severity, the perceived response of others to patient's pain and the effect of pain on daily life. ${ }^{53}$

- Pain Discomfort Scale (PDS) $)^{34,44}$ is a 10-item instrument with a 5-point response scale. It has been developed in 1991 to briefly measure the chronic pain affect, i.e. painrelated suffering. The questions are not based on a specific recall period. ${ }^{54}$ The scale has not been tested psychometrically in IBS patients.

- Pain Vigilance and Awareness Questionnaire (PVAQ) ${ }^{38}$ is a 16-item measure assessing pain awareness on a 6-point scale, developed in 1997, focusing on awareness, vigilance, preoccupation and observation of pain in general, which may influence perceived pain severity and other pain responses. ${ }^{55}$ The scale is not psychometrically tested in IBS patients.

- Visceral Sensitivity Index (VSI) ${ }^{33,35,36,38,56}$ is a 15-item questionnaire scored on a 6point Likert scale, developed and validated in an IBS population to assess GIsymptom specific anxiety and hypervigilance that may accompany misappraisals of Gl-specific sensation and discomfort. ${ }^{33,56}$

Evidence for the use of the listed multi-item questionnaires for the assessment of pain (Table 8.2A):

Several general pain questionnaires assessing multiple dimensions of somatic chronic pain have been developed over the years. Some of these instruments have been used to assess abdominal pain in IBS patients (i.e. SF-MPQ, BPI, Pain Severity Scale of WHYMPI). However these questioners lack validation for this indication.

Chronic pain consists of a multitude of dimensions. A model of pain was described by Melzack et al. in 1968, stating that psychological properties of pain could be divided in sensory-discriminative, motivational-affective and cognitive-evaluative dimensions. ${ }^{9}$ Questionnaires focusing on the latter two dimensions of pain have been used in studies included in this review. Measuring a related construct, the PDS correlated significantly to sensory pain ( $r=0.28)$ and affective pain measurement by the SF-MPQ $(r=0.42)$, as well as to measurement of pain intensity by an 11-point NRS ( $r=0.28)$ in IBS patients. $^{34,44}$ This is also true for the VSI, which has been tested in IBS patient groups. The scale significantly correlated to abdominal pain intensity $(r=0.35)$ as assessed by a 10-point NRS in IBS patients. ${ }^{35}$ The concurrent, divergent and discriminant validity of the VSI was reported as 'good'. ${ }^{33,56}$

The PVAQ has not been psychometrically tested in an IBS patient population. 


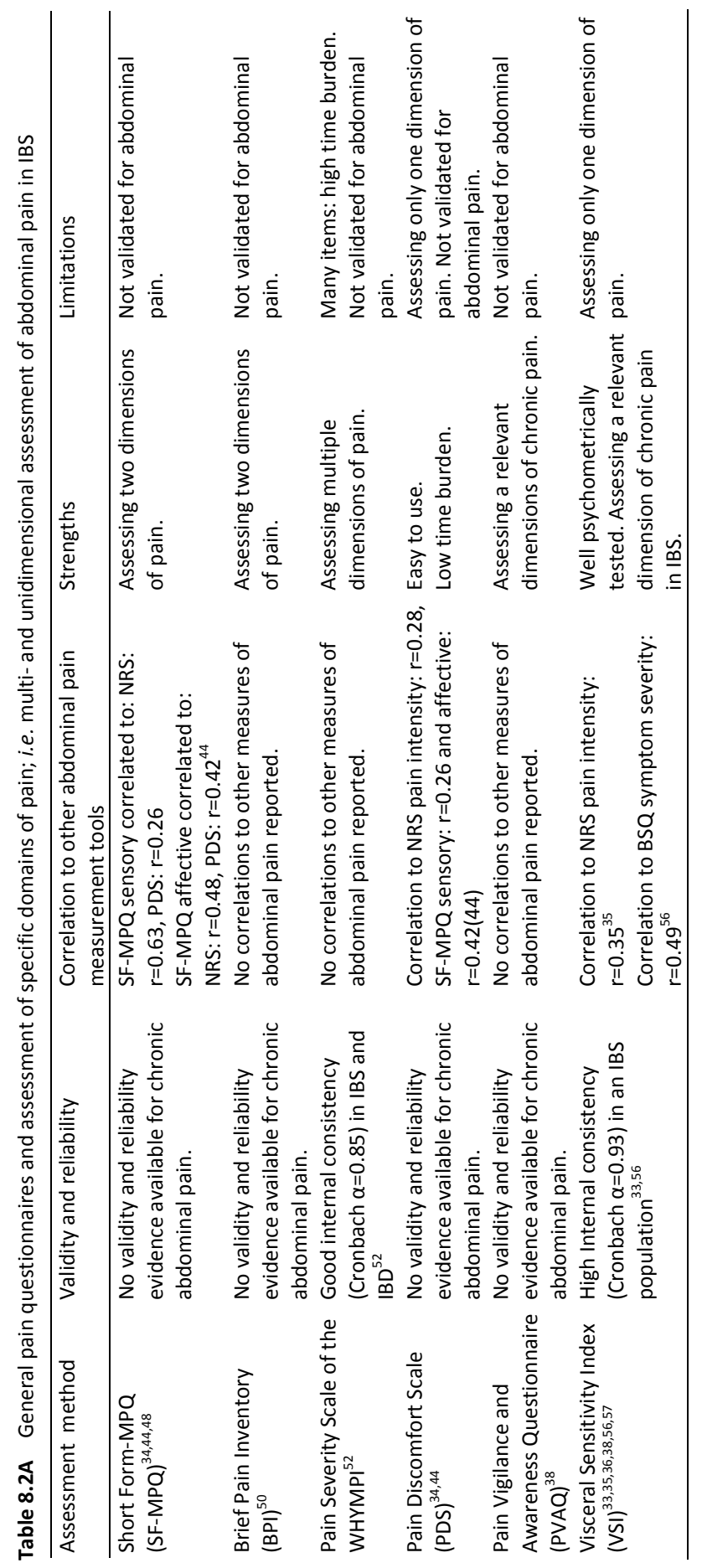




\section{Gl-symptom severity questionnaires (Table 8.2B):}

- Visual Analogue Scales for IBS (VAS-IBS) ${ }^{58,59}$ has been developed and psychometrically tested in 2007 as a short, patient reported questionnaire to be used in clinical practice to assess the status over time for patients with established IBS. It consists of 9 VAS scales, assessing bowel habits, bloating and flatulence, vomiting and nausea, psychological well-being, the influence on quality of life (QoL) and one VAS scale assessing the intensity of abdominal pain, with a one-week recall period. The VAS-IBS can be considered a questionnaire scoring general IBS symptoms, with a single question focusing on abdominal pain.

- Gastrointestinal Symptom Rating Scale (GSRS), ${ }^{25,58,60-66}$ the original version, is a 15-item rating scale with 4 possible answers per question with a recall period of one week. It has been developed in 1988 as an interview-based rating scale for the assessment of $\mathrm{GI}$ symptoms in patients with IBS and patients with peptic ulcer disease. ${ }^{60}$ It was later modified to become a self-administered questionnaire, with a 7-point scale used to assess GI symptoms. ${ }^{67}$ Only the first question focuses on abdominal pain, the other 14 items assess other GI symptoms.

- Gastrointestinal Symptom Rating Scale - IBS (GSRS-IBS), ${ }^{68,69}$ developed in 2003, is an IBS specific GSRS consisting of 13 items with a 7-point response scale. It assesses IBSrelated symptoms of which the first two questions focus on abdominal pain in the past week. $^{68}$

- IBS-Symptom Severity Scale (IBS-SSS), ${ }^{35,36,41,70-75}$ has been developed in 1997 and is a frequently used symptom assessment method in IBS trials and cohorts. It consists of VAS scales, open and binary questions and a figure of the abdomen. There are 9 items with several sub-items, of which 4 are related to abdominal pain, including intensity, frequency and location of pain, but also the relation of pain to stool pattern. $^{70}$

- Functional Bowel Disorder Severity Index (FBDSI) ${ }^{35,36,76}$ was developed in 1995 to be used for the selection of patients for research protocols (mild, moderate, severe FBD) but also for follow up to quantify clinical outcome over time. The score consists of three easy to obtain variables: current pain by VAS, diagnosis of chronic functional abdominal pain and the number of physician visits over the previous months. ${ }^{76}$

- Bowel Disease Questionnaire $(B D Q)^{77-80}$ was developed in 1989 to differentiate between non-ulcer dyspepsia, IBS and organic GI disease. It consists of $46 \mathrm{GI}$ symptom related items, with only 2 questions assessing the severity and frequency of abdominal pain in the previous two weeks, scored on a 4-point scale. ${ }^{77}$

- Bowel Symptom Questionnaire (BSQ) $)^{56,81-83}$ was developed in 1995. It is an extensive questionnaire of 95 items, with a broad focus, evaluating demographic characteristics, musculoskeletal symptoms, sleep pattern, appetite, sexual function and general Gl symptoms, including abdominal pain. The items are rated on a 20-point ordinal scale. ${ }^{81}$ 
- Symptom severity measures for IBS with constipation (IBS-C SSM) ${ }^{75}$ has been developed in 2014 for specific use in trials targeting IBS-C patients. It consists of five daily measures, i.e. abdominal pain, abdominal discomfort, bloating, abdominal fullness and abdominal cramping. The items are scored on an 11-point NRS. ${ }^{75}$

- Birmingham IBS symptom scale ${ }^{84,85}$ has been developed in 2008 as a Gl-symptom severity measure for IBS and was based on the on the Rome II criteria. It consists of 11 items, which are scored on a 6-point Likert scale, with a 4-week recall period. One of the 14 items measures the frequency of abdominal pain, while the other 13 assess the stool pattern and its relation to abdominal pain.

\section{Evidence for the use of the Gl-symptom severity questionnaires (Table 8.2B):}

A multitude of instruments are currently used in studies that include IBS patients to assess Gl-symptom severity. It has to be noted that most Gl-specific questionnaires are psychometrically tested in the target populations as measures of overall Gl-symptom severity, and not as measures of abdominal pain. Most Gl-symptom severity questionnaires contain only one or two items on abdominal pain, assessing intensity or frequency of pain, and may therefore be less suitable for studies focussing on abdominal pain as primary outcome measure. However, the IBS-SSS, a frequently used questionnaire, includes several items related to abdominal pain, i.e. intensity, frequency per 10 days, location and relation to stool pattern, and has been well psychometrically tested in IBS patients. ${ }^{70}$ The full list of Gl-symptom severity questionnaires and available data on validity, reliability and responsiveness for the assessment of abdominal pain is presented in Table 8.2B.

\section{"Real time" assessment of symptoms, including pain}

Ecological Momentary Assessment (EMA), also referred to as Experience Sampling Method (ESM), assesses symptoms via a digital device (comparable to a mobile phone), carried by the participants for several days during the time they are awake. The device emits an auditory signal randomly and repeatedly and participants are expected to fill in questions on the device at that particular moment. The questions, that need to be scored on a Likert scale, always relate to current symptoms (i.e. real time or momentary symptom assessment), thereby eliminating recall bias.

\section{Evidence for the use of 'real time' assessment of pain}

EMA / ESM was used only in two studies assessing overall GI symptoms in IBS patients. Apart from the EMA / ESM the subjects filled in a retrospective daily symptom diary. The ratings of abdominal pain in this diary overestimated levels reported by EMA. ${ }^{66,88}$ The EMA or ESM as a method as well as the selected questions to be used still have to be validated for the assessment of abdominal pain and other GI symptoms in IBS patients. 


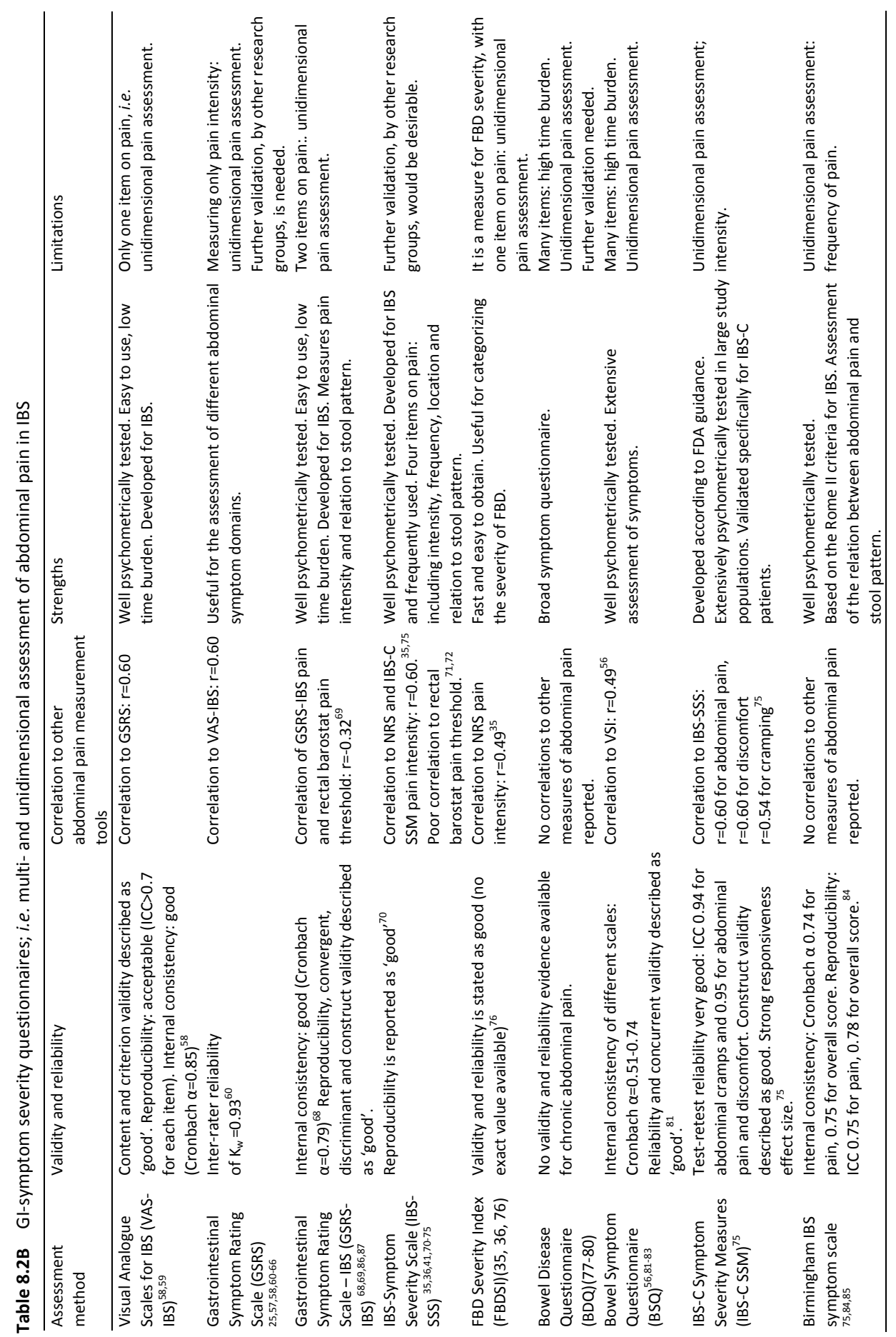




\section{Assessment of abdominal symptoms by provocation tests (Table 8.3):}

Provocation tests for the evaluation of visceral sensitivity in patient groups and healthy controls have become an accepted and frequently used method to discriminate hypersensitive from normosensitive individuals. It is important to note however, that given the acute nature of the intervention, these tests are generally not applicable for evaluation of chronic pain symptoms, but are rather indicative of alterations of perceptive responses to certain stimuli. Different methods have been developed and tested, i.e. mechanical intestinal stimulus (balloon distension, most frequently the rectum, using electronic barostat), nutrient or chemical provocation test (using a meal, acid or capsaicin), thermal or electric intestinal stimuli and thermal or electric stimuli on the skin combined with visceral stimuli. The intensity of provoked symptoms (pain, discomfort or urge) is assessed using a VAS or VRS.

The list of the different provocation tests, is shown in Table 8.3 and Supplementary information. Several studies have used more than one provocation test and have combined these with psychometric response scales.

\section{Evidence for the use of provocation tests for the assessment of abdominal pain (Table 8.3)}

It is under debate whether methods employing a provocation or trigger should be used to quantify chronic abdominal pain. Although several studies observed associations between increased symptom severity and the presence of visceral hypersensitivity in IBS patients, ${ }^{62,63,89}$ pain thresholds measured by rectal barostat only weakly correlate with spontaneous abdominal pain assessed by the GSRS ${ }^{69}$ or IBS-SSS. ${ }^{71,72}$ While changes in rectal perception post- versus pre-treatment have been observed in some studies, $^{90,91}$ others have not been able to observe any changes in rectal perception, ${ }^{61,65}$ questioning the role of provocation tests to assess abdominal pain and in particular response to treatment. 


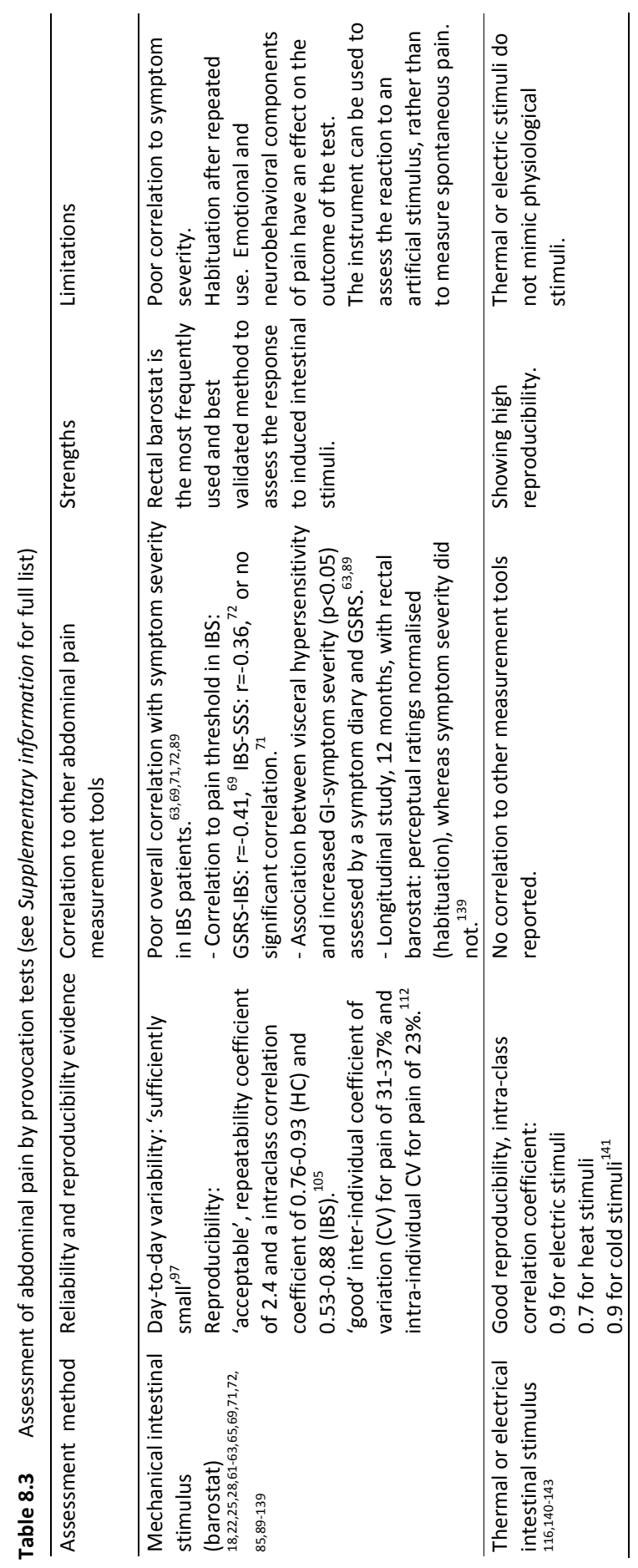




\section{Discussion}

In this review, the currently available instruments for assessment of chronic abdominal pain in IBS patients have been evaluated. A multitude of instruments is used in literature to assess chronic abdominal pain in this target population. This may in part be explained by the lack of generally accepted methods to assess chronic abdominal pain in IBS patientS. The large variety of assessment tools used in the reviewed studies is a limitation; the data from the different studies are difficult to compare and therefore cannot be pooled in a meta-analysis. Provocation tests, such as the rectal barostat, are often applied in studies that include IBS patients, and are useful tools to assess an acute response to artificial intestinal stimuli. However, the outcomes do not correlate well to chronic abdominal pain severity as assessed by retrospective questionnaires. Retrospective symptom assessment by paper questionnaires or diaries, has certain limitations, and in particular carries the risk of recall bias. Nevertheless, at this moment retrospective GI-symptom severity scales are frequently used, are easy to apply, and are the best validated methods for symptom assessment in IBS patients. However, these questionnaires in general include only the assessment of abdominal pain intensity. Momentary symptom assessment, may be an alternative that can eliminate recall bias when measuring abdominal pain, but it is a relatively new method in IBS studies, and validation of the construct of the questions used for momentary assessment is needed.

\section{Large variety of available questionnaires}

While unidimensional single-item measures, such as the VAS, NRS or VRS, are accepted methods to measure acute pain, to date, the evidence for their relevance in chronic abdominal pain assessment is only weak. However, the implementation of these methods, in particular the NRS, in a repeated measures approach (diaries) has proven to be useful. Therefore, the Food and Drugs Administration (FDA) has recommended the use of a 7-day mean of an end-of-day 11-point NRS as a primary endpoint for studies assessing abdominal pain in IBS patients. ${ }^{144}$ This method has been demonstrated to have the ability to identify responders to therapy in a number of large trials using linaclotide. ${ }^{40,46}$ However, post-hoc analysis of trial data have also shown that FDA Responder endpoints represent a rigorous and stringent standard, which is able to distinguish an efficacious therapeutic agent from a placebo - which is critically important in a field where the placebo-response rate has traditionally been quite high, but fails to provide adequate measures of overall drug efficacy. While responder endpoints may be useful for assessing if a treatment warrants FDA marketing approval, they provide limited information for the clinician to make treatment decisions specific to the individual patient's symptoms and may underestimate clinical response. ${ }^{145}$ Furthermore, there is no study published or available that has presented data on validity and reliability of the end-of-day 11-point NRS in IBS patients. However, an end- 
of-day 10-point NRS for abdominal pain has been psychometrically tested in a large IBS population with good results. ${ }^{35}$ Furthermore, using an end-of-day 11-point NRS, Lackner et al. have shown that questionnaires that take into account a longer recall period (i.e. a week) are more prone to recall bias compared to the end-of-day approach. ${ }^{45}$ This indicates that repeated measures over a certain period of time may provide a more representative impression of IBS symptom course, when compared to the end-of-week questionnaires. It needs to be acknowledged that measuring only one dimension of pain, i.e. intensity, as the VAS, VRS and NRS scales do, may be sufficient in specific medical conditions, such as acute cholecystitis. Chronic abdominal pain, however, is complex and includes more dimensions than only the intensity of pain. Therefore these instruments seem less useful for the measurement of chronic abdominal pain as they might not capture the complexity of pain perception IBS patients.

The multi-item questionnaires used in the reviewed articles focus on multiple pain dimensions (SF-MPQ, BDI, WHYMPI), on specific pain-related aspects (PDS, VSI, PVAQ), or on GI symptoms in general, including pain (VAS-IBS, GSRS, GSRS-IBS, IBS-SSS, FDBSI, BDQ, BSQ, IBS-C SSM, Birmingham IBS symptom scale). The general pain questionnaires have all been developed and psychometrically tested in patients with chronic somatic and/or neuropathic pain ${ }^{49,51,53,146,147}$ and have been implemented in studies assessing chronic abdominal pain in IBS without proper validation in this specific area. This also accounts for the questionnaires assessing pain discomfort (PDS) ${ }^{54}$ and pain vigilance and awareness (PVAQ). ${ }^{55}$ The VSI has been developed and validated for use in patients with $\mathrm{GI}$ disorders, and showed to be a valuable tool to measure anxiety related to $\mathrm{GI}$ symptoms, such as abdominal pain, in IBS patients. ${ }^{33,56}$ Endogenous stressors, such as Gl-symptom related anxiety, are part of the motivational-affective dimension of pain and play an important role in chronic abdominal pain. ${ }^{148}$

The Gl-symptom severity questionnaires have been developed for the assessment of GI symptoms in general and are psychometrically tested in IBS patients for this purpose. These questionnaires focus on a variety of symptoms, such as bowel habits, bloating, flatulence, vomiting, nausea, which are very relevant when monitoring effects of therapies in IBS patients. However, most GI-specific questionnaires do not focus enough on abdominal pain (i.e. measure only pain intensity) to be used as the only measurement in trials reporting pain as a primary or secondary outcome. Of these questionnaires (presented in Table 8.2B), the IBS-SSS includes the largest number of questions related to abdominal pain, i.e. intensity, frequency, location and relation to stool pattern. This questionnaire has been well psychometrically tested and is frequently used in IBS studies. Based on the current literature it appears to be the best retrospective instrument that can be used for the assessment of broader GI symptom severity in IBS patients, including abdominal pain. 
None of the available questionnaires include the assessment of possible triggers of abdominal pain when measuring symptoms. As therapies may focus on the abatement of a trigger, thereby preventing the occurrence of a pain episode instead of treating it, this may be a relevant topic in IBS trials. The difficulty however is that patients do not always consciously relate specific factors, such as diet or stress, to their symptoms, and thus cannot report this in a retrospective questionnaire.

\section{Limitations of retrospective questionnaires}

A major limitation of retrospective questionnaires, which are based on autobiographical memory, is recall bias, leading to abatement of accuracy of reports. Therefore, retrospective data, regardless of their content validity, may not be a reliable reflection of symptoms over a set period, but rather a reconstruction of a few specific moments. ${ }^{149}$ Studies have dealt with this issue by using end-of-day diaries or assessing shorter recall periods in questionnaires. ${ }^{35,45,64,89,150}$ Although the effect of recall bias can be decreased by these methods, ${ }^{45}$ the assessments are still based on the retrospective principle; asking responders to recall symptoms. In addition, assessment of chronic symptoms such as pain by retrospective questionnaires poses a second problem concerning ecological validity of the data. Symptoms and experiences may be affected and influenced by the circumstances in which they occur. Questionnaires are completed at a research facility or at home and these situations usually differ from moments when symptoms are at worst or from situations in which symptoms are triggered, therefore possible leading to bias by differences in environment. ${ }^{151}$ Furthermore, lack of patient compliance is an important disturbing factor in paper questionnaires. Studies that employed paper diaries in a population of patients with chronic somatic pain to measure symptoms have resulted in low compliance rates: $11 \%$ actual compliance and up to $80 \%$ fake compliance, presumably resulting from filling in diaries not during the indicated time window but afterwards. ${ }^{152}$ This limitation of fake compliance can be eliminated by the use of electronic patient reported outcomes instead of paper versions or by calling call into an interactive voice response system. ${ }^{145}$

\section{'Real time' assessment of pain; a better alternative?}

EMA or ESM, based on digital momentary or real life assessment of symptoms, is a relatively new method in this research field. ${ }^{66,88}$ The method may help to overcome the above described limitations of retrospective questionnaires related to recall bias. It has been validated and is frequently used in psychiatric and psychological studies. ${ }^{153}$ Variability of symptoms during the day (see Figure 8.1) can be assessed, since repeated measures are used. We have recently demonstrated that IBS patients report higher scores for abdominal pain in retrospective questionnaires compared to ESM, with a tendency to report peak rather than average pain scores, while this was not found for other GI-symptoms such as bloating, flatulence or belching. ${ }^{66}$ Furthermore, EMA / ESM 
may allow triggers of pain to be examined in more detail, by taking into account situations or symptoms, scored at the previous assessment moment, that have occurred prior to a pain episode. Furthermore, ecological bias may be reduced by adding questions that relate to the daily life situations or conditions at moments of increased symptom severity. However, a relevant limitation of this method is the higher patient burden, since subjects are expected to respond at multiple random moments during daily life. ${ }^{154}$ Therefore the number of questions should be restricted.

Although it is a promising tool, more research is needed to validate this method in the target population. A crucial step in development of this tool is the selection and validation of questions that are programmed into the EMA / ESM, since content, construct and criterion validity are dependent of the selected questions.

\section{Provocation tests; useful for the assessment of chronic abdominal pain?}

Provocation tests have been used to evaluate efficacy of therapies and for mechanistic studies, focussing on the pathophysiology of visceral pain and for the interaction with somatic pain in IBS patients. Provocation tests are currently the best available option to measure the response to visceral stimuli in human subjects and are therefore important for mechanistic research of visceral sensitivity. Rectal barostat using balloon distention is the most frequently used and best validated method in this field. It may be used in combination with methods to assess peripheral and central nervous system signal processing, e.g. $\mathrm{fMRI}$, or in combination with somatic pain stimuli (e.g. on the skin), to improve our understanding of visceral pain processing. Based on these studies, visceral hypersensitivity is considered by many as a biological marker or a 'hallmark' for IBS. $^{131}$

However, the exact role and value of provocation tests in the quantification of visceral pain intensity is not clear. These methods do not assess spontaneous pain, neither do they correlate well to symptom severity or to 'normal evoked pain' in the every-day life of IBS patients. ${ }^{69,89}$

In summary, the current level of evidence for the use of provocation tests in the assessment and follow-up of chronic abdominal pain in IBS patients is rather low. More insight into the pathophysiology of visceral sensations and development of a visceral pain model is required in order to be able to develop more adequate tools for objective assessment of chronic abdominal pain.

\section{Future steps}

An uniformly accepted model, combining and explaining the different dimensions of chronic abdominal pain in IBS is currently not available. For reasons of tool development for measuring responses to drug therapies and in order to be able to measure the full spectrum of this symptom in IBS patients (see also Table 8.4), a validated combination of instruments is needed that takes into account not only pain 
intensity but also other dimensions of chronic abdominal pain, as well as psychological symptoms, since these factors may fluctuate as well and may influence the pain sensation. $^{150,155}$ Furthermore, measures of Gl-symptom severity, assessment of aggravating and alleviating factors of pain, the impact of abdominal pain on daily life and the effect of extra-intestinal symptoms such as somatic pain, should be taken into account. It is important to recognise that all separate dimensions that have to be assessed by one or more tools, have to be validated separately. It should be noted that the concepts and items measured depend on the context of use. Depending on the main objective of a study, specific factors listed in Table 8.4 should be prioritised. Furthermore, while some domains of pain, as well as GI or psychological symptoms may fluctuate over time and therefore should be measured repeatedly, rather than once, other aspects as triggers of pain or the impact on daily life may be constant and could be measured only at baseline. For all factors listed in Table 8.4 questionnaires are currently available, but in some cases need to be adapted and validated for use in the target population.

Table 8.4 Measuring chronic abdominal pain in IBS patients: factors to be taken into account

\begin{tabular}{|c|c|}
\hline Multiple pain dimensions & Sensory, affective and evaluative aspects of chronic abdominal pain \\
\hline Associated symptoms & $\begin{array}{l}\text { Gl-related symptoms, e.g. bowel habits, bloating, flatulence, nausea, reflux, } \\
\text { abdominal cramps }\end{array}$ \\
\hline Possible triggers & Identifiable aggravating factors for abdominal pain, e.g. food intake, stress \\
\hline Possible alleviating factors & Factors causing relive of abdominal pain, e.g. defecating \\
\hline Psychological factors & $\begin{array}{l}\text { Anxiety and depression scores, as well as GI-related anxiety, neuroticism, } \\
\text { somatisation, vigilance, pain coping and cognitions }\end{array}$ \\
\hline Somatic pain & $\begin{array}{l}\text { Taking into account the evidence for general pain syndromes, assessment of } \\
\text { somatic pain should be added to measurement of chronic abdominal pain }\end{array}$ \\
\hline Impact of abdominal pain & Impact on daily life, quality of life in different domains \\
\hline \multicolumn{2}{|c|}{ Overall: recall bias should be eliminated and ecological validity should be ensured } \\
\hline
\end{tabular}

\section{Conclusion}

For the assessment of unidimensional abdominal pain intensity, the end-of-day 10-point NRS has been validated in IBS patients and proved to be a reliable tool to measure response to therapies. The best-validated instruments included in this review have been developed to assess general GI-symptom severity in IBS patients, of which the IBS-Symptom Severity Scale is best suited to assess abdominal pain. Furthermore, the Visceral Sensitivity Index is validated and useful to assess the psycho-emotional dimension related to among others abdominal pain in IBS patients.

Taken together, a multitude of different instruments is currently used to assess chronic abdominal pain in IBS patients, making the endpoints of published studies difficult to compare. There is need for a validated (existing or newly developed) instrument for the assessment of chronic abdominal pain, which takes into account multiple dimensions of pain in IBS patients, and reduces relevant limitations of currently available retrospective methods, such as recall bias. 


\section{References}

1. Spiegel B, Strickland A, Naliboff BD, Mayer EA, Chang L. Predictors of patient-assessed illness severity in irritable bowel syndrome. Am J Gastroenterol. 2008;103:2536-43.

2. IASP. International Association for the Study of Pain: Pain Terminology 2011. http://www.iasppain.org/AM/Template.cfm?Section=Pain_Defi...isplay.cfm\&ContentID=1728.

3. Treede RD, Rief W, Barke A, Aziz Q, Bennett MI, Benoliel R, et al. A classification of chronic pain for ICD11. Pain. 2015;156:1003-7.

4. Longstreth GF, Thompson WG, Chey WD, Houghton LA, Mearin F, Spiller RC. Functional bowel disorders. Gastroenterology. 2006;130:1480-91.

5. Fornasari D. Pain mechanisms in patients with chronic pain. Clin Drug Investig. 2012;32 Suppl 1:45-52.

6. Cervero F. Visceral versus somatic pain: similarities and differences. Dig Dis. 2009;27 Suppl 1:3-10.

7. Loeser JD, Treede RD. The Kyoto protocol of IASP Basic Pain Terminology. Pain. 2008;137:473-7.

8. Keszthelyi D, Troost FJ, Simren M, Ludidi S, Kruimel JW, Conchillo JM, et al. Revisiting concepts of visceral nociception in irritable bowel syndrome. Eur J Pain. 2012;16:1444-54.

9. Melzack R. Sensory, motivational and central control determinants of pain: A new conceptual model. The Skin Senses Springfield, IL, Charles C Thomas1968. p. 423-43.

10. Simren M, Mansson A, Langkilde AM, Svedlund J, Abrahamsson H, Bengtsson U, et al. Food-related gastrointestinal symptoms in the irritable bowel syndrome. Digestion. 2001;63:108-15.

11. Dworkin RH, Turk DC, Peirce-Sandner S, Burke LB, Farrar JT, Gilron I, et al. Considerations for improving assay sensitivity in chronic pain clinical trials: IMMPACT recommendations. Pain. 2012;153:1148-58.

12. Lawshe $\mathrm{CH}$. A quantitative approach to content validity. Personnel Psychology. 1975;28:563-75.

13. Campell DTaF, D. W. Convergent and discriminant validation by the multitrait-multimethod matrix. Psychological Bulletin. 1959;56:81-105.

14. Pearson K. On further methods of determining correlation. London: Dulau and Co Cambridge: Draper's Company Research Memoirs (Biometric Series IV). 1907;39.

15. Cronbach $\amalg$, Warrington WG. Time-limit tests: estimating their reliability and degree of speeding. Psychometrika. 1951;16:167-88.

16. Atkinson G, Nevill AM. Statistical methods for assessing measurement error (reliability) in variables relevant to sports medicine. Sports Med. 1998;26:217-38.

17. Hjermstad MJ, Fayers PM, Haugen DF, Caraceni A, Hanks GW, Loge JH, et al. Studies comparing Numerical Rating Scales, Verbal Rating Scales, and Visual Analogue Scales for assessment of pain intensity in adults: a systematic literature review. J Pain Symptom Manage. 2011;41:1073-93.

18. Guthrie E, Barlow J, Fernandes L, Ratcliffe J, Read N, Thompson DG, et al. Changes in tolerance to rectal distension correlate with changes in psychological state in patients with severe irritable bowel syndrome. Psychosom Med. 2004;66:578-82.

19. Tanum L, Malt UF. Personality and physical symptoms in nonpsychiatric patients with functional gastrointestinal disorder. J Psychosom Res. 2001;50:139-46.

20. Saggioro A. Probiotics in the treatment of irritable bowel syndrome. J Clin Gastroenterol.2004;38(6 Suppl):S104-6.

21. Chassany O, Bonaz B, Bruley DESVS, Bueno L, Cargill G, Coffin B, et al. Acute exacerbation of pain in irritable bowel syndrome: efficacy of phloroglucinol/trimethylphloroglucinol. A randomized, doubleblind, placebo-controlled study. Aliment Pharmacol Ther. 2007;25:1115-23.

22. Simren M, Agerforz $\mathrm{P}$, Bjornsson ES, Abrahamsson H. Nutrient-dependent enhancement of rectal sensitivity in irritable bowel syndrome (IBS). Neurogastroenterol Motil.2007;19:20-9.

23. Muller-Lissner SA, Fumagalli I, Bardhan KD, Pace F, Pecher E, Nault B, et al. Tegaserod, a 5-HT(4) receptor partial agonist, relieves symptoms in irritable bowel syndrome patients with abdominal pain, bloating and constipation. Aliment Pharmacol Ther. 2001;15:1655-66.

24. Muller-Lissner S, Koch G, Talley NJ, Drossman D, Rueegg P, Dunger-Baldauf C, et al. Subject's Global Assessment of Relief: an appropriate method to assess the impact of treatment on irritable bowel syndrome-related symptoms in clinical trials. J Clin Epidemiol. 2003;56:310-6. 
25. Klooker TK, Kuiken SD, Lei A, Boeckxstaens GE. Effect of long-term treatment with octreotide on rectal sensitivity in patients with non-constipated irritable bowel syndrome. Aliment Pharmacol Ther. 2007;26:605-15.

26. Hellstrom PM, Hein J, Bytzer P, Bjornsson E, Kristensen J, Schambye H. Clinical trial: the glucagon-like peptide-1 analogue ROSE-010 for management of acute pain in patients with irritable bowel syndrome: a randomized, placebo-controlled, double-blind study. Aliment Pharmacol Ther. 2009;29:198-206.

27. Gonlachanvit S, Mahayosnond A, Kullavanijaya P. Effects of chili on postprandial gastrointestinal symptoms in diarrhoea predominant irritable bowel syndrome: evidence for capsaicin-sensitive visceral nociception hypersensitivity. Neurogastroenterol Motil.2009;21:23-32.

28. Khalif IL, Guigley EMM, Makarchuk PA, Golovenko OV, Podmarenkova LF, Dzhanayev YA. Interactions between Symptoms and Motor and Visceral Sensory Responses of Irritable Bowel Syndrome Patients to Spasmolytics (Antispasmodics). J Gastrointestin Liver Dis. 2009;18:17-22.

29. Kim ES, Cheon JH, Park JJ, Moon CM, Hong SP, Kim TI, et al. Colonoscopy as an adjunctive method for the diagnosis of irritable bowel syndrome: focus on pain perception. J Gastroenterol Hepatol. 2010;25:1232-8.

30. Imai A, Kato M, Ono S, Shimizu Y, Takeda H, Asaka M. Efficacy of carbon dioxide-insufflating colonoscopy in patients with irritable bowel syndrome: A randomized double-blind study. J Gastroenterol Hepatol. 2012;27:1623-8.

31. Farnam A, Somi MH, Farhang S, Mahdavi N, Ali Besharat M. The therapeutic effect of adding emotional awareness training to standard medical treatment for irritable bowel syndrome: a randomized clinical trial. J Psychiatr Pract. 2014;20:3-11.

32. Halmos EP, Power VA, Shepherd SJ, Gibson PR, Muir JG. A diet low in FODMAPs reduces symptoms of irritable bowel syndrome. Gastroenterology. 2014;146:67-75 e5.

33. Labus JS, Mayer EA, Chang L, Bolus R, Naliboff BD. The central role of gastrointestinal-specific anxiety in irritable bowel syndrome: further validation of the visceral sensitivity index. Psychosom Med. 2007;69:89-98.

34. Lackner JM, Jaccard J, Blanchard EB. Testing the sequential model of pain processing in irritable bowel syndrome: a structural equation modeling analysis. Eur J Pain. 2005;9:207-18.

35. Spiegel B, Bolus R, Harris LA, Lucak S, Naliboff B, Esrailian E, et al. Measuring irritable bowel syndrome patient-reported outcomes with an abdominal pain numeric rating scale. Aliment Pharmacol Ther. 2009;30:1159-70.

36. Spiegel BMR, Bolus R, Harris LA, Lucak S, Chey WD, Sayuk G, et al. Characterizing abdominal pain in IBS: guidance for study inclusion criteria, outcome measurement and clinical practice. Aliment Pharmacol Ther. 2010;32:1192-202.

37. Masand PS, Pae CU, Krulewicz S, Peindl K, Mannelli P, Varia IM, et al. A double-blind, randomized, placebo-controlled trial of paroxetine controlled-release in irritable bowel syndrome. Psychosomatics. 2009;50:78-86.

38. Craske MG, Wolitzky-Taylor KB, Labus J, Wu S, Frese M, Mayer EA, et al. A cognitive-behavioral treatment for irritable bowel syndrome using interoceptive exposure to visceral sensations. Behav Res Ther. 2011;49:413-21.

39. Hellstrom PM, Saito YA, Bytzer P, Tack J, Mueller-Lissner S, Chang L. Characteristics of acute pain attacks in patients with irritable bowel syndrome meeting Rome III criteria. Am J Gastroenterol. 2011;106:1299-307.

40. Rao S, Lembo AJ, Shiff SJ, Lavins BJ, Currie MG, Jia XD, et al. A 12-week, randomized, controlled trial with a 4-week randomized withdrawal period to evaluate the efficacy and safety of linaclotide in irritable bowel syndrome with constipation. Am J Gastroenterol. 2012;107:1714-24; quiz p 25.

41. Tuteja AK, Fang JC, Al-Suqi M, Stoddard GJ, Hale DC. Double-blind placebo-controlled study of mesalamine in post-infective irritable bowel syndrome - a pilot study. Scand J Gastroenterol. 2012;47:1159-64.

42. Bouchoucha M, Fysekidis M, Devroede G, Raynaud JJ, Bejou B, Benamouzig R. Abdominal pain localization is associated with non-diarrheic Rome III functional gastrointestinal disorders. Neurogastroenterol Motil. 2013;25:686-93.

43. Lacy BE, Wang F, Bhowal S, Schaefer E, study g. On-demand hyoscine butylbromide for the treatment of self-reported functional cramping abdominal pain. Scand J Gastroenterol. 2013;48:926-35. 
44. Lackner JM, Quigley BM. Pain catastrophizing mediates the relationship between worry and pain suffering in patients with irritable bowel syndrome. Behaviour research and therapy. 2005;43:943-57.

45. Lackner JM, Jaccard J, Keefer L, Firth R, Carosella AM, Sitrin M, et al. The accuracy of patient-reported measures for $\mathrm{Gl}$ symptoms: a comparison of real time and retrospective reports. Neurogastroenterol Motil.2014;26:1802-11.

46. Chey WD, Lembo AJ, Lavins BJ, Shiff SJ, Kurtz CB, Currie MG, et al. Linaclotide for irritable bowel syndrome with constipation: a 26-week, randomized, double-blind, placebo-controlled trial to evaluate efficacy and safety. Am J Gastroenterol. 2012;107:1702-12.

47. Brown PM, Drossman DA, Wood AJ, Cline GA, Frazier KS, Jackson JI, et al. The tryptophan hydroxylase inhibitor LX1031 shows clinical benefit in patients with nonconstipating irritable bowel syndrome. Gastroenterology. 2011;141:507-16.

48. Akbar A, Yiangou Y, Facer P, Brydon WG, Walters JR, Anand P, et al. Expression of the TRPV1 receptor differs in quiescent inflammatory bowel disease with or without abdominal pain. Gut. 2010;59:767-74.

49. Melzack R. The short-form McGill Pain Questionnaire. Pain. 1987;30:191-7.

50. Brennan BP, Fogarty KV, Roberts JL, Reynolds KA, Pope HG, Jr., Hudson JI. Duloxetine in the treatment of irritable bowel syndrome: an open-label pilot study. Hum Psychopharmacol. 2009;24:423-8.

51. Cleeland CS, Ryan KM. Pain assessment: global use of the Brief Pain Inventory. Ann Acad Med Singapore. 1994;23:129-38.

52. Seres G, Kovacs Z, Kovacs A, Kerekgyarto O, Sardi K, Demeter P, et al. Different associations of health related quality of life with pain, psychological distress and coping strategies in patients with irritable bowel syndrome and inflammatory bowel disorder. J Clin Psychol Med Settings. 2008;15:287-95.

53. Kerns RD, Turk DC, Rudy TE. The West Haven-Yale Multidimensional Pain Inventory (WHYMPI). Pain. 1985;23:345-56.

54. Jensen MP, Karoly $\mathrm{P}$, Harris P. Assessing the affective component of chronic pain: development of the Pain Discomfort Scale. J Psychosom Res. 1991;35:149-54.

55. McCracken LM. 'Attention' to pain in persons with chronic pain: a behavioural approach. Behav Ther. 1997;28:271-84.

56. Labus JS, Bolus R, Chang L, Wiklund I, Naesdal J, Mayer EA, et al. The Visceral Sensitivity Index: development and validation of a gastrointestinal symptom-specific anxiety scale. Aliment Pharmacol Ther. 2004;20:89-97.

57. Jerndal P, Ringstrom G, Agerforz P, Karpefors M, Akkermans LM, Bayati A, et al. Gastrointestinalspecific anxiety: an important factor for severity of GI symptoms and quality of life in IBS. Neurogastroenterol Motil.2010;22:646-e179.

58. Bengtsson M, Ohlsson B, Ulander K. Development and psychometric testing of the Visual Analogue Scale for Irritable Bowel Syndrome (VAS-IBS). BMC gastroenterology. 2007;7:16

59. Bengtsson M, Hammar O, Mandl T, Ohlsson B. Evaluation of gastrointestinal symptoms in different patient groups using the visual analogue scale for irritable bowel syndrome (VAS-IBS). BMC Gastroenterology. 2011;11:122.

60. Svedlund J, Sjodin I, Dotevall G. GSRS--a clinical rating scale for gastrointestinal symptoms in patients with irritable bowel syndrome and peptic ulcer disease. Dig Dis Sci. 1988;33:129-34.

61. Kuiken SD, Tytgat GN, Boeckxstaens GE. The selective serotonin reuptake inhibitor fluoxetine does not change rectal sensitivity and symptoms in patients with irritable bowel syndrome: a double blind, randomized, placebo-controlled study. Clin Gastroenterol Hepatol. 2003;1:219-28.

62. Kuiken SD, Lindeboom R, Tytgat GN, Boeckxstaens GE. Relationship between symptoms and hypersensitivity to rectal distension in patients with irritable bowel syndrome. Aliment Pharmacol Ther. 2005;22:157-64.

63. Ludidi S, Mujagic Z, Jonkers D, Keszthelyi D, Hesselink M, Kruimel J, et al. Markers for visceral hypersensitivity in patients with irritable bowel syndrome. Neurogastroenterol Motil.2014;26:1104-11.

64. Mujagic Z, Ludidi S, Keszthelyi D, Hesselink MA, Kruimel JW, Lenaerts K, et al. Small intestinal permeability is increased in diarrhoea predominant IBS, while alterations in gastroduodenal permeability in all IBS subtypes are largely attributable to confounders. Aliment Pharmacol Ther. 2014;40:288-97. 
65. Ludidi S, Jonkers DM, Koning CJ, Kruimel JW, Mulder L, van der Vaart IB, et al. Randomized clinical trial on the effect of a multispecies probiotic on visceroperception in hypersensitive IBS patients. Neurogastroenterol Motil.2014;26:705-14.

66. Mujagic Z, Leue C, Vork L, Lousberg R, Jonkers DM, Keszthelyi D, et al. The Experience Sampling Method - a new digital tool for momentary symptom assessment in IBS: an exploratory study. Neurogastroenterol Motil.2015.

67. Revicki DA, Wood M, Wiklund I, Crawley J. Reliability and validity of the Gastrointestinal Symptom Rating Scale in patients with gastroesophageal reflux disease. Qual Life Res. 1998;7:75-83.

68. Wiklund IK, Fullerton S, Hawkey CJ, Jones RH, Longstreth GF, Mayer EA, et al. An irritable bowel syndrome-specific symptom questionnaire: development and validation. Scand J Gastroenterol. 2003;38:947-54.

69. Posserud I, Syrous A, Lindstrom L, Tack J, Abrahamsson H, Simren M. Altered rectal perception in irritable bowel syndrome is associated with symptom severity. Gastroenterology. 2007;133:1113-23.

70. Francis CY, Morris J, Whorwell PJ. The irritable bowel severity scoring system: a simple method of monitoring irritable bowel syndrome and its progress. Aliment Pharmacol Ther. 1997;11:395-402.

71. Sabate JM, Veyrac M, Mion F, Siproudhis L, Ducrotte P, Zerbib F, et al. Relationship between rectal sensitivity, symptoms intensity and quality of life in patients with irritable bowel syndrome. Aliment Pharmacol Ther. 2008;28:484-90.

72. Kanazawa M, Palsson OS, Thiwan SI, Turner MJ, van Tilburg MA, Gangarosa LM, et al. Contributions of pain sensitivity and colonic motility to IBS symptom severity and predominant bowel habits. Am J Gastroenterol. 2008;103:2550-61.

73. Sondergaard B, Olsson J, Ohlson K, Svensson U, Bytzer P, Ekesbo R. Effects of probiotic fermented milk on symptoms and intestinal flora in patients with irritable bowel syndrome: a randomized, placebocontrolled trial. Scand J Gastroenterol. 2011;46:663-72.

74. Annahazi A, Ferrier L, Bezirard V, Leveque M, Eutamene H, Ait-Belgnaoui A, et al. Luminal cysteineproteases degrade colonic tight junction structure and are responsible for abdominal pain in constipation-predominant IBS. Am J Gastroenterol. 2013;108:1322-31.

75. Williams VS, Nelson LM, Fehnel SE, MacDougall J, Carson RT, Tourkodimitris S, et al. Psychometric validation of symptom severity measures in irritable bowel syndrome with constipation. Aliment Pharmacol Ther. 2014;40:298-308.

76. Drossman DA, Li ZM, Toner BB, Diamant NE, Creed FH, Thompson D, et al. Functional Bowel Disorders a Multicenter Comparison of Health-Status and Development of Illness Severity Index. Dig Dis Sci. 1995;40:986-95.

77. Talley NJ, Phillips SF, Melton L, Wiltgen C, Zinsmeister AR. A Patient Questionnaire to Identify BowelDisease. Ann Intern Med. 1989;111:671-4.

78. Neri M, Laterza F, Howell S, Di Gioacchino M, Festi D, Ballone E, et al. Symptoms discriminate irritable bowel syndrome from organic gastrointestinal diseases and food allergy. Eur J Gastroenterol Hepatol. 2000;12:981-8.

79. Barbara G, Stanghellini V, De Giorgio R, Cremon C, Cottrell GS, Santini D, et al. Activated mast cells in proximity to colonic nerves correlate with abdominal pain in irritable bowel syndrome. Gastroenterology. 2004;126:693-702.

80. Talley NJ, Phillips SF, Bruce B, Twomey CK, Zinsmeister AR, Melton LJ, 3rd. Relation among personality and symptoms in nonulcer dyspepsia and the irritable bowel syndrome. Gastroenterology. 1990;99:327-33.

81. Talley NJ, Boyce PM, Owen BK, Newman P, Paterson KJ. Initial validation of a bowel symptom questionnaire and measurement of chronic gastrointestinal symptoms in Australians. Aus N Z J Med. 1995;25:302-8.

82. Schmulson M, Lee OY, Chang L, Naliboff B, Mayer EA. Symptom differences in moderate to severe IBS patients based on predominant bowel habit. Am J Gastroenterol. 1999;94:2929-35.

83. Tan S, Tillisch K, Bolus SR, Olivas TI, Spiegel BM, Naliboff B, et al. Traditional Chinese medicine based subgrouping of irritable bowel syndrome patients. Am J Chin Med. 2005;33:365-79.

84. Roalfe AK, Roberts LM, Wilson S. Evaluation of the Birmingham IBS symptom questionnaire. BMC Gastroenterology. 2008;8:30. 
85. Zhu Y, Wu Z, Ma X, Liu H, Bao C, Yang L, et al. Brain regions involved in moxibustion-induced analgesia in irritable bowel syndrome with diarrhea: a functional magnetic resonance imaging study. BMC Complement Altern Med. 2014;14:500.

86. Ljotsson B, Hesser H, Andersson E, Lackner JM, El Alaoui S, Falk L, et al. Provoking symptoms to relieve symptoms: a randomized controlled dismantling study of exposure therapy in irritable bowel syndrome. Behav Res Ther. 2014;55:27-39.

87. Fassov J, Lundby L, Worsoe J, Buntzen S, Laurberg S, Krogh K. A randomised, controlled study of small intestinal motility in patients treated with sacral nerve stimulation for irritable bowel syndrome. BMC Gastroenterology. 2014;14:111.

88. Weinland SR, Morris CB, Hu Y, Leserman J, Bangdiwala SI, Drossman DA. Characterization of episodes of irritable bowel syndrome using ecological momentary assessment. Am J Gastroenterol. 2011;106: 1813-20.

89. van der Veek PP, Van Rood YR, Masclee AA. Symptom severity but not psychopathology predicts visceral hypersensitivity in irritable bowel syndrome. Clin Gastroenterol Hepatol. 2008;6:321-8.

90. Delvaux M, Louvel D, Lagier E, Scherrer B, Abitbol JL, Frexinos J. The kappa agonist fedotozine relieves hypersensitivity to colonic distention in patients with IBS. Gastroenterology. 1999;116:38-45.

91. Lembo T, Naliboff BD, Matin K, Munakata J, Parker RA, Gracely RH, et al. Irritable bowel syndrome patients show altered sensitivity to exogenous opioids. Pain. 2000;87:137-47.

92. Ritchie J. Pain from distension of the pelvic colon by inflating a balloon in the irritable colon syndrome. Gut. 1973;14:125-32.

93. Whitehead WE, Holtkotter B, Enck P, Hoelzl R, Holmes KD, Anthony J, et al. Tolerance for rectosigmoid distention in irritable bowel syndrome. Gastroenterology. 1990;98:1187-92.

94. Mertz H, Naliboff B, Munakata J, Niazi N, Mayer EA. Altered rectal perception is a biological marker of patients with irritable bowel syndrome. Gastroenterology. 1995;109:40-52.

95. Munakata J, Naliboff B, Harraf F, Kodner A, Lembo T, Chang L, et al. Repetitive sigmoid stimulation induces rectal hyperalgesia in patients with irritable bowel syndrome. Gastroenterology. 1997;112: 55-63.

96. Naliboff BD, Munakata J, Fullerton S, Gracely RH, Kodner A, Harraf F, et al. Evidence for two distinct perceptual alterations in irritable bowel syndrome. Gut. 1997;41:505-12.

97. Hammer HF, Phillips SF, Camilleri M, Hanson RB. Rectal tone, distensibility, and perception: reproducibility and response to different distensions. Am J Physiol. 1998;274:G584-90.

98. Chang L, Munakata J, Mayer EA, Schmulson MJ, Johnson TD, Bernstein CN, et al. Perceptual responses in patients with inflammatory and functional bowel disease. Gut. 2000;47:497-505.

99. Chang FY, Lu CL, Chen CY, Luo JC. Efficacy of dioctahedral smectite in treating patients of diarrheapredominant irritable bowel syndrome. J Gastroenterol Hepatol. 2007;22:2266-72.

100. Simren M, Abrahamsson H, Bjornsson ES. An exaggerated sensory component of the gastrocolonic response in patients with irritable bowel syndrome. Gut. 2001;48:20-7.

101. Verne GN, Robinson ME, Price DD. Hypersensitivity to visceral and cutaneous pain in the irritable bowel syndrome. Pain. 2001;93:7-14.

102. Verne GN, Price DD, Callam CS, Zhang B, Peck J, Zhou Q. Viscerosomatic facilitation in a subset of IBS patients, an effect mediated by $\mathrm{N}$-methyl-D-aspartate receptors. The journal of pain : official journal of the American Pain Society. 2012;13:901-9.

103. Bernstein CN, Frankenstein UN, Rawsthorne P, Pitz M, Summers R, Mclntyre MC. Cortical mapping of visceral pain in patients with $\mathrm{Gl}$ disorders using functional magnetic resonance imaging. Am J Gastroenterol. 2002;97:319-27.

104. Bouin M, Plourde V, Boivin M, Riberdy M, Lupien F, Laganiere $M$, et al. Rectal distention testing in patients with irritable bowel syndrome: sensitivity, specificity, and predictive values of pain sensory thresholds. Gastroenterology. 2002;122:1771-7.

105. Spetalen S, Jacobsen MB, Vatn MH, Blomhoff S, Sandvik L. Visceral sensitivity in irritable bowel syndrome and healthy volunteers: reproducibility of the rectal barostat. Dig Dis Sci. 2004;49:1259-64.

106. Posserud I, Agerforz P, Ekman R, Bjornsson ES, Abrahamsson H, Simren M. Altered visceral perceptual and neuroendocrine response in patients with irritable bowel syndrome during mental stress. Gut. 2004;53:1102-8. 
107. Delvaux M, Beck A, Jacob J, Bouzamondo H, Weber FT, Frexinos J. Effect of asimadoline, a kappa opioid agonist, on pain induced by colonic distension in patients with irritable bowel syndrome. Aliment Pharmacol Ther. 2004;20:237-46.

108. Coffin B, Bouhassira D, Sabate JM, Barbe L, Jian R. Alteration of the spinal modulation of nociceptive processing in patients with irritable bowel syndrome. Gut. 2004;53:1465-70.

109. Wilder-Smith CH, Schindler D, Lovblad K, Redmond SM, Nirkko A. Brain functional magnetic resonance imaging of rectal pain and activation of endogenous inhibitory mechanisms in irritable bowel syndrome patient subgroups and healthy controls. Gut. 2004;53:1595-601.

110. Caldarella MP, Milano A, Laterza F, Sacco F, Balatsinou C, Lapenna D, et al. Visceral sensitivity and symptoms in patients with constipation- or diarrhea-predominant irritable bowel syndrome (IBS): effect of a low-fat intraduodenal infusion. Am J Gastroenterol. 2005;100:383-9.

111. Caldarella MP, Giamberardino MA, Sacco F, Affaitati G, Milano A, Lerza R, et al. Sensitivity disturbances in patients with irritable bowel syndrome and fibromyalgia. Am J Gastroenterol. 2006;101:2782-9.

112. Cremonini F, Houghton LA, Camilleri M, Ferber I, Fell C, Cox V, et al. Barostat testing of rectal sensation and compliance in humans: comparison of results across two centres and overall reproducibility. Neurogastroenterol Motil.2005;17:810-20.

113. Morgan V, Pickens D, Gautam S, Kessler R, Mertz H. Amitriptyline reduces rectal pain related activation of the anterior cingulate cortex in patients with irritable bowel syndrome. Gut. 2005;54:601-7.

114. Lee KJ, Kim JH, Cho SW. Gabapentin reduces rectal mechanosensitivity and increases rectal compliance in patients with diarrhoea-predominant irritable bowel syndrome. Aliment Pharmacol Ther. 2005;22: 981-8.

115. Vase L, Robinson ME, Verne GN, Price DD. Increased placebo analgesia over time in irritable bowel syndrome (IBS) patients is associated with desire and expectation but not endogenous opioid mechanisms. Pain. 2005;115:338-47.

116. Frokjaer JB, Andersen SD, Gale J, Arendt-Nielsen L, Gregersen H, Drewes AM. An experimental study of viscero-visceral hyperalgesia using an ultrasound-based multimodal sensory testing approach. Pain. 2005;119:191-200.

117. Ng C, Malcolm A, Hansen R, Kellow JE. Distension technique influences the relationship between colonic and rectal hypersensitivity in irritable bowel syndrome. Neurogastroenterol Motil.2006;18: 206-10.

118. Song GH, Venkatraman V, Ho KY, Chee MW, Yeoh KG, Wilder-Smith CH. Cortical effects of anticipation and endogenous modulation of visceral pain assessed by functional brain MRI in irritable bowel syndrome patients and healthy controls. Pain. 2006;126:79-90.

119. Moshiree B, Price DD, Robinson ME, Gaible R, Verne GN. Thermal and visceral hypersensitivity in irritable bowel syndrome patients with and without fibromyalgia. Clin J Pain. 2007;23:323-30.

120. Houghton LA, Fell C, Whorwell PJ, Jones I, Sudworth DP, Gale JD. Effect of a second-generation alpha2delta ligand (pregabalin) on visceral sensation in hypersensitive patients with irritable bowel syndrome. Gut. 2007;56:1218-25.

121. Dorn SD, Palsson OS, Thiwan SI, Kanazawa M, Clark WC, van Tilburg MA, et al. Increased colonic pain sensitivity in irritable bowel syndrome is the result of an increased tendency to report pain rather than increased neurosensory sensitivity. Gut. 2007;56:1202-9.

122. van der Veek PP, Steenvoorden M, Steens J, van der Schaar PJ, Brussee J, Masclee AA. Recto-colonic reflex is impaired in patients with irritable bowel syndrome. Neurogastroenterol Motil.2007;19:653-9.

123. Ringel Y, Drossman DA, Leserman JL, Suyenobu BY, Wilber K, et al. Effect of abuse history on pain reports and brain responses to aversive visceral stimulation: an FMRI study. Gastroenterology. 2008;134:396-404.

124. Agrawal A, Houghton LA, Lea R, Morris J, Reilly B, Whorwell PJ. Bloating and distention in irritable bowel syndrome: the role of visceral sensation. Gastroenterology. 2008;134:1882-9.

125. Piche $M$, Arsenault $M$, Poitras $P$, Rainville $P$, Bouin $M$. Widespread hypersensitivity is related to altered pain inhibition processes in irritable bowel syndrome. Pain. 2010;148:49-58.

126. Elsenbruch $S$, Rosenberger $C$, Enck $P$, Forsting $M$, Schedlowski M, Gizewski ER. Affective disturbances modulate the neural processing of visceral pain stimuli in irritable bowel syndrome: an fMRI study. Gut. 2010;59:489-95. 
127. Elsenbruch S, Rosenberger C, Bingel U, Forsting M, Schedlowski M, Gizewski ER. Patients with irritable bowel syndrome have altered emotional modulation of neural responses to visceral stimuli. Gastroenterology. 2010;139:1310-9.

128. Nozu T, Okumura T. Visceral sensation and irritable bowel syndrome; with special reference to comparison with functional abdominal pain syndrome. J Gastroenterol Hepatol. 2011;26 Suppl 3:122-7.

129. Vanhoutvin SA, Troost FJ, Kilkens TO, Lindsey PJ, Jonkers DM, Venema K, et al. Alternative procedure to shorten rectal barostat procedure for the assessment of rectal compliance and visceral perception: a feasibility study. J Gastroenterol. 2012;47:896-903.

130. Ludidi S, Conchillo JM, Keszthelyi D, Koning CJ, Vanhoutvin SA, Lindsey PJ, et al. Does meal ingestion enhance sensitivity of visceroperception assessment in irritable bowel syndrome? Neurogastroenterol Motil.2012;24:47-53, e3.

131. Ludidi S, Conchillo JM, Keszthelyi D, Van Avesaat M, Kruimel JW, Jonkers DM, et al. Rectal hypersensitivity as hallmark for irritable bowel syndrome: defining the optimal cutoff. Neurogastroenterol Motil.2012;24:729-33, e345-6.

132. Goncalves de Medeiros MT, de Oliveira RB, dos Santos AA, de Leopoldino DM, Lima MC, Nobre RA, et al. The effects of sildenafil on rectal sensitivity and tone in patients with the irritable bowel syndrome. Aliment Pharmacol Ther. 2012;35:577-86.

133. Benson S, Kattoor J, Wegner A, Hammes F, Reidick D, Grigoleit JS, et al. Acute experimental endotoxemia induces visceral hypersensitivity and altered pain evaluation in healthy humans. Pain. 2012;153:794-9.

134. Benson S, Kotsis V, Rosenberger C, Bingel U, Forsting M, Schedlowski M, et al. Behavioural and neural correlates of visceral pain sensitivity in healthy men and women: does sex matter? Eur J Pain. 2012;16:349-58.

135. Letzen JE, Craggs JG, Perlstein WM, Price DD, Robinson ME. Functional connectivity of the default mode network and its association with pain networks in irritable bowel patients assessed via lidocaine treatment. The journal of pain : official journal of the American Pain Society. 2013;14:1077-87.

136. Aniwan S, Gonlachanvit S. Effects of Chili Treatment on Gastrointestinal and Rectal Sensation in Diarrhea-predominant Irritable Bowel Syndrome: A Randomized, Double-blinded, Crossover Study. Journal of neurogastroenterology and motility. 2014;20:400-6.

137. van Wanrooij SJ, Wouters MM, Van Oudenhove L, Vanbrabant W, Mondelaers S, Kollmann P, et al. Sensitivity testing in irritable bowel syndrome with rectal capsaicin stimulations: role of TRPV1 upregulation and sensitization in visceral hypersensitivity? Am J Gastroenterol. 2014;109:99-109.

138. Melchior C, Gourcerol G, Chastan N, Verin E, Menard JF, Ducrotte P, et al. Effect of transcranial magnetic stimulation on rectal sensitivity in irritable bowel syndrome: a randomized, placebocontrolled pilot study. Colorectal Dis. 2014;16:0104-11.

139. Naliboff BD, Berman S, Suyenobu B, Labus JS, Chang L, Stains J, et al. Longitudinal change in perceptual and brain activation response to visceral stimuli in irritable bowel syndrome patients. Gastroenterology. 2006;131:352-65.

140. Rossel P, Drewes AM, Petersen P, Nielsen J, Arendt-Nielsen L. Pain produced by electric stimulation of the rectum in patients with irritable bowel syndrome: further evidence of visceral hyperalgesia. Scand J Gastroenterol. 1999;34:1001-6.

141. Brock C, Nissen TD, Gravesen FH, Frokjaer JB, Omar H, Gale J, et al. Multimodal sensory testing of the rectum and rectosigmoid: development and reproducibility of a new method. Neurogastroenterol Motil.2008;20:908-18.

142. Thoua NM, Murray CD, Winchester WJ, Roy AJ, Pitcher MC, Kamm MA, et al. Amitriptyline modifies the visceral hypersensitivity response to acute stress in the irritable bowel syndrome. Aliment Pharmacol Ther. 2009;29:552-60.

143. Arebi N, Bullas DC, Dukes GE, Gurmany S, Hicks KJ, Kamm MA, et al. Distinct neurophysiological profiles in irritable bowel syndrome. Am J Physiol Gastrointest Liver Physiol.2011;300:G1086-93.

144. U.S. Department of Health and Human Services FaDA, Center for Drug Evaluation and Research (CDER). Guidance for Industry Irritable Bowel Syndrome - Clinical Evaluation of Drugs for Treatment2012. http://wwwfdagov/downloads/Drugs/Guidances/UCM205269pdf. 
145. Lacy BE, Lembo AJ, Macdougall JE, Shiff SJ, Kurtz CB, Currie MG, et al. Responders vs clinical response: a critical analysis of data from linaclotide phase 3 clinical trials in IBS-C. Neurogastroenterol Motil.2014;26:326-33.

146. Melzack R. The McGill Pain Questionnaire: major properties and scoring methods. Pain. 1975;1:277-99.

147. Byrne M, Troy A, Bradley LA, Marchisello PJ, Geisinger KF, Van der Heide LH, et al. Cross-validation of the factor structure of the McGill Pain Questionnaire. Pain. 1982;13:193-201.

148. Mayer EA. Emerging disease model for funct. gastrointestinal disorders. Am J Med. 1999;107):12S-9S.

149. Bradburn NM, Rips LJ, Shevell SK. Answering autobiographical questions: the impact of memory and inference on surveys. Science. 1987;236:157-61.

150. Thijssen AY, Jonkers DM, Leue C, van der Veek PP, Vidakovic-Vukic M, van Rood YR, et al. Dysfunctional cognitions, anxiety and depression in irritable bowel syndrome. J Clin Gastroenterol.2010;44:e236-41.

151. Comer SD, Zacny JP, Dworkin RH, Turk DC, Bigelow GE, Foltin RW, et al. Core outcome measures for opioid abuse liability laboratory assessment studies in humans: IMMPACT recommendations. Pain. 2012;153:2315-24.

152. Stone AA, Shiffman S, Schwartz JE, Broderick JE, Hufford MR. Patient compliance with paper and electronic diaries. Control Clin Trials. 2003;24:182-99.

153. Myin-Germeys I, Oorschot M, Collip D, Lataster J, Delespaul P, van Os J. Experience sampling research in psychopathology: opening the black box of daily life. Psychol Med. 2009;39:1533-47.

154. Stone AA, Broderick JE, Schwartz JE, Shiffman S, Litcher-Kelly L, Calvanese P. Intensive momentary reporting of pain with an electronic diary: reactivity, compliance, and patient satisfaction. Pain. 2003;104:343-51.

155. Whitehead WE, Palsson OS. Is rectal pain sensitivity a biological marker for irritable bowel syndrome: psychological influences on pain perception. Gastroenterology. 1998;115:1263-71. 


\section{Supplementary information}

\section{Description of available provocation tests used to measure chronic abdominal pain}

\section{Mechanical intestinal stimulus}

In the last decades, many studies have been aiming to develop an objective biomarker for visceral pain. Different procedures using balloon distension have been designed to apply mechanical stimuli to the intestinal lumen to provoke sensations and to measure pain threshold of individuals. The induced sensation is reported and scored by the subject on a VAS, NRS or VRS. In 1973, Ritchie was the first to report increased pain sensation to distal colon balloon distension in IBS patients compared to healthy individuals. ${ }^{1}$ In 1990, Whitehead et al. used balloon distention of the rectosigmoid combined with a (somatic) thermal stimulus on the skin (hand immersion in cold water), in patients with IBS compared to patients with other GI disorders and healthy controls, and reported a significant lower tolerance for mechanical balloon distension but not for the thermal stimulus on the hand in IBS patients. Thereby proposing the rectal mechanical stimulus to be a possible specific biomarker for the visceral pain threshold in IBS. ${ }^{2}$ The increased sensitivity to urge, discomfort and pain assessed by rectal barostat balloon is referred to as visceral hypersensitivity. In case the threshold to the mechanical stimulus is lowered, it is referred to as allodynia. Hypersensitivity and allodynia to mechanical bowel distension have been observed in up to $60 \%$ of IBS patients. $^{3-5}$

In the present review the included publications have assessed visceral sensation of the rectum/colon by mechanical provocation tests in IBS patients

Reproducibility and reliability: Hammer et al. assessed reproducibility of rectal barostat in five healthy individuals and concluded that the day-to-day variability was sufficiently small to allow valid comparisons to be made. ${ }^{6}$ Spetalen et al. described an 'acceptable reproducibility' of rectal barostat measurements assessed in a group 33 IBS patients and healthy controls, reporting a repeatability coefficient of 2.4 and an interclass correlation coefficient of 0.76-0.93 in healthy controls and 0.53-0.88 in IBS patients. The pooled coefficient of variation (CV) ranged from $10-24 \%$ and $11-49 \%$, respectively. ${ }^{7}$ Cremonini et al. described overall reproducibility of a rectal barostat test in 34 healthy individuals as 'good', showing an inter-individual CV for pain ranged between 31-37\%, while the intra-individual CV for pain was $23 \% .^{8}$ Based on these data the rectal barostat is currently regarded as a reproducible and reliable measure for visceral sensitivity in patients with IBS and healthy individuals.

To answer the question whether rectal sensitivity is comparable to or represents colonic sensitivity $\mathrm{Ng}$ et al. correlated barostat distensions VAS scores of rectum and descending colon in 20 IBS patients and 12 healthy controls, and observed a significant 
correlation of $r=0.60$ for pain and concluded that the rectum serves as a legitimate representative for the colon with respect to visceral hypersensitivity. ${ }^{9}$

Sensitivity and specificity: Bouin et al. reported values for sensitivity and specificity of $95.5 \%$ and $71.8 \%$ at $40 \mathrm{mmHg}$ balloon pressure, respectively. The positive and negative predictive values at the same balloon pressure were $85 \%$ and $90 \%$, respectively. ${ }^{10}$ More recently, Ludidi et al. calculated the optimal cut-off value to detect visceral hypersensitivity in IBS. The optimal cut-off occurred at a balloon pressure of $26 \mathrm{mmHg}$ with a VAS of $20 \mathrm{~mm}$ resulting in $63.5 \%$ hypersensitive and $11 \%$ allodynic IBS patients. The sensitivity, specificity and positive predictive value at this cut-off point were $63 \%$, $90 \%$ and $96 \%$, respectively. ${ }^{3}$

Correlation with symptom severity: The correlation of pain threshold measured by mechanical provocation tests and symptom severity assessed by a variety of questionnaires was poor.

\section{Nutrient or chemical provocation test}

A frequent trigger of abdominal pain in IBS patients is food intake. ${ }^{11}$ Rectal and colonic perception threshold in IBS patients have shown to decrease after intraduodenal infusion of fat. ${ }^{12,13}$ Moreover liquid meals, with a more pronounced effect of fatty compounds compared to carbohydrate, have shown to enhance visceral sensitivity in the same population. ${ }^{14-16}$ Therefore visceral provocation tests are mostly conducted in a fasting condition. But, assessing postprandial visceral sensitivity in a standardized condition could augment the understanding of visceral perception and add a further dimension to the visceral sensitivity assessment of IBS patients. ${ }^{17}$

Several research groups have used specific chemical stimuli to study pain mechanisms. Frokjaer et al. used an acid provocation test in combination with mechanical and thermal stimuli to assess viscero-visceral hyperalgesia in 12 healthy controls. ${ }^{18}$ Furthermore, capsaicin, the red pepper ingredient responsible for the heat sensation, was used to induce abdominal pain or discomfort in healthy controls. ${ }^{19-22}$ Data on validity of these methods are lacking, so further research is needed.

\section{Thermal or electric intestinal stimulus}

Thermal or electrical stimuli in the intestine to provoke sensations and measure visceral sensitivity are alternatives to the balloon distension. ${ }^{18,23-26}$ Brock et al. combined mechanical, thermal and electric stimuli in the rectosigmoid, tested reproducibility and reported separate pain detection threshold intra-class correlation coefficients (ICC) for the different stimuli: 0.9 for repeated electrical stimuli, 0.7 for heat, 0.9 for cold and for the mechanical stimuli (no difference between days was found), thereby showing an high reproducibility. ${ }^{26}$ In contrast to bowel distension, thermal and electrical stimuli do not mimic physiological condition of the intestine. 


\section{Thermal or electric stimuli on the skin combined with visceral stimuli}

The discussion is still on-going whether IBS patients show more generalised hypersensitivity and thereby suggesting the presence of a general pain disorders in these patients with involvement of central hyperalgesic mechanisms, instead of isolated visceral hypersensitivity.

Several studies combined provocation tests of the intestine in IBS patients with thermal or electric stimuli on the skin to broadly assess sensitivity. In 1990, Whitehead et al., demonstrated that IBS patients had an increased response to rectal balloon distension, but not to thermal hand skin stimuli (cold water). ${ }^{2}$ This was confirmed by Rössel et al. using cutaneous electrical stimuli. ${ }^{25}$ Caldarella et al. used rectal distension and electric skin stimuli to compare IBS to fibromyalgia (FM) patients, and observed increased visceral sensitivity in IBS and increased somatic sensitivity in FM patients, indicating that different mechanisms are involved in pain modulation in these patient groups. ${ }^{27}$ Moshiree et al. employed rectal distension and thermal skin stimuli in similar patient groups. They point to an increased response to skin and visceral stimuli in both patient groups. The IBS group exhibited higher pain ratings to rectal distension but lower pain ratings to thermal skin stimuli compared to the IBS + FM group. Thus, the regions of primary and secondary hyperalgesia depend on the origin of the primary pain complaint. $^{28}$ Verne et al. have come to opposing conclusions by demonstrating both visceral hypersensitivity to mechanical distension in the rectum and cutaneous allodynia/hyperalgesia to thermal stimuli in IBS patients. The cutaneous hyperalgesia was more pronounced in the lower versus the upper extremities, suggesting a rostralcaudal distribution of increased pain sensitivity. ${ }^{29}$ In a recent study, the same researchers observed that repetitive visceral distension stimuli and repetitive cutaneous thermal stimuli enhance bidirectional mechanisms of secondary hyperalgesia in a subgroup with visceral hypersensitivity. This enhancement was not observed in healthy controls or normosensitive IBS patients. ${ }^{30}$ Piche et al. reported a relationship between altered pain inhibition processes and widespread hypersensitivity in IBS. ${ }^{31}$ Stabell et al. demonstrated lower thermal pain thresholds, for cold and heat, on the upper extremities in a large group of subjects reporting IBS symptoms, when compared to healthy controls. ${ }^{32}$

In conclusion, there are contradicting findings in the current literature on this topic. However, when considering all available evidence, a subgroup of IBS patients may be affected not only by visceral, but by a general hypersensitivity to pain.

\section{Colonoscopy as provocation test}

Kim et al. assessed colonoscopy associated pain, assuming IBS patients to experience more pain during colonoscopy compared to other individuals. They demonstrated an increased perception of pain during colonoscopy in IBS patients. ${ }^{33}$ This provocation method has not been reproduced by other research groups. 


\section{References}

1. Ritchie J. Pain from distension of the pelvic colon by inflating a balloon in the irritable colon syndrome. Gut. 1973;14:125-32.

2. Whitehead WE, Holtkotter B, Enck P, Hoelzl R, Holmes KD, Anthony J, et al. Tolerance for rectosigmoid distention in irritable bowel syndrome. Gastroenterology. 1990;98:1187-92.

3. Ludidi S, Conchillo JM, Keszthelyi D, Van Avesaat M, Kruimel JW, Jonkers DM, et al. Rectal hypersensitivity as hallmark for irritable bowel syndrome: defining the optimal cutoff. Neurogastroenterol Motil. 2012;24:729-33, e345-6.

4. Kuiken SD, Lindeboom R, Tytgat GN, Boeckxstaens GE. Relationship between symptoms and hypersensitivity to rectal distension in patients with irritable bowel syndrome. Aliment Pharmacol Ther.2005;22:157-64.

5. Ludidi S, Mujagic Z, Jonkers D, Keszthelyi D, Hesselink M, Kruimel J, et al. Markers for visceral hypersensitivity in patients with irritable bowel syndrome. Neurogastroenterol Motil. 2014;26:1104-11.

6. Hammer HF, Phillips SF, Camilleri M, Hanson RB. Rectal tone, distensibility, and perception: reproducibility and response to different distensions. Am J Physiol. 1998;274:G584-90.

7. Spetalen S, Jacobsen MB, Vatn $\mathrm{MH}$, Blomhoff $\mathrm{S}$, Sandvik L. Visceral sensitivity in irritable bowel syndrome and healthy volunteers: reproducibility of the rectal barostat. Dig Dis Sci. 2004;49:1259-64.

8. Cremonini F, Houghton LA, Camilleri M, Ferber I, Fell C, Cox V, et al. Barostat testing of rectal sensation and compliance in humans: comparison of results across two centres and overall reproducibility. Neurogastroenterol Motil. 2005;17:810-20.

9. $\mathrm{Ng} \mathrm{C}$, Malcolm A, Hansen R, Kellow JE. Distension technique influences the relationship between colonic and rectal hypersensitivity in irritable bowel syndrome. Neurogastroenterol Motil. 2006;18: 206-10.

10. Bouin $M$, Plourde V, Boivin M, Riberdy $M$, Lupien $F$, Laganiere $M$, et al. Rectal distention testing in patients with irritable bowel syndrome: sensitivity, specificity, and predictive values of pain sensory thresholds. Gastroenterology. 2002;122:1771-7.

11. Simren M, Mansson A, Langkilde AM, Svedlund J, Abrahamsson H, Bengtsson U, et al. Food-related gastrointestinal symptoms in the irritable bowel syndrome. Digestion. 2001;63:108-15.

12. Caldarella MP, Milano A, Laterza F, Sacco F, Balatsinou C, Lapenna D, et al. Visceral sensitivity and symptoms in patients with constipation- or diarrhea-predominant irritable bowel syndrome (IBS): effect of a low-fat intraduodenal infusion. Am J Gastroenterol. 2005;100:383-9.

13. Simren $M$, Abrahamsson $H$, Bjornsson ES. An exaggerated sensory component of the gastrocolonic response in patients with irritable bowel syndrome. Gut. 2001;48:20-7.

14. Ludidi S, Conchillo JM, Keszthelyi D, Koning CJ, Vanhoutvin SA, Lindsey PJ, et al. Does meal ingestion enhance sensitivity of visceroperception assessment in irritable bowel syndrome? Neurogastroenterol Motil. 2012;24:47-53, e3.

15. Simren M, Agerforz P, Bjornsson ES, Abrahamsson H. Nutrient-dependent enhancement of rectal sensitivity in irritable bowel syndrome (IBS). Neurogastroenterol Motil. 2007;19:20-9.

16. van der Veek PP, Steenvoorden M, Steens J, van der Schaar PJ, Brussee J, Masclee AA. Recto-colonic reflex is impaired in patients with irritable bowel syndrome. Neurogastroenterol Motil. 2007;19:653-9.

17. Keszthelyi D, Troost FJ, Masclee AA. Irritable bowel syndrome: methods, mechanisms, and pathophysiology. Methods to assess visceral hypersensitivity in irritable bowel syndrome. Am J Physiol Gastrointest Liver Physiol. 2012;303:G141-54.

18. Frokjaer JB, Andersen SD, Gale J, Arendt-Nielsen L, Gregersen H, Drewes AM. An experimental study of viscero-visceral hyperalgesia using an ultrasound-based multimodal sensory testing approach. Pain. 2005;119:191-200.

19. Cao Y, Wilder-Smith CH, Li XH, Wong RK, Hammer J, Ho KY. Characterization of a reproducible gastric pain model using oral capsaicin titration in healthy volunteers. Neurogastroenterol Motil. 2011;23:e261-70.

20. Hammer J, Vogelsang H. Characterization of sensations induced by capsaicin in the upper gastrointestinal tract. Neurogastroenterol Motil. 2007;19:279-87. 
21. van Wanrooij SJ, Wouters MM, Van Oudenhove L, Vanbrabant W, Mondelaers S, Kollmann P, et al. Sensitivity testing in irritable bowel syndrome with rectal capsaicin stimulations: role of TRPV1 upregulation and sensitization in visceral hypersensitivity? Am J Gastroenterol. 2014;109:99-109.

22. Aniwan S, Gonlachanvit S. Effects of Chili Treatment on Gastrointestinal and Rectal Sensation in Diarrhea-predominant Irritable Bowel Syndrome: A Randomized, Double-blinded, Crossover Study. J Neurogastroenterol Motil.2014;20:400-6.

23. Arebi N, Bullas DC, Dukes GE, Gurmany S, Hicks KJ, Kamm MA, et al. Distinct neurophysiological profiles in irritable bowel syndrome. Am J Physiol Gastrointest Liver Physiol. 2011;300:G1086-93.

24. Thoua NM, Murray CD, Winchester WJ, Roy AJ, Pitcher MC, Kamm MA, et al. Amitriptyline modifies the visceral hypersensitivity response to acute stress in the irritable bowel syndrome. Aliment Pharmacol Ther. 2009;29:552-60.

25. Rossel P, Drewes AM, Petersen P, Nielsen J, Arendt-Nielsen L. Pain produced by electric stimulation of the rectum in patients with irritable bowel syndrome: further evidence of visceral hyperalgesia. Scan J Gastroent. 1999;34:1001-6.

26. Brock C, Nissen TD, Gravesen FH, Frokjaer JB, Omar H, Gale J, et al. Multimodal sensory testing of the rectum and rectosigmoid: development and reproducibility of a new method. Neurogastroenterol Motil. 2008;20:908-18.

27. Stabell N, Stubhaug A, Flaegstad T, Nielsen CS. Increased pain sensitivity among adults reporting irritable bowel syndrome symptoms in a large population-based study. Pain. 2013;154:385-92.

28. Caldarella MP, Giamberardino MA, Sacco F, Affaitati G, Milano A, Lerza R, et al. Sensitivity disturbances in patients with irritable bowel syndrome and fibromyalgia. Am J Gastroenterol. 2006;101:2782-9.

29. Moshiree B, Price DD, Robinson ME, Gaible R, Verne GN. Thermal and visceral hypersensitivity in irritable bowel syndrome patients with and without fibromyalgia. Clin J Pain. 2007;23:323-30.

30. Verne GN, Robinson ME, Price DD. Hypersensitivity to visceral and cutaneous pain in the irritable bowel syndrome. Pain. 2001;93:7-14

31. Verne GN, Price DD, Callam CS, Zhang B, Peck J, Zhou Q. Viscerosomatic facilitation in a subset of IBS patients, an effect mediated by N-methyl-D-aspartate receptors. J Pain. 2012;13:901-9.

32. Piche $M$, Arsenault $M$, Poitras $P$, Rainville $P$, Bouin $M$. Widespread hypersensitivity is related to altered pain inhibition processes in irritable bowel syndrome. Pain. 2010;148:49-58.

33. Kim ES, Cheon JH, Park JJ, Moon CM, Hong SP, Kim TI, et al. Colonoscopy as an adjunctive method for the diagnosis of irritable bowel syndrome: focus on pain perception. J Gastroenterol Hepatol.2010;25:1232-8. 
Measurement of abdominal pain in IBS 


\section{Chapter}

\section{ESM - a new digital tool for symptom assessment in IBS}

The experience sampling method - a new digital tool for momentary symptom assessment in IBS: an exploratory study

Zlatan Mujagic, Carsten Leue, Lisa Vork, Richel Lousberg, Daisy MAE Jonkers, Daniel Keszthelyi, Martine AM Hesselink, Thirza JC van Schagen, Jim van Os, Ad AM Masclee, Joanna W Kruimel

Neurogastroenterology and Motility 2015;27:1295-1302 


\section{Abstract}

\section{Background}

Retrospective questionnaires are frequently used for symptom assessment in Irritable Bowel Syndrome (IBS) patients, but are influenced by recall bias and circumstantial and psychological factors. These limitations may be overcome by random, repeated, momentary assessment during the day, using electronic Experience Sampling Methodology (ESM). Therefore, we compared symptom assessment by ESM to retrospective paper questionnaires in IBS patients.

\section{Methods}

Twenty-six IBS patients (Rome III) were included, of which 16 were diagnosed with panic disorder (DSM-IV-TR). Patients scored symptoms using end-of-day diaries during 14 days and the gastrointestinal symptom rating scale (GSRS) once. ESM was used on 7 consecutive days during the same time period.

\section{Key results}

End-of-day diary abdominal pain scores were 0.4 (SE 0.1, $\mathrm{p}<0.001$ ) point higher (on a 1-to-5-point scale) compared to corresponding ESM mean-scores in IBS patients. The difference was even more pronounced for upper abdominal pain scores assessed by the GSRS (4.77 \pm 1.50$)$ compared to ESM mean-scores (2.44 $\pm 1.30, p<0.001)$, both on 1-to-7point scale. For flatulence, comparable results were found. Nausea and belching scores showed small, but significant differences between end-of-day diary and ESM. All tested symptoms were scored higher on GSRS compared to ESM mean-scores $(p<0.01)$. Affective comorbidity did not influence differences in pain reporting between methods.

\section{Conclusion}

IBS patients report higher scores for abdominal pain in retrospective questionnaires compared to ESM, with a tendency to report peak rather than average pain scores. ESM can provide more insight in symptom course and potential triggers, and may lead to a better understanding of IBS symptomatology. 


\section{Introduction}

A major hurdle for the development of new therapies in Irritable Bowel Syndrome (IBS) is the current lack of reliable instruments to measure symptoms over time and quantify therapy response. At present, retrospective self-reported paper questionnaires based on daily (i.e. by end-of-day diaries) or weekly symptom monitoring (i.e. by end-of-week questionnaires) are widely used as the standard method to assess symptoms in IBS patients. ${ }^{1-4}$ The Food and Drugs Administration (FDA) recommends a 7-day mean of an 'end-of-day 11-point Numeric Rating Scale' as a primary endpoint for clinical trials assessing abdominal pain in IBS. ${ }^{5}$ In a recently published study, Lackner et al. demonstrated that (electronic) end-of-day diaries may more accurately quantify GI symptoms in IBS patients compared to weekly symptom monitoring, in particular for average abdominal pain over a predefined period of time. ${ }^{4}$ However, it should be taken into account that all retrospective questionnaires (e.g. end-of-day or end-of-week symptom assessment) are based on autobiographical memory, thereby leading to recall bias, representing a reconstruction of specific moments, rather than a reliable reflection of symptoms over a time period. ${ }^{6}$ Memory of pain can be influenced by contextual, as well as by emotional and cognitive factors. This can affect perception and interpretation of symptoms, especially pain, and may result in state-congruent and ecological recall bias in retrospective questionnaires. ${ }^{7-9}$ Affective comorbidity such as anxiety disorders, are prevalent in IBS patients and the presence of psychological comorbidity may contribute to a distorted perception and memory of Gl symptoms as well. ${ }^{10,11}$ Furthermore, the inability to record temporal dynamics of symptoms renders retrospective questionnaires less suitable for measurement of symptoms fluctuating over time. Finally, it has been shown that paper diaries are subject to low compliance rates, as low as approximately $10 \%$, due to backfilling of questionnaires. ${ }^{12}$

The Experience Sampling Method (ESM), ${ }^{13}$ also referred to as Ecological Momentary Assessment $(E M A){ }^{14}$ may overcome the significant limitations of retrospective questionnaires. ${ }^{15}$ This assessment method is characterized by the following criteria: data are collected repeatedly and randomly during the day, for several days, using a digital device, in the subject's natural environment, with a focus on the subject's in-themoment (i.e. momentary) physical and mental state and behaviour. Digital momentary assessment reduces recall and ecological bias, may increase compliance rates and can take into account emotional, cognitive and contextual factors impacting IBS symptom reporting in general and abdominal pain in particular. One previous study by Weinland and colleagues used electronic momentary assessment in a group of 58 IBS patients to describe differences in symptom episodes between IBS subtypes. As secondary outcome they reported that abdominal pain scores were significantly higher in the traditional paper end-of-day diaries when compared to the electronic momentary assessment. ${ }^{16}$ More research is needed to confirm their observation for abdominal pain, but also to compare ESM to current standards (i.e. daily and weekly retrospective 
symptom reporting) with regard to other Gl symptoms, as well as to investigate possible patient characteristics, such as anxiety, that may affect pain memory and therefore the differences between momentary and retrospective symptom assessment.

The aims of the present study were to explore possible advantages of electronic ESM over paper retrospective questionnaires for GI symptom measurement in IBS patients, by i) comparing abdominal pain and other GI symptom scores assessed by a paper based end-of-day diary to corresponding scores assessed by ESM, by ii) comparing the GSRS, which includes a recall period of one week, to ESM, and by iii) investigating patient characteristics, that may affect memory of pain and may therefore contribute to a possible difference in scores between the momentary and retrospective symptom assessment.

We hypothesized that a) IBS patients will report higher levels of abdominal pain, and possibly also other GI symptoms, in end-of-day diaries and the GSRS compared to ESM average scores, and $b$ ) that the presence of a comorbid panic disorder, more severe mental symptoms and negative cognitions will influence this difference in symptom reporting between the methods.

\section{Materials and methods}

The present study is part of a larger cohort study on the phenotypical and genotypical characterization of IBS (Maastricht IBS cohort) and of a 'Randomized Controlled Trial of Escitalopram versus Placebo for Patients with Irritable Bowel Syndrome and Panic Disorder', of which the baseline data (i.e. before start of any intervention) were used for the present analysis. Study protocols were approved by the Maastricht University Medical Center+ (MUMC+) Ethics Committee, were in compliance with the revised Declaration of Helsinki (64th WMA General Assembly, Fortaleza, Brazil, 2013) and were registered in the US National Library of Medicine (http://www.clinicaltrials.gov, NCT00775060 and NCT01551225, respectively).

\section{Study participants}

Subjects between 18 and 75 years of age with IBS, diagnosed by a gastroenterologist using the Rome III criteria, $^{17}$ were recruited via the MUMC+ GastroenterologyHepatology outpatient clinic, which is a secondary and tertiary referral centre for patients with functional GI disorders. For the diagnosis of IBS, GI endoscopy with biopsies, abdominal imaging by ultrasonography or CT scan, and/or blood, breath and faecal analysis were performed to exclude organic disease, when indicated by the physician. Patients with a history of abdominal surgery were excluded, with the 
exception of appendectomy, laparoscopic cholecystectomy or hysterectomy. The presence of co-morbid panic disorder was diagnosed by an experienced psychiatrist based on the DSM-IV-TR criteria, by using the Mini-International-NeuropsychiatricInterview (M.I.N.I.). ${ }^{18}$ All subjects gave written informed consent prior to participation.

\section{Paper questionnaires and ESM}

Participants completed questionnaires on demographics, lifestyle factors and use of medication. Furthermore, they completed scales indexing psychopathology and negative cognitions. The Hospital Anxiety and Depression Scale (HADS), consisting of 14 items clustered into two subscales, i.e. anxiety and depression scale, with a score of 0-21 per subscale, and a recall period of one week. Higher scores indicate higher symptom burden. ${ }^{19,20}$ The Cognitive Scale for Functional Bowel Disorders (CS-FBD), consisting of 31 items, scored on a 7-point Likert scale, and a total score of 31-217, with a recall period of one month, was used to assess dysfunctional cognitions related to symptoms in FBD. A higher score indicates more dysfunctional cognitions. ${ }^{21}$

GI symptoms were assessed over 14 consecutive days using an end-of-day diary. Complaints of abdominal discomfort, abdominal pain, nausea, bloating, belching, flatulence, diarrhoea, constipation and overall symptom burden were scored on 5-point Likert scales. $^{2,3,22}$

Electronic $\mathrm{ESM}^{13}$ was used to assess real-time GI symptoms (e.g. abdominal pain, heartburn, nausea, belching, bloating, flatulence) in daily life, as well as momentary physical and mental experiences, activities and contextual aspects, during 7 consecutive days of the same time period. ESM was started at a random day during the first week of the 14 day end-of-day diary period. Subjects carried a digital device, the PsyMate (comparable to a mobile phone). ${ }^{23}$ The device gives an auditory signal (a "beep") at ten random time points per day. Participants were instructed to complete 60 short questions on the device following every signal, using a 7-point Likert scale. The completion of the question set took 4-5 minutes on each occasion. The same set of questions was repeated in the same order at every beep. As subjects are usually not able to respond after every beep (for example because of driving a car), completion of $\geq 6$ of the 10 beep-questionnaires per day was considered as being compliant. ${ }^{24}$

In addition, participants were instructed to complete the Gastrointestinal Symptom Rating Scale (GSRS), consisting of 15 items, scored on a 7-point Likert scale, with a recall period of one week, during the same time period. ${ }^{25}$

Individual questions of both retrospective methods were compared to individual questions of ESM. Only GI symptoms assessed by ESM that corresponded to questions in the end-of-day diary and/or GSRS were included in the current analyses. The phrasing of the questions was similar, apart from the time frame explanation, and for the end-of-day diary with regard to the used scale, i.e. a 1-to-5 point scale for the endof-day diary compared to a 1-to-7 point scale for the ESM and GSRS. 


\section{Data and statistical analysis}

4D software was used to program PsyMate devices and extract the data. Statistical analyses were performed using IBM SPSS Statistics version 22.0 (IBM Statistics for Windows, Armonk, New York).

In order to check if completing the ESM questionnaires at multiple time points each day influenced the scores of the end-of-day diary, we compared the end-of-day diary scores (i.e. 7-day mean of each symptom) of the 7 days during which participants also carried the PsyMate device to the 7 days during which subjects only completed the end-of-day diary, using a paired sample t-test.

ESM data are based on a multi-level structure, including the variation between subjects and repeated measures within subjects. Associations between Gl symptom scores of the end-of-day diary and corresponding ESM scores were tested, using linear mixed models, based on a random intercept and an autoregressive (AR1) covariate structure. Currently the end-of-day diary is the standard method and was therefore used as dependent variable in the analysis. For each participant, ESM day-mean scores of individual symptoms were used as predictor, to test whether average symptom scores of the day reflect the end-of-day-diary scores.

Scoring scales of the end-of-day diaries and ESM were harmonized by rescaling the ESM data from a 7-point to a 5-point scale, i.e. a score of 1 was 1, 2 or 3 was rescaled to 2, 4 to 3,5 or 6 to 4 and 7 to 5 . In order to assess whether the rescaling affected the reliability of GI symptom related questions in ESM, a Cronbach's $\alpha$ was calculated for the original and rescaled data.

To quantify the difference of scores between the two methods, ESM data were subtracted from corresponding end-of-day diary scores, resulting in a delta score, which was statistically tested using the same multi-level analysis, adjusting for repeated measures. In like manner, the differences between end-of-day diary scores and ESM maximum scores of the day were tested.

In order to investigate if certain patient characteristics lead to a different retrospective perception of abdominal pain, possible predictors of a larger delta-score (i.e. the differences in abdominal pain reporting between ESM day-mean scores and the end-ofday diary scores) were tested: i.e. sex, age, presence of co-morbid panic disorder, psychological symptoms (HADS) and negative cognitions (CS-FBD). To further test the hypothesis that the presence of panic disorder in patients may influence GI symptom reporting, linear mixed models were used to investigate whether there was a difference in symptom score levels between IBS patients with and without a diagnosis of panic disorder, within ESM or end-of-day diary data.

Differences between GI symptom scores assessed by the GSRS and ESM could only be analysed on subject level, by paired sample t-test, due to lack of intra-subject variation in the GSRS scores (only one measurement per subject). Subject-means (i.e. means over 
7 days) of non-rescaled ESM data were calculated and compared to the corresponding GSRS scores (both ESM and GSRS use a 7-point scale).

\section{Results}

Twenty-six IBS patients were included in the analysis. Female gender was predominant $(69.2 \%)$, mean age was $41.8 \pm 15.5$ years and mean $B M I$ was $25.5 \pm 5.2 \mathrm{~kg} / \mathrm{m}^{2}$. IBS subtypes were defined based on bowel habits according to the ROME III criteria: 14 subjects were classified as diarrhoea predominant (IBS-D), 4 as constipation predominant (IBS-C), 7 as mixed (IBS-M) and 1 as undefined subtype (IBS-U). Sixteen patients were diagnosed with co-morbid panic disorder. Mean scores for CS-FBD, measuring negative cognitions related to GI symptoms, and the HADS subscales for depression and anxiety were $112 \pm 44,4.2 \pm 3.7$ and $7.4 \pm 4.4$, respectively.

The scores of the 14 day paper end-of-day diary did not differ significantly between the 7 days on which also ESM was used compared to 7 days without ESM (Supplementary Table S9.1), indicating that carrying the PsyMate device did not influence the end-ofday diary scores.

All subjects reported some level of abdominal pain during the test period, and the rate of symptomatic days, on which abdominal pain was present, i.e. end-of-day diary score or at least once daily ESM score of $\geq 2$, was $84 \%$ and $86 \%$, respectively. The compliance rate was $76.8 \%$ for ESM, i.e. out of the total number of days on which ESM was used by all study participants, on $76.8 \%$ of the days at least 6 beep-questionnaires had been completed. Not completed beep-questionnaires were considered as missing values in the analyses.

To compare end-of-day diary to ESM scores, the scales of the two methods were harmonized (i.e. ESM from a 7-point to a 5-point scale). A Cronbach's $\alpha$ was calculated for the original and rescaled ESM data; $\alpha=0.647$ versus 0.648 , respectively, demonstrating that the rescaling did not influence the data strongly.

\section{End-of-day diary compared to ESM scores}

There was a significant association between end-of-day diary scores and corresponding ESM day-mean scores for all GI symptoms analysed (Table 9.1), demonstrating that both methods measure the same construct, with estimates (indicating the direction and strength of the association) ranging between 0.38 (SE 0.10) and 1.08 (SE 0.06), p<0.001. 
Table 9.1 Association between end-of-day diary scores and corresponding ESM day-mean scores, using linear mixed models (random intercept and AR1 covariate structure) and corrected for repeated measures: the dependent variable is the end-of-day diary score and the predictor is the corresponding ESM item. Estimate indicates the direction and strength of the association.

\begin{tabular}{lcccc}
\hline Gl symptom & Estimate & Std. Error & $p$-value & $95 \% \mathrm{Cl}$ \\
\hline Abdominal pain & 0.38 & 0.10 & $<0.001$ & $0.19 ; 0.59$ \\
Nausea & 0.93 & 0.07 & $<0.001$ & $0.80 ; 1.07$ \\
Belching & 1.08 & 0.06 & $<0.001$ & $0.95 ; 1.20$ \\
Bloating & 0.75 & 0.08 & $<0.001$ & $0.59 ; 0.90$ \\
Flatulence & 0.49 & 0.08 & $<0.001$ & $0.33 ; 0.66$ \\
\hline
\end{tabular}

In order to calculate possible differences in scores between both methods, the delta score was tested using the same multi-level analysis (Table 9.2). The estimate of the intercept represents the difference between the scores of the two methods. On a 1-to5-point scale, abdominal pain and flatulence scores were 0.42 (SE 0.11) and 0.41 (SE $0.06)$ point higher, respectively $(p<0.001)$, for the end-of-day diary compared to the corresponding ESM scores. Small, though statistically significant differences were also observed for nausea (0.07 (SE 0.03), $p=0.032$ ), and belching (0.13 (SE 0.03), $p<0.001$ ), but the difference was not significant for bloating. In contrast, ESM day-maximum scores did not differ when compared to paper end-of-day diary scores for abdominal pain (Figure 9.1A), but were significantly higher for bloating (Figure 9.1B), nausea, belching and flatulence (Supplementary Figure S9.1).

Table 9.2 Difference between end-of-day diary scores and corresponding ESM scores, i.e. the delta score (as dependent), tested using linear mixed models (random intercept and AR1 covariate structure) and corrected for repeated measures. Estimate represents the difference between the scores of the two methods on a 1-to-5-point scale. A positive estimate indicates a higher score in the end-of-day diary when compared to ESM.

\begin{tabular}{lccrc}
\hline Gl symptom delta score & Estimate intercept & Std. Error & \multicolumn{1}{c}{ p-value } & \multicolumn{1}{c}{$95 \% \mathrm{Cl}$} \\
\hline Abdominal pain & 0.42 & 0.11 & $<0.001$ & $0.19 ; 0.65$ \\
Nausea & 0.07 & 0.03 & 0.032 & $0.01 ; 0.13$ \\
Belching & 0.13 & 0.03 & $<0.001$ & $0.06 ; 0.20$ \\
Bloating & 0.09 & 0.06 & 0.113 & $-0.02 ; 0.20$ \\
Flatulence & 0.41 & 0.06 & $<0.001$ & $0.30 ; 0.54$ \\
\hline
\end{tabular}

\section{Predictors of differences in abdominal pain reporting between ESM and end-of-day diary}

In order to identify possible predictors for the difference in abdominal pain reporting between the two methods, delta scores were used as dependent variable in the multilevel analysis. Only the interaction between the presence of panic disorder and male sex resulted in a statistically significant larger difference between end-of-day diary and the corresponding ESM pain score (estimate 1.88 (SE 0.87), $p=0.041$ ). When taking into account multiple testing, this result was no longer statistically significant. No 
significant effect could be demonstrated for age, presence of panic disorder in general, higher anxiety and depression scores (HADS), and negative cognitions (CS-FBD).

However, within each method (i.e. ESM and end-of-day diary), IBS patients with panic disorder reported higher abdominal pain scores compared to those without; mean difference between groups was $1.1 \pm 0.2$ point $(p<0.01)$ on a 1-to-7-point scale in $E S M$, and $0.8 \pm 0.4$ point $(p<0.01)$ on a 1 -to-5-point scale in the end-of-day diary. This was also found for bloating (ESM: $1.6 \pm 0.2, p<0.01$, and end-of-day diary: $1.2 \pm 0.2, p<0.01$ ), while there were no significant differences for nausea, belching and flatulence between scores of patients with and without the diagnosis of panic disorder.

A Abdominal pain

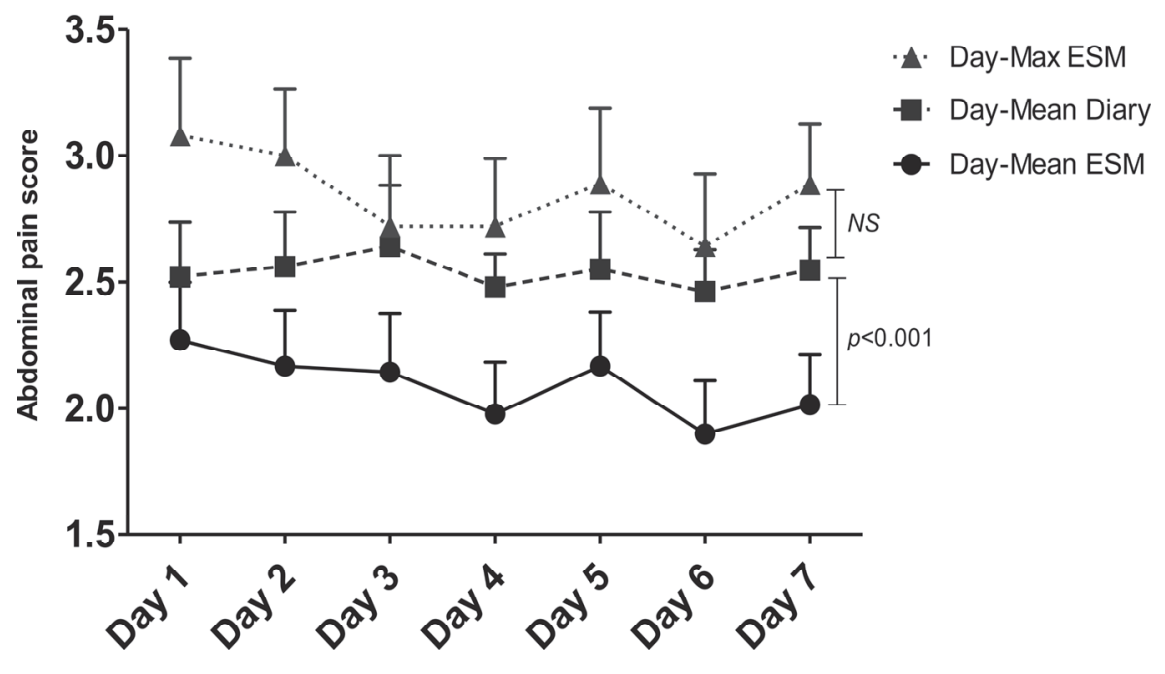

Figures 9.1 (A) End-of-day diary scores compared to ESM day-mean and day-maximum scores, for abdominal pain. Differences tested for both ESM day-mean and ESM day-max scores vs. end-of-diary scores, using linear mixed models. 


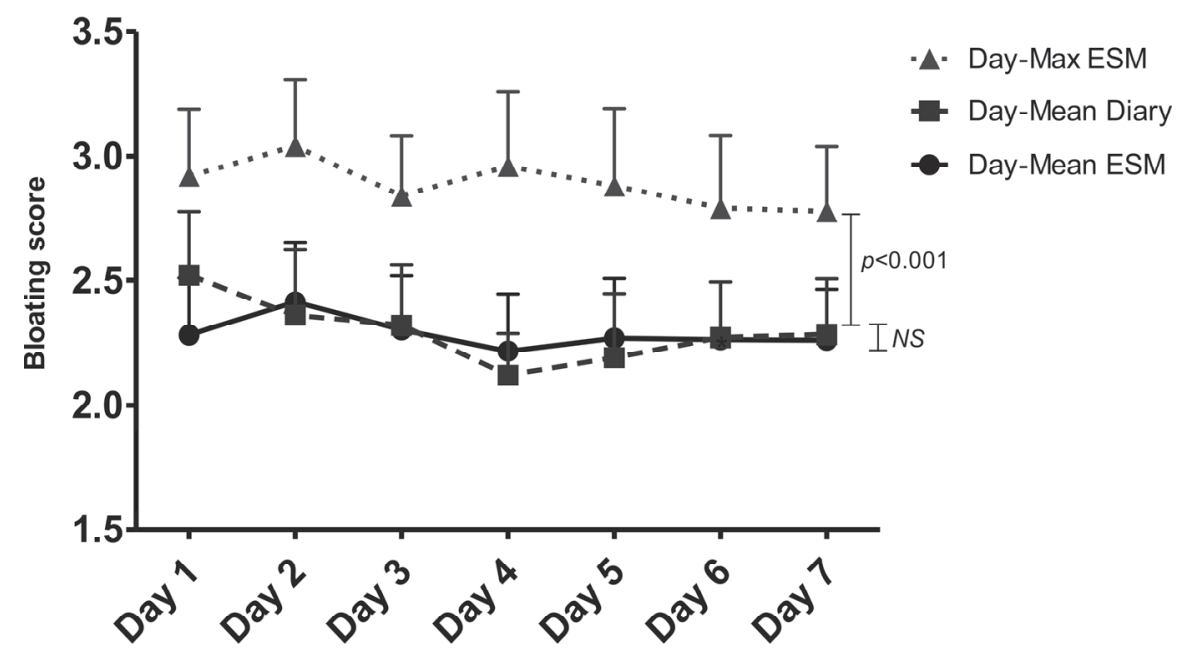

Figures 9.1 (B) End-of-day diary scores compared to ESM day-mean and day-maximum scores, for bloating. Differences tested for both ESM day-mean and ESM day-max scores vs. endof-diary scores, using linear mixed models.

\section{GSRS compared to corresponding ESM scores}

Symptom scores of the GSRS, reflecting a one-week period, were also compared to corresponding ESM 7-day-mean scores per subject. For all symptoms (i.e. upper abdominal pain, nausea, heartburn, bloating and flatulence), GSRS scores were significantly higher compared to ESM scores $(p<0.01)$, with the most pronounced difference noted for pain and flatulence (Table 9.3).

Table 9.3 GSRS scores compared to corresponding ESM symptom scores. Means calculated on subject level, SD indicates variation between subjects. Scores were not rescaled (original 1-to-7-point scale in GSRS and ESM). ${ }^{a} p<0.01,{ }^{b} p<0.001$

\begin{tabular}{|c|c|c|}
\hline $\begin{array}{l}\text { Scale } \\
\text { Gl symptom (mean } \pm \text { SD) }\end{array}$ & $\begin{array}{c}\text { GSRS } \\
\text { IBS patients }(n=26) \\
\end{array}$ & $\begin{array}{c}\text { ESM } \\
\text { IBS patients }(n=26) \\
\end{array}$ \\
\hline \multicolumn{3}{|l|}{7 point scale: } \\
\hline Upper abdominal pain & $4.77 \pm 1.50$ & $2.44 \pm 1.30^{b}$ \\
\hline Heartburn & $2.54 \pm 1.61$ & $1.11 \pm 0.28^{b}$ \\
\hline Nausea & $2.69 \pm 1.69$ & $1.45 \pm 0.88^{\mathrm{a}}$ \\
\hline Bloating & $4.35 \pm 1.81$ & $2.94 \pm 1.67^{\mathrm{a}}$ \\
\hline Flatulence & $4.69 \pm 1.38$ & $2.13 \pm 1.38^{b}$ \\
\hline
\end{tabular}




\section{Discussion}

The current study demonstrates that retrospective symptom measurement by end-ofday diaries as well as an end-of-week questionnaire (GSRS), leads to higher scores when compared to electronic momentary symptom assessment (i.e. by ESM), in patients with IBS. This difference is most pronounced for abdominal pain and flatulence. Abdominal pain scores in the end-of-day dairies were similar to maximum pain reporting in ESM, indicating that subjects tend to report peak pain rather than average pain scores of the day in an end-of-day diary. This was not found for the other tested GI symptoms. With regard to GSRS versus ESM, in addition to abdominal pain and flatulence, also nausea, heartburn and bloating scores were higher in the retrospective method compared to momentary assessment. Furthermore, affective complaints in general did not lead to a larger difference in abdominal pain reporting between the end-of-day diary and ESM. However, within each method, IBS patients with panic disorder reported higher abdominal pain and bloating scores compared to those without.

The reporting of peak rather than average abdominal pain has been described previously by Weinland et al. ${ }^{16}$ for IBS patients, and by Redelmeier et al. ${ }^{26}$ studying abdominal pain of other origin, i.e. colonoscopy and lithotripsy associated pain. The observed patient preferences of peak pain reporting provide more understanding with regard to pain memory and perceived relevance in IBS patients. Interestingly, peak reporting was not found for nausea, bloating, belching and flatulence. The current findings may imply that pain is more prone to recall bias than other GI symptoms in IBS and may also be related to a considerable affective component in pain perception. However, it should be noted that some symptoms, especially nausea and belching, were less prevalent, and consequently the present study may lack power to identify potential differences between the methods for these symptoms. Accordingly it might be interesting to assess a symptom as nausea in a population of functional dyspepsia patients in order to investigate whether a larger difference between ESM and end-ofday diaries can be observed.

When comparing ESM scores to retrospective end-of-week GSRS scores, even larger differences were found in comparison to ESM versus end-of-day diary scores, indicating that questionnaires with a longer recall period are associated with higher symptom scores in IBS patients. This is in line with previous research describing differences between end-of-day and end-of-week symptoms assessment in IBS. ${ }^{4}$ When analysing ESM data in more detail, GI symptoms in IBS patients were found to fluctuate considerably during the day and during the week. The 7-day mean of ESM data is an average of days with higher and lower symptom burden. Subjects are prone to base their scores on symptomatic days when filling in the GSRS, equivalent to the peak-pain tendency in the end-of-day diaries. This tendency may underlie the recall bias in 
retrospective methods. The differences between ESM and GSRS were tested parametrically on subject-level. However, non-parametric testing did not lead to different outcomes. The standard deviations of GSRS and ESM 7-day mean scores, indicating variation between subjects, in this analysis were broad, but differences between methods were highly significant.

Prevalent psychological factors in IBS, in particular anxiety, have been reported to affect pain processing, memory and interpretation. ${ }^{11}$ To investigate how anxiety may influence the difference between the retrospective (based on memory) and momentary assessment of abdominal pain, we included IBS patients with and without a diagnosis of comorbid panic disorder in the current study. Although, the overall scores for abdominal pain were higher in patients with compared to the group without panic disorder, only the presence of panic disorder in male subjects was found to be a significant predictor of a higher pain score difference between ESM and end-of-day diary. However, this predictor was no longer significant when corrected for multiple testing and should therefore be interpreted carefully. Furthermore, given the higher abdominal pain scores in IBS patients with panic disorder compared to those without, anxiety should be seen as a stable trait factor, which may less depend from momentarily fluctuating contextual influences in IBS. It has to be acknowledged that in the current study the IBS patients with panic disorder were overrepresented relative to the general IBS population. Therefore our data need to be replicated by future studies in large non-selected IBS cohorts.

In addition to higher overall scores and peak-symptom reporting in paper end-of-day diaries, this method is also compromised by high fake compliance, as demonstrates previously by Stone et al. ${ }^{12}$ Therefore, serious concern has risen with respect to the validity of retrospective paper end-of-day diaries. Fake compliance is excluded in ESM, since subjects can only respond within 10 minutes after the beep, and moreover, actual compliance can be monitored. We considered a subject to be compliant on a specific day if at least 6 out of 10 beep-questionnaires had been completed and found a good compliance rate for ESM, i.e. $76.8 \%$ for the total study population.

Certain limitations of the present study should be mentioned. In the current study we have not assessed differences in reporting of consistency and frequency of defecation between the symptom assessment methods, while disordered defecation is a key symptom of IBS. Furthermore, the use of ESM may lead to higher patient burden compared to end-of-day paper diaries. This may lead to selection bias, because patient may decide not to participate beforehand. The number of questions asked was high and should be reduced in future investigations. The time needed to complete a beepquestionnaire should be less than 2 minutes. Questions used in ESM were selected based on available questionnaires, such as the end-of-day diary ${ }^{2,3,22}$ and the GSRS, ${ }^{25,27}$ 
and were previously not psychometrically tested for momentary symptom assessment. Therefore, future studies evaluating the potential of ESM for symptom assessment in IBS should study psychometric properties, regarding validity and reliability of the instrument, which were not subject to analysis in this preliminary study. It has to be acknowledged that for the comparison of ESM and end-of-day diaries, it was necessary to rescale the ESM data from a 7 to a 5-point scale, which could cause a distortion of the results. However, the Cronbach's $\alpha$ for ESM GI symptom scores did not change after rescaling, and moreover, comparable results were found by comparing ESM (nonrescaled data) with GSRS scores. Finally, the exploratory aim of the current study has resulted in a small sample size. However, this has not hampered the multilevel analyses, which are based on repeated measures.

Our results demonstrate certain advantages of ESM as a new digital symptom assessment tool in IBS patients, regardless of the presence of psychological complaints. Nevertheless, further research is needed to optimize the content and number of questions for the assessment of IBS symptoms and to validate the tool as well as the selected questions within the target population, before ESM can be implemented in medical science and clinical practice.

Because of the repeated measures, ESM offers the advantage to study symptom fluctuation during the day and to investigate possible triggers of pain or other symptoms in future investigations, including for example ecological and psychosocial factors as well as linkage to bowel habits. This insight may lead to improvement of therapies. Previously has been found that IBS symptom episodes on average last several hours, ${ }^{16}$ which indicates that repeated measures over the day by ESM are suitable to capture the symptom variability in these patients. All patients in the current study had a debriefing interview with the researcher, and acknowledged that the number of repeated measures was sufficient to adequately measure the GI symptom variation over the day. Furthermore, ESM could be used for more detailed evaluation of treatment response or for intervention purposes in behavioural therapies, in the context of personalized medicine, by giving IBS patients specific feedback with regard to triggers of symptoms identified by ESM. This has recently been successfully applied in a group of individuals with depression. ${ }^{28}$

\section{Conclusion}

IBS patients report higher abdominal pain and flatulence scores in end-of-day diaries when compared to day-average scores of momentary symptom assessment by ESM, with a preference of peak-pain reporting at the end of the day. In retrospective symptom questionnaires that cover a one-week period, the difference of symptom 
reporting when compared to electronic ESM is even higher for abdominal pain and the other GI symptoms. Affective comorbidity in IBS leads to higher abdominal pain and bloating scores compared to IBS patients without, but did not strongly influence differences in abdominal pain reporting between tested symptom assessment methods. 


\section{References}

1. Spiegel B, Bolus R, Harris LA, Lucak S, Naliboff B, Esrailian E, Chey WD, Lembo A, Karsan H, Tillisch K, Talley J, Mayer E, Chang L. Measuring irritable bowel syndrome patient-reported outcomes with an abdominal pain numeric rating scale. Aliment Pharmacol Ther. 2009;30:1159-70.

2. Thijssen AY, Jonkers DM, Leue $C$, van der Veek PP, Vidakovic-Vukic $M$, van Rood YR, Clemens $C H$, Masclee AA. Dysfunctional cognitions, anxiety and depression in irritable bowel syndrome. J Clin Gastroenterol. 2010;44:e236-41.

3. Ludidi S, Mujagic Z, Jonkers D, Keszthelyi D, Hesselink M, Kruimel J, Conchillo J, Masclee A. Markers for visceral hypersensitivity in patients with irritable bowel syndrome. Neurogastroenterol Motil. 2014; 26:1104-11.

4. Lackner JM, Jaccard J, Keefer L, Firth R, Carosella AM, Sitrin M, Brenner D; Representing the IBSOS Research Group. The accuracy of patient-reported measures for Gl symptoms: a comparison of real time and retrospective reports. Neurogastroenterol Motil. 2014;26:1802-11.

5. U.S. Department of Health and Human Services FaDA, Center for Drug Evaluation and Research (CDER). Guidance for Industry Irritable Bowel Syndrome - Clinical Evaluation of Drugs for Treatment2012. http://wwwfdagov/downloads/Drugs/Guidances/UCM205269pdf.

6. Bradburn NM, Rips LJ, Shevell SK. Answering autobiographical questions: the impact of memory and inference on surveys. Science. 1987;236:157-61.

7. Bower GH. Mood and memory. Am Psychol. 1981;36:129-48.

8. Babel P, Pieniazek L, Zarotynski D. The effect of the type of pain on the accuracy of memory of pain and affect. Eur J Pain. 2015;19:358-68.

9. Bolger N, Davis A, Rafaeli E. Diary methods: capturing life as it is lived. Annu Rev Psychol. 2003;54: 579-616.

10. Grzesiak M, Beszłej JA, Mulak A, Szechiński M, Szewczuk-Bogusławska M, Waszczuk E, Kantorska M, Frydecka D. The lifetime prevalence of anxiety disorders among patients with irritable bowel syndrome. Adv Clin Exp Med. 2014;23:987-92.

11. Gorczyca R, Filip R, Walczak E. Psychological aspects of pain. Ann Agric Environ Med. 2013;Spec no. 1:23-7.

12. Stone AA, Shiffman S, Schwartz JE, Broderick JE, Hufford MR. Patient compliance with paper and electronic diaries. Control Clin Trials. 2003;24:182-99.

13. Csikszentmihalyi M, Larson R. Validity and reliability of the Experience-Sampling Method. J Nerv Ment Dis. 1987;175:526-36.

14. Stone A, Shiffman S. Ecological Momentary Assessment (EMA) in behavioral medicine. Ann Behav Med. 1994;16(199-202).

15. Myin-Germeys I, Oorschot M, Collip D, Lataster J, Delespaul P, van Os J. Experience sampling research in psychopathology: opening the black box of daily life. Psychol Med. 2009;39:1533-47.

16. Weinland SR, Morris CB, Hu Y, Leserman J, Bangdiwala SI, Drossman DA. Characterization of episodes of irritable bowel syndrome using ecological momentary assessment. Am J Gastroenterol. 2011;106: 1813-20.

17. Longstreth GF, Thompson WG, Chey WD, Houghton LA, Mearin F, Spiller RC. Functional bowel disorders. Gastroenterology. 2006;130:1480-91.

18. 4th. ed., text rev.; DSM-IV-TR; American Psychiatric Association. 2000

19. Zigmond AS, Snaith RP. The hospital anxiety and depression scale. Acta Psychiatr Scand. 1983;67: 361-70.

20. Bjelland I, Dahl AA, Haug TT, Neckelmann D. The validity of the Hospital Anxiety and Depression Scale. An updated literature review. J Psychosom Res. 2002;52:69-77.

21. Toner BB, Stuckless N, Ali A, Downie F, Emmott S, Akman D. The development of a cognitive scale for functional bowel disorders. Psychosom Med. 1998;60:492-7.

22. Mujagic Z, Ludidi S, Keszthelyi D, Hesselink MA, Kruimel JW, Lenaerts K, Hanssen NM, Conchillo JM, Jonkers DM, Masclee AA. Small intestinal permeability is increased in diarrhoea predominant IBS, while alterations in gastroduodenal permeability in all IBS subtypes are largely attributable to confounders. Aliment Pharmacol Ther. 2014;40:288-97. 
23. Myin-Germeys I, Birchwood M, Kwapil T. From environment to therapy in psychosis: a real-world momentary assessment approach. Schizophr Bull. 2011;37:244-7.

24. Courvoisier DS, Eid M, Lischetzke T. Compliance to a cell phone-based ecological momentary assessment study: the effect of time and personality characteristics. Psychol Assess. 2012;24:713-20.

25. Revicki DA, Wood M, Wiklund I, Crawley J. Reliability and validity of the Gastrointestinal Symptom Rating Scale in patients with gastroesophageal reflux disease. Qual Life Res. 1998;7:75-83.

26. Redelmeier DA, Kahneman D. Patients' memories of painful medical treatments: real-time and retrospective evaluations of two minimally invasive procedures. Pain. 1996;66:3-8.

27. Svedlund J, Sjodin I, Dotevall G. GSRS--a clinical rating scale for gastrointestinal symptoms in patients with irritable bowel syndrome and peptic ulcer disease. Dig Dis Sci. 1988;33:129-34.

28. Kramer I, Simons CJ, Hartmann JA, Menne-Lothmann C, Viechtbauer W, Peeters F, Schruers K, van Bemmel AL, Myin-Germeys I, Delespaul P, van Os J, Wichers M. A therapeutic application of the experience sampling method in the treatment of depression: a randomized controlled trial. World Psychiatry. 2014;13:68-77. 


\section{Supplementary data}

Table S9.1 End-of-day diary scores on 7 days with and 7 days without ESM, differences tested using paired samples t-test.

\begin{tabular}{lccc}
\hline $\begin{array}{l}\text { End-of-day Gl symptom diary } \\
\text { Gl symptoms (mean } \pm \text { SD) }\end{array}$ & Days with ESM & Days without ESM & p-value \\
\hline Abdominal pain & $2.53 \pm 1.01$ & $2.43 \pm 1.03$ & NS \\
Discomfort & $2.54 \pm 1.01$ & $2.47 \pm 0.97$ & NS \\
Nausea & $1.31 \pm 0.76$ & $1.41 \pm 0.82$ & NS \\
Bloating & $2.35 \pm 1.20$ & $2.42 \pm 1.18$ & NS \\
Belching & $1.41 \pm 0.89$ & $1.44 \pm 0.88$ & NS \\
Flatulence & $2.16 \pm 0.99$ & $2.40 \pm 1.16$ & NS \\
Constipation & $1.58 \pm 1.02$ & $1.48 \pm 0.83$ & NS \\
Diarrhoea & $1.27 \pm 0.70$ & $1.36 \pm 0.82$ & NS \\
\hline
\end{tabular}
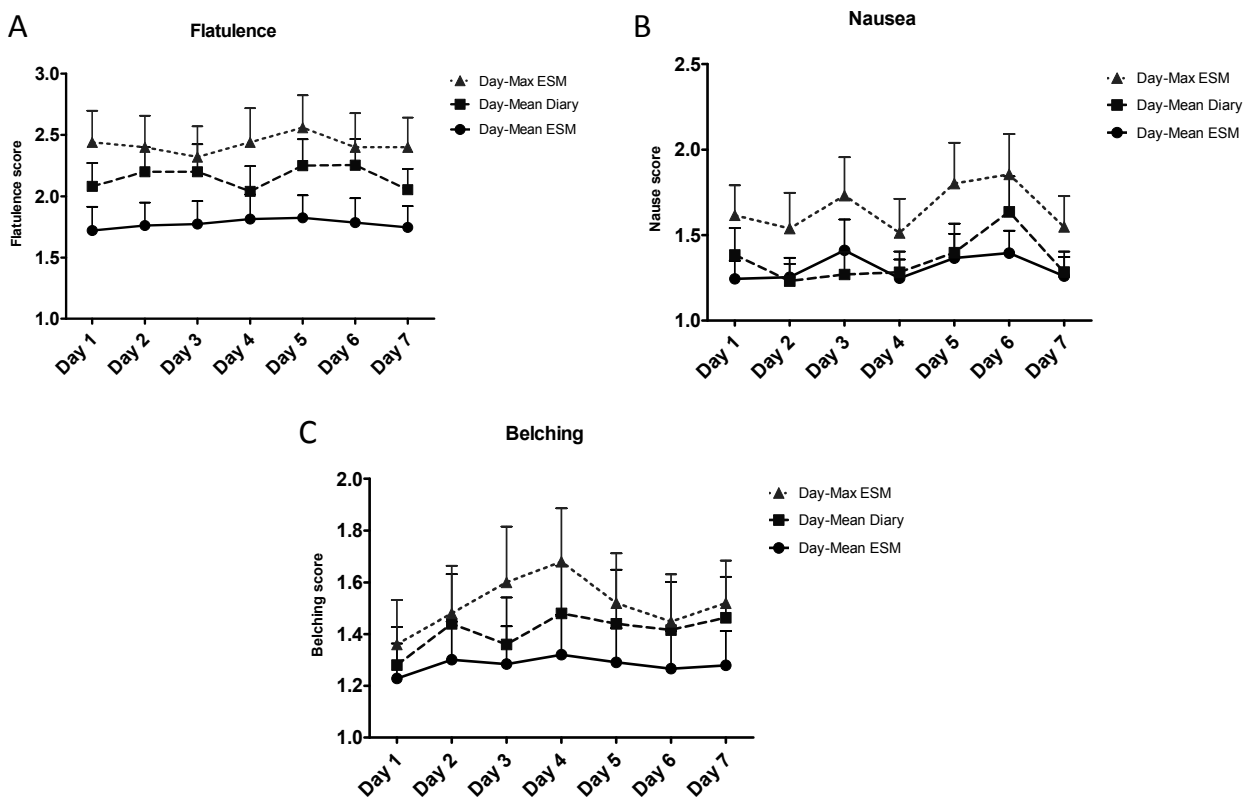

Figure S9.1 End-of-day diary scores compared to ESM day-mean and day-maximum scores, for flatulence (A) nausea (B) and belching (C). Differences tested for both ESM day-mean and ESM day-max scores vs. end-of-diary scores, using linear mixed models 


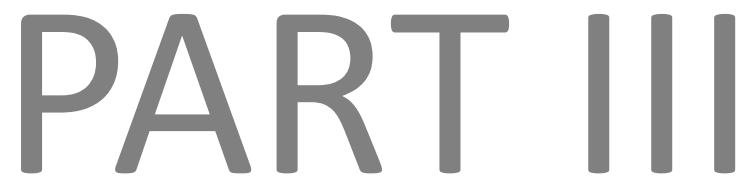

Biomarkers 


\section{Chapter 10}

\section{A novel faecal and plasma biomarker panel for IBS}

A novel gut health biomarker panel for irritable bowel syndrome and the application in the general population

Zlatan Mujagic*, Ettje F Tigchelaar*, Alexanda Zhernakova, Thomas Ludwig, Javier Ramiro-Garcia, Agnieszka Baranska, Morris A Swertz, Ad AM Masclee, Cisca Wijmenga, Frederik J van Schooten, Agnieszka Smolinska^, Daisy MAE Jonkers^^

$* /{ }^{\wedge}$ authors share co-first and co-last authorship, respectively

\section{Submitted}




\section{Abstract}

\section{Objective}

Biological markers for the diagnosis of functional gastrointestinal (GI) disorders, such as irritable bowel syndrome (IBS) that correlate well with GI symptom severity, are lacking. The objective of the present study was to identify and validate a non-invasive biomarker panel, related to several domains of gut health associated with the pathophysiology of IBS, that discriminates IBS patients from healthy controls (HC), and correlates with $\mathrm{GI}$ symptom severity.

\section{Methods and findings}

Six plasma cytokines (IL-1 $\beta$, IL-6, IL-8, IL-10, IL-12p70, TNF- $\alpha$ ), plasma citrulline, and eight faecal markers (human $\beta$-defensin 2 (HBD2), calprotectin, chromogranin A (CgA), short chain fatty acids: acetate, propionate, butyrate, valerate, and caproate, were measured in a cohort of 196 clinical IBS patients and $160 \mathrm{HC}$ without GI symptoms. A combination of biomarkers, which best discriminates between IBS and HC was identified and validated in an independent validation set and by permutation testing. $A$ non-invasive 8-item biomarker panel consisting of IL-1 $\beta$, IL-6, IL12p70, TNF- $\alpha, \operatorname{CgA}$, HBD2, calprotectin and caproate, was identified to discriminate IBS form HC with high sensitivity (88.1\%) and specificity (86.5\%), with an area under the ROC curve of 0.89 . The results for the IBS subtypes were comparable. Moreover, a moderate correlation was found for biomarker panel and GI symptom scores in the IBS cohort $(r=0.59$, $\mathrm{p}<0.001$ ). Furthermore, to test a possible application of the biomarker panel, all biomarkers were measured in a general population cohort of 958 subjects. In subjects of this cohort with low to moderate GI symptom burden a moderate correlation ( $r=0.51, p=0.003$ ) was found between the 8-item biomarker panel and several $\mathrm{GI}$ symptom scores.

\section{Conclusion}

A novel non-invasive multi-domain biomarker panel, with high positive predictive value for IBS, has been identified and validated, which correlated moderately to GI symptom severity in IBS patients and general population subjects. 


\section{Introduction}

Interest in 'gut health' is rapidly emerging, not only from a scientific but also from a societal and individual perspective. ${ }^{1}$ Gastrointestinal (GI) symptoms and disorders occur frequently in the general population and may reflect compromised gut health. ${ }^{2}$ Although gut health is difficult to define, five major domains related to a healthy $\mathrm{GI}$ system have been proposed by Bischoff. These domains include effective digestion and absorption, normal and stable microbiota, effective immune status, absence of $\mathrm{GI}$ illness and status of general well-being. ${ }^{1}$ As various underlying mechanisms may affect these domains, it is challenging to identify and validate markers for gut health. This is particularly relevant when healthy subjects are compared with subjects with functional GI disorders, or when evaluating potential benefits of dietary interventions. For organic GI diseases, diagnostic tools and markers are generally available in daily clinical practice, such as GI endoscopy, histological examination of tissue samples or biomarkers (e.g. anti-transglutaminase antibodies). ${ }^{3,4}$ However, objective biological markers that aid the diagnostic process and facilitate follow up and assessment of treatment efficacy of functional Gl disorders, such as Irritable Bowel Syndrome (IBS), are lacking. Neither are markers available that correlate well with GI symptom severity.

The Rome III symptom criteria are the current gold standard for the diagnosis of IBS. But, although the Rome committee emphasizes that IBS is not a diagnosis of exclusion and should be based on symptom criteria, in daily clinical practice endoscopy, CT or ultrasound imaging, blood, faecal or other analyses are frequently used to exclude organic disorders. ${ }^{7}$ Therefore, there is increasing interest in reliable biomarkers that can aid the diagnosis of IBS. A clinically and scientifically relevant biomarker for IBS should be able to a) discriminate IBS patients from healthy subjects, b) discriminate IBS patients from subjects with organic Gl disorders, and c) correlate with severity of GI symptoms, to be useful for monitoring of treatment efficacy. Finding objective biomarkers that correlate with the severity of GI symptoms in IBS is also an important step towards suitable biomarkers for functional GI complaints and gut health in the general population.

Up to now, use of non-invasive single biomarkers for IBS has led to only moderate results. ${ }^{5,6}$ Therefore, recent studies focused on biomarker panels. ${ }^{8}$ To date, a specificity and sensitivity of $88 \%$ and $50 \%$, respectively, has been reported for a 10 item-serum marker panel, ${ }^{9}$ and of $64 \%$ and $81 \%$, respectively, for a 34 item-serum/genetic marker panel for the discrimination of IBS patients versus healthy controls. ${ }^{10}$ Though promising, biomarkers with higher specificity and sensitivity are needed for daily clinical practice and scientific research. We postulated that combining markers that reflect the multifactorial pathophysiology of IBS may lead to better results. Strong evidence exists for a combination of rather subtle pathophysiological changes, such as low-grade 
mucosal and systemic immune activation, intestinal barrier dysfunction, dysregulation in neural and neuro-hormonal signalling and altered microbiota and host-microbe interaction, that contribute to symptom generation in IBS. ${ }^{11}$ These mechanisms do overlap with the domains of gut health as described by Bischoff. ${ }^{1}$

In the current study, we hypothesized that biomarkers related to several domains of gut health and associated with the pathophysiology of IBS, can be combined and used as a highly sensitive and specific biomarker panel to discriminate clinically diagnosed IBS patients form healthy controls. Secondly, we hypothesized that this biomarker panel correlates with GI symptom severity, not only in IBS patients derived from a clinical setting with relatively high symptom burden, but also in subjects with mild to moderate $\mathrm{Gl}$ symptoms from the general population.

The primary objective of the present study was to assess levels of selected plasma and faecal biomarkers, related to several domains of gut health, and to identify and validate a non-invasive biomarker panel, which best discriminates IBS patients from healthy controls. The secondary objective was to correlate the biomarker panel to Gl symptom severity in clinical IBS patients as well as in a large general population cohort.

\section{Methods}

Two large Dutch cohorts were used for the current study, i.e. a clinical cohort of IBS patients and healthy controls without GI symptoms (Maastricht IBS (MIBS) cohort), ${ }^{12,13}$ and a general population cohort (LifeLines (LL) DEEP). ${ }^{14}$ From both cohorts, subjects were included in the analyses if faecal and plasma samples and completed GI symptom scores were available. Identification and validation of the biomarker panel was conducted in clinical IBS patients and healthy controls of the MIBS cohort. The correlation of the biomarker panel to GI symptoms was evaluated in both cohorts. All study participants gave written informed consent prior to inclusion. Study protocols were approved by the Maastricht University Medical Center+ (MUMC+) and University Medical Centre Groningen (UMCG) Ethics Committees, respectively, and were in compliance with the revised Declaration of Helsinki (64th WMA General Assembly, Fortaleza, Brazil, 2013). The MIBS cohort was registered in the US National Library of Medicine (http://www.clinicaltrials.gov, NCT00775060).

\section{Study participants; MIBS cohort}

IBS patients, between 18 and 75 years of age, diagnosed by their treating physician and fulfilling Rome III criteria, were recruited via the outpatient gastroenterologyhepatology clinic of MUMC+, a secondary and tertiary referral centre, and via general practitioners in the area of Maastricht. Medical history was taken and GI endoscopy 
with biopsies, abdominal imaging and/or blood, breath and faecal analyses were performed to exclude organic disease, when indicated. Patients with a history of abdominal surgery, apart from appendectomy, laparoscopic cholecystectomy and hysterectomy, were excluded. Patients were assigned to IBS subtypes based on predominant bowel habits according to the Rome III criteria: diarrhoea (IBS-D), constipation (IBS-C), mixed stool pattern (IBS-M) and unspecified subtype (IBS-U). Age and sex matched healthy controls $(\mathrm{HC})$ were enrolled via public advertising. A brief medical history was taken to exclude the presence of previous or current GI disorders or complaints. All study participants completed questionnaires regarding demographic characteristics and lifestyle factors. A 14-day end-of-day GI symptom diary was completed, addressing symptoms of abdominal discomfort and pain, nausea, bloating, belching, flatulence, diarrhoea and constipation, on a 1-to-5 point Likert scale. ${ }^{13}$

\section{Study participants; LL DEEP cohort}

Study participants above 18 years of age and regardless of their medical background or current complaints were enrolled from a large general population cohort in the Netherlands, i.e. LifeLines. ${ }^{15}$ In a subgroup of the cohort, referred to as LifeLines Detailed Extensive Examination of Participants (LL DEEP), among others subjects collected faecal samples and completed self-report questionnaires on demographics and lifestyle factors, as well as a 7-day GI symptom diary, which was identical to the 14-day diary used in the MIBS cohort. ${ }^{14}$ Information on clinical diagnoses of IBS was not available for this general population cohort.

\section{Selection of biomarkers}

Forty-three potential biomarkers were selected based on extensive literature search, using the following selection criteria: i) markers are related to one or more gut health domains as described by Bischoff ${ }^{1}$; ii) they potentially discriminate between subjects with healthy gut function and IBS patients; and iii) can be measured in blood or faecal samples collected according to generally accepted and applicable procedures for bio sample collection. Based on expert panels, focusing on relevance and feasibility of the markers, fifteen biomarkers were selected to be measured (in both cohorts). Plasma citrulline, a marker of functional enterocyte mass of the small bowel, was selected as indicator of the absorptive ${ }^{16,17}$ and barrier function of the small intestine. ${ }^{18,19}$ Nonstimulated plasma cytokine levels, i.e. IL-1 $\beta, \mathrm{IL}-6, \mathrm{IL}-8, \mathrm{IL}-10, \mathrm{IL}-12 \mathrm{p} 70$ and TNF- $\alpha$, were selected as markers of systemic immune activation. ${ }^{20,21}$ Faecal calprotectin, a calcium and zinc-binding protein, was selected as an indicator of intestinal inflammation, proportional to neutrophil migration towards the intestinal tract. ${ }^{22-24}$ Faecal human B-defensin 2 (HBD2), a human antimicrobial peptide produced by among others intestinal epithelial cells in response to (pathogenic) bacteria, was selected as an indicator of host defence against microbes. ${ }^{25,26}$ Faecal short chain fatty acids (SCFAs), 
i.e. acetate, propionate, butyrate, valerate, and caproate, are products of microbial fermentation of non-digested oligosaccharides in the colon, and were selected as indicators of gut intraluminal metabolic activity. SCFAs have been associated to multiple pathological and physiological mechanisms in humans, e.g. modulation of inflammation, satiety, carcinogenesis, and are an important energy source for colonocytes. $^{27,28}$ Faecal chromogranin A ( $\left.\mathrm{CgA}\right)$, was selected as an indicator of the intestinal neuroendocrine cell activity. This peptide is produced by among others enterochromaffin cells, and is co-localized in storage granules with serotonin. ${ }^{24,29-31}$

\section{Measurement of biomarkers}

Faecal and blood samples were collected using standardized collection procedures. Venous blood was collected in $\mathrm{K}_{2}$ EDTA (BD Vacutainer ${ }^{\circledR}$ ) tubes and centrifuged to obtained plasma supernatants, which were aliquoted and frozen at $-80^{\circ} \mathrm{C}$ until analysis. In the same time period, subjects collected stool samples, which were aliquoted and stored at $-80^{\circ} \mathrm{C}$ within 24 hours after defecation. All markers were measured simultaneously to avoid additional thaw cycles, by 'Medische Laboratoria Dr. Stein \& Colleges', The Netherlands. Calprotectin and HBD2 were assessed using a commercial enzyme-linked immunosorbent assay (ELISA, by Bühlmann Laboratories, Switzerland, and Immunodiagnostik AG, Germany, respectively), ${ }^{24,26}$ and $\operatorname{CgA}$ using a commercial radioimmunoassay (RIA, Euro-Diagnostica, Sweden), ${ }^{24}$ as described previously. ${ }^{24,26}$ Faecal SCFAs, i.e. acetate, propionate, butyrate, valerate and caproate, were measured by gas chromatography-mass spectrometry (GC-MS) according to the method described by Garciá-Villalba et al. ${ }^{32}$ Concentrations of plasma citrulline were determined by high pressure liquid chromatography (HPLC) fluorescence detection, as described previously. ${ }^{16}$ Plasma cytokines, i.e. IL-1 $\beta$, IL-6, IL-8, IL-10, IL-12p70 and TNF- $\alpha$, were measured by ProcartaPlex ${ }^{\mathrm{TM}}$ multiplex immunoassay (eBioscience, USA), as described previously. 33,34

\section{Data and statistical analysis}

Levels of individual biomarkers were compared between groups using the MannWhitney $U$ test, due to left skewed distributions, with post-hoc correction for multiple testing by the Benjamini-Hochberg step-up procedure. ${ }^{35}$ The method of multiple imputations was implemented as part of the statistical procedure for biomarker levels that were below the detection limit. ${ }^{36}$ These were randomly (with uniform distribution) replaced by levels ranging from zero till limit of detection. The assignment of values below detection limit was repeated 2000 times. All statistical results were calculated as average values over the 2000 iterations.

The data set of the MIBS cohort was then used for identification and validation of a biomarker panel that best discriminates between IBS and HC, using a supervised technique, namely Partial Least Square Discriminant Analysis (PLS-DA). ${ }^{37}$ This strategy 
for supervised data analysis via PLS-DA involved a random division of the MIBS cohort into a representative training set ( $80 \%$ of samples per group) and a validation set ( $20 \%$ of samples per group), as described by Guyon. ${ }^{38}$ Within the training set we applied double cross-validation procedure for model optimization, the most discriminatory biomarker selection and for development of the final PLS-DA classification model. ${ }^{39,40}$ The latter was done in the independent validation set of the MIBS cohort (the randomly separated $20 \%$ of samples not used in any optimization steps), by testing the performance of selected biomarker panel to classify individuals as being an IBS patient or HC.

Note, that commonly used cross-validation leads to optimistic bias (i.e. too low error estimate). ${ }^{41}$ Thus, it was not used in our analyses for evaluating the predictive power of the PLS-DA model.

Furthermore, linear correlations between the most discriminatory biomarker panel and a set of Gl symptoms, including all items of the Gl symptom diary were tested in the MIBS and in the general population LL-DEEP cohort, using canonical correlation analysis $(C C A),{ }^{42}$ which is an extension of bi-variate correlation. CCA calculates a linear correlation that best explains the variability both within and between the biomarker panel and the measured GI symptoms.

\section{Results}

\section{Identification and validation of the most discriminative biomarker panel for IBS versus HC}

Baseline characteristics for 196 clinical IBS patients and 160 age and gender matched HC without GI symptoms (MIBS cohort) are presented in Table 10.1. All GI symptom scores were higher in IBS patients compared to HC. The scores for constipation and diarrhoea were significantly altered, in line with the IBS subtypes (data not shown).

The biomarker data for IBS and HC subjects of the total MIBS cohort are presented in Table 10.2. Based on univariate comparisons, non-stimulated IL- $1 \beta$ and IL- 6 were significantly lower, while IL-12p70 was significantly higher in plasma samples of IBS patients relative to $\mathrm{HC}$. In faecal samples, $\mathrm{CgA}$ and calprotectin were significantly increased, while HBD2, and the SCFAs, valerate and caproate, were significantly decreased in IBS patients compared to HC. These differences remained statistically significant after correction for multiple testing. 
Table 10.1 Baseline characteristics of the MIBS cohort groups (i.e. IBS patients vs. HC).

\begin{tabular}{|c|c|c|}
\hline Parameter & IBS $(n=196)$ & $\mathrm{HC}(\mathrm{n}=160)$ \\
\hline \multicolumn{3}{|l|}{ Demographics and Lifestyle } \\
\hline Age (mean years $\pm S D)$ & $44.8 \pm 16.4$ & $43.9 \pm 19.2$ \\
\hline Female sex $(\%)$ & 70.4 & 61.3 \\
\hline $\mathrm{BMI}\left(\right.$ mean $\left.\mathrm{kg} / \mathrm{m}^{2} \pm \mathrm{SD}\right)$ & $25.0 \pm 4.6^{\mathrm{a}}$ & $24.0 \pm 3.9$ \\
\hline Duration of IBS symptoms (mean years \pm SD) & $13.6 \pm 10.8$ & - \\
\hline IBS subtype: IBS-D / IBS-C / IBS-M / IBS-U (n) & $71 / 34 / 78 / 13$ & - \\
\hline Current or previous smoker (\%) & $51.6^{\mathrm{c}}$ & 39.3 \\
\hline Alcohol abstainers: 0 units/week (\%) & $42.1^{c}$ & 19.1 \\
\hline Moderate alcohol use: 1-15 units/week (\%) & $54.7^{b}$ & 70.7 \\
\hline \multicolumn{3}{|c|}{ GI symptoms assessed by end-of-day diary, 14-day mean \pm SD } \\
\hline Abdominal pain & $2.3 \pm 0.9^{c}$ & $1.1 \pm 0.2$ \\
\hline Abdominal discomfort & $2.4 \pm 0.8^{c}$ & $1.1 \pm 0.2$ \\
\hline Bloating & $2.2 \pm 0.9^{c}$ & $1.1 \pm 0.2$ \\
\hline Belching & $1.6 \pm 0.7^{c}$ & $1.1 \pm 0.3$ \\
\hline Nausea & $1.6 \pm 0.8^{c}$ & $1.0 \pm 0.1$ \\
\hline Flatulence & $2.3 \pm 0.9^{c}$ & $1.3 \pm 0.5$ \\
\hline Constipation & $1.5 \pm 0.7^{c}$ & $1.1 \pm 0.2$ \\
\hline Diarrhoea & $1.5 \pm 0.6^{c}$ & $1.0 \pm 0.1$ \\
\hline
\end{tabular}

Differences tested with independent samples t-test and Pearson $\mathrm{Chi}^{2} ;{ }^{a} \mathrm{p}<0.05 ;{ }^{b} \mathrm{p}<0.01 ;{ }^{\mathrm{c}} \mathrm{p}<0.001$ vs. HC

Table 10.2 Levels of biomarkers of the MIBS cohort (i.e. IBS patients versus HC).

\begin{tabular}{|c|c|c|c|}
\hline & $\begin{array}{c}\text { IBS } \\
(n=196)\end{array}$ & $\begin{array}{c}H C \\
(n=160)\end{array}$ & $p$-value \\
\hline \multicolumn{4}{|l|}{ Plasma biomarkers (median [Q1-Q3) } \\
\hline Citrulline $(\mu \mathrm{mol} / \mathrm{l})$ & $41.9(33.9 ; 50.5)$ & $39.3(33.0 ; 47.2)$ & NS \\
\hline $\mathrm{IL}-1 \beta(\mu \mathrm{g} / \mathrm{I})$ & $0.15(0.08 ; 0.71)$ & $0.19(0.10 ; 1.46)$ & 0.01 \\
\hline IL-6 ( $\mu \mathrm{g} / \mathrm{I})$ & $0.24(0.12 ; 0.36)$ & $0.52(0.20 ; 3.13)$ & $<0.001$ \\
\hline $\mathrm{IL}-8(\mu \mathrm{g} / \mathrm{I})$ & $1.69(0.95 ; 2.52)$ & $1.60(1.14 ; 2.45)$ & NS \\
\hline IL-10 ( $\mu \mathrm{g} / \mathrm{I})$ & $0.86(0.69 ; 1.09)$ & $0.84(0.61 ; 1.04)$ & NS \\
\hline $\mathrm{IL}-12 \mathrm{p} 70(\mu \mathrm{g} / \mathrm{I})$ & $2.19(1.21 ; 2.99)$ & $1.20(0.11 ; 2.00)$ & $<0.001$ \\
\hline TNF- $\alpha(\mu \mathrm{g} / \mathrm{I})$ & $0.14(0.07 ; 3.20)$ & $0.13(0.06 ; 1.53)$ & NS \\
\hline \multicolumn{4}{|l|}{ Faecal biomarkers (median [Q1-Q3]) } \\
\hline Chromogranin-A (CgA) (nmol/g) & $15.2(7.6 ; 45.8)$ & $9.4(6.3 ; 27.3)$ & 0.001 \\
\hline Calprotectin ( $\mu \mathrm{g} / \mathrm{g})$ & $37.3(18.7 ; 73.5)$ & $21.6(6.8 ; 47.8)$ & $<0.001$ \\
\hline Human $\beta$-defensin 2 (HBD2) (ng/g) & $31.3(18.8 ; 51.9)$ & $38.4(27.1 ; 60.5)$ & $<0.01$ \\
\hline SCFA (C2): Acetate $(\mu \mathrm{mol} / \mathrm{g})$ & $33.4(22.5 ; 47.1)$ & $35.7(25.9 ; 47.4)$ & NS \\
\hline SCFA (C3): Propionate ( $\mu \mathrm{mol} / \mathrm{g})$ & $9.5(6.8 ; 14.0)$ & $9.5(6.6 ; 13.8)$ & NS \\
\hline SCFA (C4): Butyrate $(\mu \mathrm{mol} / \mathrm{g})$ & $7.8(4.6 ; 13.2)$ & $9.4(5.9 ; 13.5)$ & NS \\
\hline SCFA (C5): Valerate ( $\mu \mathrm{mol} / \mathrm{g})$ & $1.2(0.8 ; 1.8)$ & $1.5(1.0 ; 2.1)$ & 0.01 \\
\hline SCFA (C6): Caproate $(\mu \mathrm{mol} / \mathrm{g})$ & $0.1(0.0 ; 0.5)$ & $0.3(0.1 ; 0.9)$ & $<0.001$ \\
\hline
\end{tabular}

Difference tested with Mann Whitney $U$ test (presented $p$-value). Statistically significant differences did not diminish after post-hoc correction for multiple testing by Benjamini-Hochberg step up procedure.

For identification of the most discriminative biomarker panel, the MIBS training cohort was used. A final panel of 8 biomarkers, i.e. IL-1 $\beta$, IL-6, IL12p70, TNF- $\alpha$, CgA, HBD2, calprotectin and caproate, was identified with PLS-DA to have the best predictive value 
for the IBS versus HC group. This panel is presented in Figure 10.1, wherein the regression coefficient level indicates the relative importance of the specific marker within the biomarker panel. Markers with a positive regression coefficient are decreased in the IBS group, and vice versa. The regression coefficients and the biomarker concentrations were used to calculate a total score for the biomarker panel per subject. This was done by multiplying the concentration of each biomarker as actually measured, by the level of importance of that specific marker within the panel obtained from the regression coefficient. The total score represents the probability of being IBS or HC. The values of these scores were found to be significantly different between the IBS versus the $\mathrm{HC}$ group in the validation set, as shown in Figure 10.2 $(p<0.001)$.

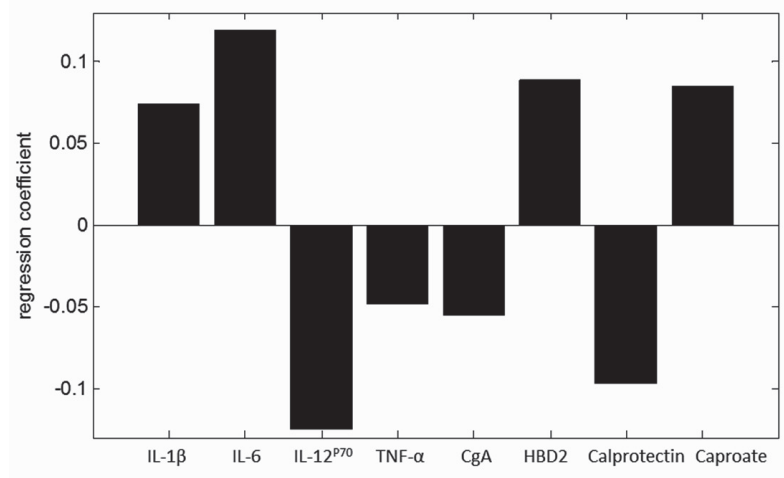

Figure 10.1 The final 8-item biomarker panel. The length of the bar, i.e. positive or negative regression coefficient level, indicates the relative importance of the specific marker within the biomarker panel. Positive markers are decreased in IBS patients compared to HC, and vice versa for the negative markers.

Thereafter, the pre-assigned independent validation set of the MIBS cohort of IBS patients and $\mathrm{HC}$ that were left out of the biomarker panel discovery step, was used to test the performance of the biomarker panel for the discrimination between IBS patients and $\mathrm{HC}$. This is illustrated by the receiver operating characteristic (ROC) curve in Figure 10.3, with an area under the curve (AUC) of 0.89. The line of the ROC curve represents the sensitivity and specificity of the classification model, which is derived from an algorithm representing all 8 biomarkers in the panel. The sensitivity and specificity at the optimal threshold for the diagnosis of IBS were $88.1 \%$ and $86.5 \%$, respectively, and the positive and negative predictive values were $94.4 \%$ and $74.0 \%$, respectively.

The results for the three largest IBS subtypes, for the set of 8 biomarkers, were comparable to the total IBS group, i.e. area under ROC curve of 0.86 for IBS-D, 0.80 for IBS-C and 0.89 for IBS-M in comparison to HC. These results were obtained by 
stratifying the number of individuals of each subtype in the validation set. Due to a small sample size in the IBS-U subtype $(n=13)$, the AUC was not calculated for this subtype.

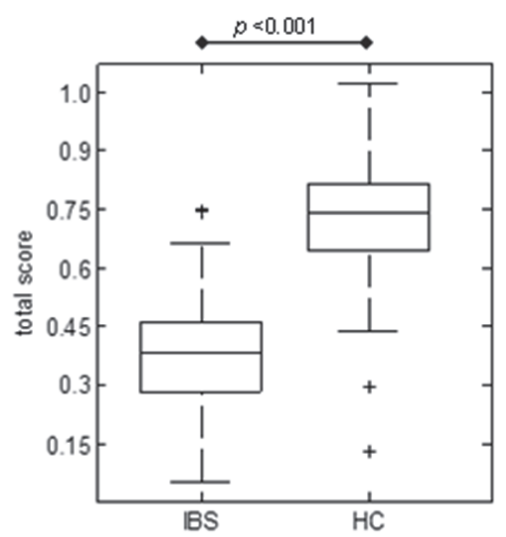

Figure 10.2 Box plot representing the total score obtained for the 8-item biomarker panel for the IBS and HC group. The total score was obtained by multiplying the real concentration of measured biomarkers in the validation set by the regression coefficient per marker (Figure 10.1). It represents the probability of being IBS or HC.

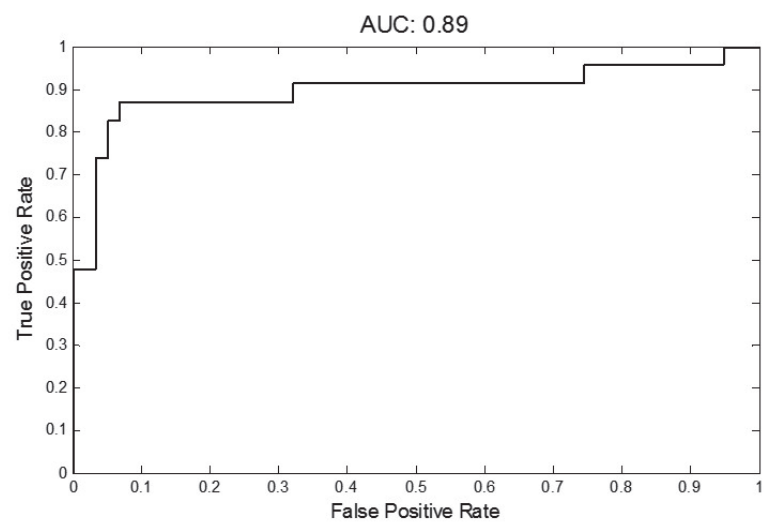

Figure 10.3 ROC curve, with an area under the curve (AUC) of 0.89 for the discrimination between IBS patients and $\mathrm{HC}$ in the validation set, using the final 8-item biomarker panel.

\section{Correlation between the biomarker panel and GI symptoms in the MIBS cohort}

In the total MIBS cohort (i.e. $160 \mathrm{HC}$ and 196 IBS patients), the strongest correlation, i.e. correlation coefficient of $0.59(p<0.001)$, between the 8 -item biomarker panel (Figure 
10.1) and a set of Gl symptoms, involving the following symptom scores: 14-day mean scores of abdominal pain, abdominal discomfort, bloating, flatulence and nausea. No correlations were found between the biomarker panel and the other GI symptoms assessed by the diary, i.e. belching, constipation and diarrhoea.

\section{Application of the biomarker panel in the general population LL DEEP cohort}

The potency of the biomarker set in the general population was evaluated by investigating the correlation between the 8-item biomarker panel (Figure 10.1) and GI symptoms assessed by the 7-day end-of-day GI symptom diary for the LL DEEP cohort (Table 10.3 and Table 10.4). Since Gl symptom severity scores in the total cohort ( $n=958$ ) were low, only subjects from the LL DEEP cohort with a GI symptom diary 7-day mean score for abdominal pain and discomfort above 1.5 were included in the correlation analysis. This cut-off value indicates the subject had at least 2 days with moderately-severe or 4 days with slightly-severe Gl symptoms during those 7 days. The cut-of value was further supported by data of the MIBS cohort in which $95 \%$ of healthy controls had end-of-day diary mean-scores below 1.5 for these Gl symptoms. A GI symptom diary mean score of 1.5 or higher was found in 221 (23\%) participants of the LL DEEP cohort. In this LL DEEP subgroup, the 8-item biomarker panel was found to correlate significantly with a set of GI symptoms, i.e. 7-day mean scores of abdominal pain, discomfort, constipation, belching and nausea, resulting in a correlation coefficient of $0.51(p=0.003)$.

Table 10.3 Baseline characteristics of the LL DEEP cohort: general population.

\begin{tabular}{|c|c|c|c|}
\hline Parameters of general population subjects & $\begin{array}{c}\text { Total group } \\
(n=958)\end{array}$ & $\begin{array}{c}\text { BM pos. group } \\
(n=226)\end{array}$ & $\begin{array}{c}\text { BM neg. group } \\
(n=143)\end{array}$ \\
\hline \multicolumn{4}{|l|}{ Demographics and Lifestyle } \\
\hline Age (mean years $\pm S D)$ & $45.2 \pm 13.2$ & $46.8 \pm 13.4$ & $44.3 \pm 12.8$ \\
\hline Female sex (\%) & 58.5 & 53.5 & 60.8 \\
\hline $\mathrm{BMI}\left(\right.$ mean $\left.\mathrm{kg} / \mathrm{m}^{2} \pm \mathrm{SD}\right)$ & $25.5 \pm 4.5$ & $25.8 \pm 5.1$ & $25.4 \pm 3.9$ \\
\hline Current smokers (\%) & 18.9 & 20.9 & 22.0 \\
\hline \multicolumn{4}{|c|}{ GI symptoms assessed by end-of-day diary, 7-day mean \pm SD } \\
\hline Abdominal pain & $1.2 \pm 0.4$ & $1.1 \pm 0.4$ & $1.2 \pm 0.5$ \\
\hline Abdominal discomfort & $1.3 \pm 0.5$ & $1.3 \pm 0.5$ & $1.3 \pm 0.5$ \\
\hline Bloating & $1.4 \pm 0.5$ & $1.3 \pm 0.6$ & $1.4 \pm 0.5$ \\
\hline Belching & $1.1 \pm 0.3$ & $1.1 \pm 0.3$ & $1.1 \pm 0.3$ \\
\hline Nausea & $1.1 \pm 0.3$ & $1.1 \pm 0.3$ & $1.1 \pm 0.2$ \\
\hline Flatulence & $1.6 \pm 0.6$ & $1.6 \pm 0.6$ & $1.8 \pm 0.6$ \\
\hline Constipation & $1.1 \pm 0.4$ & $1.2 \pm 0.4$ & $1.2 \pm 0.5$ \\
\hline Diarrhoea & $1.1 \pm 0.3$ & $1.1 \pm 0.3$ & $1.1 \pm 0.3$ \\
\hline
\end{tabular}

No statistically significant difference observed between the biomarker (BM) positive and negative group (groups defined based on a cut-off value of 0.75 ), tested with independent samples t-test and Pearson chi ${ }^{2}$. 
Table 10.4 Levels of biomarkers of the LL DEEP cohort: general population.

\begin{tabular}{|c|c|}
\hline & $\begin{array}{l}\text { Total group } \\
(n=958)\end{array}$ \\
\hline \multicolumn{2}{|l|}{ Plasma biomarkers (median [Q1-Q3]) } \\
\hline Citrulline $(\mu \mathrm{mol} / \mathrm{l})$ & $41.2(34.7 ; 49.1)$ \\
\hline $\mathrm{IL}-1 \beta(\mu \mathrm{g} / \mathrm{I})$ & $0.11(0.10 ; 1.00)$ \\
\hline $\mathrm{IL}-6(\mu \mathrm{g} / \mathrm{I})$ & $0.19(0.18 ; 0.20)$ \\
\hline $\mathrm{IL}-8(\mu \mathrm{g} / \mathrm{I})$ & $1.91(1.30 ; 2.77)$ \\
\hline IL-10 ( $\mu \mathrm{g} / \mathrm{I})$ & $0.89(0.56 ; 1.23)$ \\
\hline $\mathrm{IL}-12 \mathrm{p} 70(\mu \mathrm{g} / \mathrm{l})$ & $1.14(0.06 ; 2.25)$ \\
\hline TNF- $\alpha(\mu \mathrm{g} / \mathrm{I})$ & $0.08(0.07 ; 0.09)$ \\
\hline \multicolumn{2}{|l|}{ Faecal biomarkers (median [Q1-Q3]) } \\
\hline Chromogranin-A (CgA) (nmol/g) & $11.1(7.2 ; 23.4)$ \\
\hline Calprotectin $(\mu \mathrm{g} / \mathrm{g})$ & $31.6(10.5 ; 60.4)$ \\
\hline Human $\beta$-defensin 2 (HBD2) (ng/g) & $36.8(22.5 ; 62.2)$ \\
\hline SCFA (C2): Acetate $(\mu \mathrm{mol} / \mathrm{g})$ & $27.8(19.6 ; 47.1)$ \\
\hline SCFA (C3): Propionate $(\mu \mathrm{mol} / \mathrm{g})$ & $7.8(5.2 ; 11.6)$ \\
\hline SCFA (C4): Butyrate ( $\mu \mathrm{mol} / \mathrm{g})$ & $7.9(5.0 ; 12.5)$ \\
\hline SCFA (C5): Valerate $(\mu \mathrm{mol} / \mathrm{g})$ & $1.4(1.0 ; 2.0)$ \\
\hline SCFA (C6): Caproate ( $\mu \mathrm{mol} / \mathrm{g})$ & $0.3(0.1 ; 0.8)$ \\
\hline
\end{tabular}

\section{Discussion}

In the current study, a novel non-invasive biomarker panel has been identified and validated, with a high predictive value (area under ROC curve of 0.89 ) for the discrimination between clinically diagnosed IBS patients who fulfilled the Rome III criteria and healthy controls who did not have any GI complaints. The 8-item biomarker panel reflects multiple domains of gut health and correlated moderately $(r=0.59$, $\mathrm{p}<0.001)$ to $\mathrm{Gl}$ symptom severity in the IBS cohort, as well as in a general population cohort $(r=0.51, p=0.003)$.

In the search for a non-invasive biomarker panel for IBS, we measured 15 biomarkers in a large group of study participants. Eight markers (i.e. plasma IL-1 $\beta$, IL-6, IL-12p70, and faecal CgA, calprotectin, HBD2, valerate, and caproate) proved to be significantly altered in IBS patients compared to $\mathrm{HC}$, when tested by univariate analyses (Table 10.2). Of the 8 markers, 7 (valerate excluded) were part of the final 8-item biomarker panel (Figure 10.1) that best discriminated IBS patients from HC. Also TNF- $\alpha$ was identified as one of the discriminatory markers within the final biomarker panel, while the difference between IBS and HC in the univariate analysis was not statistically significant $(p>0.05)$. This supports the advantage of a biomarker panel that includes a combination of markers, over the use of single biomarkers for the positive identification of IBS patients in a cohort that also included healthy controls. 
Calprotectin and IL-12p70 were increased in IBS patients compared to $\mathrm{HC}$, pointing to a low-grade pro-inflammatory state in IBS, as has been reported previously for subgroups. ${ }^{20-23}$ Interestingly, IL-1 $\beta$ and IL- 6 were decreased in IBS patients compared to HC. IBS is a chronic GI disorder, and in contrast to IL-12p70, which is as proinflammatory cytokine associated with chronic inflammatory conditions, ${ }^{43,44} \mathrm{IL}-1 \beta$ and IL-6 have both pro- and anti-inflammatory properties, but are predominantly involved in the acute-phase immunological response. ${ }^{45-47}$ This may imply that the acute immunological response in IBS patients may not be affected. It should be taken into account that we have measured baseline cytokine levels and not a stimulated cytokine response (e.g. by LPS). Therefore, a presumed role of the measured cytokines in the IBS pathophysiology should be interpreted with care. The level of CgA was increased in IBS patients compared to $\mathrm{HC}$, as has been observed previously, ${ }^{24}$ and may indicate an altered intestinal neuroendocrine activity in IBS patients. Finally, the antimicrobial peptide HBD2, as well as the SCFAs caproate and valerate, were decreased in IBS patients compared to $\mathrm{HC}$. The interpretation with regard to IBS pathophysiology is challenging, since a decreased production as well as increased breakdown or degradation could underlie the observed differences. Nevertheless, the observed differences in faecal concentrations of these markers between IBS patients and $\mathrm{HC}$ are indicative of altered gut microbial activity and/or host-microbe interaction in IBS patients.

Taken together, we have observed significant but subtle differences between IBS patients and HC for several of the tested markers. Separately, in line with our hypothesis, these markers lacked potential for the discrimination between groups, but when combined had a high predictive value with a good sensitivity (88.1\%) and specificity (86.5\%). The final 8-item biomarker panel consists of markers related to several gut-health domains: immunology, intestinal microbiota and neuroendocrine activity.

The current findings demonstrate that it is possible to identify the presence of a functional gut disorder (i.e. IBS) based on a non-invasive biomarker panel even with only subtle changes per single maker between health and disease status. As most clinicians (>70\%) do not solely use the Rome III criteria and frequently rely on additional (invasive) investigations to make a positive diagnosis of IBS, ${ }^{7}$ a non-invasive biomarker panel could aid the diagnostic process of IBS in clinical practice. The identification of the current 'multi-gut health domain' panel of faecal and plasma markers is an important step in the development of reliable biomarkers for IBS. However, before implementation in daily clinical practice, further research is needed, especially to assess the value of the biomarker panel for the discrimination between IBS and organic GI disorders, such as inflammatory bowel disease, and its potential to monitor disease activity and symptoms over time. 
Symptom reduction is the primary goal of therapeutic interventions in IBS. Symptom assessment, mostly by retrospective paper surveys, is challenging and is subject to recall bias and fake compliance. ${ }^{48}$ Intervention trials in IBS patients using symptom scores as main outcomes are hampered by a high placebo response. ${ }^{49}$ The identified biomarker panel was found to correlate moderately though significantly $(r=0.59$, $\mathrm{p}<0.001$ ) with $\mathrm{GI}$ symptom severity in the IBS cohort. This indicates a possible potential of the biomarker panel to quantify responsiveness to treatments in addition to symptom scores, in clinical trials. However, this hypothesis needs to be tested further.

IBS is a highly prevalent disorder among the general population, but difficult to identify in subjects not seeking medical attention. Furthermore, a large part of subjects with IBS symptoms does not seek medical attention but try to improve their well-being by selftreatment. The evaluation of potential beneficial effects of interventions such as food products, supplementals or life style factors on GI health in the general population is challenging, since at group level GI symptom severity is low. Alternatively, a biomarker panel next to Gl symptom questionnaires may have added value to quantify improvements by a specific intervention in the general population. Since our biomarker set is based on multiple gut health domains and correlated significantly with symptoms in IBS patients, we hypothesized that it would also be applicable in the general population. As expected, the majority of subjects form the general population reported to have no or hardly Gl symptoms, resulting in low overall mean symptom scores. As proof of principle, subjects with a 7-day mean score of 1.5 or higher for abdominal pain and discomfort were selected. In this study subgroup, also a significant correlation ( $r=0.51, p=0.003$ ) between the 8 -item biomarker panel and $\mathrm{Gl}$ symptom scores was observed. This may indicate a potential application of the current non-invasive 8-item biomarker panel in research focusing on gut health and possible interventions thereof in the general population. As the biomarker panel reflects various domains of gut health and distinguishes patients with a functional GI disorder from healthy subjects, it may be a first step in the development of a biomarker (set) for healthy gut function.

The current 8-item biomarker panel was identified in a subset of subjects of the Maastricht IBS cohort. Although external validation in a second independent clinical IBS cohort is needed, we validated the biomarker panel in the pre-assigned independent validation set of IBS patients and controls of the Maastricht IBS cohort. These subjects were not used in the biomarker panel discovery step. The LifeLines DEEP cohort was a large population based cohort in which biosamples and symptom scores were collected. This cohort was not included in the current study as an external validation cohort, but to test the potency of the biomarker panel to pick up the presence of gastro-intestinal symptoms in the general population. 


\section{Conclusion}

A novel, non-invasive, 'multi-gut health domain' biomarker panel, with a high predictive value for the discrimination between IBS patients and healthy controls, has been identified and validated. Furthermore, a moderate and highly significant correlation was observed between the biomarker panel and GI symptom severity in a clinical IBS cohort as well as in a general population cohort. Although, further research is needed to test the applicability of the 8-item biomarker panel in daily clinical practice, these findings are an important step towards biomarker based diagnosis of IBS. The biomarker panel has potential to assess responsiveness in IBS therapy trials or of interventions aiming to improve gut health in the general population. 


\section{References}

1. Bischoff SC. 'Gut health': a new objective in medicine? BMC Med. 2011;9:24.

2. Canavan C, West J, Card T. The epidemiology of irritable bowel syndrome. Clin Epidemiol. 2014;6:71-80.

3. Magro F, Langner C, Driessen A, Ensari A, Geboes K, Mantzaris GJ, et al. European consensus on the histopathology of inflammatory bowel disease. J Crohns Colitis. 2013;7:827-51.

4. Hill P, Austin A, Forsyth J, Holmes G. British Society of Gastroenterology guidelines on the diagnosis and management of coeliac disease. Gut. 2015;64:691-2

5. Corsetti M, Van Oudenhove L, Tack J. The quest for biomarkers in IBS-where should it lead us? Neurogastroenterol Motil 2014;26:1669-76.

6. Camilleri M. Review article: biomarkers and personalised therapy in functional lower gastrointestinal disorders. Aliment Pharmacol Ther. 2015;42:818-28.

7. Spiegel BM, Farid M, Esrailian E, Talley J, Chang L. Is irritable bowel syndrome a diagnosis of exclusion?: a survey of primary care providers, gastroenterologists, and IBS experts. Am J Gastroenterol. 2010; 105:848-58.

8. Sood R, Ford AC. Combining biomarkers in irritable bowel syndrome: A forward step towards making a positive diagnosis and directing therapy? Gastroenterology. 2015;148:1471-3.

9. Lembo AJ, Neri B, Tolley J, Barken D, Carroll S, Pan H. Use of serum biomarkers in a diagnostic test for irritable bowel syndrome. Aliment Pharmacol Ther. 2009;29:834-42.

10. Jones MP, Chey WD, Singh S, Gong H, Shringarpure R, Hoe N, et al. A biomarker panel and psychological morbidity differentiates the irritable bowel syndrome from health and provides novel pathophysiological leads. Aliment Pharmacol Ther. 2014;39:426-37.

11. Camilleri M. Peripheral mechanisms in irritable bowel syndrome. N Engla J Med. 2012;367:1626-35.

12. Mujagic Z, Ludidi S, Keszthelyi D, Hesselink MA, Kruimel JW, Lenaerts K, et al. Small intestinal permeability is increased in diarrhoea predominant IBS, while alterations in gastroduodenal permeability in all IBS subtypes are largely attributable to confounders. Aliment Pharmacol Ther. 2014;40:288-97.

13. Ludidi S, Mujagic Z, Jonkers D, Keszthelyi D, Hesselink M, Kruimel J, et al. Markers for visceral hypersensitivity in patients with irritable bowel syndrome. Neurogastroenterol Motil 2014;26:1104-11.

14. Tigchelaar EF, Zhernakova A, Dekens JA, Hermes G, Baranska A, Mujagic Z, et al. Cohort profile: LifeLines DEEP, a prospective, general population cohort study in the northern Netherlands: study design and baseline characteristics. BMJ Open. 2015;5:e006772.

15. Stolk RP, Rosmalen JG, Postma DS, de Boer RA, Navis G, Slaets JP, et al. Universal risk factors for multifactorial diseases: LifeLines: a three-generation population-based study. Eur J Epidemiol. 2008; 23:67-74.

16. Crenn P, Messing B, Cynober L. Citrulline as a biomarker of intestinal failure due to enterocyte mass reduction. Clin Nutr. 2008;27:328-39.

17. Windmueller HG, Spaeth AE. Source and fate of circulating citrulline. Am J Physiol. 1981;241:E473-80.

18. van Vliet MJ, Tissing WJ, Rings EH, Koetse HA, Stellaard F, Kamps WA, et al. Citrulline as a marker for chemotherapy induced mucosal barrier injury in pediatric patients. Pediatr Blood Cancer. 2009;53: 1188-94.

19. Lutgens LC, Blijlevens NM, Deutz NE, Donnelly JP, Lambin P, de Pauw BE. Monitoring myeloablative therapy-induced small bowel toxicity by serum citrulline concentration: a comparison with sugar permeability tests. Cancer. 2005;103:191-9.

20. Bashashati M, Rezaei N, Shafieyoun A, McKernan DP, Chang L, Ohman L, et al. Cytokine imbalance in irritable bowel syndrome: a systematic review and meta-analysis. Neurogastroenterol Motil 2014;26:1036-48.

21. O'Mahony L, McCarthy J, Kelly P, Hurley G, Luo F, Chen K, et al. Lactobacillus and bifidobacterium in irritable bowel syndrome: symptom responses and relationship to cytokine profiles. Gastroenterology. 2005;128:541-51.

22. Wassell J, Dolwani S, Metzner M, Losty H, Hawthorne A. Faecal calprotectin: a new marker for Crohn's disease? Ann Clin Biochem. 2004;41:230-2.

23. Dolwani S, Metzner M, Wassell JJ, Yong A, Hawthorne AB. Diagnostic accuracy of faecal calprotectin estimation in prediction of abnormal small bowel radiology. Aliment Pharmacol Ther. 2004;20:615-21. 
24. Ohman L, Stridsberg M, Isaksson S, Jerlstad P, Simren M. Altered levels of fecal chromogranins and secretogranins in IBS: relevance for pathophysiology and symptoms? Am J Gastroenterol. 2012;107: 440-7.

25. Vora P, Youdim A, Thomas LS, Fukata M, Tesfay SY, Lukasek K, et al. Beta-defensin-2 expression is regulated by TLR signaling in intestinal epithelial cells. J Immunol. 2004;173:5398-405.

26. Langhorst J, Junge A, Rueffer A, Wehkamp J, Foell D, Michalsen A, et al. Elevated human beta-defensin-2 levels indicate an activation of the innate immune system in patients with irritable bowel syndrome. Am J Gastroenterol. 2009;104:404-10.

27. Hamer HM, Jonkers D, Venema K, Vanhoutvin S, Troost FJ, Brummer RJ. Review article: the role of butyrate on colonic function. Aliment Pharmacol Ther. 2008;27:104-19.

28. Tana C, Umesaki Y, Imaoka A, Handa T, Kanazawa M, Fukudo S. Altered profiles of intestinal microbiota and organic acids may be the origin of symptoms in irritable bowel syndrome. Neurogastroenterol Motil 2010;22:512-9, e114-5.

29. Facer P, Bishop AE, Lloyd RV, Wilson BS, Hennessy RJ, Polak JM. Chromogranin: a newly recognized marker for endocrine cells of the human gastrointestinal tract. Gastroenterology. 1985;89:1366-73.

30. Usellini L, Finzi G, Riva C, Capella C, Mochizuki T, Yanaihara C, et al. Ultrastructural identification of human secretin cells by the immunogold technique. Their costorage of chromogranin $A$ and serotonin. Histochemistry. 1990;94:113-20.

31. El-Salhy M, Lomholt-Beck B, Hausken T. Chromogranin A as a possible tool in the diagnosis of irritable bowel syndrome. Scand J Gastroenterol. 2010;45:1435-9.

32. Garcia-Villalba R, Gimenez-Bastida JA, Garcia-Conesa MT, Tomas-Barberan FA, Carlos Espin J, Larrosa M. Alternative method for gas chromatography-mass spectrometry analysis of short-chain fatty acids in faecal samples. J Sep Sci. 2012;35:1906-13.

33. Farzi A, Reichmann F, Meinitzer A, Mayerhofer R, Jain P, Hassan AM, et al. Synergistic effects of NOD1 or NOD2 and TLR4 activation on mouse sickness behavior in relation to immune and brain activity markers. Brain Behav Immun. 2015;44:106-20.

34. Whelan RA, Rausch S, Ebner F, Gunzel D, Richter JF, Hering NA, et al. A transgenic probiotic secreting a parasite immunomodulator for site-directed treatment of gut inflammation. Mol Ther. 2014;22: 1730-40.

35. Benjamini Y, Hochberg Y. Controlling the False Discovery Rate - a Practical and Powerful Approach to Multiple Testing. J Roy Stat Soc B Met. 1995;57:289-300.

36. Schafer JL. Multiple imputation: a primer. Stat Methods Med Res 1999;8:3-15.

37. Wold S, Sjostrom M, Eriksson L. PLS-regression: a basic tool of chemometrics. Chemometr Intell Lab. 2001;58:109-30.

38. Guyon I. A Scaling Law for the Validation-Set Training-Set Size Ratio. AT \& T Bell Laboratories. 1997.

39. Anderssen E, Dyrstad K, Westad F, Martens H. Reducing over-optimism in variable selection by crossmodel validation. Chemometr Intell Lab. 2006;84:69-74.

40. Gidskehaug L, Anderssen E, Alsberg BK. Cross model validated feature selection based on gene clusters. Chemometr Intell Lab. 2006;84:172-6.

41. Esbensen KH, Geladi P. Principles of Proper Validation: use and abuse of re-sampling for validation. J Chemometr. 2010;24:168-87.

42. Hardoon DR, Szedmak S, Shawe-Taylor J. Canonical correlation analysis: An overview with application to learning methods. Neural Comput. 2004;16:2639-64.

43. Liu ZJ, Geboes K, Heremans H, Overbergh L, Mathieu C, Rutgeerts P, et al. Role of interleukin-12 in the induction of mucosal inflammation and abrogation of regulatory $\mathrm{T}$ cell function in chronic experimental colitis. Eur J Immunol. 2001;31:1550-60.

44. Vignali DAA, Kuchroo VK. IL-12 family cytokines: immunological playmakers. Nat Immunol. 2012;13: 722-8.

45. Zheng $\mathrm{H}$, Fletcher D, Kozak W, Jiang MH, Hofmann KJ, Conn CA, et al. Resistance to Fever Induction and Impaired Acute-Phase Response in Interleukin-1-Beta-Deficient Mice. Immunity. 1995;3:9-19.

46. Dinarello CA. Immunological and Inflammatory Functions of the Interleukin-1 Family. Annu Rev Immunol. 2009;27:519-50.

47. Scheller J, Chalaris A, Schmidt-Arras D, Rose-John S. The pro- and anti-inflammatory properties of the cytokine interleukin-6. Biochim Biophys Acta. 2011;1813:878-88. 
48. Mujagic Z, Keszthelyi D, Aziz Q, Reinisch W, Quetglas EG, De Leonardis F, et al. Systematic review: instruments to assess abdominal pain in irritable bowel syndrome. Aliment Pharmacol Ther. 2015; 42:1064-81..

49. Ford AC, Moayyedi P. Meta-analysis: factors affecting placebo response rate in the irritable bowel syndrome. Aliment Pharmacol Ther. 2010;32:144-58. 


\section{Chapter 11}

\section{Novel breath biomarkers for IBS;}

\section{a metabolomic approach}

Volatile Organic Compounds in breath as marker for Irritable Bowel Syndrome:

a metabolomic approach

Agnieszka Baranska*, Zlatan Mujagic*, Agnieszka Smolinska, Jan W Dallinga, Daisy DMA Jonkers, Ettje F Tigchelaar, Jackie Dekens, Alexandra Zhernakova, Thomas Ludwig, Ad AM Masclee, Cisca Wijmenga, Frederik J van Schooten

* Authors contributed equally to the manuscript

Submitted 


\section{Abstract}

\section{Background \& Aims}

The diagnosis of Irritable Bowel Syndrome (IBS) is challenging because of its heterogeneity and multifactorial pathophysiology. No reliable biomarkers have been identified so far. We investigated a novel application of breath analysis with the goal to distinguish IBS patients from healthy controls based on a set of volatile organic compounds (VOCs). Additionally, the VOC-biomarker set was correlated to selfreported gastrointestinal (GI) symptoms of subjects of the Maastricht IBS clinical cohort (MIBS) and of a general population cohort, LifeLines DEEP (LL DEEP).

\section{Methods}

Breath samples were collected from 170 IBS patients and 153 healthy controls in MIBS and from 1,307 participants in LL DEEP. Multivariate statistics were used to identify the most discriminatory set of VOCs and to find associations between VOCs and GI symptoms in both cohorts.

\section{Results}

A set of 16 VOCs correctly predicted $89.4 \%$ of the IBS patients and $73.3 \%$ of the healthy controls ( $A \cup C=0.83$ ). The VOC-biomarker set correlated significantly with a set of $\mathrm{GI}$ symptoms in the MIBS $(r=0.55, p=0.0003)$ and LL DEEP cohorts $(r=0.54, p=0.0004)$. A Kruskal-Wallis test showed no influence from possible confounding factors in distinguishing IBS patients from healthy controls.

\section{Conclusion}

A set of breath-based biomarkers (16 VOCs) that distinguishes IBS patients from healthy controls was identified. The VOC-biomarker set correlates significantly with GI symptoms in two independent cohorts. We demonstrate the potential use of breath analysis in the diagnosis and monitoring of IBS, and a possible application of VOCanalyses in a general population cohort. 


\section{Introduction}

Irritable Bowel Syndrome (IBS) is a functional gastrointestinal (GI) disorder characterized by abdominal pain and altered bowel habits. IBS has a high prevalence of up to $15 \%$ among the general population in Western countries. ${ }^{1}$ It is a heterogeneous disorder with a multifactorial pathophysiology including alterations in intestinal motility, visceral perception, intestinal microbiota, barrier function, low-grade immune activation, and psychological factors. ${ }^{2}$ Until now, the diagnosis of IBS is symptom-based, using the Rome III criteria, ${ }^{3}$ but objective and validated biomarkers are lacking. ${ }^{4}$ When available, the ideal biological marker should differentiate IBS patients from healthy individuals with occasional GI symptoms and from patients with organic GI diseases, such as inflammatory bowel diseases (IBD). Biomarkers for IBS should not only provide insight into the pathophysiological mechanisms that generate symptoms but also be helpful in quantifying efficacy of therapeutic interventions in IBS.

Searches for individual biomarkers in IBS have failed so far, mainly because of the inability to capture the complexity and heterogeneity of IBS and the variation in symptoms. Therefore, we propose a more holistic approach. Using multiple characteristics and factors simultaneously, we intend to cover the full heterogenic spectrum of IBS. Analysis of exhaled breath is a promising and non-invasive tool in disease diagnosis and monitoring. ${ }^{5-9}$ Hundreds of volatile organic compounds (VOCs) are present in human exhaled breath, of which the majority is comparable between individuals. Some compounds however, differ significantly according to metabolic differences and may indicate certain states of health or disease. Volatiles originate from several sources including inflamed tissue, normal and altered metabolic pathways or from microbiota metabolism in the body. Upon formation, VOCs reach the blood-lung barrier through the blood stream and, due to their low solubility, are easily and rapidly excreted via the airways. Recently we have demonstrated the value of VOCs analysis in monitoring disease activity in Crohn's disease. ${ }^{7}$

The aim of the present study was to use VOC analysis of exhaled breath to describe the presence of IBS and to search for correlations between VOCs and GI symptoms in both IBS patients and individuals from a general population cohort. More specifically, our three research aims were:

1. To determine a specific set of VOCs that distinguishes IBS patients from control subjects without GI complaints using the clinical Maastricht IBS cohort (MIBS)

2. To test whether our set of VOCs correlates with GI symptoms in the clinical IBS cohort (MIBS)

3. To test a possible implication of the VOCs biomarker set in a general population cohort, by testing whether our set of VOCs relates to more pronounced GI symptoms reported by subjects in a general population cohort (LL DEEP cohort) 


\section{Materials and methods}

\section{Study participants}

Two cohorts were included into this study. To fulfil previously mentioned aim 1 and 2 we used Maastricht IBS (MIBS) cohort, as it represents a well-defined group of IBS subjects and also controls, free of GI complaints. Aim 3 was accomplished by inclusion of a general population cohort, the LL DEEP cohort.

\section{MIBS cohort}

Breath samples were collected from 170 well-phenotype IBS patients and from 153 healthy volunteers without GI complaints, who participated in a large clinical cohort study (MIBS) on the phenotypic and genotypic characterization of patients with IBS. ${ }^{10}$ IBS patients, with the age ranging from 18 to 75 years, had been included in the cohort between September 2009 and January 2014 via the Gastroenterology-Hepatology outpatient clinic of Maastricht University Medical Centre+ (MUMC+), a secondary and tertiary referral centre, and via general practitioners (GPs). All patients were diagnosed according to the ROME III criteria and were examined by a gastroenterologist or GP. GI endoscopy, abdominal imaging and/or blood and faecal analyses were performed to exclude organic disease when indicated. Patients with a history of abdominal surgery, apart from appendectomy, laparoscopic cholecystectomy, or hysterectomy, were excluded. Age and gender matched healthy controls were enrolled via public advertisements. The controls were enrolled via public advertisements and were subjected to a detailed medical interview to exclude the presence of $\mathrm{Gl}$ disorders and current or previous GI complaints or symptoms.

All participants filled in questionnaires on demographics and life style. Their GI symptoms were assessed by an end-of-day GI symptom diary recorded for the period of two weeks. ${ }^{10}$ Mean 14-day scores per symptom were used for the analyses. Data on medication use and medical history were self-reported and cross-checked with hospital medical records.

\section{LL DEEP cohort}

Adult participants were enrolled from LifeLines, a general population cohort in the Netherlands. ${ }^{11}$ In a subgroup of 1307 non-selected participants (LL DEEP: Lifelines Detailed Extensive Examination of Participants) exhaled breath samples were collected, along with a 7-day GI symptom diary, identical to the 2-week GI symptom diary used in the MIBS cohort. Information on clinical diagnoses of IBS was not available for this general population cohort. The LL DEEP study design has been described in detail elsewhere. $^{12}$ 


\section{Sample collection and analysis}

Each participant inflated a 3 L Tedlar Bag (SKC Ltd, Dorset, UK) with no specific instructions on the method of exhaling. For each cohort, all samples were collected in the same room. To avoid content alterations, the VOC contents of each bag was captured within an hour in stainless-steel two-bed sorption tubes, filled with carbograph 1TD/Carbo-pack X (Markes International, Llantrisant, UK) and stored at room temperature until analysis. ${ }^{13}$ Breath samples were analysed by thermal desorption-gas chromatography combined with time-of-flight mass spectrometry (TDGC-tof-MS), as previously described. ${ }^{13}$

\section{Sample analysis}

\section{Baseline characteristics}

Raw GC-tof-MS data were pre-processed to diminish the effects of noise, column bleeding, and chromatographic drift, as described previously. ${ }^{14}$

The differences in the baseline characteristics were tested using independent samples T-test (continuous, normally distributed data) and Pearson Chi-squared test (dichotomous data).

\section{Aim 1: Search for VOCs biomarker in MIBS}

Random Forest (RF) was used to determine a set of volatile organic compounds that best separate IBS patients from controls in the MIBS cohort. ${ }^{14}$ First, chromatograms of IBS patients and the healthy control group were divided into a training $( \pm 80 \%$ of the total population) and a validation set $( \pm 20 \%$ of the population) using the Duplex algorithm. ${ }^{15}$ We included 123 subjects of each group in a training set to find discriminatory VOCs and build a RF model. The remaining 47 IBS patients and 30 controls were used as an internal validation set to test the accuracy of the selected VOCs. The receiver operating characteristic (ROC) curve was used to demonstrate the performance of the RF classification model for the validation set. For each sample, RF delivered a probability of it belonging to the IBS or control group. These probabilities were used as a range of cut-off values to calculate the sensitivity and specificity presented in a ROC curve.

For visualization, we performed Principal Component Analysis (PCA) on the proximity matrix obtained from the RF model built on the set of discriminatory VOCs. ${ }^{14}$

\section{Influence of subjects' characteristics and importance of individual VOCS}

Potential contribution of the external factors defined by subjects' characteristics, to the separation seen due to the set of 16 VOCs, was checked by interpreting a PCA score plot. To see if any of the individual VOCs from the chosen set was statistically 
influenced by external factors, we combined a Kruskal-Wallis test with a BenjaminiHochberg post-hoc correction for multiple testing. ${ }^{16}$

Correlation between the VOC set and symptoms in MIBS (Aim 2) and LL DEEP population (Aim 3)

The linear correlations between the most discriminatory set of VOCs and a set of symptoms including all items of the GI symptom diary were tested in the MIBS and in the general population LL-DEEP cohort, using canonical correlation analysis (CCA), ${ }^{17}$ which is an extension of bi-variate correlation. CCA calculates a linear correlation that best explains the variability both within and between the biomarker panel and the measured GI symptoms.

\section{VOCs identification}

The set of VOCs was tentatively identified by means of spectrum recognition, using the NIST (National Institute of Standards and Technology) library in combination with expert interpretation.

\section{Results}

\section{Baseline characteristics}

The baseline characteristic of MIBS cohort is given in Tables $11.1 \mathrm{~A}$ and $11.1 \mathrm{~B}$. Although, IBS patients and healthy controls exhibit several differences in life style and supplements and drugs intake, no differences were observed in demographic characteristic and diet (Table 11.1A). As expected IBS patients reveal much higher GI symptoms in comparison to healthy controls (Table 11.1B). The largest difference between IBS and healthy controls was observed in abdominal pain.

Table 11.1A Demographics and lifestyle of the MIBS cohort

\begin{tabular}{lcc}
\hline & $\begin{array}{c}\text { Healthy controls } \\
(\mathrm{n}=153)\end{array}$ & $\begin{array}{c}\text { IBS patients } \\
(\mathrm{n}=170)\end{array}$ \\
\hline Demographic characteristics & & $42.9 \pm 16.7$ \\
Age (mean years \pm SD) & $43.7 \pm 19.1$ & 72.4 \\
Female sex (\%) & 63.1 & $24.9 \pm 4.4$ \\
BMI (mean kg/m ${ }^{2} \pm$ SD) & $24.0 \pm 4.0$ & \\
Lifestyle parameters & & $25.3^{* * *}$ \\
Current smoker (\%) & 7.6 & $51.3^{* * *}$ \\
Current or previous smoker (\%) & 38.6 & $63.0^{* * *}$ \\
Alcohol intake: $>0$ units/week (\%) & 81.1 & \\
\hline
\end{tabular}


(continued)

\begin{tabular}{lcc}
\hline & $\begin{array}{c}\text { Healthy controls } \\
(\mathrm{n}=153)\end{array}$ & $\begin{array}{c}\text { IBS patients } \\
(\mathrm{n}=170)\end{array}$ \\
\hline Use of medication, 2 weeks prior to participation in study & & $78.7^{* * *}$ \\
Use of any medication (\%) & 41.6 & $20.2^{* * *}$ \\
PPI (\%) & 2.0 & $9.2^{*}$ \\
SSRI (\%) & 2.7 & $3.7^{*}$ \\
Prokinetic drugs (\%) & 0.0 & $17.8^{* * *}$ \\
Laxatives (\%) & 0.6 & $4.3^{*}$ \\
Antidiarrheal drugs (\%) & 0.0 & 9.8 \\
NSAID (\%) & 10.1 & 16.6 \\
Antihypertensive drugs (\%) & 12.8 & 9.8 \\
Statins (\%) & 5.4 & 15.4 \\
Dietary habits & & $44.6^{* *}$ \\
Current use of any diet (\%) & 9.4 & 25.3 \\
Use of dietary supplements (\%) & & \\
\hline
\end{tabular}

PPI: proton pump inhibitors, SSRI: selective serotonin reuptake inhibitors, NSAID: non-steroid antiinflammatory drugs. Differences between the two groups were tested with the independent samples T-test (continuous data) and Pearson Chi-squared test (dichotomous data). ${ }^{*} p<0.05 ;{ }^{* *} p<0.01 ;{ }^{* *} p<0.001$ vs. healthy control group

Table 11.1B Gl symptom scores of the MIBS cohort

\begin{tabular}{lcc}
\hline & $\begin{array}{c}\text { Healthy controls } \\
(\mathrm{n}=153)\end{array}$ & $\begin{array}{c}\text { IBS patients } \\
(\mathrm{n}=170)\end{array}$ \\
\hline GI symptom scores, by end-of-day diary (14-day mean $\pm \mathrm{SD}), 1$-to-5 point scale & $2.2 \pm 0.8^{*}$ \\
Abdominal pain & $1.1 \pm 0.2$ & $2.4 \pm 0.8^{*}$ \\
Abdominal discomfort & $1.1 \pm 0.2$ & $2.2 \pm 0.9^{*}$ \\
Bloating & $1.1 \pm 0.2$ & $1.6 \pm 0.7^{*}$ \\
Belching & $1.1 \pm 0.3$ & $1.6 \pm 0.8^{*}$ \\
Nausea & $1.0 \pm 0.1$ & $2.2 \pm 0.8^{*}$ \\
Flatulence & $1.3 \pm 0.5$ & $1.4 \pm 0.6^{*}$ \\
Constipation & $1.1 \pm 0.2$ & $1.5 \pm 0.7^{*}$ \\
Diarrhoea & $1.0 \pm 0.1$ & \\
\hline
\end{tabular}

The end-of-day diary scores are based on a 1-to-5 point scale, 14 day means are presented. Differences between the two groups were tested with the independent samples T-test. ${ }^{*} p<0.001$ vs. healthy control group

Table 11.2 shows the characteristics of LL DEEP cohort. The demographic features of LL DEEP cohort resemble both groups of MIBS. As said previously LL DEEP is a general population cohort. Thus, it is not surprising that the Gl symptoms shown in Table 11.2 are more comparable to healthy controls from MIBS.

\section{Preparation of MIBS and LL DEEP data}

In the MIBS cohort (170 IBS patients and 153 healthy controls), we obtained 323 chromatograms by GC-tof-MS with a total of 587 different VOCs, each compound present in at least $20 \%$ of either IBS or healthy controls.

For LL DEEP, a total of 1307 chromatograms were measured, showing 3283 VOCs in total. No reduction in VOCs was applied at this step as we performed a reverse search for the established set of VOCs. 
Table 11.2 Baseline characteristics of the LL DEEP cohort

\begin{tabular}{lc}
\hline Demographical \& lifestyle characteristics $(\mathrm{n}=1307)$ & \\
\hline Age (mean years $\pm \mathrm{SD})$ & $45.4 \pm 13.5$ \\
Female sex $(\%)$ & 58.2 \\
BMI (mean kg/m2 $\pm \mathrm{SD})$ & $25.5 \pm 4.4$ \\
Current smoker $(\%)$ & 19.6 \\
Gl symptom scores by end-of-day diary (7-day mean \pm SD), 1-to-5 point scale & \\
Abdominal pain & $1.2 \pm 0.4$ \\
Abdominal discomfort & $1.3 \pm 0.5$ \\
Bloating & $1.5 \pm 0.6$ \\
Belching & $1.1 \pm 0.4$ \\
Nausea & $1.1 \pm 0.3$ \\
Flatulence & $1.8 \pm 0.7$ \\
Constipation & $1.2 \pm 0.4$ \\
Diarrhoea & $1.1 \pm 0.3$ \\
\hline
\end{tabular}

The end-of-day diary scores are based on a 1-to-5 point scale, 7 day means are presented.

\section{Data analysis}

\section{Aim 1: Search for VOC biomarkers in MIBS}

RF identified a group of 16 VOCs with best discriminatory power between IBS patients and healthy controls in the training set. They were then tested on the internal validation set of 47 breath-o-grams of IBS patients and 30 of healthy controls, resulting in a sensitivity of $89.4 \%$ and a specificity of $73.3 \%$ with a positive predictive value of $84 \%$, and a negative predictive value of $81.5 \%$. The corresponding ROC curve, with the area under the curve (AUC) of 0.83, is shown in Figure 11.1.

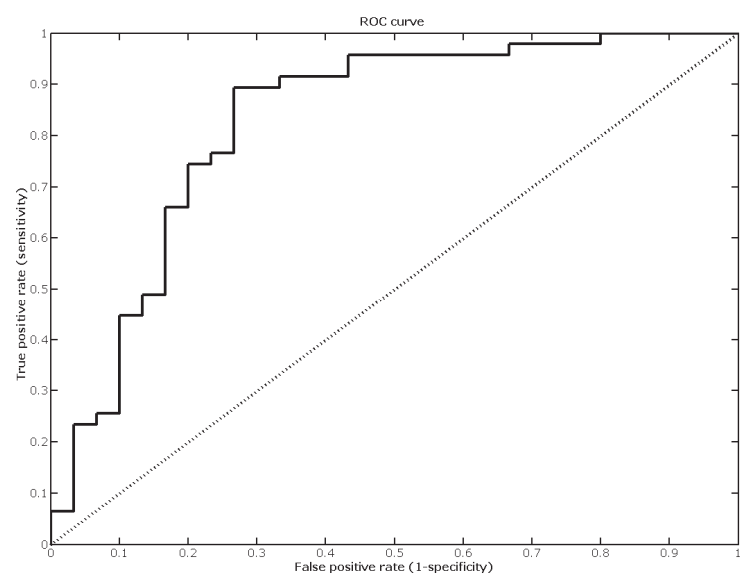

Figure 11.1 ROC curve performed on the validation set of samples, with area under the curve $(A \cup C)=0.83$. 
PCA was then performed on the proximity matrix obtained from the RF model built on the 16 discriminatory VOCs. The corresponding score plot (Figure 11.2) shows the distributions of the breath-o-grams in the training set (squares for IBS and diamonds for healthy controls) and in the validation set (stars for IBS and circles for healthy controls). There is a visible separation between the IBS patient and healthy control breath-ograms in training set but more importantly in validation set. The partial overlap between the groups indicates that some IBS patients have VOC profiles more similar to healthy controls and vice versa, but these could not be related to either medical history or baseline clinical characteristics. The overall variation was less in the healthy controls than in the IBS group.

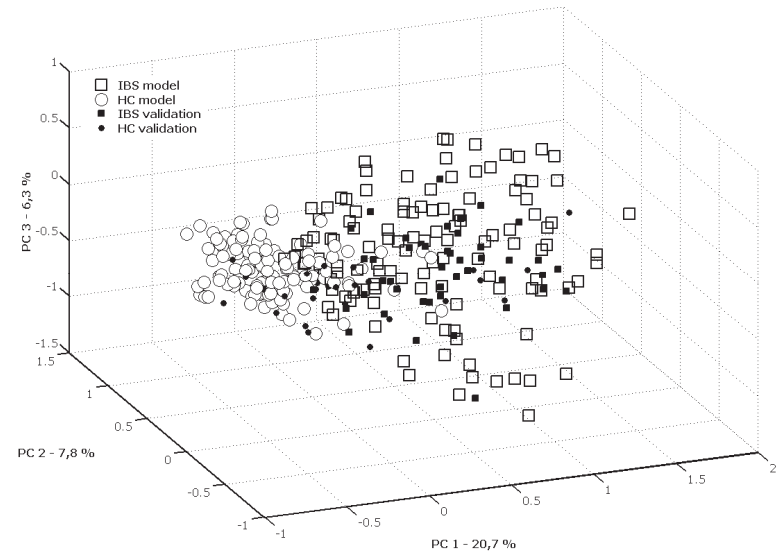

Figure 11.2 3D PCA score plot, on a proximity matrix obtained from the Random Forest model built on 16 selected VOCs. Each point is a single chromatogram.

\section{Influence of subjects' characteristics}

To ensure that the separation seen between the healthy controls and IBS patients (see Figure 11.2) was not related to other factors we displayed all available information on the same score plot. No associations or even trends were observed in relation to age, sex, smoking habits, medication, use of dietary supplements, specific diet, or the presence of depressive and/or anxiety symptoms. PCA score plots indicating the relation between VOCs and medication use or diet, together with the reference plots indicating the distribution of IBS patients and healthy controls, are shown in Figure 11.3. All eight external factors are shown in Supplementary Figure S11.1.

Each selected VOC was also tested individually with the Kruskal-Wallis test combined with the Benjamini-Hochberg post-hoc correction for multiple testing. No significant differences were observed between their levels and the studied factors. The absence of the influence of these possible cofounders increases our confidence in the reliability of this set of 16 VOCs as a potential IBS biomarker. 

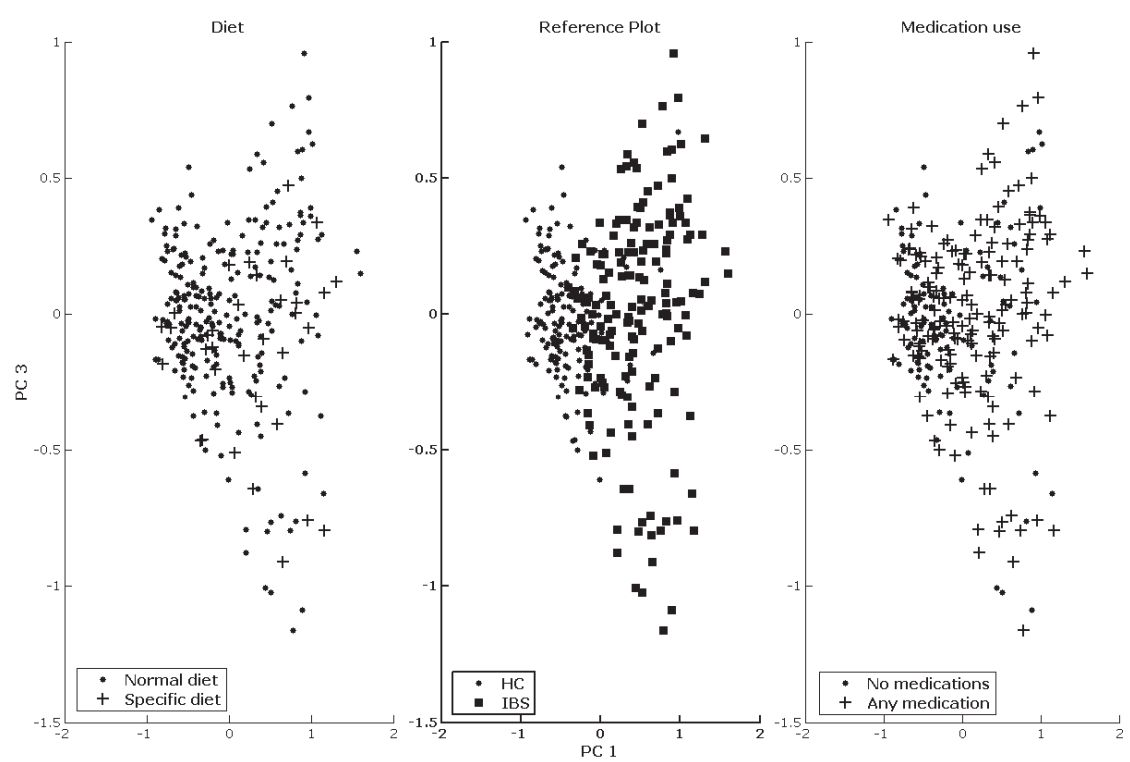

Figure 11.3 PCA score plots, defined by PC1 and PC3, on a proximity matrix obtained from the Random Forest model built on 16 selected VOCs. Each point is a single chromatogram, with points shaped by external factors.

Importance of individual VOCs in the discriminative set

Even though it is the set of all 16 VOCs together that yields that high discriminative power (sensitivity of $89.4 \%$ and a specificity of $73.3 \%$ ), we also analysed the relative importance of the 16 discriminatory VOCs when considered individually for comparison between the IBS and control groups using the Kruskal-Wallis test. Twelve out of 16 compounds were statistically significant, with a p-value $\leq 0.05$ (Figure 11.4). That additionally highlights that the power of the biomarker comes from the set, not individual compounds. 


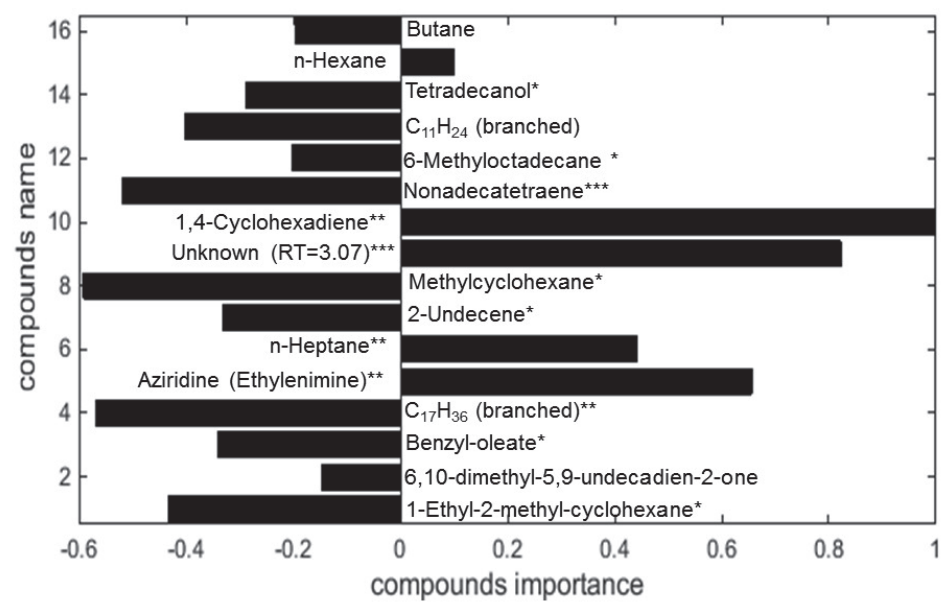

Figure 11.3 List of 16 VOCs found to discriminate between IBS group and healthy control group in MIBS. Negative compound importance indicates a decrease and positive an increase in VOC concentration in the IBS group compared to the control group. p-values (univariate test) for individual VOC significance are given. RT = retention time

\section{Correlation between VOC set and symptoms in:}

\section{MIBS cohort (Aim 2)}

The correlation was checked between the discriminatory set of VOCs and the set of GI symptom scores which are presented in Table 11.1B. We found a significant correlation of $r=0.55(p=0.0003)$ between the set of 16 VOCs and the set of following symptom scores: abdominal pain, discomfort, flatulence and belching, which were assessed by the 14-day end-of-day Gl symptom diary. No correlations were found between the VOCs and the other GI symptom scores presented in Table 11.1B.

\section{Application of the biomarker panel in the general population LL DEEP cohort (Aim 3)}

Similarly, we investigated the correlation between the discriminatory set of VOCs and the set of eight available symptoms from the 7-day end-of-day GI symptom diary available for the LL DEEP cohort (Table 11.2). Here, the mean scores for abdominal pain and discomfort above 1.5 (on a 1-to-5 point scale) in the 7-day mean scores of the GI symptom diary, determined the subset of data used in the correlation analysis, in total 239 participants. A mean score of 1.5 or higher indicates the subject had at least 2 days moderately-severe or 4 days slightly- severe Gl symptoms during those 7 days. This cutof value of 1.5 was further supported by data of the MIBS cohort in which $95 \%$ of healthy controls had end-of-day diary mean-scores below 1.5 for these Gl symptoms. 
In the LL DEEP cohort the 7-day mean values for abdominal pain, flatulence, belching, bloating, diarrhoea and nausea were found to be highly and significantly correlated with our set of VOCs $(r=0.54, p=0.0004)$

\section{Discussion}

In this paper we have demonstrated a possible use of VOCs in exhaled breath analysis for diagnosis and monitoring of IBS patients. We showed that non-invasive breath analysis is suitable to distinguish IBS patients from healthy controls. The first aim of our study was to apply a holistic metabolomics approach by analysing a large number of exhaled VOCs and searching for differences in patterns between IBS patients and healthy controls. A pattern of 16 VOCs was identified to be most discriminative for the presence or absence of IBS in a large group of well phenotyped IBS patients and healthy controls (MIBS). The proposed IBS breath-based biomarker set has a sensitivity of $89.4 \%$ and specificity of $73.3 \%$. We have clearly shown that the selected VOCs (as a set and separately) are related only to the presence of IBS and are not affected by any of the possible confounders we investigated.

To our knowledge, this is the first time that a set of VOCs in exhaled air was able to predict the presence of a common functional gastrointestinal disorder. This can be considered an important first step forward in the design and development of reliable non-invasive biomarkers for IBS.

As the diagnosis of IBS is based on symptoms, our second aim was to test whether our VOC biomarker set would also correlate with severity of $\mathrm{Gl}$ symptoms. Four GI symptoms showed a significant correlation $(r=0.55, p=0.0003)$ with the set of 16 VOCs in the clinical IBS cohort. This finding could be clinically useful, with the prospect to improve monitoring of the IBS course in intervention studies.

Since functional GI symptoms observed in IBS patients are common in the general population, next we tested for correlations between the set of 16 VOCs and GI symptoms reported in a general population cohort (LL DEEP). This cohort was not included in the current study as an external validation cohort, but to test the potency of the biomarker panel to pick up the presence of gastro-intestinal symptoms in the general population. Although the overall Gl symptom scores were low in the general population, the set of 16 VOCs correlated moderately but significantly with $\mathrm{GI}$ symptoms $(r=0.54, p=0.0004)$. This finding is relevant for several reasons. First, it enables identifying subjects with GI symptoms among the general population. Second, it offers an opportunity to monitor effects of therapeutic interventions in relation to underlying pathophysiological mechanisms. Since the collection of exhaled breath is non-invasive, easy to apply, and requires only a minimal time investment, this method is potentially suitable for use in large cohorts and highly prevalent conditions in the general population. 
Although each VOC can be interpreted and analysed separately, it is the combination of the 16 selected VOCs that yielded the highest discriminatory power between IBS patients and healthy controls. The biological interpretation and background of the compounds is difficult because of the complexity of the underlying metabolic pathways. However, even without the mechanisms being fully understood and explored, our VOCbiomarker set has potential value for diagnostics.

IBS is characterized by low grade chronic inflammation, which results in increased oxidative stress leading to oxidation of larger molecules including lipids (e.g. polyunsaturated fatty acids). ${ }^{18}$ This may give degradation products like hydrocarbons, dienes, aldehydes, lipid peroxides and isoprostanes, which are excreted as VOCs, and subtle changes may be detected in breath. Indeed, the majority of our selected VOCs belong to the (saturated or unsaturated) hydrocarbon family. Some of them, like nhexane, n-heptane and 1,4-cyclohexadiene, are increased in the IBS group, while others, like butane and 2-undecane, are increased in healthy controls. These compounds were all identified earlier as potential oxidative stress markers. ${ }^{19-21}$

Another factor known to contribute to IBS is changes in an altered gut microbial composition. ${ }^{22}$ Products of microbial origin are found in exhaled breath, indicating that systemic alterations of the gut flora may also be one of the factors influencing breath composition. $^{23,24}$ Interestingly, aziridine was found to be increased in breath of IBS patients. This is a parent compound for organic substances containing the aziridine functional group, three-membered heterocyclic containing nitrogen. Aziridine containing compounds were previously detected in faeces but have not been described in exhaled breath before. ${ }^{25}$ Aziridine containing compounds are isolated from the soil bacteria, Streptomyces caespitosus and lavendualeare, which are capable of living in symbiosis with plants, fungi and animals. ${ }^{26}$ Since they belong to the Actinobacteria Phyla, one of the four predominant phyla in the human body, it can be speculated that aziridines may be produced by this or other similar types of gut bacteria. ${ }^{27}$ Antibiotic use is more frequent among IBS patients and certain microbiota strains may overgrow in a post-antibiotic, replenished gut, ${ }^{28}$ leading to different metabolic breath profiles containing for instance, aziridine. Aziridine origin is purely speculative; nevertheless it suggests interesting connections that should be investigated further. It has to be noted that in the IBS cohort included in the present study, the use of antibiotics in two weeks prior to inclusion was rare, and therefore the data were not statistically corrected for this parameter.

Some limitations of our study should be discussed. One may argue that a diagnosis of IBS is based purely on symptoms and thus the need for biomarkers in IBS is limited. Daily practice is quite different, revealing high health care costs for diagnostic processing in IBS. In addition to the Rome III criteria reliable biomarkers could facilitate the diagnostic process of IBS. We initiated a study as first step, to explore whether based on an exhaled metabolomics profile IBS patients can be differentiated from controls. We acknowledge that further validation is needed replicate our data and to 
test the potency of our VOCs biomarker set in a second independent cohort in discriminating between IBS patients and subjects with comparable GI symptoms based on organic causes, such as Inflammatory Bowel Disease or Coeliac Disease. Furthermore, in depth research is needed to investigate the origin of the discriminatory VOCs and their potential link to the pathophysiology of IBS.

\section{Conclusions}

A set of 16 VOCs in exhaled breath can be used to distinguish IBS patients from healthy controls with $89.4 \%$ sensitivity and $73.3 \%$ specificity. We also demonstrated a moderate but significant correlation between sets of GI symptoms and the VOCsbiomarker set for both clinical IBS patients and subjects in a general population cohort. Our findings emphasize the relevance of this VOCs-biomarker set for the diagnostic process and for monitoring therapeutic interventions in IBS patients, but also its potential to be used as a monitoring tool for gastrointestinal health in the general public. 


\section{References}

1. Canavan C, West J, Card T. The epidemiology of irritable bowel syndrome. Clin Epidemiol. 2014;6:71-80.

2. Chey WD, Kurlander J, Eswaran S. Irritable bowel syndrome: a clinical review. JAMA. 2015;313:949-58.

3. Longstreth GF, Thompson WG, Chey WD, Houghton LA, Mearin F, Spiller RC. Functional bowel disorders. Gastroenterology. 2006;130:1480-91.

4. Plavsic I, Hauser G, Tkalcic M, Pletikosic S, Salkic N. Diagnosis of Irritable Bowel Syndrome: Role of Potential Biomarkers. Gastroenterol Res Pract. 2015;2015:490183.

5. de Boer NKH, de Meij TGJ, Oort FA, Ben Larbi I, Mulder CJJ, van Bodegraven AA, et al. The Scent of Colorectal Cancer: Detection by Volatile Organic Compound Analysis. Clinical Gastroenterology and Hepatology. 2014;12:1085-9.

6. Khalid T, Richardson P, Probert CS. The Liver Breath! Breath Volatile Organic Compounds for the Diagnosis of Liver Disease. Clin Gastroenterol Hepatol. 2014;12:524-6.

7. Bodelier AGL, Smolinska A, Baranska A, Dalling JW, Mujagic Z, Vanhees K, et al. Volatile organic compounds in exhaled air as novel marker for disease activity in Crohn's disease; a metabolomic approach. Inflamm Bowel Dis. 2015; in press.

8. Kurada S, Alkhouri N, Fiocchi C, Dweik R, Rieder F. Review article: breath analysis in inflammatory bowel diseases. Aliment Pharmacol Ther. 2015;41:329-41.

9. Arasaradnam RP, Covington JA, Harmston C, Nwokolo CU. Review article: next generation diagnostic modalities in gastroenterology--gas phase volatile compound biomarker detection. Aliment Pharmacol Ther. 2014;39:780-9.

10. Mujagic Z, Ludidi S, Keszthelyi D, Hesselink MA, Kruimel JW, Lenaerts K, et al. Small intestinal permeability is increased in diarrhoea predominant IBS, while alterations in gastroduodenal permeability in all IBS subtypes are largely attributable to confounders. Aliment Pharmacol Ther. 2014;40:288-97.

11. Stolk RP, Rosmalen JG, Postma DS, de Boer RA, Navis G, Slaets JP, et al. Universal risk factors for multifactorial diseases: LifeLines: a three-generation population-based study. Eur J Epidemiol. 2008;23:67-74.

12. Tigchelaar EF, Zhernakova A, Dekens JA, Hermes G, Baranska A, Mujagic Z, et al. Cohort profile: LifeLines $\mathrm{DEEP}$, a prospective, general population cohort study in the northern Netherlands: study design and baseline characteristics. BMJ Open. 2015;5:e006772.

13. Dallinga JW, Smolinska A, van Schooten FJ. Analysis of volatile organic compounds in exhaled breath by gas chromatography-mass spectrometry combined with chemometric analysis. Methods Mol Biol. 2014;1198:251-63.

14. Smolinska A, Hauschild AC, Fijten RR, Dallinga JW, Baumbach J, van Schooten FJ. Current breathomics--a review on data pre-processing techniques and machine learning in metabolomics breath analysis. J Breath Res. 2014;8:027105.

15. Snee RD. Validation of regression models: Methods and examples. Technometrics. 1977;19(4):415-28.

16. Benjamini Y, Hochberg Y. Controlling the False Discovery Rate - a Practical and Powerful Approach to Multiple Testing. Journal of the Royal Statistical Society Series B-Methodological. 1995;57:289-300.

17. Hardoon DR, Szedmak S, Shawe-Taylor J. Canonical correlation analysis: an overview with application to learning methods. Neural Comput. 2004;16:2639-64.

18. Mete R, Tulubas F, Oran M, Yilmaz A, Avci BA, Yildiz K, et al. The role of oxidants and reactive nitrogen species in irritable bowel syndrome: a potential etiological explanation. Med Sci Monit. 2013;19:762-6.

19. Phillips M, Cataneo RN, Cummin AR, Gagliardi AJ, Gleeson K, Greenberg J, et al. Detection of lung cancer with volatile markers in the breath. Chest. 2003;123:2115-23.

20. Poli D, Carbognani P, Corradi M, Goldoni M, Acampa O, Balbi B, et al. Exhaled volatile organic compounds in patients with non-small cell lung cancer: cross sectional and nested short-term follow-up study. Respir Res. 2005;6:71.

21. Chen $X, X u F$, Wang $Y$, Pan $Y$, Lu D, Wang $P$, et al. A study of the volatile organic compounds exhaled by lung cancer cells in vitro for breath diagnosis. Cancer. 2007;110:835-44.

22. Rajilic-Stojanovic M, Jonkers DM, Salonen A, Hanevik K, Raes J, Jalanka J, et al. Intestinal microbiota and diet in IBS: causes, consequences, or epiphenomena? Am J Gastroenterol. 2015;110:278-87. 
23. Turner C, Spanel P, Smith D. A longitudinal study of ammonia, acetone and propanol in the exhaled breath of 30 subjects using selected ion flow tube mass spectrometry, SIFT-MS. Physiol Meas. 2006;27:321-37.

24. Lindinger W, Taucher J, Jordan A, Hansel A, Vogel W. Endogenous production of methanol after the consumption of fruit. Alcohol Clin Exp Res. 1997;21:939-43.

25. de Lacy Costello B, Amann A, Al-Kateb H, Flynn C, Filipiak W, Khalid T, et al. A review of the volatiles from the healthy human body. J Breath Res. 2014;8:014001.

26. Seipke RF, Kaltenpoth M, Hutchings MI. Streptomyces as symbionts: an emerging and widespread theme? Fems Microbiology Reviews. 2012;36:862-76.

27. Johnson $\mathrm{CL}$, Versalovic J. The human microbiome and its potential importance to pediatrics. Pediatrics. 2012;129:950-60.

28. Mendall MA, Kumar D. Antibiotic use, childhood affluence and irritable bowel syndrome (IBS). Eur J Gastroenterol Hepatol. 1998;10:59-62. 


\section{Supplementary figure}
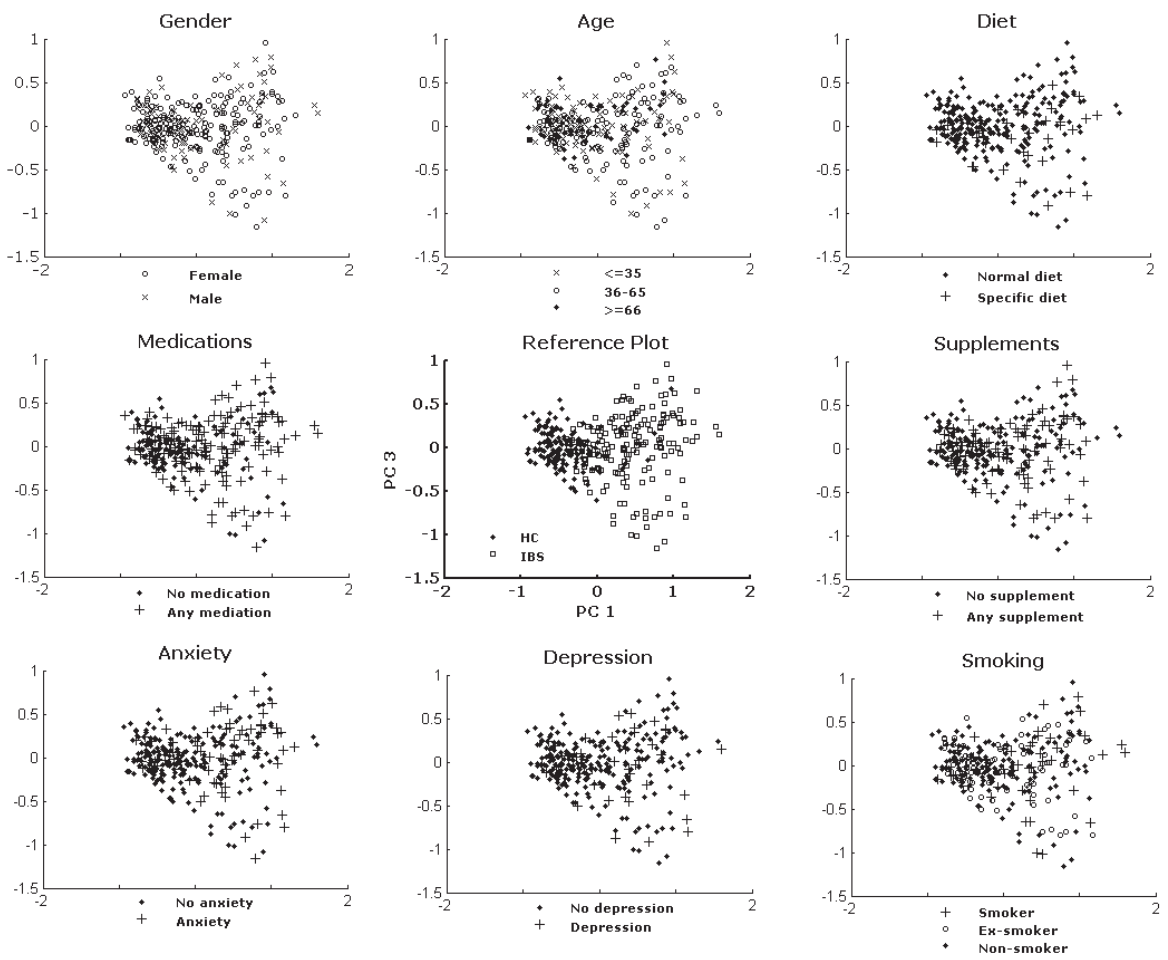

Figure S11.1 PCA score plots, defined by PC1 and PC3, on a proximity matrix obtained from the Random Forest model built on 16 selected VOCs, with the reference plot in the middle. Each point is a single chromatogram, with points shaped by external factors. For some factors (medication, supplement use and diet), the chromatograms were shape-coded if the subject was on any medication vs. no medication, on any supplement vs. no supplements, and when following a specific diet (vegetarian, gluten-free, etc.) vs. a normal diet. 


\section{Chapter 12}

General discussion 
Chapter 12 


\section{General discussion}

\section{Main findings}

In the thesis, we have focussed on pathophysiology, assessment of symptoms, and biomarkers in IBS. In Chapter 2, we demonstrated that small intestinal permeability is increased in IBS-D patients, but not in other subtypes. Alterations observed in gastroduodenal permeability in IBS and all subtypes were mainly contributable to confounders. Thereafter, in a randomized double blind placebo controlled clinical trial, we investigated potential beneficial effects of the oral administration of three Lactobacillus plantarum strains on small intestinal barrier function in healthy subjects, under stressed conditions (i.e. after intake of NSAIDs). Intake of L. plantarum TIFN101 resulted in most pronounced effects on gene transcription in duodenal biopsies, which were related to mucosal repair mechanisms (Chapter 3), and to maintenance of $\mathrm{T}$ and B-cell function and antigen presentation (Chapter 4).

Thereafter, we investigated differences between viscerally hyper- and normosensitive IBS patients (Chapter 5 and 6), and found that apart from age, gender, GI symptom severity and use of SSRIs, these two groups were comparable with regard to clinical characteristics and to a set of selected biomarkers.

Finally, the role of serotonin metabolism in IBS was investigated (Chapter 7). Both, systemic 5-HIAA and the 5-HIAA/5-HT ratio were lower in IBS patients compared to controls. Within the IBS group, differences were observed between IBS subgroups, being most pronounced in the IBS-M group.

In the second part of this thesis we focussed on symptoms in IBS. At first, we systematically reviewed the published literature and evaluated the currently available instruments for measurement of chronic abdominal pain in patients with IBS (Chapter 8). A wide variety of methods is used in IBS research, but many are known to have important limitations. In an exploratory study (Chapter 9), we then demonstrated that momentary symptom assessment may reduce or even exclude limitations of the methods that employ retrospective symptom assessment; for example, the exclusion of recall bias.

In the final two studies (Chapter 10 and 11), we identified two biomarker panels with high positive predictive values for IBS patients versus healthy controls, i.e. an 8-item biomarker panel of faecal and plasma markers and a 16-item biomarker panel based on the analysis of volatile organic compounds in exhaled breath. Furthermore, for both biomarker panels a good correlation was found to GI symptom severity in an IBS and a in a general population cohort. 


\section{Intestinal barrier in IBS and the interplay with live bacteria}

The multifactorial pathophysiology of IBS is represented by a complex interplay of a multitude of intestinal and central processes. We have investigated several of these processes known or presumed to be involved in the pathophysiology of IBS.

Dysregulation of complex processes of the gut barrier may lead to increased permeation of luminal factors into the mucosa or into the systemic circulation, and to local and systemic inflammation. The mechanism of increased permeation may either be an early event in the pathophysiology of IBS, or an epiphenomenon of the disorder, triggered by inflammation or dysregulation of the neuroendocrine system and brain-gut interaction. $^{1-3}$

Observational research is prone to confounding. Although a variety of factors, such as sex, ${ }^{4} \mathrm{BMI}^{5}$ use of alcohol or medication, ${ }^{6,7}$ are reported to affect gut barrier function, previous studies assessing intestinal permeability in IBS patients have not adjusted their results for potential confounders. In a group of 91 IBS patients and 94 healthy controls (Chapter 2), we found a significantly increased small intestinal permeability in IBS-D patients compared to controls. Furthermore, gastroduodenal permeability was significantly higher in the total IBS group and the IBS-D and IBS-C subtypes versus healthy subjects, but these differences were no longer significant after adjustment for confounders (i.e. mainly sex, BMI, and the use of prokinetics and laxatives). These new insights are relevant for the design of future studies assessing intestinal permeability in health and disease.

A complex interplay exists between the intestinal mucosa, the immune system and commensal bacteria. This interplay may be altered in IBS patients. Commensal intestinal bacteria prevent colonisation of pathogens, support the digestion of nutrients, co-regulate epithelial functions (e.g. differentiation, mucus production and defensin release), and affect the mucosal immune system, and tight junction protein synthesis. ${ }^{8}$ We administered indomethacin, a NSAID, as stress-model for the intestinal mucosa, to healthy subjects and investigated the effects of 7-day oral intake of three L. plantarum strains (all three commensals of the human GI tract) on small intestinal mucosa. In line with previous findings, ${ }^{9}$ indomethacin induced an increase in small intestinal permeability. This was not affected by any of the three bacterial strain interventions. However, one of the L. plantarum strains (i.e. TIFN101) induced strain dependent effects on gene transcription in intestinal mucosal cells: on gene modulation related to mucosal structure and on genes associated with maintenance of $\mathrm{T}$ and $\mathrm{B}$-cell function and antigen presentation. Furthermore, this $L$. plantarum strain enhanced memory T-cell responses against Staphylococcus aureus enterotoxin B superantigen and tetanus toxoid. Although we did not find a beneficial effect on intestinal permeability, the modulation of mucosal repair mechanisms after NSAID induced stress, and enhanced immune response against pathogens, may also contribute to improved overall barrier function. Furthermore, our findings demonstrate that even bacterial strains of the same species may have distinct different effects on the host, 
which points to careful selection of potential probiotic strains for specific target conditions or populations. In a recent meta-analysis, Ford et al. stated that probiotics have therapeutic potential in IBS. ${ }^{10}$ Despite rapidly increasing knowledge it remains to be established which individual bacterial species and strains are most beneficial for humans and for which disorder or disease. In the past decade, remarkable progress is made (mainly from animal and in vitro studies) with respect to understanding mechanisms by which consumed live bacteria, but also commensal microbiota, affect gut function. ${ }^{10-12}$ Nevertheless, this field of research is only in its early phase of development. Our studies have provided additional and new insights and leads for future studies with regard to the interactions between the intestinal barrier, immune system and bacteria present in the gut lumen.

\section{Heterogeneity of irritable bowel syndrome}

"Irritable bowel syndrome is a heterogeneous functional gastrointestinal disorder with a multifactorial aetiology that involves the interplay between both host and environmental factors".$^{13}$ This type of statement frequently is used as introduction to a research topic in scientific papers that deal with IBS. The heterogeneity of IBS has puzzled researches already for over a century, ${ }^{14}$ and is considered to limit treatment efficacy in IBS. Differences in aetiology are considered to underlie the diversity in clinical presentation in IBS. An alternative is that therapeutic actions should focus on more homogenous subgroups rather than on the general IBS patient population. Acknowledging this rationale, the Rome committee divided IBS in four subtypes based on predominant bowel habits. ${ }^{15}$ Although pathophysiological or intervention studies in IBS frequently focus on subtypes, usually an unselected IBS population is studied with insufficient power for separate subtype analyses. In addition, poor agreement (of $46 \%$ ) has been reported between IBS subtyping based on Rome II versus Rome III criteria, ${ }^{16}$ hindering a comparison of studies over time. Moreover, IBS symptoms and possibly also bowel habits fluctuate over time. In a prospective study about half of IBS-D and IBS-C patients were found to shift towards a different subtype, particularly to the mixed type, over a 12 -week time period. ${ }^{17}$ Therefore, the question rises how relevant these four IBS subtypes are for both research and clinical practice.

In Chapters 2, 5 and 7, we included sufficient numbers of IBS patients to investigate differences between IBS-D, IBS-C and IBS-M subtypes as a secondary objective. Studying intestinal permeability and serotonin metabolism, we found significant differences between IBS subtypes. Small intestinal permeability was increased only in the IBS-D group versus healthy controls (Chapter 2), while for IBS-C and IBS-M no differences in GI-permeability were observed. Furthermore, plasma 5-HIAA levels were decreased in all three subtypes of IBS when compared to healthy subjects. The 5-HIAA / $5-\mathrm{HT}$ ratio was decreased in the total IBS group and in the IBS-M subgroup (Chapter 7). Between the IBS subgroup significant differences were observed. The ratio 5-HIAA / 
5-HT was significantly lower in the IBS-M subgroup compared to the IBS-D group. This points to an association between altered serotonin metabolism and IBS-M subtype, possibly also a causative role for serotonin metabolism. The shift in predominant bowel habit that is characteristic for IBS-M patients is poorly understood. Up to now, serotonin metabolism has not been studied before in this subgroup of IBS patients. Since serotonin is known to affect gut motility and secretion, ${ }^{18}$ alterations in serotonin metabolism may contribute to the pathophysiology and clinical spectrum of IBS-M. However, more research is needed to further elucidate this association. Such research should include the investigation of changes of 5-HIAA and 5-HIAA / 5-HT ratio over time in IBS-M patients both in plasma and mucosal samples, and the relation in time to episodes with constipation and diarrhoea.

We did not observe significant differences between the IBS subtypes with regard to age, gender, BMI, lifestyle factors, psychological symptom severity, and mental and physical QoL-scores, which is in line with previous findings. ${ }^{19}$ Furthermore, GI symptom scores for abdominal pain or discomfort, bloating, flatulence, upper Gl symptoms, as well as overall intensity of GI complaints were comparable between the subtypes. The use of medication was equally distributed, apart from SSRIs, laxatives and anti-diarrheal drugs. Finally, we found a comparable prevalence of visceral hypersensitivity in all IBS subtypes (Chapter 5) and no major differences in the tested biomarkers panel (Chapter 11).

Despite an extensive characterisation, we were not able to clearly define the phenotype of three most prevalent IBS-subtypes ( $D, C$ and $M$ ) based on a variety of demographics, lifestyle, psychological and pathophysiological factors. These findings, together with possible changes in symptoms and subtypes over time, suggest that IBS patient clustering based on other factors is needed.

In this thesis, we investigated hyper- and normosensitive IBS patients. These groups differed with regard to age, gender, use of SSRIs, and GI symptom scores (Chapter 5). However, no differences were observed with regard to predominant bowel habits, psychological factors, quality of life, use medication other than SSRIs, time since IBS diagnosis, percentage of patients with post-infectious type IBS, or lifestyle factors. Furthermore, several biomarkers that represent specific pathophysiological mechanisms of IBS, such as faecal calprotectin, chromogranin A (CgA), human 8-defensin 2 (HBD2), short chain fatty acids (SCFAs), and plasma citrulline, 5-HT and 5-HIAA were not altered in the hypersensitive compared to the normosensitive IBS group. We conclude that subtyping IBS based on visceral perception does not lead to better sub-classification of IBS patients.

It should be noted that the fact that biomarker levels did not differ between hyper and normosensitive IBS patients, does not strictly exclude either low-grade-inflammation or altered neuroendocrine activity or intraluminal metabolic activity to be involved in the development of visceral hypersensitivity in IBS. Furthermore, we did not address all biological mechanisms associated with IBS. 
The current knowledge of IBS pathophysiology may not be sufficient to allow clustering of IBS patients based on differences in aetiology. The Maastricht IBS cohort formed the basis of several chapters of the current thesis. At this moment it consists of around 500 cases and 200 healthy controls, and inclusion is ongoing. The phenotype of the participants is well characterised, with regard to demographics, lifestyle, use of medication, comorbidity, QoL, psychological and GI symptoms, and an extensive dietary assessment. In subgroups of the cohort blood and faecal samples are collected. At present, further analyses are running on microbiota composition, metabolomics in exhaled air, host genetics and diet. These data, together with the data presented in this thesis, will be combined in a large database and will offer the chance for assumptionless cluster analyses, which may identify groups of patients with common characteristics and thereby may provide leads for further studies on underlying mechanisms contributing to symptom development and for therapeutic interventions.

\section{Symptom assessment in IBS patients: a paradigm shift?}

Chronic or recurrent abdominal pain, mostly of visceral origin, is a common symptom that is of great clinical significance in several medical areas, in particular for IBS. ${ }^{20}$ Reduction of this symptom burden usually is the primary aim of IBS treatments. ${ }^{15,21}$ However, the variety of retrospective questionnaires and provocation tests used in studies to measure abdominal pain in IBS (Chapter 8) makes the comparison between studies challenging. Many of these questionnaires have not been psychometrically tested and validated in the target population. Furthermore, these questionnaires are all based on autobiographical memory and are therefore biased, i.e. due to recall bias, as memory can be affected by different psychological and circumstantial factors. Comparing an end-of-day diary and an end-of-week questionnaire to a novel electronic momentary symptom assessment method, i.e. Experience Sampling Method (ESM), demonstrated that IBS patients report higher pain scores in retrospective questionnaires, compared to the average scores of momentary assessment (chapter 9). This finding was accompanied by a tendency of peak-pain reporting at the end of the day, which has been observed previously. ${ }^{22}$ The peak-reporting phenomenon was not observed for other GI symptoms, indicating that particularly abdominal pain is prone to recall bias. Interestingly, peak-pain reporting was not influenced by affective comorbidity in IBS patients. Electronic momentary symptom assessment in IBS may overcome limitations of the currently available methods, and can provide further insight in the fluctuation of symptom over the day. Intervention trials in patients are time-consuming, expensive and bare potential risks for participants. A potential advantage of the use of repeated measures in ESM and multi-level analytical methods is that it may increase the statistical power to detect differences over a period of time when compared to classical end-of-day diaries. This may result in smaller sample sizes in IBS intervention trials required to reach statistical significances. In this thesis the first step in the development and validation process of ESM for the use in IBS patients has 
been made. Further research is needed, and currently ongoing, to carefully select the questions to be used in ESM, to psychometrically test the sets of questions and to compare the scores to the current standardly used symptom assessment methods to this final set of questions in ESM, in a large group of IBS patients in normal conditions and during interventions.

\section{From symptoms to biomarker based diagnosis in IBS}

Underlying subtle and complex metabolic changes in IBS could in theory be detected by a combination of biomarkers with sufficient power to overcome hurdles of sensitivity in this heterogeneous patient population. Up to now, several studies have investigated single biomarkers and two studies explored a set of serum and genetic markers for $\mathrm{IBS}^{23-25}$ However, the predictive values of these markers are only low to moderate, which may be caused by an incomplete representation of the full range of mechanisms that are involved in the multifactorial pathophysiology of IBS.

In this thesis we focused, not only on IBS, but also on the measurement of gut health using biomarkers in a large general population cohort. Furthermore, a cohort of clinical IBS patients and age and gender matched healthy controls (without GI complaints) were included. Out of fifteen plasma and faecal markers a group of eight markers, i.e. IL-1 $\beta$, IL-6, IL12p70, TNF- $\alpha$, CgA, HBD2, calprotectin and caproate, was identified as the most discriminatory panel of biomarkers for the separation of IBS patients and healthy controls (sensitivity $88 \%$, specificity $87 \%$ ). This panel of markers correlated significantly to $\mathrm{Gl}$ symptom scores both in the IBS cohort $(r=0.6, p<0.001)$ and in the general population $(r=0.4, p<0.001)$. Furthermore, using a holistic metabolomics approach in the same cohorts a panel of 16 volatiles correctly predicted $89 \%$ (sensitivity) of IBS samples and $73 \%$ (specificity) of healthy controls, whit again good correlations to $\mathrm{GI}$ symptoms. These findings demonstrate that faecal and plasma biomarkers, which are indicative for different pathophysiological mechanisms of IBS, can be used as a highly predictive marker set for IBS patients, and even correlates to GI symptoms in subjects with more mild $\mathrm{Gl}$ symptoms in the general population. Furthermore, our findings acknowledge the value of measurement of VOCs in exhaled air of IBS patients and subjects in the general population for potential use as biomarkers.

Taking into account the fact that IBS is a functional GI disorder, and that the patient population is very heterogeneous, the sensitivity and specificity of both biomarkers panels can be considered as high. These studies are important steps towards a biomarker based diagnosis of IBS. However, further research is needed before the panels can be used in daily clinical practice. In particular, the value of the biomarker panels for the discrimination between IBS and other GI disorders, such as IBD or celiac disease, needs to be evaluated, as well as additional validation of the markers in other, independent, IBS cohorts.

The good correlation of the biomarker panels with Gl symptoms, suggests that both panels may be useful for the detection of changes in GI symptom severity, both in IBS 
patients and general population subjects, who have mild or moderate Gl complaints. Therefore, the panels could be useful as a parallel study outcome, next to symptom scores, of intervention trials aiming to reduce GI symptom burden in both target populations. Taking into account the high placebo response rates in IBS trials that use symptom scores as main outcome, ${ }^{26}$ a more objective biomarker panel that can measure responsiveness to therapies in these patients could be of great additional value. Here further research is needed to test the value of the biomarker panel with respect to responsiveness to therapies or interventions during longitudinal follow up.

\section{Putting irritable bowel syndrome in a broader perspective}

A large population based case-control study revealed high comorbidity rates for IBS and a range of gastrointestinal and extra-gastrointestinal diseases/disorders. Particularly an increased odds ratio (up to 6.7) was found for comorbid mental problems, including depression, anxiety disorders and sleeping problems, compared to control subjects. Moreover, a higher prevalence of pain related disorders were associated with IBS, such as fibromyalgia, headaches, chest pain, and low back pain. ${ }^{27}$ The discussion is still ongoing whether a subgroup of IBS patients show more generalized hypersensitivity instead of isolated visceral hypersensitivity. This would suggest the presence of more general pain disorders in these patients. Studies have assessed both visceral and somatic pain perception using artificial rectal and skin stimuli. Up to now, results are not uniform. In all studies increased visceral pain perception was found in IBS patients, but some reports indicate also somatic pain hypersensitivity in these subjects, ${ }^{28-31}$ while others found no differences in response to skin stimuli on the extremities between IBS patients and controls. ${ }^{32,33}$ A possible link between increased visceral and somatic pain perception in IBS patients, could be the involvement of central hyperalgesic mechanisms, possibly related to effects of stress. Stress is known to have both acute and chronic effects on gut function, including altered motility, barrier function and sensitivity. This occurs via alterations of the neural and (neuro)hormonal interaction between the gut, enteric nervous system, and the brain. ${ }^{34-36}$ Early life stress induced by maternal separation of rats has been found to lead to visceral hypersensitivity. Furthermore, these animal experiments suggest an essential role for mast cell degranulation and subsequent TRPV1 modulation in stress-related visceral hypersensitivity. ${ }^{37-39}$ Next to effects on the GI tract, stress may dysregulate processes in the central nervous system and among others the HPA-axis, resulting in systemic effects. This may provide the link between IBS and increased comorbidity of psychiatric and pain related disorders, and the presence of a more general hypersensitivity state. However, human studies that investigate the association between early life stress events and the development of IBS or visceral hypersensitivity in IBS patients, are currently lacking. ${ }^{40}$ 


\section{Integration of current knowledge into the daily clinical care of IBS patients}

In daily clinical practice dissatisfaction of IBS patients regarding the interaction with their physician is not uncommon, and may negatively impact IBS disease outcome. ${ }^{41}$ It is amongst others related to limited information and frustrations about the explanation of IBS by the physician, in particular with regard to aetiology. ${ }^{42-44}$ IBS patients worry more about having a serious disease than matched controls, which may partly be caused by a lack of understanding of their illness. ${ }^{27}$ As the effect of therapies in IBS is low to moderate, education of patients, which should lead to a better understanding of their symptoms and to development of adequate coping strategies is of outmost importance. A recent review by Hungin et al. provides an integrated model of IBS for clinicians to be used in daily clinical practice. It utilises the current knowledge regarding IBS aetiology, including altered peripheral regulation of gut function, altered brain-gut interaction, and psychological distress. ${ }^{45}$ The transfer of this knowledge via a model or scheme to IBS patients in an understandable, comprehensive way, may improve the outcome of IBS in affected subjects.

Since IBS is a heterogeneous disorder, is associated with triggers as stress or diet, and with frequent comorbidities (e.g. psychiatric disorders), a multidisciplinary approach may lead to better treatment outcomes compared to the standard care provided by GPs or gastroenterologists alone. This approach may involve disciplines such as behavioural psychology, psychiatry, physical therapy, dietetics and in case of specific comorbidities, disciplines as rheumatology, gynaecology, or urology. A onetime multidisciplinary education session was tested, which involved lectures to improve understanding of IBS and provided information about coping strategies by experts of different field to a class of up to six IBS patient. This approach led to a significant decrease in symptom burden in IBS patients, although placebo effect may have played a role, as randomization and blinding was difficult to achieve. ${ }^{46} \mathrm{~A}$ multidisciplinary approach leads to higher direct health care costs of the IBS treatment, but when successful, may reduce the economic burden on the long term. ${ }^{47}$ However, this approach remains to be tested and proven. Therefore, further research is needed to prove possible benefits on disease outcomes and the economic effects.

Avicenna (Ibn-Sīnā), the great Persian thinker and the writer of the Canon of Medicine (1025 AD), which was used for over 600 years as the guidance for standard medical practice in Europe and the Middle-East, stated that in medicine the absence of understanding does not warrant absence of existence. As described in this thesis, the current knowledge of IBS pathophysiology is far from complete. However, in contrast to only a few decades ago, our understanding of subtle organic changes that contribute to the development of this functional GI disorder has considerably expanded and is rapidly improving, which is likely to lead towards a more biomarker based diagnosis and considerably better treatment option for IBS in the (near) future. 


\section{References}

1. Sanchez de Medina F, Romero-Calvo I, Mascaraque C, Martinez-Augustin O. Intestinal inflammation and mucosal barrier function. Inflamm Bowel Dis. 2014;20:2394-404.

2. Keszthelyi D, Troost FJ, Jonkers DM, van Eijk HM, Lindsey PJ, Dekker J, et al. Serotonergic reinforcement of intestinal barrier function is impaired in irritable bowel syndrome. Aliment Pharmacol Ther. 2014; 40:392-402.

3. Vanuytsel T, van Wanrooy S, Vanheel H, Vanormelingen C, Verschueren S, Houben E, Salim Rasoel S, Tóth J, Holvoet L, Farré R, Van Oudenhove L, Boeckxstaens G, Verbeke K, Tack J. Psychological stress and corticotropin-releasing hormone increase intestinal permeability in humans by a mast celldependent mechanism. Gut. 2014;63:1293-9.

4. Marshall JK, Thabane M, Garg AX, Clark W, Meddings J, Collins SM; WEL Investigators. Intestinal permeability in patients with irritable bowel syndrome after a waterborne outbreak of acute gastroenteritis in Walkerton, Ontario. Aliment Pharmacol Ther. 2004;20:1317-22.

5. Teixeira TF, Souza NC, Chiarello PG, Franceschini SC, Bressan J, Ferreira CL, Peluzio Mdo C. Intestinal permeability parameters in obese patients are correlated with metabolic syndrome risk factors. Clin Nutr. 2012;31:735-40.

6. Kerckhoffs AP, Akkermans LM, de Smet MB, Besselink MG, Hietbrink F, Bartelink IH, Busschers WB, Samsom M, Renooij W. Intestinal permeability in irritable bowel syndrome patients: effects of NSAIDs. Dig Dis Sci. 2010;55:716-23.

7. Elamin EE, Masclee AA, Dekker J, Jonkers DM. Ethanol metabolism and its effects on the intestinal epithelial barrier. Nutr Rev. 2013;71:483-99.

8. Bischoff SC. 'Gut health': a new objective in medicine? BMC Med. 2011;9:24.

9. Bjarnason I, Williams P, Smethurst P, Peters TJ, Levi AJ. Effect of non-steroidal anti-inflammatory drugs and prostaglandins on the permeability of the human small intestine. Gut. 1986;27:1292-7.

10. Ford AC, Quigley EM, Lacy BE, Lembo AJ, Saito YA, Schiller LR, Soffer EE, Spiegel BM, Moayyedi P. Efficacy of prebiotics, probiotics, and synbiotics in irritable bowel syndrome and chronic idiopathic constipation: systematic review and meta-analysis. Am J Gastroenterol. 2014;109:1547-61.

11. Theodorou V, Ait Belgnaoui A, Agostini S, Eutamene H. Effect of commensals and probiotics on visceral sensitivity and pain in irritable bowel syndrome. Gut Microbes. 2014;5:430-6.

12. Gareau MG. Microbiota-gut-brain axis and cognitive function. Adv Exp Med Biol. 2014;817:357-71.

13. Rajilić-Stojanović M, Jonkers DM, Salonen A, Hanevik K, Raes J, Jalanka J, de Vos WM, Manichanh C, Golic N, Enck P, Philippou E, Iraqi FA, Clarke G, Spiller RC, Penders J. Intestinal Microbiota And Diet in IBS: Causes, Consequences, or Epiphenomena? Am J Gastroenterol. 2015;110:278-87.

14. Cumming W. Electro-galvanism in a peculiar affliction of the mucous membrane of the bowels. London Med Gazette. 1849;NS9:969-73.

15. Drossman DA, Corazziari, E., Delvaux, M., Spiller, R.C., Talley, N.J., Thompson, W.G., Whitehead, W.E. Rome III: The Functional Gastrointestinal Disorders, Third Edition: McLean, VA: Degnon Associates, Inc.; 2006.

16. Ersryd A, Posserud I, Abrahamsson H, Simren M. Subtyping the irritable bowel syndrome by predominant bowel habit: Rome II versus Rome III. Aliment Pharmacol Ther. 2007;26:953-61.

17. Mearin F, Baro E, Roset M, Badia X, Zarate N, Perez I. Clinical patterns over time in irritable bowel syndrome: symptom instability and severity variability. Am J Gastroenterol. 2004;99:113-21.

18. Keszthelyi D, Troost FJ, Masclee AA. Understanding the role of tryptophan and serotonin metabolism in gastrointestinal function. Neurogastroenterol Motil. 2009;21:1239-49.

19. Tillisch K, Labus JS, Naliboff BD, Bolus R, Shetzline M, Mayer EA, Chang L. Characterization of the alternating bowel habit subtype in patients with irritable bowel syndrome. Am J Gastroenterol. 2005;100:896-904.

20. Elsenbruch S. Abdominal pain in Irritable Bowel Syndrome: a review of putative psychological, neural and neuro-immune mechanisms. Brain Behav Immun. 2011;25:386-94.

21. U.S. Department of Health and Human Services FaDA, Center for Drug Evaluation and Research (CDER). Guidance for Industry Irritable Bowel Syndrome - Clinical Evaluation of Drugs for Treatment, 2012. http://wwwfdagov/downloads/Drugs/Guidances/UCM205269pdf. 
22. Weinland SR, Morris CB, Hu Y, Leserman J, Bangdiwala SI, Drossman DA. Characterization of episodes of irritable bowel syndrome using ecological momentary assessment. Am J Gastroenterol. 2011;106: 1813-20.

23. Corsetti M, Van Oudenhove L, Tack J. The quest for biomarkers in IBS-where should it lead us? Neurogastroenterol Motil. 2014;26:1669-76.

24. Jones MP, Chey WD, Singh S, Gong H, Shringarpure R, Hoe N, Chuang E, Talley NJ. A biomarker panel and psychological morbidity differentiates the irritable bowel syndrome from health and provides novel pathophysiological leads. Aliment Pharmacol Ther. 2014;39:426-37.

25. Lembo AJ, Neri B, Tolley J, Barken D, Carroll S, Pan H. Use of serum biomarkers in a diagnostic test for irritable bowel syndrome. Aliment Pharmacol Ther. 2009;29:834-42.

26. Ford AC, Moayyedi P. Meta-analysis: factors affecting placebo response rate in the irritable bowel syndrome. Aliment Pharmacol Ther. 2010;32:144-58..

27. Faresjo A, Grodzinsky E, Hallert C, Timpka T. Patients with irritable bowel syndrome are more burdened by co-morbidity and worry about serious diseases than healthy controls--eight years follow-up of IBS patients in primary care. BMC Public Health. 2013;13:832.

28. Stabell N, Stubhaug A, Flaegstad T, Nielsen CS. Increased pain sensitivity among adults reporting irritable bowel syndrome symptoms in a large population-based study. Pain. 2013;154:385-92.

29. Rossel P, Drewes AM, Petersen P, Nielsen J, Arendt-Nielsen L. Pain produced by electric stimulation of the rectum in patients with irritable bowel syndrome: further evidence of visceral hyperalgesia. Scand J Gastroenterol. 1999;34:1001-6.

30. Piche $M$, Arsenault $M$, Poitras $P$, Rainville $P$, Bouin $M$. Widespread hypersensitivity is related to altered pain inhibition processes in irritable bowel syndrome. Pain. 2010;148:49-58.

31. Verne GN, Price DD, Callam CS, Zhang B, Peck J, Zhou Q. Viscerosomatic facilitation in a subset of IBS patients, an effect mediated by N-methyl-D-aspartate receptors. J Pain . 2012;13:901-9.

32. Whitehead WE, Holtkotter B, Enck P, Hoelzl R, Holmes KD, Anthony J, et al. Tolerance for rectosigmoid distention in irritable bowel syndrome. Gastroenterology. 1990;98:1187-92.

33. Caldarella MP, Giamberardino MA, Sacco F, Affaitati G, Milano A, Lerza R, Balatsinou C, Laterza F, Pierdomenico SD, Cuccurullo F, Neri M. Sensitivity disturbances in patients with irritable bowel syndrome and fibromyalgia. Am J Gastroenterol. 2006;101:2782-9.

34. Konturek PC, Brzozowski T, Konturek SJ. Stress and the gut: pathophysiology, clinical consequences, diagnostic approach and treatment options. J Physiol Pharmacol. 2011;62:591-9.

35. Hyland NP, O'Mahony SM, O'Malley D, O'Mahony CM, Dinan TG, Cryan JF. Early-life stress selectively affects gastrointestinal but not behavioral responses in a genetic model of brain-gut axis dysfunction. Neurogastroenterol Motil. 2015;27:105-13.

36. Larauche M, Mulak A, Tache Y. Stress and visceral pain: from animal models to clinical therapies. Exp Neurol. 2012;233:49-67.

37. van den Wijngaard RM, Klooker TK, Welting O, Stanisor OI, Wouters MM, van der Coelen D, Bulmer DC, Peeters PJ, Aerssens J, de Hoogt R, Lee K, de Jonge WJ, Boeckxstaens GE. Essential role for TRPV1 in stress-induced (mast cell-dependent) colonic hypersensitivity in maternally separated rats. Neurogastroenterol Motil. 2009;21:1107-e94.

38. Stanisor OI, van Diest SA, Yu Z, Welting O, Bekkali N, Shi J, de Jonge WJ, Boeckxstaens GE, van den Wijngaard RM. Stress-induced visceral hypersensitivity in maternally separated rats can be reversed by peripherally restricted histamine-1-receptor antagonists. PloS One. 2013;8:e66884.

39. Winston J, Shenoy M, Medley D, Naniwadekar A, Pasricha PJ. The vanilloid receptor initiates and maintains colonic hypersensitivity induced by neonatal colon irritation in rats. Gastroenterology. 2007; 132:615-27.

40. Barreau F, Ferrier L, Fioramonti J, Bueno L. New insights in the etiology and pathophysiology of irritable bowel syndrome: contribution of neonatal stress models. Pediatric Res. 2007;62:240-5.

41. Dhaliwal SK, Hunt RH. Doctor-patient interaction for irritable bowel syndrome in primary care: a systematic perspective. Eur J Gastroenterol Hepatol. 2004;16:1161-6.

42. Bijkerk CJ, de Wit NJ, Stalman WA, Knottnerus JA, Hoes AW, Muris JW. Irritable bowel syndrome in primary care: the patients' and doctors' views on symptoms, etiology and management. Can J Gastroenterol. 2003;17:363-8. 
43. Stenner $\mathrm{PH}$, Dancey $\mathrm{CP}$, Watts $\mathrm{S}$. The understanding of their illness amongst people with irritable bowel syndrome: a Q methodological study. Soc Sci Med. 2000;51:439-52.

44. Casiday RE, Hungin AP, Cornford CS, de Wit NJ, Blell MT. Patients' explanatory models for irritable bowel syndrome: symptoms and treatment more important than explaining aetiology. Fam Pract. 2009; 26:40-7.

45. Hungin AP, Becher A, Cayley B, Heidelbaugh JJ, Muris JW, Rubin G, Seifert B, Russell A, De Wit NJ. Irritable bowel syndrome: an integrated explanatory model for clinical practice. Neurogastroenterol Motil. 2015 Feb 20.

46. Saito YA, Prather CM, Van Dyke CT, Fett S, Zinsmeister AR, Locke GR, 3rd. Effects of multidisciplinary education on outcomes in patients with irritable bowel syndrome. Clin Gastroenterol Hepatol. 2004;2: 576-84.

47. Canavan C, West J, Card T. Review article: the economic impact of the irritable bowel syndrome. Aliment Pharmacol Ther. 2014;40:1023-34. 
Summary 


\section{Summary}

Irritable Bowel Syndrome is a highly prevalent functional gastrointestinal disorder, which often results in high symptom burden and significant impairment in quality of life of affected subjects. The multifactorial aetiology of IBS will contribute to various symptoms and distinct phenotypes. This thesis contained three parts, that focussed on i) the pathophysiology of IBS, ii) symptom assessment in IBS patients and iii) the development of new biomarker panels for IBS.

The aetiology of IBS is multifactorial, complex and incompletely understood. In part one of this thesis, we investigated several potential pathophysiological mechanisms of IBS, and modulation thereof.

In chapter 2, we studied alterations in intestinal permeability in IBS patients, with particular emphasis on IBS subtypes. A validated multi-sugar test was used to investigate gastro-duodenal, small intestinal, colonic and whole gut permeability in a large group of IBS patients and age and gender matched healthy controls. All analyses were adjusted for possible confounding effects of demographics, lifestyle factors, the presence of psychological symptoms and the use of medication. We found that small intestinal permeability, indicated by elevated urinary excretion of orally ingested lactulose and rhamnose ratio, was increased in diarrhoea predominant IBS patients when compared to healthy controls. This effect was still present after adjustment for possible confounders. Gastro-duodenal permeability, indicated by urinary sucrose excretion, was increased in the total IBS group, as well as in the diarrhoea and constipation predominant subtype. However, after adjustment for possible confounding effects, this association was no longer significant. For colonic and whole gut permeability, no significant differences between groups were found.

The findings presented in this study point to a different pathophysiological mechanism in the IBS-D subtype when compared to the other IBS subtypes, and demonstrate that adjustment for possible confounding factors is essential when investigating intestinal permeability, especially in a heterogeneous disorder as IBS.

Life bacteria, present in the gut as microbiota or ingested orally, interact with the host and may affect gut and immune function of humans. It is postulated that this interaction may be altered in IBS patients. In chapter $\mathbf{3}$ and chapter $\mathbf{4}$ of this thesis we investigated the effects of oral administration of three different Lactobacillus plantarum strains (all commensals of the human Gl tract) on the intestinal barrier and mucosal and systemic immune function. These studies were carried out in healthy human subjects, after consumption of the NSAID indomethacin. NSAIDs are frequently consumed drugs, also by patients with IBS. These drugs have immuno-modulatory and enteropathic effects. In our studies, NSAIDs have been used as stress-model for the intestinal mucosa. Indomethacin induced an increase in small intestinal permeability, 
which was in line with previous findings, but which was not affected by any of the bacterial interventions. However, intake of the bacterial strains did induce straindependent effects on gene transcription in intestinal mucosal cells. L. plantarum TIFN101 showed the most pronounced modulatory properties with regard to mucosal gene transcription, with positive effects on gene transcription of repair processes in the compromised intestine of healthy subjects, and upregulated genes associated with maintenance of $\mathrm{T}$ and $\mathrm{B}$-cell function and antigen presentation. Furthermore, it enhanced the memory T-cell responses against Staphylococcus aureus enterotoxin B superantigen and tetanus toxoid. These studies provided further mechanistic insight how three different $L$. plantarum strains affect the host, and demonstrated that effects are strain specific. Furthermore, our findings provide new insights for the interactions between intestinal barrier, immune system and bacteria present in the gut lumen. This interplay is presumed to play an important role in the pathophysiology of IBS.

In chapter 5 and chapter $\mathbf{6}$, we focused on visceral hypersensitivity in IBS patients, with emphasis on differences in clinical characteristics and specific biomarkers in hyperversus normosensitive IBS patients. Visceral sensitivity was assessed using an electronic barostat with rectal balloon distention. Interestingly, the hypersensitive IBS patients were comparable to those patients classified as normosensitive with regard to demographic and lifestyle characteristics and medication use. Only younger age, female sex and the use of SSRI medication was significantly associated with the presence of visceral hypersensitivity in IBS patients. However in a multivariate logistic regression analysis only higher age was found to be significantly associated with lower odds for having hypersensitivity. No differences were observed with regard to predominant bowel habits, psychological factors, quality of life, medication use other than SSRIs, time since IBS diagnosis, percentage of patients with post-infectious type IBS, or lifestyle factors. Furthermore, the concentrations of calprotectin, human B-defensin 2, chromogranin A, and short chain fatty acids (measured in faeces), and serotonin, its principal metabolite 5-hydroxyindoleacetic acid (5-HIAA) and citrulline (measured in plasma), reflecting several potential underlying mechanisms, did not differ significantly between normosensitive and hypersensitive IBS patients.

Taken together, our data raise the question whether the assessment of visceral sensitivity in IBS patients has relevant implications in daily clinical practice, since the differences between hyper- and normosensitive IBS patients were moderate. Nevertheless, it has to be acknowledged that the use of intestinal provocation tests in scientific research in this target population in the past decades has provided unique knowledge with regard to neural pain processing of intestinal stimuli and may also provide keys to tailored therapeutic interventions.

In chapter 7 we have investigated platelet poor plasma serotonin (5-HT) and 5-HIAA levels and their ratio in a large cohort of IBS patients and healthy controls, including 
extensive IBS subgroup analyses. While no differences were detected in plasma 5-HT between groups, 5-HIAA concentrations were significantly lower in IBS patients compared to controls, which was also true for 5-HIAA/serotonin ratio. Furthermore, subtype analysis (after adjustment for possible confounding factors) demonstrated that both 5-HIAA and 5-HIAA/serotonin ratio were significantly decreased, particularly in IBS patients with a mixed stool pattern (i.e. IBS-M). Furthermore, our data point to an influence of gender on the serotonin metabolism in IBS, but not of Gl symptoms, anxiety and/or depression, lifestyle factors, or medication use.

This study demonstrated that although fasting serotonin plasma levels were not altered in IBS patients compared to controls, the differences in 5-HIAA levels may reflect altered serotonin metabolism in IBS patients, in particular in the IBS-M subtype.

Consensus on standard methods to assess chronic abdominal pain in patients with IBS is currently lacking. In the second part of this thesis we focused on methods for GI symptom assessment in IBS patients. First, in chapter 8 the currently available instruments for assessing chronic abdominal pain in IBS patients have been evaluated in a systematic review. There was large variety in assessment tools used in the included studies, which hindered comparison between studies and pooling of data for a metaanalyses. Provocation tests, such as the rectal barostat, are often applied in studies that include IBS patients, and are useful tools to assess an acute response to artificial intestinal stimuli. However, the outcomes do not correlate well to chronic abdominal pain severity as assessed by retrospective questionnaires. Retrospective symptom assessment by paper questionnaires or diaries carry the risk of recall and ecological bias. Nevertheless, at this moment retrospective Gl-symptom severity scales are frequently used, are easy to apply, and are the best validated methods for symptom assessment in IBS patients. However, these questionnaires in general include only the assessment of abdominal pain intensity. There is need for validated instruments to assess chronic abdominal pain in IBS patients that overcome the limitations of the currently available methods. Momentary symptom assessment, may be an alternative that can eliminate recall bias when measuring abdominal pain, but is a relatively new method in IBS studies, and further validation is needed.

In line with the conclusion of this review, we conducted a pilot study (chapter 9) to investigate the potential of Experience Sampling Method (ESM) for symptom assessment in IBS patients. ESM is based on digital momentary symptom assessment, which eliminates recall bias, reduces ecological bias and provides repeated symptom measures during the day. We found that end-of-day diary abdominal pain scores were higher compared to corresponding ESM mean-scores in IBS patients. The difference was even more pronounced for the end-of-week GSRS scores compared to ESM meanscores. These findings indicate that subjects report peak pain rather than average pain scores in retrospective questionnaires. For flatulence, comparable results were found. Nausea and belching scores showed small, but significant differences between end-of- 
day diary and ESM. All tested symptoms did score higher on GSRS compared to ESM mean-scores. Affective comorbidity did not influence differences in pain reporting between methods. This study showed that ESM can provide more insight in symptom course and potential triggers, and thereby may lead to a better understanding of IBS symptomatology.

The diagnosis of IBS is symptom-based and validated reliable biomarkers could aid the diagnostic process in daily clinical practice and scientific research. In the third and final part of this thesis, we focused on the development and validation of new biomarkers for IBS and their potential implication in the general population. Although considered a functional GI disorder, accumulating evidence exists for various subtle organic changes in IBS patients compared to healthy controls. These associations offer the chance for the development of biomarkers for IBS. The predictive values of several single markers investigated previously are however found to be low to moderate. We hypothesized that a combination of biomarkers that relate to several pathophysiological mechanisms of IBS is assumed to have sufficient power to overcome hurdles of sensitivity in this heterogeneous patient population with complex underlying aetiology and rather subtle organic changes. The predictive values of the markers investigated previously are low to moderate.

In chapter 10 we combined faecal and plasma biomarkers related to several domains of gut health. All markers were measured in two large cohorts, i.e. a clinical IBS cohort with age and gender matched healthy controls without Gl symptoms, and a general population cohort from which Gl-symptom scores were available. An 8-item biomarker panel consisting of IL-1 $\beta$, IL-6, IL12p70, TNF- $\alpha$, CgA, HBD2, calprotectin and caproate, was identified to discriminate IBS patients form healthy controls with high sensitivity of $88 \%$ and specificity of $87 \%$. When using the same 8 -item biomarker panel to discriminate IBS subtypes from controls, comparable results were found. Furthermore, the biomarker panel correlated well with Gl-symptom scores in a general population cohort.

In chapter 11, a holistic approach was used to identify new biomarkers, i.e. volatile organic compounds in exhaled air. A set of 16 VOCs that best distinguished IBS patients from healthy controls was identified. The VOC-biomarker set correlated significantly with Gl symptoms in two independent cohorts. The set correctly predicted $89 \%$ of the IBS patients and $73 \%$ of the healthy controls.

Although further research is needed to test the applicability of these biomarker panels in daily clinical practice, these findings are important steps towards biomarkers based diagnosis of IBS. 
In chapter 12, we give an overview of the major findings of this thesis, tried to integrate the different concepts that were discussed in the different chapters, and provide new hypothesis to be investigated in future studies that focus on IBS. 
Samenvatting 


\section{Samenvatting}

Het Prikkelbare Darm Syndroom (PDS), oftewel Irritable Bowel Syndrome (IBS) in het Engels, is een veel voorkomende functionele maagdarmaandoening. Bij PDS-patiënten leiden de klachten die gerelateerd zijn aan deze aandoening tot een duidelijke vermindering van de kwaliteit van leven. De multifactoriële etiologie van PDS levert een verscheidenheid aan symptomen en fenotypen op.

Dit proefschrift is opgedeeld in drie onderdelen, die zich richtten op i) de pathofysiologie van PDS, ii) het meten van symptomen bij PDS-patiënten en iii) de ontwikkeling en validatie van nieuwe biomarker sets voor PDS.

De etiologie van PDS is multifactorieel, complex en wordt nog steeds onvoldoende begrepen. In deel één van dit proefschrift bestudeerden wij diverse potentiële pathofysiologische mechanismen van PDS.

In hoofdstuk 2 richtten wij ons op de veranderingen in intestinale permeabiliteit in PDS-patiënten ten opzichte van gezonde controlepersonen. Daarnaast werd specifiek aandacht besteed aan de verschillen binnen PDS-subtypen, te weten het constipatie(IBS-C) en diarree-predominante subtype (IBS-D) en het subtype met een gemengd defecatiepatroon (IBS-M). Een gevalideerde multi-suiker test werd gebruikt om de gastroduodenale, dunne darm-, colon- en de volledige maagdarm-permeabiliteit te onderzoeken in een grote groep PDS-patiënten en in op leeftijd- en geslacht-gematchte gezonde controles. Alle analyses werden gecorrigeerd voor potentiële confounders, zoals demografische en leefstijlfactoren, psychologische symptomen en het gebruik van medicijnen. In deze studie vonden wij dat de dunne darmpermeabiliteit verhoogd was in PDS-patiënten met het diarree-predominante subtype in vergelijking tot gezonde controles. Dit verschil was ook aanwezig na correctie voor mogelijke confounders. De gastro-duodenale permeabiliteit was verhoogd in de totale PDS-populatie en in de diarree- en constipatie-predominante subtypen. Echter, na correctie voor confounders waren deze verschillen niet meer statistisch significant. Voor colon- en volledige maagdarm-permeabiliteit werden geen verschillen gevonden tussen de diverse groepen.

De bevindingen, die we in deze studie gepresenteerd hebben, wijzen op een ander pathofysiologisch mechanisme in PDS-patiënten met het diarree-predominante subtype ten opzichte van de andere PDS subtypen. Tevens laat deze studie zien dat bij het bestuderen van intestinale permeabiliteit, in het bijzonder in een heterogene populatie als de PDS-patiënten, het corrigeren voor mogelijke confounders van groot belang is.

Diverse studies tonen aan dat de samenstelling van de darmflora, ofwel microbiota, anders is bij patiënten met PDS en mogelijk een rol speelt bij het ontstaan van klachten. De aanwezige bacteriën in de darm, maar ook oraal ingenomen bacteriën gaan 
interacties aan met de gastheer en hebben o.a. invloed op de functie van het maagdarmkanaal en het immuunsysteem. In hoofdstuk 3 en hoofdstuk 4 van dit proefschrift hebben wij de effecten van drie oraal ingenomen Lactobacillus plantarum stammen op de intestinale barrièrefunctie en het mucosale en systemische immuunsysteem onderzocht. De drie stammen zijn van oorsprong commensale bacteriën van het maagdarmkanaal. De twee studies werden uitgevoerd in tien gezonde proefpersonen die gedurende testperiodes van zeven dagen elke dag één van de drie stammen innamen. Tevens kregen zij de NSAID indometacine toegediend op voor de testperiodes en op laatste dag van de periodes. NSAID's, die een pijnstillende en ontstekingsremmende werking hebben, worden frequent gebruikt, onder andere door PDS-patiënten. Deze middelen hebben ook een immuunmodulerend en enteropathisch effect. In onze studies werden de NSAID's gebruikt als een stressmodel voor de intestinale mucosa van de gezonde proefpersonen. Zoals ook in eerdere studies aangetoond, leidde de inname van indometacine tot een verhoogde dunne darmpermeabiliteit. Dit effect kon niet tenietgedaan worden door de inname van de $L$. plantarum stammen. Desalniettemin liet de inname van de bacteriële stammen wel een stamafhankelijk effect zien op de gentranscriptie van de intestinale mucosale cellen: de L. plantarum TIFN101 bacteriën hadden de meest uitgesproken modulerende eigenschappen. De veranderingen hadden betrekking op de gentranscriptie van reparatieprocessen in het door de NSAID's gecompromitteerd darmweefsel en de opregulatie van genen die geassocieerd zijn met het behoud van de T- en B-celfunctie en antigeenpresentatie. Tevens versterkten deze bacteriën de respons van $T$ geheugencellen tegen het Staphylococcus aureus enterotoxin B superantigeen en het tetanus toxine.

In deze twee studies presenteerden wij nieuwe mechanistische inzichten met betrekking tot de interactie van de oraal ingenomen $L$. plantarum stammen met de mens, zoals de dambarrière en de intestinale en systemische immuunactivatie. Van beide mechanismen wordt tevens gedacht dat deze een rol spelen in de pathofysiologie van PDS.

In hoofdstuk 5 en hoofdstuk 6 hebben we ons gericht op viscerale hypersensitiviteit, en met name op de verschillen tussen normo- en hypersensitieve PDS-patiënten. Hierbij hebben we zowel gekeken naar klinische kenmerken van deze groepen als verschillen in biologische markers. Viscerale sensitiviteit werd gemeten met behulp van een elektronische barostat, gebruik makend van een rectale opblaasbare ballon. Uit onze studies bleek dat de hypersensitieve PDS-patiënten op veel gebieden vergelijkbaar zijn met de normosensitive groep. Hypersensitieve PDS-patiënten waren wel jonger, vaker vrouw en gebruikten vaker SSRI-medicatie. Echter, een multivariate logistische regressie analyse laat zien dat alleen de jongere leeftijd geassocieerd is met de viscerale hypersensitiviteit bij PDS-patiënten. Er werden geen verschillen met betrekking tot maag-darmklachten, psychologische factoren, kwaliteit van leven, het gebruik van 
medicatie behoudens SSRIs, de duur van de klachten (gemeten als tijd sinds diagnose), en leefstijlfactoren. Daarnaast bleken ook de concentraties van verschillende markers, die gerelateerd zijn aan mogelijke onderliggende pathofysiologische mechanismen van PDS, niet te verschillen tussen de normo- en hypersensitieve PDS-patiënten. Deze biomarkers waren calprotectine, humaan B-defensine 2, chromogranine $A$ en korte keten vetzuren gemeten in feces, en plasma citrulline, serotonine en zijn metaboliet 5-hydroxyindoleacetic zuur (5-HIAA).

Samenvattend kan gesteld worden dat de resultaten van deze twee studies tonen dat de demografische en klinische verschillen net als de verschillen in de gemeten biomarker concentraties zeer beperkt zijn tussen normo- en hypersensitieve patiënten. Desalniettemin dient benadrukt te worden dat het gebruik van intestinale provocatietesten in experimentele studies met deze patiëntenpopulatie in de afgelopen decennia tot unieke kennis heeft geleid wat betreft de mechanismen hoe viscerale pijn wordt geregistreerd en wordt ervaren.

In hoofdstuk 7 hebben we serotonine (5-HT) en 5-HIAA concentraties gemeten in trombocyten-arm plasma en de ratio van de twee markers onderzocht in PDS-patiënten en gezonde controles. In deze studie werd ook specifiek aandacht besteed aan de PDSsubtypen. Er werden geen verschillen gevonden in 5-HT concentraties tussen de groepen, maar 5-HIAA-waarden bleken significant lager te zijn in PDS-patiënten in vergelijking tot gezonde controles. Eenzelfde resultaat werd gevonden voor de 5$\mathrm{HIAA} / 5-\mathrm{HT}$ ratio. Beide bevindingen bleven statistisch significant na correctie voor potentiele confounders. Tevens ontdekten we dat de 5-HIAA concentratie en de 5HIAA/5-HT ratio met name verlaagd waren in PDS-patiënten met het subtype gekenmerkt door een gemengd defecatiepatroon (IBS-M). Geslacht bleek als enige van de onderzochte parameters invloed te hebben op de waarden van de geteste serotoninemarkers in PDS-patiënten, terwijl geen effect werd gezien van maagdarmklachten, angst en depressieve symptomen, leefstijlfactoren of het gebruik van medicatie.

In deze studie hebben wij laten zien dat weliswaar de serotoninewaarden in plasma van PDS-patiënten, in bloed afgenomen bij alle proefpersonen in nuchtere toestand, niet verschillen ten opzichte van gezonde controles, maar dat er wel verschillen zijn in 5-HIAA en 5-HIAA/5-HT ratio tussen PDS-patiënten en gezonde proefpersonen en dat dit met name kenmerkend was voor het IBS-M subtype. Dit kan erop wijzen dat verstoringen in het serotoninemetabolisme een pathofysiologisch rol spelen in de betreffende patiëntenpopulatie.

PDS is een op symptomen gebaseerde diagnose en symptoomscores worden gebruikt voor follow-up en het kwantificeren van therapie-effecten. Samen met een veranderd defecatiepatroon is buikpijn een belangrijk kenmerk van PDS. Op dit moment bestaat er geen consensus over de beste methode voor het meten van buikpijn bij PDS-patiënten. 
In het tweede deel van dit proefschrift richten wij ons op verschillende methodes voor het meten van buikpijn en andere gastrointestinale symptomen. Allereerst evalueerden en vergeleken we in hoofdstuk $\mathbf{8}$ in een systematische review, de verschillende methodes die beschikbaar zijn voor het meten van buikpijn bij PDS-patiënten. We hebben geconcludeerd dat er een grote verscheidenheid aan instrumenten wordt gebruikt en dat dit de mogelijkheid beperkt om studieresultaten met elkaar te vergelijken in meta-analyses. Provocatietesten, zoals de rectale barostat, worden frequent gebruikt in studies waarin PDS-patiënten geïncludeerd worden en zijn nuttige instrumenten voor het kwantificeren van een acute respons op een kunstmatig opgewekte intestinale stimulus. De uitkomst van deze methoden correleren echter slecht met de scores van chronische buikpijn in retrospectieve vragenlijsten. Retrospectieve symptoommeting met behulp van papier of elektronische vragenlijsten of dagboeken dragen het risico met zich mee van zogeheten recall- en ecologische bias. Desalniettemin worden de algemene PDS-vragenlijsten, met name de papieren versies, nog zeer frequent gebruikt in studies en zijn ze eenvoudig voor zowel de patiënten als de onderzoekers en zijn ze de best gevalideerde methoden voor het meten van gastrointestinale symptomen in deze patiëntenpopulatie. Een nadeel is dat deze vragenlijsten zich meestal alleen richten op de intensiteit van de pijn, en geen rekening houden met fluctuaties over de tijd of factoren die pijn beïnvloeden. Een nieuwe methode in dit vakgebied, de 'Experience Sampling Method' (ESM), kan een alternatief zijn en kan de recall- en ecologische bias van retrospectieve vragenlijsten reduceren. Tevens biedt het meer statistische power om verschillen te meten door de repetitieve en momentane symptoommeting die de kern vormt van deze meetmethode. In hoofdstuk 9 presenteerden wij de resultaten van een pilotstudie waarin we een standaard papieren symptoomdagboek (ingevuld aan het einde van iedere dag), een vragenlijst die ingevuld werd aan het einde van een week (de GSRS) en ESM-scores (ingevuld zes tot tien maal per dag gedurende een week) met elkaar vergeleken hebben. We vonden dat buikpijnscores van de dagboeken hoger waren dan de gemiddelde scores van gemeten met ESM gedurende dezelfde periode. Deze verschillen waren nog meer uitgesproken toen we de ESM-scores vergeleken met de GSRS-scores. De resultaten toonden dat PDS-patiënten geneigd zijn de maximale pijn van de dag of de week onthouden en deze rapporteren in retrospectieve vragenlijsten. Voor flatulentie, misselijkheid en opboeren werden ook hogere scores gevonden in de dagboeken in vergelijking tot ESM, maar deze waren minder uitgesproken in vergelijking tot buikpijn. In deze studie hebben we ook laten zien dat ESM meer inzicht geeft in het verloop van gastrointestinale klachten over de dag bij PDS-patiënten. Deze methode biedt ook de mogelijkheid om potentiele uitlokkende factoren van bijvoorbeeld buikpijn te identificeren en kan hierdoor leiden tot een beter begrip van PDS-symptomatologie.

Daar symptoomscores door diverse (subjectieve) factoren kunnen worden beïnvloed, zouden betrouwbare biomarkers voor PDS een belangrijke bijdrage kunnen leveren in 
zowel het diagnostisch als follow-up proces in deze patiëntenpopulatie. In het derde en laatste deel van dit proefschrift richtten wij ons op het identificeren en valideren van nieuwe biomarker sets voor PDS, correlatie met symptoomscores en de mogelijke implicatie van een dergelijke biomarker set in de algemene populatie. Ondanks het feit dat PDS een functionele maagdarmaandoening is, bestaat er steeds meer bewijs voor een reeks van subtiele verschillen in diverse pathofysiologische factoren tussen PDSpatiënten en gezonde controles. Deze associaties bieden de mogelijkheid om multidomein biomarker sets te ontwikkelen die PDS-patiënten kunnen identificeren met een betere specificiteit en sensitiviteit dan de op dit moment bestaande solitaire biomarkers. Onze hypothese was dan ook dat een biomarkerset alleen dan goede diagnostische waarden zal hebben als deze de multifactoriële en complexe etiologie van PDS representeert. In hoofdstuk $\mathbf{1 0}$ hebben we daarom een reeks fecale en bloedplasma-biomarkers gemeten die allen een relatie hebben tot een bepaald domein van darmgezondheid geassocieerd met PDS. Hiervoor hebben we gebruik gemaakt van twee grote cohorten, namelijk een klinische PDS-cohort met leeftijd- en geslachtgematchte gezonde controles zonder maagdarmklachten, alsook een algemene populatie-cohort (Lifelines DEEP). Dit heeft geleid tot de identificatie van een biomarker set van 8 markers, bestaande uit IL-1 $\beta$, IL-6, IL12p70, TNF- $\alpha$, chromogranine A, humaan $\beta$-defensine 2 , calprotectine en het korte keten vetzuur caproaat. Deze biomarker set had een sensitiviteit van $88 \%$ en specificiteit van $87 \%$ voor de discriminatie van PDSpatiënten versus gezonde controles. Vergelijkbare resultaten werden gevonden voor alle PDS-subtypen. Daarnaast bleek de biomarker set goed te correleren met verschillende symptoomscores in zowel het PDS-cohort alsook het algemene populatiecohort.

In hoofdstuk 11 hebben we gekozen voor een holistische benadering. We hebben alle vluchtige organische stoffen (VOC's) gemeten in uitademingslucht van proefpersonen uit de bovengenoemde twee cohorten. Een set van $16 \mathrm{VOC}$ 's bleek het beste onderscheid te kunnen maken tussen PDS-patiënten en de gezonde controles met een specificiteit van $89 \%$ en sensitiviteit van $73 \%$. Ook in deze studie correleerde deze nieuwe biomarker-set goed met de ernst van gastrointestinale symptomen binnen de twee cohorten.

Uiteraard is verder onderzoek en validatie nodig, maar de huidige bevindingen van deze twee studies zijn belangrijke stappen in de richting van een op biomarkers gebaseerde diagnose en follow-up van PDS.

Tot slot gaven wij in hoofdstuk 12 een overzicht van de belangrijkste bevindingen in dit proefschrift, werden de verschillende concepten geïntegreerd en voortbordurend op de resultaten in dit proefschrift, presenteerden we nieuwe hypotheses voor vervolgonderzoek op het gebied van PDS. 
Valorisation addendum 


\section{Valorisation addendum}

With this thesis we aim to contribute to the true goal of medical research, that is to improve our understanding of human physiology and pathology, but also to find new approaches to improve and optimise healthcare. This thesis focused on a highly prevalent functional gastrointestinal (GI) disorder, i.e. Irritable Bowel Syndrome (IBS). IBS is associated with significant impact on patients' quality of life as well as on societal and health care costs. The studies we presented will add to further insight in the pathophysiology of IBS and provide tools to improve diagnosis and patient monitoring.

IBS is the most commonly diagnosed GI condition, ${ }^{1}$ characterized by abdominal pain or discomfort and altered bowel habits. Other GI symptoms such as bloating, flatulence, nausea and belching, are also frequently reported by patients. ${ }^{2}$ Although the prevalence of IBS varies geographically, a worldwide estimate of the prevalence is $11 \%$, which is comparable to findings in the Dutch population. ${ }^{3}$ Furthermore, IBS is more common in women than in man, with a 2:1 ratio. The exact reasons for this female predominance are not yet clear. ${ }^{4}$

Predominant symptoms and possible triggers vary between patients. Furthermore, in most patients, IBS is a chronic relapsing disorder in which symptoms may vary during the day as well as over time. ${ }^{5}$ Symptom burden in IBS patients can be considerable, contributing to significant impairment of quality of life (QoL). ${ }^{6}$ This is found not only when compared to the general population, but also in comparison with patients with other medical conditions, such as diabetes mellitus and even end-stage renal disease. ${ }^{7}$ The high symptom burden and impact on QoL has a negative impact on work productivity. Therefore IBS is not only associated with significant healthcare costs but also with overall costs to society. The estimated annual healthcare costs per newly diagnosed IBS patient, for example, is up to 7.500 US dollar in the USA and ranges between 500 and 1.000 US dollar in Europe. Cost to industry in Europe through absenteeism and presenteeism related to IBS is estimated between 500 and 1000 US dollar per patient annually. ${ }^{8}$

Solving the riddle of IBS starts with understanding the mechanisms from which symptoms originate. The aetiology of IBS is complex, multifactorial and incompletely understood. Several possible aetiological factors are found to be associated with IBS, including intestinal (e.g. impaired intestinal barrier and altered microbiota composition and activity) and brain-derived factors (for example anxiety and depression) as well as the interaction between the brain and the gut. ${ }^{9}$ Despite decades of scientific research and rapidly improving knowledge in the field of neurogastroenterology and motility, there is still much unclear with regard to the pathophysiology, the diagnostic process, standard therapeutics and prognosis of IBS or subgroups thereof. ${ }^{10}$ The IBS patient population is very heterogeneous. It is a syndrome based on a cluster of symptoms and 
generally divided in 4 subgroups based on predominant bowel habits (i.e. diarrhoea, constipating, a mix of both, or undefined). Therefore, it can be assumed that the main pathophysiological mechanisms may differ between IBS patients and the efficacy of therapies that target these mechanisms may differ per patient group. It is therefore of utmost importance to gain more insight in the underlying pathophysiological mechanism of IBS and to cluster patients in relevant subgroups. In the current thesis we investigated several potential pathophysiological mechanisms of IBS and focused on differences between groups of IBS patients.

As described in this thesis, the intestinal barrier function was found to be impaired resulting in increased intestinal permeability. However, this was influenced by a large number of host factors, such as use of medication. After adjustment for among others medication use the intestinal permeability was only found to be increased in the small bowel of the diarrhoea-predominant subtype (IBS-D). On one hand, these findings show that it is very important to take variations in host factors into account when performing mechanistic studies. On the other hand, this indicates that intestinal barrier function may contribute to symptom generation by increased permeation of toxic substances that may affect for example neuroendocrine and immune function, in IBS-D patients but not in other subtypes. A variety of agents have been demonstrated to improve and restore disturbed barrier function in experimental studies. Recently, faecal microbiota transplantation has been shown to re-establish mucosal barrier function in animal models, ${ }^{11}$ pointing to a role of the intestinal microbiota in maintenance of stable barrier function. Furthermore, previous studies showed that the administration of living bacteria into the gut lumen of healthy subjects affected the rearrangement of tight junction proteins. ${ }^{12}$ In line with these findings, we have demonstrated that potentially beneficial bacteria, i.e. different $L$. plantarum strains, may affect gene transcription related to intestinal barrier function as well as the mucosal and systemic immune system in healthy subjects in whom barrier function was disturbed by intake of nonsteroidal anti-inflammatory drugs (NSAIDs). However, whether such interventions have potential clinical implication for IBS patients, especially in patients with IBS-D, still has to be investigated.

Furthermore, in this thesis we focused on other potentially affected biological processes in IBS patients such as the serotonin metabolism and visceral perception. Both are potential targets in future therapeutic interventions. The serotonin metabolism plays an important role in the physiology of the Gl tract. It is involved in among others motility, secretion and sensitivity. ${ }^{13}$ We found that fasting serotonin levels in blood plasma of IBS patients did not differ significantly when compared to healthy controls, but interestingly, the principle metabolite of serotonin, i.e. 5-HIAA, was decreased in plasma samples of IBS patients. This was particularly pronounced in the IBS subtype characterised by a mixed stool pattern (IBS-M). Our finding may point to the role of altered serotonin metabolism in this pathophysiology of this IBS subtype, but more research is needed to investigate the association. Recently it has been shown 
that oral administration of 5-HTP, the precursor to serotonin, induced significant alterations in systemic serotonin metabolites that were accompanied by increased visceroperception of pain in hypersensitive IBS patients. ${ }^{14}$ The presence of visceral hypersensitivity is a well-known phenomenon in IBS patients. However, in what way IBS patients with normal visceral perception differ from those with visceral hypersensitivity, was unknown. In this thesis we have shown that hypersensitive IBS patients, which comprise about $50 \%$ of the total IBS population, are younger when compared to the normosensitive patients, but that other demographic characteristic, and also the use of medication, life style factors and the level of psychological symptoms do not differ between these two groups. Furthermore, the groups did not differ with respect to faecal and plasma concentrations of several biomarkers. Since the IBS patient population is very heterogeneous, it is of utmost importance to select clinically relevant subgroups which can be treated by targeted interventions. However, our findings indicate that dividing IBS patients based on visceral sensitivity may be less relevant for daily clinical practice.

The diagnosis of IBS is symptom based using the Rome III criteria, in combination with the exclusion of other organic causes if indicated. In the second part of the thesis, we investigated tools to aid the diagnostic process and reliable symptom assessment in IBS. As abdominal pain is a key-symptom of IBS we provided a systematic overview of the currently available methods to measure abdominal pain in IBS patients, but also performed a pilot study to investigate an electronic momentary symptom (ESM) assessment method, which is rather novel in the field of neurogastoenterology and motility. Repeated symptom assessment at random moments during the day using a digital tool, was found to be feasible and to provide more accurate and detailed insight on symptoms scores and fluctuations during the day. Thereby, this tools may aid in identifying triggers of symptoms in individual patients, which will be of great value to implement more personalised treatment strategies. Based on our findings, the ESM tool is currently being optimised for application in IBS and will be validated in a large international multicentre study.

Finally, this thesis focused on the quest for new biomarkers in IBS. In two separate studies we developed and validated two biomarker panels whit high sensitivity and specificity for the discrimination between IBS patients and healthy controls without GI symptoms. Both panels, a faecal and plasma biomarker panel and a panel consisting of volatile organic compounds in exhaled air, were based on a combination of markers reflecting several domains of gut health associated with the multifactorial aetiology of IBS. Both could accurately discriminate between IBS and healthy controls and furthermore did correlate with GI symptom scores. Such biomarker panels, if our results are replicated in future studies, could benefit the diagnostic process of IBS, but also provide more objective measures of treatment efficacy in intervention trials. 
In summary, the current thesis contributed to the understanding of possible aetiological mechanisms of IBS, which may be useful for more clinically relevant subtyping of IBS patients and may lead to new targets for interventions. Furthermore, we have provided an overview and evaluated new methods to assess abdominal pain in IBS patients, which may lead to more uniform (therapeutic) outcome measures in future IBS studies. This is of utmost relevance when comparing results and data between studies. We have provided first clues for a potent alternative method of symptom assessment in IBS patients. The development and validation of this tool is currently ongoing. Finally, in this thesis we identified and validated new biomarker panels for IBS patients, which may add to diagnosis and follow-up of IBS. These findings contribute to our knowledge on IBS, provide new tools for evaluating effects in therapeutic trials in IBS and help to unravel and explore potential triggers and environmental factors contributing to symptom generation.

Although at this moment a 'cure' for IBS is only an utopic future perspective, our rapidly expanding knowledge in neurogastroenterology and motility will lead to better treatment options in the near future and thereby will reduce patient discomfort and economic burden of IBS for society. 


\section{References}

1. Chey WD, Kurlander J, Eswaran S. Irritable bowel syndrome: a clinical review. JAMA. 2015;313:949-58.

2. Longstreth GF, Thompson WG, Chey WD, Houghton LA, Mearin F, Spiller RC. Functional bowel disorders. Gastroenterology. 2006;130:1480-91.

3. Canavan C, West J, Card T. The epidemiology of irritable bowel syndrome. Clin Epidemiol. 2014;6:71-80.

4. Lovell RM, Ford AC. Effect of gender on prevalence of irritable bowel syndrome in the community: systematic review and meta-analysis. Am J Gastroenterol. 2012;107:991-1000.

5. El-Serag HB, Pilgrim P, Schoenfeld P. Systemic review: Natural history of irritable bowel syndrome. Aliment Pharmacol Ther. 2004;19:861-70.

6. Simren M, Brazier J, Coremans G, Dapoigny M, Muller-Lissner SA, Pace F, et al. Quality of life and illness costs in irritable bowel syndrome. Digestion. 2004;69:254-61.

7. Gralnek IM, Hays RD, Kilbourne A, Naliboff B, Mayer EA. The impact of irritable bowel syndrome on health-related quality of life. Gastroenterology. 2000;119:654-60.

8. Canavan C, West J, Card T. Review article: the economic impact of the irritable bowel syndrome. Aliment Pharmacol Ther. 2014;40:1023-34.

9. Camilleri M. Peripheral mechanisms in irritable bowel syndrome. N Engl J Med. 2012;367:1626-35.

10. Grundmann O, Yoon SL. Irritable bowel syndrome: epidemiology, diagnosis and treatment: an update for health-care practitioners. J Gastroenterol Hepatol. 2010;25:691-9.

11. Li M, Liang P, Li Z, Wang Y, Zhang G, Gao H, et al. Fecal microbiota transplantation and bacterial consortium transplantation have comparable effects on the re-establishment of mucosal barrier function in mice with intestinal dysbiosis. Front Microbiol. 2015;6:692.

12. Karczewski J, Troost FJ, Konings I, Dekker J, Kleerebezem M, Brummer RJ, et al. Regulation of human epithelial tight junction proteins by Lactobacillus plantarum in vivo and protective effects on the epithelial barrier. Am J Physiol Gastrointest Liver Physiol. 2010;298:G851-9.

13. Gershon MD, Tack J. The serotonin signaling system: from basic understanding to drug development for functional GI disorders. Gastroenterology. 2007;132:397-414.

14. Keszthelyi D, Troost FJ, Jonkers DM, van Eijk HM, Dekker J, Buurman WA, et al. Visceral hypersensitivity in irritable bowel syndrome: evidence for involvement of serotonin metabolism--a preliminary study. Neurogastroenterol Motil. 2015;27:1127-37. 
List of publications 


\section{List of publications}

Mujagic Z / Tigchelaar EF, Zhernakova A, Ludwig T, Ramiro-Garcia J, Baranska A, Swertz MA, Masclee AAM, Wijmenga C, van Schooten FJ, Smolinska A / Jonkers DMAE. A novel gut health biomarker panel for irritable bowel syndrome and the application in the general population. Submitted

Baranska A / Mujagic Z, Smolinska A, Dallinga JW, Jonkers DMAE, Tigchelaar EF, Dekens J, Zhernakova A, Ludwig T, Masclee AAM, Wijmenga C, van Schooten FJ. Volatile Organic Compounds in breath as marker for Irritable Bowel Syndrome: a metabolomic approach. Submitted

Mujagic Z, Jonkers DMAE, Ludidi S, Keszthelyi D, Hesselink MA, Kievit RN, Althof JF, Weerts Z, Kruimel JW, Schooten FJ, Masclee AAM. Biomarkers for visceral hypersensitivity in patients with irritable bowel syndrome. Submitted

Mujagic Z, de Vos P, Boekschoten MV, Govers C, Pieters HJ, de Wit NJW, Bron PA, Masclee AAM, Troost FJ. The effects of oral administration of three Lactobacillus plantarum strains onintestinal barrier function and gene transcription in human small intestinal mucosa; a randomized double-blind placebo controlled trial. Submitted

de Vos P, Mujagic Z, de Haan BJ, Siezen RJ, Bron PA, Meijerink M, Wells J, Masclee AAM, Boekschoten MV, Faas MM, Troost FJ. Differential human mucosal transcriptomic and peripheral immune responses induced by three Lactobacillus plantarum strains; insight into how bacteria contribute to immunity in healthy individuals consuming the NSAID indomethacin. Submitted

Zhernakova A, Kurilshikov A, Bonder MJ, Tigchelaar EF, Schirmer M, Vatanen T, Mujagic Z, Vich A, Falony G, Vleira-Silva S, Wang J, Imhann F, Brandsma E, MedemaJankipersadsing S, Cenit MC, Deelen P, Weersma RK, Feskens E, Netea M, Jonkers D, Franke L, Aulchenko Y, Huttenhouwer C, Raes J, Hofker M, Xavier RJ, Wijmenga C, Fu J. Population-based metagenomics analysis reveals Chromogranin $A$ as a marker for gut microbiome composition and diversity. Submitted

Imhann F, Bonder MJ, Vila AV, Fu J, Mujagic Z, Vork L, Tigchelaar EF, Jankipersadsing SA, Cenit MC, Harmsen H, Dijkstra G, Franke L, Xavier RJ, Wijmenga C, Jonkers DMAE, Weersma RK, Zhernakova A. Proton Pump Inhibitors Affect the Gut Microbiome. Gut. Accepted; in press 
Thijssen AY / Mujagic Z, Jonkers DMAE, Ludidi S, Keszthelyi D, Hesselink MAM, Clemens $\mathrm{CM}$, Conchillo JM, Kruimel JW, Masclee AAM. Alterations in serotonin metabolism in Irritable Bowel Syndrome. Alimentary Pharmacology \& Therapeutics, 2015 Nov 5. [Epub ahead of print]

Quetglas EG, Mujagic Z, Wigge S, Keszthelyi D, Wachten S, Masclee AAM, Reinisch W. Update on pathogenesis and predictors of response of therapeutic strategies used in inflammatory bowel disease. World Journal of Gastroenterology. Accepted; in press

Tigchelaar EF, Zhernakova A, Dekens JA, Hermes G, Baranska A, Mujagic Z, Swertz MA, Muñoz AM, Deelen P, Cénit MC, Franke L, Scholtens S, Stolk RP, Wijmenga C, Feskens EJ. Cohort profile: LifeLines DEEP, a prospective, general population cohort study in the northern Netherlands: study design and baseline characteristics. BMJ Open. 2015;5: e006772.

Mujagic Z, Keszthelyi D, Aziz Q, Reinisch W, Quetglas EG, De Leonardis F, Segerdahl M, Masclee AAM. Systematic review: instruments to assess abdominal pain in irritable bowel syndrome. Aliment Pharmacol Ther 2015;42:1064-81.

Bodelier AG, Smolinska A, Baranska A, Dallinga JW, Mujagic Z, Vanhees K, van den Heuvel T, Masclee AAM, Jonkers DMAE, Pierik MJ, van Schooten FJ. Volatile Organic Compounds in Exhaled Air as Novel Marker for Disease Activity in Crohn's Disease: A Metabolomic Approach. Inflamm Bowel Dis 2015;21:1776-85.

Mujagic Z, Leue C, Vork L, Lousberg R, Jonkers DMAE, Keszthelyi D, Hesselink MA, van Schagen TJ, van Os J, Masclee AAM, Kruimel JW. The Experience Sampling Method - a new digital tool for momentary symptom assessment in IBS: an exploratory study. Neurogastroenterol Motil 2015;27:1295-302.

Mujagic Z / Ludidi S, Keszthelyi D, Hesselink MA, Kruimel JW, Lenaerts K, Hanssen NM, Conchillo JM, Jonkers DMAE, Masclee AAM. Small intestinal permeability is increased in diarrhoea predominant IBS, while alterations in gastroduodenal permeability in all IBS subtypes are largely attributable to confounders. Aliment Pharmacol Ther 2014;40:288-97.

Ludidi S / Mujagic Z, Jonkers DMAE, Keszthelyi D, Hesselink M, Kruimel J, Conchillo J, Masclee AAM. Markers for visceral hypersensitivity in patients with irritable bowel syndrome. Neurogastroenterol Motil 2014;26:1104-11. 
Straetmans J, Vent J, Lacko M, Speel EJ, Huebbers C, Semrau R, Hoebers F, Mujagic Z, Klussmann JP, Preuss SF, Kremer B. Management of neck metastases of unknown primary origin united in two European centers. Eur Arch Otorhinolaryngol 2015;272: 195-205.

\section{Patent applications}

European patent application nr. 14194725: Immunoassay for peripheral memory TCells. European patent application nr. 14194729: Immune stimulatory probiotic. Inventors: De Vos P, Troost FJ, Mujagic Z, Faas MM. Date of filing: 25.11.2014

\section{Awards and grants}

FNM 2014 Guangzhou, China, "Young Investigator Award" for the study: IBS and overreporting of abdominal pain in retrospective questionnaires: advantages of Experience Sampling Method as new digital tool in symptom measurement.

Pélerin symposium 2014, Maastricht, "Nomination for the Pélerin science award" for the study: Irritable Bowel Syndrome; from symptom to biomarkers based diagnosis.

UEGW 2014 Vienna, Austria, "Travel grant for one of best submitted abstracts" for the study: The Experience Sampling Method - a new digital tool for momentary symptom assessment in IBS.

UEGW 2014 Vienna, Austria, "Oral Free Paper Prize" for the presentation: Symptoms in patients with functional GI disorders.

DDW 2014, Chicago, USA, "Poster of distinction" for the study: Gut permeability in IBS is site-specific, subtype-dependent and affected by confounders. 
Dankwoord 


\section{Dankwoord}

Volgens sommigen is dit het belangrijkste hoofdstuk van het proefschrift. Ik weet niet of ik het daarmee eens ben, maar het is in ieder geval wel het meest gelezen deel van dit boek. Daarom wil ik ook meteen van deze gelegenheid gebruik maken. Voordat jullie het dankwoord lezen, wil ik jullie verwijzen naar hoofdstuk 1 t/m 12, alwaar jullie de laatste wetenschappelijke inzichten kunnen vinden met betrekking tot het prikkelbare darmsyndroom.

$\mathrm{Na}$ jaren werk, drie en een half jaar full time en heel veel uren naast de klinische werkzaamheden, kan ik nu eindelijk beginnen aan dit belangrijke hoofdstuk. Er zijn veel mensen die een bijdrage geleverd hebben aan mijn promotieonderzoek en mijn ontwikkeling in de afgelopen jaren. Niet iedereen kan ik hier noemen, omdat de lijst te lang zou worden, maar een aantal wil ik in dit dankwoord toch nadrukkelijk bedanken.

Allereerst, de persoon die het meeste 'te lijden' heeft gehad onder mijn volharding om toch zo veel mogelijk uit mijn promotietijd te halen. En tegelijk de persoon die mij het meeste hierin gesteund heeft.

Lieve Eveline, graag wil ik je bedanken voor het regelen van het promotiefeest en het drukwerk. Maar ik wil je met name bedanken voor je begrip in de afgelopen jaren. Je accepteerde mijn lange werkdagen en weekenden achter de laptop en je steunde me in al mijn nieuwe ideeën. Op de een of andere manier zorgde je er altijd voor dat het werkte, dat we altijd tijd vonden voor elkaar en dat ik energie had om weer een nieuwe studie te starten. Door jou kon ik er zeker van zijn dat hoe stressvol een dag ook was ik altijd thuis een glimlach op mijn gezicht kreeg als ik je zag. In het laatste jaar van mijn promotietijd heb je er dan ook nog (mede) voor gezorgd dat ik thuis niet een, maar zelfs twee prachtige dames kon verwachten na de werkdag. En ondanks dat kleine Amila het dagelijks leven een stuk drukker maakte, heeft ze me zoveel meer levensvreugde en geluk gebracht.

Draga majka, dragi tata, zonder jullie was dit nooit gelukt! Jullie opvoeding heeft ervoor gezorgd dat het belang van kennis, opleiding, inzet en een positieve instelling in het leven aan mij al vanaf de jonge jaren duidelijk was. Jullie hebben mij altijd gesteund en jullie hebben altijd laten blijken hoe trots jullie zijn. Ik weet dat jullie heel wat in jullie leven hebben moeten opgeven om ervoor te zorgen dat ik een opleiding aan de universiteit kon volgen en daar blijf ik jullie altijd dankbaar voor!

Het was zwaar voor jullie om met vier kinderen, waarvan twee nog zeer jong, na jaren van oorlog, een nieuw leven te moeten opbouwen. En dat zonder eigen bezittingen, zonder onderdak of werk in een land met een andere cultuur en een andere taal. En toch is het jullie, met wat hulp van goede mensen, gelukt om snel jullie draai te vinden en een nieuw bestaan te realiseren. Jullie hebben ervoor gezorgd dat wij nooit iets te 
kort kwamen en dat er een toekomst was waar we naar toe konden werken. Nu alle vier de kinderen volwassen zijn, hun eigen leven leiden en hard werken aan hun carrière, kunnen jullie van het welverdiende pensioen genieten. Alhoewel, er zijn natuurlijk tegenwoordig wel enkele kleinkinderen waar jullie soms je handen aan vol hebben.

Dragi Satko i Nacko, uiteraard horen jullie namen bij de belangrijkste personen die in dit dankwoord voor moeten komen. Ik heb altijd twee grote broers gehad naar wie ik op kon kijken. Mijn twee grote idolen die hun puberjaren zijn kwijtgeraakt door de moeilijke jaren '90, maar desondanks zo veel wisten te bereiken in hun leven. Jullie hebben me laten zien dat hard werken loont. Daarnaast heb ik uiteraard jullie 'regelmatige financiële hulpverlening' ook zeer gewaardeerd. En daarnaast heb ik aan jullie ook drie geweldige nichtjes, Anisa, Lejla en Mila, te danken en daarmee ook de belangrijke titel 'striko'.

Draga Zlata, wij zijn altijd een team geweest. Ook al denken veel mensen dat ik voornamelijk op jou gepast heb, denk ik dat jij veel meer voor mij hebt betekend. Onze ouders hebben ons niet voor niets een gelijkklinkende naam gegeven. Mijn kleine zusje, die altijd voor me klaar stond, die er altijd was, en die destijds als 12-jarige in grote spanning mijn toelatingsbrief tot de Universiteit van Maastricht afwachtte, omdat ze bang was dat ik eventueel ver weg zou gaan studeren. En toen, toen de brief eindelijk kwam, en jij even alleen thuis was: de brief opende, maar niet begreep wat er in de brief stond en of je grote broer nou in Maastricht mocht studeren of niet. Tegenwoordig werken we zelfs samen in het azM, en geniet ik regelmatig van onze gesprekken carpoolend naar het werk. Ik ben blij dat ik zo een lief zusje heb.

Lieve Marleen, Lou en jij hebben mede mijn leven bepaald in het afgelopen decennium. Graag had ik ook Lou erbij gehad tijdens mijn promotie. Ik weet hoeveel plezier hij haalde uit het feit dat Eveline, maar ook ik, het zo goed deden op de universiteit. En net als jij, heeft hij zijn trots vaak laten blijken. We zijn gegroeid tot een hechte familie en je steun voor Eveline en mij in de afgelopen jaren waardeer ik zeer. Je bent een lieve oma voor Amila en je helpt ons graag. Bij dezen wil ik je dan ook ontzettend bedanken voor alles wat je gedaan hebt.

En dan natuurlijk het MDL-onderzoeksteam. Stuk voor stuk harde werkers, maar met name fantastische collega's. Als eerste begin ik met mijn paranimfen, Tim en Mark.

Tim, ik heb toch enkele jaren een kantoor met je mogen delen. En ondanks je 'zwakke voetbalkwaliteiten' heb ik veel plezier gehad op UNS 40, niveau 5, ruimte 5.567. Ik schrijf dit bewust zo specifiek op, omdat ik weet dat je zelfs na al die jaren je exacte locatie in het universiteitsgebouw niet kan noemen. Ik heb je leren waarderen als onderzoeker, als collega en vriend, maar ook als filosoof. Tussen de wetenschappelijke 
discussies door heb ik altijd genoten van onze gesprekken over de politiek, de maatschappij, maar ook uiteraard sport.

Mark, we kennen elkaar al vanaf de eerste onderwijsgroep in de zomer van 2004. En toen al konden we goed opschieten. In 2011 heb ik gesolliciteerd bij MDL in Maastricht en kon ik er uiteraard op vertrouwen dat jij een goed woordje voor me deed, alvorens ik bij prof. Masclee, Yolande Keulemans en later bij Freddy op sollicitatiegesprek mocht verschijnen. We hebben tijdens onze gezamenlijke promotietijd veel leuke momenten meegemaakt, van de clubs in Florida tot uiteraard de onvergetelijke Club Moustacheparty's.

Heren, ik ben blij dat jullie het paranimfschap hebben aanvaard!

Daarnaast zijn er nog een paar collega's die ik hier uitdrukkelijk wil noemen.

Allereerst Samefko. Als IBS-duo hebben we samen een belangrijke bijdrage geleverd aan de opbouw van het Maastricht IBS cohort, en hebben we samen met andere collega's een mooie basis gelegd voor verder wetenschappelijk onderzoek in Maastricht op dit gebied. Ik herinner me nog een van de eerste dagen op het werk, met al een berg opdrachten en mijn minimale wetenschappelijke kennis. Je kwam op mijn kantoor en zei: "We zijn hier een team, dus probeer vooral niet het wiel zelf uit te vinden. Je mag me storen met vragen wanneer je maar wilt." En je hebt je aan je woord gehouden. Van het uitvoeren van de barostat tot de Mann-Whitney U-test, ik heb veel van je mogen leren. En bovenal, ik heb met veel plezier met je samengewerkt. Bedankt!

Dan mijn twee andere roomies. Steven, ik ken je nog als coassistent in Roermond, waar ik als zaalarts werkte op de afdeling. Jij was de gedreven student die toen al wist dat hij MDL-arts ging worden. Later, bij MDL in Maastricht, werden we kantoorgenoten. Met jou en Tim op de kamer heb ik een geweldige onderzoekstijd gehad. Het was altijd gezellig en we vonden zelfs tijd om een 'betaald voetbalcompetitie' op te zetten, maar dat betekende niet dat er niet hard gewerkt werd. Hoe vaak hebben we niet samen naar een bepaalde zin in een artikel van een van ons getuurd en deze tien keer veranderd, totdat we het erover eens waren dat het zo goed was. Bedankt voor je gezelligheid en je collegialiteit!

Elhaseen, de rustige man uit Soedan, die geweldige humor bleek te hebben. Met een mix van Engels en Nederlands wist je altijd op de meest onverwachte momenten de beste opmerkingen te maken. Graag wil ik je ook bedanken voor je hulp bij laboratoriumwerkzaamheden. Je uitgebreide kennis op dit gebied was onmisbaar in het eerste jaar van mijn promotie.

Dan Daniel, de andere collega met de moeilijke achternaam. Al vanaf het begin kon ik je goedbedoelde 'gemopper' waarderen. Je bent collegiaal en ik heb zeer veel van je geleerd. Je onuitputtelijke kennis over de laatste publicaties kwam meer dan eens goed van pas. Aan verschillende artikelen in dit proefschrift heb je een belangrijke bijdrage geleverd en ik ben je daar zeer dankbaar voor. Al jaren werk je er hard aan om Maastricht op de neurogastro-enterologische wereldkaart te zetten en je boekt goede 
vooruitgang. Van onze autotour naar Nantes naar het lab van Michel Neunlist, heb ik genoten. Helaas is er geen wetenschappelijk vervolg op gekomen, maar wie weet. $\mathrm{Na}$ de promotie heb ik toch weer wat tijd te vullen.

Fedde, onze one-and-only mister Nicolaas, ik heb graag met je samengewerkt en het blijft toch jammer dat je naar het noorden bent getrokken. Ik heb altijd graag met je gediscussieerd over zaken als mastcelstabilisatoren, gevoetbald en gefeest. Met o.a. Maarten en dr. G. - Givan hebben we een goede tijd in Orlando gehad. Een nieuwe reünie gaat er zeker komen.

Uiteraard mag ik de feestbeesten van de MDL niet vergeten. Chanti (Chantal) en Furby (Bouke), jullie wisten van iedere gelegenheid een goed feest te maken. Maar ook op de werkvloer hebben jullie verhalen de werkdagen een stuk leuker gemaakt. Volgens mij was de toevoeging van Roel, met zijn geweldige droge opmerkingen, aan jullie kantoor ook een goede aanvulling hierop.

Dan uiteraard Freddy. Ik ben je zeker dank verschuldigd voor alle hulp en begeleiding tijdens mijn eerste jaar als onderzoeker in Maastricht, maar ook voor de gezellige gesprekken en de borrels en barbecues in de jaren daarna.

Martine, graag wil ik jou bedanken voor al het geduld en werk dat je steekt in de opbouw van het MIBS cohort, je hulp met de data, maar ook voor je vaak relativerende woorden op allerlei 'grote' problemen waar ik door de jaren tegenaan liep.

Helaas kan ik niet over iedereen een lang verhaal schrijven, want dan wordt het wel een heel lang dankwoord, maar Marin, Bas, Kirsten Pijls, Kirsten van der Beek, Ellen, Pauline, Annick, Roy, Anke, Selwyn, Dirk, Irene, Harm-Jan, Esther, Mariëlle, Eveline, Montserrat, Fabienne, Sander, Danyta en uiteraard het droomduo Lisa en Zsa Zsa, graag wil ik jullie bedanken voor de samenwerking, de leuke lunchgesprekken, de werkfeesten en jullie hulp in de afgelopen jaren. Corinne, tegenwoordig de fantastische collega op B5, wil ik natuurlijk graag apart bedanken voor de gezelligheid tijdens mijn onderzoekstijd. We moeten trouwens nog steeds samen het IBS-IBD-FFQ-artikel schrijven.

Tevens ben ik dank verschuldigd aan de UM onderzoeksschool NUTRIM, en met name aan prof. Annemie Schols en Sef Janssen, voor het raamwerk waarin ik mijn promotieonderzoek heb mogen uitvoeren.

Daarnaast wil ik ook alle clinici bedanken die hun steen bij hebben gedragen aan de patiëntinclusies en de studie-endoscopieën. Allereerst de MDL-artsen, naast prof. Masclee ook José Conchillo, Rogier De Ridder, Wim Hameeteman, Ger Koek, Silvia Sanduleanu, Marieke Pierik, Yolande Keulemans en Joanna Kruimel, bedankt voor jullie begeleiding en hulp. Uiteraard wil ik hier ook de arts-assistenten (van toen) noemen: Jeoffrey, Michel, Chantal, Frank, Jos, Paul, Bram, Reggy, Linda, Tamara, Gerben en Maarten. Annemieke wil ik apart bedanken, mede voor de goede samenwerking op o.a. het serotonine artikel, met uiteindelijk een mooi resultaat. 
Natuurlijk niet te vergeten, Ton Mestrom en zijn endoscopieteam, de collega's van de functiekamer, Pierre, Ria en Nicole, maar ook Yvan, Henny, Mia en Andrea. En uiteraard Mietsie en Elly. Altijd een glimlach en altijd een oplossing voor al mijn kleine problemen. Tot slot wil ik ook heel graag Tiny bedanken voor alle logistieke hulp gedurende mijn onderzoekjaren, maar met name voor de hulp bij de lay-out van dit proefschrift!

ledereen binnen de MDL-afdeling, hartelijk bedankt voor jullie steun!

Graag wil ik ook alle collega's van TiFN bedanken. Het is een fantastische organisatie die bijzondere kansen biedt aan jonge onderzoekers. Ik heb via TiFN met veel interessante mensen mogen samenwerken en sommigen wil ik hier nog nadrukkelijk noemen. Met name wil ik het team van project GH001 bedanken: naast Daisy en Frederik-Jan, ook Jackie (bedankt voor je enthousiasme), Cisca (bedankt voor je leiding), Ettje en Sasha (bedankt voor de goede samenwerking), maar ook Gerben, Edith, Hauke, Jan, Javier, Erwin, Mark-Jan en Morris. Agi (Agnieszka Smolinska) en Agnieszka Baranska wil ik apart noemen. Dames, bedankt voor de goede samenwerking en collegialiteit, de statistische lessen en de gezelligheid in de trein naar de TiFN meetings. Ondanks, maar waarschijnlijk juist dankzij, de grote verscheidenheid in achtergronden van de leden van het TiFN-projectteam en daardoor vaak verschillen in opvattingen, is dit een topteam geweest. Het project heeft wetenschappelijk reeds veel bereikt en ik denk dat menig onderzoeker de komende jaren de vruchten kan plukken van ons werk binnen GH001. Ik ben blij dat ik hier deel van mocht uitmaken! And of course I would like to thank the experts. Arjen, Thomas, Gabriela, Annick, Clara, Thomas, Anja, Joseph and Kathy, thank you for your input and support!

Tevens wil ik ook Marjolein, Jerry, Coen, Nicole, Mark, Peter en Roger bedanken voor de hulp bij de probiotica-studies. En uiteraard Paul, ondertussen prof. De Vos, bedankt voor je vertrouwen in mij en voor je hulp. Ik ken niemand die sneller zijn mails beantwoord, maar ook altijd inhoudelijk een goed antwoord heeft. Daarnaast wil ik je ook bedanken voor de gezellige diner- en baravonden bij TiFN.

Joanna Kruimel en Carsten Leue wil ik graag bedanken voor de hulp bij de ESM-studie, maar ook met name voor alle leermomenten over de psychosomatiek, het onderwerp dat zo belangrijk is binnen de functionele maagdarmaandoeningen. Ik vond het een zeer prettige samenwerking. Ik vind het jammer dat ik de MPU-trial niet heb kunnen afronden samen met jullie, maar ik weet zeker dat de komende jaren daar nog prachtige data uit gaan rollen. In dit kader bedank ik ook prof. Jim van Os, Richel Lousberg, maar ook Truda Driessen voor de praktische, statistische en inhoudelijke bijdrage en hulp bij het ESM-project.

Ook prof. Jean Muris ben ik dank verschuldigd voor de vruchtbare samenwerking met de huisartsen in regio Maastricht. 
I would like to thank the 'non-Maastricht members of abdominal pain team': among others Emilio Quetglas, Francesco De Leonardis, Märta Segerdahl, prof. Qasim Aziz and Walter Reinisch.

Bij de MDL ben ik terecht gekomen mede door het enthousiasme van de MDL-artsen in het Laurentius ziekenhuis te Roermond. Daarom wil ik graag Paul Bus, Eric de Goede en Guido Houtmeyers bedanken, maar bovenal ben ik in dit kader dank verschuldigd aan Susan Toebosch voor haar vertrouwen, steun, de vele leermomenten en de liefde voor het vak die ze goed weet over te brengen.

Tevens wil ik ook prof. Bernd Kremer, Ernst-Jan Speel en Jos Straetmans bedanken voor de begeleiding tijdens mijn wetenschapsstage in 2010 en mijn eerste leermomenten binnen de medische wetenschap.

Gedurende mijn onderzoeksjaren heb ik met veel plezier veel studenten mogen begeleiden, die tevens een bijdrage hebben geleverd aan dit proefschrift. Caroline Botterweck, Charlotte Bruijsten, Esther Jansen, Hanan Rachid, Jeroen van den Akker, Jory Molema, Ruud Kiviet, Ruud Verhees, Vico Leeters, Tiny Castermans, Vicky Weel, Reman Shaker, Thirza van Schagen, Wesam Moafa, Michaela Höhn, Zoë van Lierop, Jessica Althof en uiteraard de tegenwoordig gewaardeerde collega's Zsa Zsa Weerts en Lisa Vork, allen bedankt voor jullie hulp!

In de verschillende studies in dit proefschrift heb ik data van honderden proefpersonen verwerkt, zowel van PDS-patiënten als mensen zonder gezondheidsklachten. Zonder de bereidwillendheid van al deze mensen om een bijdrage te leveren aan de medische wetenschap was ook dit proefschrift er nooit geweest. Daarom ben ik met name jullie enorme dank verschuldigd voor jullie deelname.

De leden van de beoordelingscommissie, te weten prof. dr. Dejong (voorzitter), prof. dr. Schols, prof. dr. Spiller, prof. dr. Steinbusch en dr. Van der Veek, wil ik graag bedanken voor de evaluatie van dit proefschrift.

Aan het einde van dit dankwoord wil ik diegenen bedanken die het meest bijgedragen hebben aan dit proefschrift. Ik had de eer om mijn promotieonderzoek uit te voeren onder supervisie van mijn copromotor dr. Daisy Jonkers en mijn promotoren prof. dr. Frederik-Jan van Schooten en prof. dr. Ad Masclee.

Beste Daisy, ik had me geen betere copromotor kunnen voorstellen. Zonder jouw begeleiding en input had ik dit proefschrift nooit kunnen schrijven. Je hebt een brede kennis, je methodologisch inzicht is geweldig, maar bovenal ben je een kei in het begeleiden van jonge onderzoekers. Je straalt vertrouwen uit, en je hebt je best gedaan om me te helpen bij alle kleine en grote wetenschappelijke, praktische en 
privéproblemen die gedurende mijn promotietijd ontstonden. Waar ik me nog steeds over verbaas, is je gave om kritiek als een compliment te brengen. Zelfs je 'track changes' in Microsoft Word zijn blauw i.p.v. rood, zodat het aardiger overkomt. Ik ben blij dat je mijn supervisor bent geweest! Bedankt voor alles!

Dan prof. Van Schooten, beste Frederik-Jan, als tweede promotor bent $u$ iets minder nadrukkelijk betrokken geweest bij mijn alledaagse werkzaamheden. Desondanks hebt $u$ een belangrijke bijdrage geleverd aan dit proefschrift. Uw wetenschappelijke en organisatorische kwaliteiten kwamen regelmatig van pas en ik heb veel van u mogen leren. Daarnaast was u altijd een gezellige gesprekspartner, ook als het niet over de wetenschap ging. Bedankt voor al uw hulp en de goede begeleiding!

En uiteraard als laatste in dit hoofdstuk; prof. Masclee, beste Ad, toen ik in 2011 aan tafel in uw kantoor zat tijdens mijn sollicitatiegesprek wist ik niet dat ik het komende decennium onder uw leiding zou gaan werken. Die dag dacht ik dat, als ik aangenomen wordt, het wel pittige jaren zouden worden met zo een strenge baas. Dat was tenminste de eerste indruk. Het tegendeel is waar. $U$ bent betrokken, eerlijk en duidelijk. $U$ stond altijd voor me klaar als het nodig was. Het was geen uitzondering dat ik een deadline moest halen voor een abstract of een artikel en dat ik uw commentaren om half 8 op zondagochtend via mail binnenkreeg. Ik heb u ontzettend leren waarderen als supervisor. Uw gedrevenheid en liefde voor het medische vak en de wetenschap is inspirerend. Uw expertise binnen de neurogastro-enterologie en motiliteit heeft dit proefschrift gevormd. Graag wil ik u bij dezen dan ook bedanken voor alle hulp, de goede begeleiding en steun in de afgelopen jaren. Ik verheug me op de klinische werkzaamheden onder uw supervisie de komende jaren. 
Curriculum vitae 


\section{Curriculum vitae}

Zlatan Mujagić was born on the 26th of June 1985 in Prijedor, Bosnia and Herzegovina. In October 1994 he emigrated to the Netherlands, where he finished primary and secondary school in the municipality of Stein. In 2004, he started his medical education at Maastricht University. He has received his bachelor and master degree in 2007 and 2010, respectively. During this study period he spent one semester at the Otorhinolaryngology clinic of the University Clinical Centre Sarajevo. He completed his scientific internship at the department of Otorhinolaryngology at the University hospital of Maastricht and the department of Molecular Cell Biology at the at Maastricht University, studying the relation between the human papilloma virus and head and neck cancer of unknown primary origin. After obtaining his medical degree, he started working at the departments of Internal Medicine and GastroenterologyHepatology at the Laurentius hospital Roermond. One year later he started as PhDfellow at the division Gastroenterology-Hepatology, department of Internal Medicine, Maastricht University Medical Center+ (MUMC+), under the supervision of Dr. D. Jonkers, Prof. dr. FJ van Schooten and Prof. dr. A. Masclee. The research was performed within NUTRIM School of Nutrition and Translational Research in Metabolism (Maastricht University) and in part funded by the Top Institute for Food and Nutrition (TIFN), Wageningen. At the $1^{\text {st }}$ of December 2014, he started his residency Gastroenterology-Hepatology under supervision of Prof. dr. A. Masclee and is currently working at the department of Internal Medicine at the MUMC+ under supervision of Prof. dr. C. Stehouwer. Zlatan is married to Eveline and they live together with their daughter Amila. 
